\title{
FACTORS ASSOCIATED WITH ADULT PERIODONTITIS IN A DENTAL TEACHING-CLINIC POPULATION
}

\author{
BY \\ SHIRLEY CLAIRE GELSKEY \\ BSc (Marquette), MPH (Michigan, Ann Arbor)
}

\begin{abstract}
A Thesis
Submitted to the Faculty of Graduate Studies in Partial Fulfillment of the Requirement for the Degree of
\end{abstract}

Doctor of Philosophy

Department of Community Health Sciences

Faculty of Medicine

University of Manitoba

Winnipeg, Manitoba

September 28, 1995 
The author has granted an irrevocable non-exclusive licence allowing the National Library of Canada to reproduce, loan, distribute or sell copies of his/her thesis by any means and in any form or format, making this thesis available to interested persons.
L'auteur a accordé une licence irrévocable et non exclusive permettant à la Bibliothèque nationale du Canada de reproduire, prêter, distribuer ou vendre des copies de sa thèse de quelque manière et sous quelque forme que ce soit pour mettre des exemplaires de cette thèse à la disposition des personnes intéressées.

L'auteur conserve la propriété du droit d'auteur qui protège sa thèse. Ni la thèse ni des extraits substantiels de celle-ci ne doivent être imprimés ou autrement reproduits sans son autorisation. 
Name

Dissentation Abstracts International is arranged by broad, general subject categories. Please select the one subject which most nearly describes the content of your dissertation. Enter the corresponding four-digit code in the spaces provided.

Public Health SUBECT IERM
0.51773 U.M.I SUBJECT CODE

\section{Subject Categories}

\section{THI HUMANITIES AND SOClal SCIENCES}

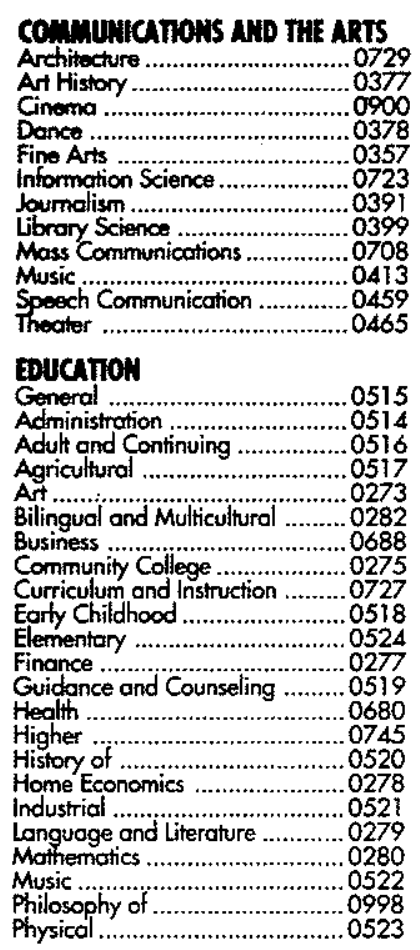

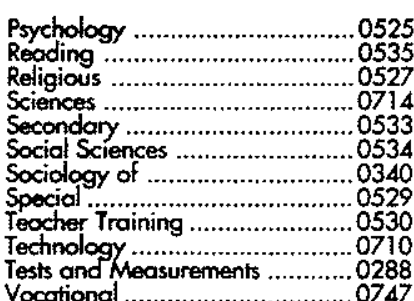

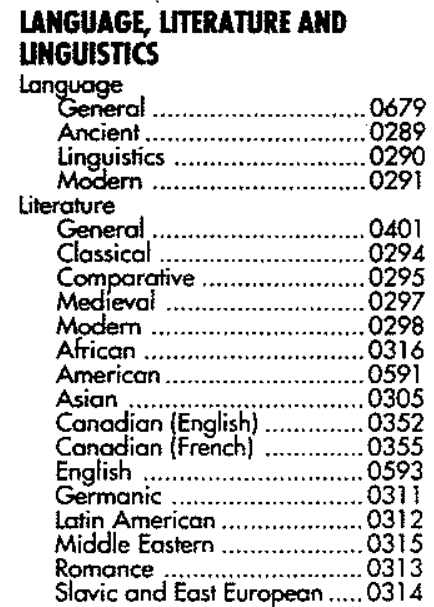

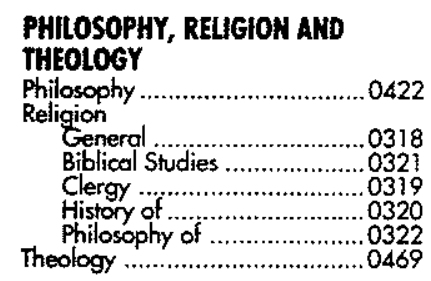

\section{SOCIAL SCENCES}

American Studies .......................0323

Anthropology

Archaeology ……............... 0324

Cultural …….......................0326

Business Administration

General .............................0310

Accounting …….................... 0272

Bonking ............................0770

Morketing …….....................0338

Conadian Studies .........................0385

Economics

General ................................ 0501

Agricultural .........................0503

Commerce-Business ...................0505

Finonce ……........................

History

Labor

Theory .

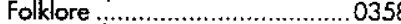

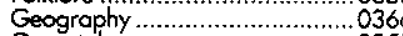

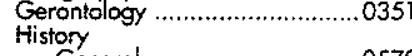

.0578

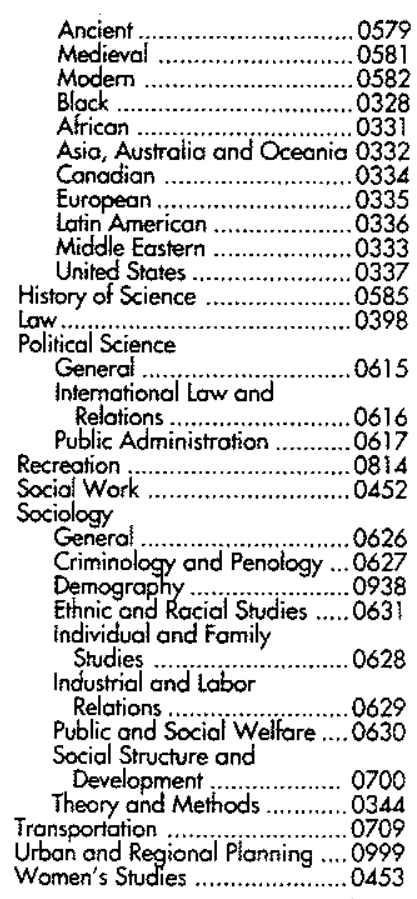

THE SCIENCES AND ENCINEERINO

\section{BIOLOGICAL SCIENCES}

Agriculture,

General ..............................0473

Anronomy l...........

Nutrition and 0475

Animal Pothology ...................0476

Food Science and

Technology ......................0359

Forestry and wild life .............0478

Plant Culture …………….....0479

Plant Physiology .....................0817

Range Management ..............0777

Wood Technology .................0746

Biology

General ...............................0306

Anatomy …………............028

0308

0379

Ecology

Entomolagy ……………....... 0353

Enfomology ……................. 0369

Genetics ...........................0369

Microbiology ........................... 0410

Molecular .............................0307

Neuroscience .......................0317

Oceanography .....................0416

Physiology …………….......0433

Radiation .........................0821

Rediation …………….......0878

Veterinary Science ...............0778

Biophysics

General ...........................0786

EARTH SCIENCES

Biogeochemistry
Geochemistry.

0425

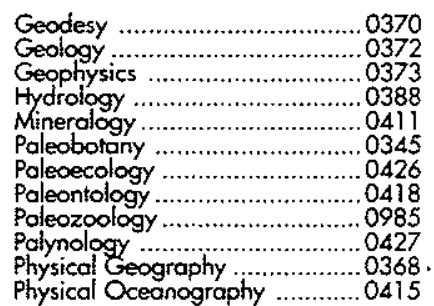

\section{HEALTH AND ENVIRONMEMTAL}

\section{SCIENCES}

Environmental Sciences .............0768

Health Sciences

Generd ............................. 0566

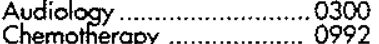

Chemotherapy …................. 0992 .

Education ………......................0350

Hospital Management ..........0769

Human Development ............078

Medicine and Surgery ...........0564

Medicin Health Surgery ……...0347

Mental Health .....................0347

Nursing ............................0569

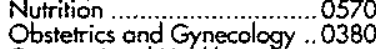

Occupational Health and

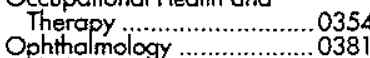

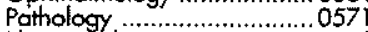

Pharmacology ......................0419

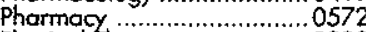

Physical therapy ...................0 0382

Public Health ......................... 0573 . 0574
Rodiology .......................

Rediology ……...................0574
Speech Pothology ................0460 Home Economics .............................0386

\section{PHYSICAL SCIENCES}

\section{Pure Sciences}

Chemistry

General .... 0485

Agricultural ……..................... 0749

Andytical .......................................

Biochemistry ...........................0487

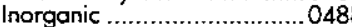

Nuclear ….................................0738

Orgonic ...............................0490

Pharmaceutical ....................049 049

Polymer ....................................0495

Radiction ……….............................. 075

Mathemotics ..................................... 0405

Physics

General ..............................0605

Acoustics ..............................0986

Astronomy and

Atmospheric Science.................0608

Atmospheric Science ............0608

Atomic ........................0748
Electronics and Electricity ....0607

Elementary Particles and

High Energy......................0798
Fluid and Plasma...

Molecular ...............075

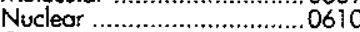

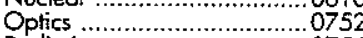

Radiation ............................0756

Solid State ............................0611

Applied Sciences

Applied Mechanics ....................0346

Computer Science ........................0984

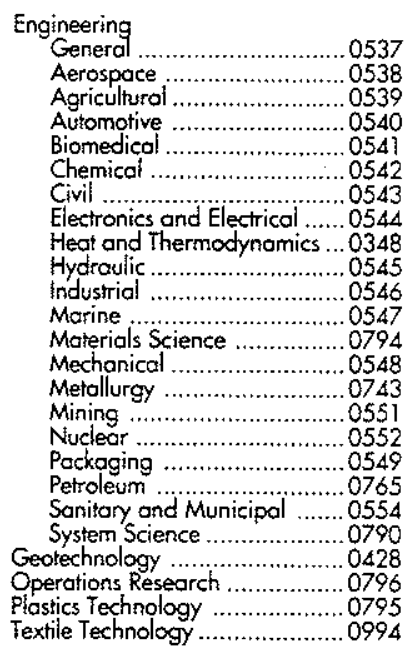

\section{PSYCHOLOGY}

General

Behavioral ......................................... 038

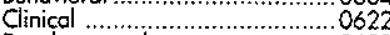

Developmental ..........................0620

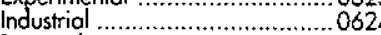

Persondlity .......................................0625

Psychobiology ………………....... 0349

Psychometrics …………………....0632

Social ........................................... 045 82 28 32 34 333 $\sqrt{3}$ 8 814
452 26 38

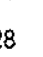
00 4 .

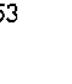


FACTORS ASSOCIATED WITH ADULT

PERIODONTITIS IN A DENTAL

TEACHING-CLINIC POPULATION

BY

SHIRLEY CLAIRE GELSKEY

A Thesis submitted to the Faculty of Graduate Studies of the University of Manitoba in partial fulfillment of the requirements of the degree of

DOCTOR OF PHILOSOPHY

(c) 1995

Permission has been granted to the LIBRARY OF THE UNIVERSITY OF MANITOBA to lend or sell copies of this thesis, to the NATIONAL LIBRARY OF CANADA to microfilm this thesis and to lend or sell copies of the film, and LIBRARY MICROFILMS to publish an abstract of this thesis.

The author reserves other publication rights, and neither the thesis nor extensive extracts from it may be printed or other-wise reproduced without the author's written permission. 
TABLE OF CONTENTS

$\mathrm{i}-\mathrm{iv}$

ABSTRACT

$\mathrm{v}-\mathrm{vi}$

LIST OF TABLES

vii - ix

ACKNOWLEDGEMENTS

$\mathrm{x}$

\section{PART I \\ INTRODUCTION AND BACKGROUND}

CHAPTER ONE - Introduction

1.1 Research Goals 1

1.2 Statement of The Problem 1

CHAPTER TWO - Epidemiology of Adult Periodontitis 3

2.1 Definition 3

2.2 Prevalence and Measurement Issues 3

2.3 Severity, Extent and Distribution 6

2.4 Natural History $\quad 7$

2.4.1 Disease Initiation $\quad 7$

2.4.2 Disease Progression 8

2.5 Public Health Impact 9

2.5.1 Economic Costs and Lost Productivity 10

2.5.2 Influences on Quality of Life 11

$\begin{array}{lll}2.6 & \text { Conclusions } & 11\end{array}$

CHAPTER THREE — Risk Assessment 13

$\begin{array}{lll}3.1 & \text { Conceptual Issues } & 13\end{array}$

3.1.1 Relative Measures of Disease Occurrence 13

3.1.2 Risk Factors, Markers, Indicators and Determinants 13

3.1.3 Cause versus Association 14

3.1.4 Risk Assessment 15

3.1.5 Models and Criteria for Causation 16

3.1.6 Conclusions 18 
$\begin{array}{lll}4.0 & \text { Theoretical Framework } & 19\end{array}$

$\begin{array}{lll}4.1 & \text { Sociodemographics } & 20\end{array}$

4.1.1 Age $\quad 20$

4.1.2 Gender 24

4.1.3 Income, Education, Race 25

4.2 Health Compromising/ Behavior $\quad 27$

$\begin{array}{ll}\text { 4.2.1 Cigarette Smoking } & 27\end{array}$

4.3 Chronic Conditions/Diseases 33

4.3.1 Diabetes 33

4.3.2 Hypertension/Ischemic Heart Disease 38

4.4 Medications with Potential Drug Action 43

$\begin{array}{lll}4.5 & \text { Oral Finding } & 46\end{array}$

4.5.1 Plaque 46

4.6 Conclusions $\quad 55$

\section{PART II \\ METHODOLOGY}

CHAPTER FIVE — Design Options For Risk Assessment 56

$\begin{array}{lll}5.0 & \text { Introduction } & 56\end{array}$

$5.1 \quad$ Experimental versus Observational Studies 56

$\begin{array}{lll}5.2 & \text { Analytic Designs } & 57\end{array}$

5.2.1 Cross-Sectional Studies $\quad 57$

5.2.2 Cohort Studies 58

5.2.3 Case-Control Studies $\quad 60$

$\begin{array}{lll}5.3 & \text { Study Bias } & 61\end{array}$

5.4 Conclusions 66 
CHAPTER SIX — Study Design 68

$\begin{array}{lll}6.0 & \text { Introduction } & 68\end{array}$

6.1 Study Setting and Target Population 68

6.1.1 Inclusion Criteria $\quad 69$

$\begin{array}{ll}\text { 6.1.2 Exclusion Criteria } & 69\end{array}$

6.2 Sample Size and Study Power 71

6.3 Sampling Frame $\quad 72$

6.4 The Dependent Variable $\quad 72$

6.4.1 Case Definition $\quad 74$

6.4.2 Control Definition 74

6.5 Independent Variables: Methods of Data Collection 75

$\begin{array}{lll}\text { 6.5.1 Chart Review } & 75\end{array}$

6.5.2 Radiographic Analysis $\quad 76$

$\begin{array}{ll}\text { 6.5.3 Telephone Interview } & 80\end{array}$

6.6 Concordance of Diagnostic Criteria $\quad 83$

6.6.1 Differences Between Cases and Controls: 85 Six Diagnostic Criteria

6.7 Ethical and Access Considerations $\quad 85$

6.8 Study Limitations 86

CHAPTER SEVEN - Validity and Reliability of Measurements 89

$\begin{array}{lll}7.1 & \text { Validity and Reliability } & 89\end{array}$

$\begin{array}{lll}\text { 7.1.1 Types of Validity } & 89\end{array}$

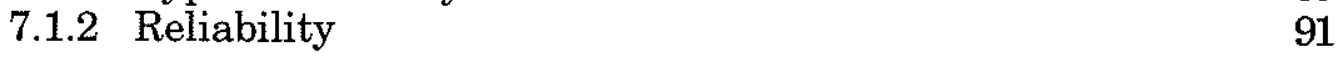

7.2 Secondary Clinical Documents $\quad 91$

7.2.1 Clinical Attachment Loss/Level 92

7.2.2 Radiographic Evidence of Bone Loss 94

$\begin{array}{ll}\text { 7.2.3 Probing Depth } & 102\end{array}$

$\begin{array}{ll}7.2 .4 & \text { Plaque Scores } \\ 7.2 .5 & 105\end{array}$

$\begin{array}{ll}\text { 7.2.5 Bleeding On Probing } & 108\end{array}$ 


\section{PART III \\ RESULTS AND CONCLUSIONS}

CHAPTER EIGHT - Analysis and Results

$\begin{array}{lll}8.1 & \text { Objectives of Analysis } & 116\end{array}$

$\begin{array}{lll}8.2 & \text { Data Entry } & 117\end{array}$

$\begin{array}{lll}8.3 & \text { Screening of Data } & 117\end{array}$

$\begin{array}{lll}\text { 8.4 The Study Population } & 117\end{array}$

$\begin{array}{lll}\text { 8.5 Participants versus Non-Participants } & 118\end{array}$

$\begin{array}{lll}8.6 & \text { Reliability, Validity, and Quality of Data } & 120\end{array}$

$\begin{array}{lll}8.7 & \text { Univariate and Bivariate Analyses } & 126\end{array}$

$\begin{array}{lll}8.8 & \text { Multivariate Analyses } & 147\end{array}$

$\begin{array}{ll}\text { 8.8.1 Preliminaries to Model Building } & 147\end{array}$

CHAPTER NINE - Discussion and Conclusions 165

$\begin{array}{lll}9.0 & \text { Overall Findings } & 165\end{array}$

9.1 Explanatory Variables 166

9.1.1 Sociodemographic Factors 166

9.1.2 Socioeconomic Status Variables 171

9.1.3 Health Compromising Behavior 176

9.1.4 Chronic Conditions/Diseases 183

9.1.5 Use of Medication with Potential Drug Actions 186

9.1.6 Oral Findings 187

$\begin{array}{lll}9.2 & \text { Conclusions } & 190\end{array}$

$\begin{array}{lll}9.3 & \text { Recommendations } & 192\end{array}$

$\begin{array}{lll}9.4 & \text { Implications } & 193\end{array}$

$\begin{array}{ll}\text { REFERENCES } & 205\end{array}$

APPENDICES 224 


\section{FACTORS ASSOCIATED WITH ADULT PERIODONTITIS IN A DENTAL TEACHING-CLINIC POPULATION}

\section{ABSTRACT}

\section{BACKGROUND:}

Periodontitis is a leading cause of tooth loss among adults. The prevalence of moderate-severe periodontitis in North America is 30\% and $10 \%$ respectively. Studies have attempted to identify individuals at increased risk of developing periodontitis. Several risk factors, indicators and determinants have been reported.

\section{GOALS:}

The goals of the research were:

1. To determine antecedent exposure of individuals with periodontitis to specific health-compromising behaviors, medications, chronic diseases / conditions, socioeconomic status, and oral findings.

2. To assess the presence and strength of association of these exposures with a history of periodontitis.

\section{METHODS:}

A case control study was carried out using secondary clinical and radiographic data from existing records obtained from the teaching-clinic population, Faculty of Dentistry, University of Manitoba. Subjects ( $n=410$ ), aged $\geq 35$ years were identified as case or control. Exposure to antecedent explanatory variables was assessed through blind telephone interviews with a response rate of $93.4 \%$.

\section{ANALYSES AND RESULTS:}

Univariate and bivariate analyses revealed significant relationships between a history of periodontitis and several explanatory variables. Analyses were age and gender-adjusted and the population age-stratified, ( $35-54$ and $\geq 55$ years). 
Backward Stepwise Logistic Regression followed by Normal Multiple Logistic Regression indicated that the outcome was influenced by several variables. Full and Reduced Models considered the masking effect of "Bleeding on Probing" and " $<25$ teeth present". Within the total population (35 - 87 years), increased age, a higher cigarette index, a greater plaque score and being single increased risk. When age stratified, younger adults (35 - 54 years) had an increased risk associated only with a higher cigarette index while older adults ( $\geq 55$ years) who were single or who had a family income $\geq \$ 25,000 /$ year were at increased risk.

\section{CONCLUSIONS:}

1. Univariate analysis indicated that being $\geq 55$ years or older was associated with $4 \mathrm{x}$ increased risk for a history of AP. Multivariate analysis showed a $1.06 \mathrm{x}$ increased risk with each year among the total study population.

2. A dose-response effect was observed with the cigarette-index. There was a $7.3 \mathrm{x}$ increased risk among the 35 to 54 year old heaviest smokers and $3.8 \mathrm{x}$ among the total study population. Those aged $\geq 55$ years had no apparent risk associated with smoking.

3. Being single increased risk in the total study population by $1.77 \mathrm{x}$ and $3.07 \mathrm{x}$ among those $\geq 55$ years. Single $35-54$ year olds were not at increased risk compared to those with partners.

4. Low income was not associated with increased risk.

5. There was a trend for $1.55 \mathrm{x}$ increased risk associated with $\geq 50 \%$ plaque. Multivariate analysis indicated no apparent increased risk for AP associated with gender, dental visiting, brushing / flossing, chronic conditions/ medications with potential drug action or life stress. 


\section{LIST OF TABLES}

4.1 Strength of the scientific evidence

$53-54$

6.1 Subject selection process

$71 \mathrm{a}$

6.2 Target population. Distribution of controls

73

6.3 Concordance of diagnostic criteria

84

6.4 Significant differences found between case and control according to explanatory variables

$85 a-b$ by diagnostic criteria

8.1 Distribution of cases and controls among target and study population by year of exam

8.2 Response rate to telephone interviews

8.3 Response rate to telephone interviews by interviewers

8.4 Reasons given for not participating in telephone interview

8.5 Radiograph types used to determine case-control status

122

8.5a Re-interview schedule

123

8.5b Test-retest reliability

123

8.5c Inter-examiner reliability 124

$\begin{array}{lll}8.6 & \text { Validity of interview } & 125\end{array}$

$\begin{array}{lll}8.7 & \text { "Quality" of interview } & 126\end{array}$

8.8 Univariate description of continuous variables 129a-b

8.9 Description of categorical variables

8.10 Collapsed categorical variables

$137-138$

8.11 Comparison of cases and controls: Crude disease- 140a-b exposure association for individual key explanatory variables 
8.12 Dose response to key explanatory variables $\quad$ 144a

8.13 Odds Ratios (unadjusted, age-gender adjusted) for key $\quad$ 145a explanatory variables

8.14 Dose response to key explanatory variables:

Age and gender adjusted

8.15a Full Model \#1 "Backward" Stepwise Multiple

152

Logistic Regression. Step One

8.15b Result of Backward Stepwise: Full Model \#1

153

8.15c Result of Normal Multiple Logistic Regression: 153

Full Model \#1

8.16a Reduced Model \#1 "Backward" Stepwise Multiple

Logistic Regression. Step One

154

8.16b Result of Backward Stepwise: Reduced Model \#1

154

8.16c Result of Normal Multiple Logistic Regression: 154 Reduced Model \#1

8.17 Explanatory variables: Comparison of cases and controls (Age 35-54 years)

$156 \mathrm{a}-\mathrm{b}$

8.18 Dose response to key explanatory variables (35-54 years). Reduced Model \#2a

$157 \mathrm{a}$

8.19a Full Model \#2a "Backward" Stepwise Multiple 158 Logistic Regression. Step One

8.19b Result of Backward Stepwise: Full Model \#2a 158

8.19c Result of Normal Multiple Logistic Regression: 158 Full Model \#2a

8.20a Reduced Model \#2a "Backward" Stepwise Multiple Logistic Regression. Step One

8.20b Result of Backward Multiple Logistic Regression: $\quad 159$

8.20c Result of Normal Multiple Logistic Regression: $\quad 159$ Reduced Model \#2a 
8.21 Explanatory variables: Comparison of cases and

$160 \mathrm{a}-\mathrm{b}$ controls (age $\geq 55$ years)

8.22 Dose response to key explanatory variables (age $\geq 55$ years): Reduced Model \#2b

8.23a Full Model \#2b "Backward" Stepwise Multiple

161

Logistic Regression. Step One

8.23b Result of Backward Stepwise: Full Model \#2b 162

8.23c Result of Normal Multiple Logistic Regression: 162 Full Model \#2b

8.24a Reduced Model \#2b "Backward" Stepwise Multiple 162 Logistic Regression. Step One

8.24b Result of Backward Stepwise Multiple Logistic 163 Regression: Reduced Model \#2b

8.24c Result of Normal Multiple Logistic Regression:

163 Reduced Model \#2b

9.1 Model parameters for final models 


\section{ACKNOWLEDGMENTS}

I would like to express my gratitude to the patients, faculty, staff and administration of the Faculty of Dentistry, The University of Manitoba for their cooperation with the study which forms the basis of this thesis.

To my thesis committee, Dr. T.K. Young, Dr. David Singer, Dr. M. Moffat, and Dr. Dexter Harvey, thank you for your guidance. Dr. Young, thank you for agreeing to supervise my program of studies and research. I appreciated your efficiency and admired your ability to focus on key issues. Dr. Singer, thanks for being an invaluable mentor. I found that your strong foundation in periodontology along with your research skills made tremendous contributions to the committee. Dr. Moffat, your attention to detail regarding study design was appreciated. Dr. Harvey, I found your suggestions on survey design and on presentation of conclusions very helpful.

Thank you to the members of the Faculty of Dentistry for your encouragement. In particular, thanks to Dean J.N. Wright and Associate Dean R.C. Baker for your early and continued support of my studies and research. Thank you to the members of the Department of Dental Diagnostic and Surgical Sciences for enduring the disruptions that my program undoubtedly caused.

Thank you to Mrs. Mary Cheang for your thoughtful statistical consultation and your approachable manner. I am also grateful for the cheerful, professional secretarial assistance provided by Ms. Mary Dudek who never complained about the numerous drafts or the more numerous tables. Computer data-base and programming assistance received from Mr. Darrell Backman was extremely helpful as was the chart and radiograph retrieval completed by Mrs. Helen Parker, Ms. Judy Bishop, Mrs. Pat Kolody and Mrs. Helen Janzen.

To my family, Dale, Tony and Timothy, thank you for encouraging me to pursue one of my dreams. I recognize the sacrifices that you have all made on my behalf over the past few years and I am truly grateful. (Yes, I still remember how to bake cookies!)

This research was supported in part by grants from the Manitoba Medical Services Foundation and the Faculty of Dentistry Dean's MRC fund. 


\section{CHAPTER ONE}

INTRODUCTION

\subsection{RESEARCH GOALS}

The research goals of this case-control study were:

a) to determine antecedent exposure of individuals with a history of periodontitis to specific health-compromising behaviors, medications, chronic diseases / conditions, socioeconomic and oral health variables.

b) to determine the presence and strength of association of antecedent risk indicators/markers with a history of periodontitis.

c) to test the unique effect of each variable on AP while controlling for the influence of the remaining variables.

\subsection{STATEMENT OF THE PROBLEM}

Periodontitis is a leading cause of tooth loss among adults (Weintraub and Burt 1985; Hunt 1988; Burt 1993; Oliver et al 1993). The influence of periodontitis on oral health and the quality of life is reflected not only in tooth loss; but also by inadequately functioning teeth. Discomfort, disability and disadvantage are frequent outcomes of periodontitis (Locker 1988, 1989, 1992). The cost of prevention and treatment of periodontitis by dental professionals is a public health issue of economic importance (Leake 1984; Pilot 1986; Sheiham 1991; Hollister and Weintraub 1993).

Recognizing the influence of oral health on the quality of life, the World Health Organization established a goal for the year 2000 "retention throughout life of a functional, esthetic, natural dentition of not less than 20 teeth and not requiring a prosthesis" (WHO, 1982). The prevalence of moderate adult periodontitis in North America is approximately $30 \%$ while 
advanced disease affects about $10 \%$ of the population (Griffiths et al 1988; Burt 1993; Brown et al 1993). Although periodontitis is not universal, retention of $\geq 20$ teeth appears to be jeopardized by this disease for a relatively large segment of the population.

Currently, there appears to be an inability to identify individuals or sub-populations most susceptible to periodontitis. Therefore, prevention and treatment of periodontitis are likely not being targeted effectively. Research into the etiology of periodontitis has been extensive. Adult periodontitis is believed to be caused by an interaction of environmental and host-defense factors (Wilton et al 1988; Griffiths et al 1988).

Attempts to identify variables which place individuals at increased risk for periodontitis suggest that bacterial dental plaque (Abdellatif and Burt 1987; Beck et al 1990; Brown et al 1990; Beck et al 1992) and smoking (Ismail et al 1983; Beck et al 1990, 1992; Horning et al 1992) are strongly associated with increased prevalence and severity of periodontitis, while the association of diabetes (Manouchehr-Pour and Bissada 1983; Hugson et al 1989; Emrich et al 1993; Locker and Leake 1993) and certain prescription medications (Knight and Wade 1973; Seymour and Heasman 1988; Thomason et al 1993) with periodontitis is ambiguous. The risk of periodontitis associated with hypertension and ischemic heart diseases (Quintarelli 1957; Grant and Bernick 1970; Lederman et al 1984; Patni et al 1985) has been inadequately studied.

There is increasing evidence that periodontitis, like other chronic diseases, is influenced by multiple risk factors (Bergstrom and FloderusMyrhed 1983; Bergstrom and Eliason 1987; Emrich et al 1993). However, relatively few of these studies have investigated pertinent behavioral and socioeconomic variables (Beck et al 1990; Hunt et al 1990; Levy et al 1987; Horning et al 1992; Locker and Leake 1993). 


\section{CHAPTER TWO}

\section{EPIDEMIOLOGY OF ADULT PERIODONTITIS}

\subsection{DEFINITION}

Adult periodontitis (AP) is a member of the family of periodontal diseases affecting those aged 35 years and older (Page et al 1983; Suzuki 1988). Periodontitis is inflammation of the gingiva, extending to the periodontal ligament, cementum and alveolar bone resulting in loss of clinical attachment (CAL) and bone (Suzuki 1988). Gingivitis, i.e., inflammation of the gingival soft tissue only, is another member of the family. The prevalence of gingivitis is $40-80 \%$ in North America and Europe (Kelly and Van Kirk 1965; Reddy et al 1986; Sooriyamoorthy and Gower 1989). This thesis focuses on AP. Rare gingival conditions and other forms of periodontitis such as refractory, rapidly progressive, prepubertal or juvenile periodontitis will not be addressed due to presumed differences in their etiology and natural history and their relatively low prevalence.

\subsection{PREVALENCE AND MEASUREMENT ISSUES}

Recent epidemiological reports suggest the prevalence of AP among industrialized nations ranges from $10-30 \%$ (Doll and Peto 1976; Hugson and Jordan 1982; Polson and Goodson 1985; Miller et al 1987; Pilot and Miyazaki 1991; Brown and Löe 1993). Cross-sectional studies have shown fairly consistent results: slight $\mathrm{AP}(1-3 \mathrm{~mm} \mathrm{CAL} \geq 1$ site $=99 \%)$; moderate $\mathrm{AP}(4-6 \mathrm{~mm} \mathrm{CAL} \geq 1$ site $=30 \%)$; advanced $\mathrm{AP}(\geq 7 \mathrm{~mm} \mathrm{CAL} \geq 1$ site $=10 \%)$ (Griffiths et al 1988; Burt 1993; Brown and Löe 1993). Variations in prevalence may be due to demographic/social differences among populations; as well as inconsistencies in indices, definitions, and measurement of disease. 
Prevalence is influenced by the cut-off points used to define periodontitis (Greene 1990; Brown and Löe 1993; Burt 1994). It has been suggested that $4-6 \mathrm{~mm}$ of CAL be the standard by which moderate periodontitis is defined and advanced disease involve $\geq 7 \mathrm{~mm}$ CAL (Burt 1991; 1994). Lower cut-off points overestimate prevalence (Burt 1994).

Studies prior to 1980 overestimated the prevalence of periodontitis in a number of additional ways. Early aggregated periodontal indices were influenced by misconceptions of disease progression and measurement errors (Griffiths et al 1988). Overestimation may also have resulted from sole reliance on probing depth (PD) (Griffiths et al 1988; Machtei et al 1992 Burt 1994) and measurement errors resulting from probing in the presence of inflammation (Listgarten 1980; van der Velden 1982; Badersten et al 1984; Griffiths et al 1988), use of excessive/uncontrolled probing pressure (van der Velden and DeVries 1978; Listgarten 1980; Polson and Goodson 1985; Griffiths et al 1988), inaccurate angulation/placement of probe (Listgarten 1980; Griffiths et al 1988).

Overestimation of prevalence may also have resulted from the predominant use of cross-sectional studies which could not distinguish a history of chronic AP from other forms of periodontitis such as localized/generalized juvenile periodontitis (LJP, GJP) or rapidly progressive periodontitis. However, a review of these conditions reports their prevalence to be relatively rare, e.g. LJP $=0.1-0.75 \%$ (Gelskey and Pruthi 1990).

Underestimation of the prevalence of AP may have occurred in studies employing partial-mouth examinations or where the incorrect unit of analysis was used (Hunt et al 1990), (e.g. mean PD/CAL score versus presence of attachment loss) or when PD was used exclusively to determine diagnosis (Griffiths et al 1988; Machtei et al 1992; Burt 1994), or if highly 
susceptible individuals were excluded from the study (Beck et al 1987; Miller et al 1987). Currently, CAL and PD are believed to be the best clinical estimates of prevalence/incidence of history of periodontitis (Griffiths et al 1988; Brown et al 1990; Burt 1991, 1994). Although the validity and reliability of the latter measurement is somewhat suspect (Badersten et al 1984; Jeffcoat and Reddy 1991; Caton 1993), both measurements are currently used in cross-sectional studies attempting to identify an individual's history of periodontitis (Listgarten 1980; Theil and Heany 1991; Caton 1993) and longitudinally, through repeated measures, to access disease progression (Bergstrom and Eliason 1989; Jeffcoat and Reddy 1991).

Radiographs have been used to increase the reliability and assess the validity of clinical measurements of periodontitis (Ryan 1985; Polson and Goodson 1985; Shrout et al 1990). While only repeated standardized radiographs are able to determine periodontal disease progression, conventional radiographs provide evidence of a history of periodontitis through evidence of alveolar bone destruction i.e., attachment loss (Hirschmann 1987). Radiographs have been used in clinical and epidemiological studies to estimate the prevalence of periodontitis (Bjorn and Holmberg 1966; Blankenstein et al 1978; Jenkins and Mason 1984; Kallestal and Matsson 1991). There is consensus that an alveolar bone crest located more than $3 \mathrm{~mm}$ apical to the cemento-enamel junction (CEJ) constitutes a history of periodontitis (Blankenstein et al 1978). However, radiographic diagnosis of incipient periodontitis is equivocal (Hausmann et al 1991; Caton 1993).

Problems associated with clinical and radiographic measurements of periodontitis have made epidemiological investigations challenging and their results often suspect (Burt 1994). Further discussion of the validity 
and reliability of clinical and radiographic measures employed in the current study are located in Chapter Seven, Eight, and Nine.

\subsection{SEVERITY, EXTENT, AND DISTRIBUTION}

Severe periodontitis ( $\geq 7 \mathrm{~mm} \mathrm{CAL/PD)} \mathrm{reflects} \mathrm{extensive} \mathrm{loss} \mathrm{of}$ periodontal support seriously jeopardizing tooth retention. While moderate periodontitis (CAL $=4-6 \mathrm{~mm}$ ) shows evidence of bone loss, the tooth's functional status is not immediately threatened (Burt 1994).

Severe AP is clustered among small subpopulations of adults (Löe et al 1978; Papapanou et al 1988; Okamato et al 1988; Burt 1991, 1994). Among those with the highest prevalence/severity of periodontitis are the elderly ( $\geq$ 60 years) (Hugson and Jordan 1982; Miller et al 1987; Beck et al 1990; Hunt et al 1990; Pilot and Miyazaki 1991). It may be appropriate to consider "severity for age" for different age groups to reflect the dentate elderly's resistance to disease and longevity of functional demand required for younger populations (Beck et al 1990). Prevalence of AP is higher among males (Beck et al 1987; Miller et al 1987) and non Whites (Kelly and Van Kirk 1965; Doll and Peto 1976; Horning et al 1992; Machtei et al 1992; Aday and Forthofer 1992) those with high levels of plaque (Kelly and Van Kirk 1965; Kelly and Harvey 1979; Miller et al 1987; Machtei et al 1992) and low income/education (Kelly and Van Kirk 1965; Nikias et al 1975; Aday and Forthofer 1992); as well as those with limited access to dental care (Kelly and Van Kirk 1965; Nikias et al 1975) and those who smoke (Bergstrom 1989; Horning et al 1992; Locker and Leake 1993) or have compromised health, e.g. diabetes (Wilton et al 1988; Hugson et al 1989;Emrich et al 1993). Many of the reviewed reports were from cross-sectional studies which failed to consider the potential influences of multiple confounding 
variables. As a result, the effect of these factors may have been over or underestimated.

Among employed adults in the United States in 1985, an average of 3.4 sites per person were affected by moderate periodontitis and less than one site with advanced periodontitis (Brown et al 1990). There may have been an underestimation of extent however due to use of half-mouth examination.

International longitudinal and cross-sectional studies have shown that some populations (Cutress et al 1982; Africa et al 1985) / individuals (Löe et al 1986) are apparently resistant to periodontitis despite exposure to abundant plaque and experiencing a high prevalence of gingivitis (Africa et al 1985). A 15-year longitudinal study of the natural history of periodontitis was conducted among a homogeneous, caries-free Sri Lankan population which had never experienced dentistry. Abundant plaque and a high prevalence of gingivitis existed; however, three distinct groups of individuals were identified including one which had no progression to periodontitis (Löe et al 1986). Such variations in periodontal disease progression in an otherwise homogenous population have stimulated investigations of host susceptibility and the influence on AP of a variety of potential risk factors.

\subsection{NATURAL HISTORY}

\subsubsection{Disease Initiation}

Several periodontal indices, developed approximately three decades ago, have been used to study gingivitis and periodontitis in numerous crosssectional studies (Kelly and Van Kirk 1965; Kelly and Harvey 1979; Miller et al 1987; Beck et al 1987; Brown et al 1990). Through these studies and more rigorous experimental designs, it has been demonstrated that bacterial 
dental plaque causes gingivitis and its removal results in the resolution of gingivitis (Löe et al 1965; Lindhe et al 1973; Axelsson and Lindhe 1978; Newman 1990).

A comprehensive review article describes the initiation and progression of gingivitis and periodontitis (Page 1986). Within about four days of undisturbed plaque accumulation, the "initial lesion" develops in a previously healthy site. Approximately three days later, the "early lesion" is observed wherein ulceration of the sulcular epithelium occurs, allowing introduction of microbial substances into underlying connective tissues. The "established lesion" follows. Periodontitis has been designated the subsequent "advanced lesion".

The precise transition from healthy gingiva to gingivitis is not fully understood; but, even less clear is the transition from gingivitis to periodontitis. Established lesions of two types have been described: those that remain stable i.e., not progressing for months/years and those which convert to progressive destructive periodontitis. Currently, it is impossible to distinguish gingivitis lesions with progressive potential from those which are more innocuous (Page 1986; Suzuki 1988).

\subsubsection{Disease Progression}

Progression in some sites/individuals may be due to: 1) aberrations in the host cell-responsiveness to plaque and be related to immune hyperresponsiveness or deficiencies or 2) colonization infection by highly pathogenic bacteria causing direct tissue destruction (Suzuki 1988).

Progression of periodontitis has been described as "continuous". However, this description is based on cross-sectional epidemiological studies which have observed increased attachment loss associated with age, suggesting that individuals lost from 0.03 to $3.11 \mathrm{~mm}$ attachment per 
year (Goodson 1986). The "continuous" theory has been challenged recently by results from longitudinal studies. Prospective repeated clinical measurements have shown "episodic bursts" of periodontal destruction and remission (Page 1986; Goodson et al 1982; Haffajee et al 1982).

Prospective studies are best able to assess disease progression (Clerehugh et al 1988; Burt 1994). Progression or 'active' disease has been defined as loss of 0.4 to $3 \mathrm{~mm}$ CAL over a one year period (Goodson 1986; Jeffcoat and Reddy 1991; Burt 1994). Some sites in an individual's mouth may follow a continuous pattern of destruction while others may undergo episodic bursts. Studies employing repeated clinical measurements could, due to inappropriate time intervals between measurements, mask evidence of "episodic" activity (Jeffcoat and Reddy 1991).

A 15 year longitudinal study of the progression of periodontitis among a homogeneous population of caries-free Sri Lankans showed that although plaque and gingivitis were abundant among all individuals in the population, there were three distinct rates of disease progression: rapid, affecting $8 \%$; moderate, affecting $81 \%$; while no destruction was observed in $11 \%$ of the population (Löe et al 1986).

Future longitudinal studies should attempt to clarify the natural history and progression of periodontitis and concurrently investigate the influence of suspected risk factors on disease initiation/progression.

\subsection{PUBLIC HEALTH IMPACT}

Whether periodontitis is considered a public health problem depends on the oral health goals of the population. The World Health Organization's (WHO) oral health goal for the year 2000 is, "the retention throughout life of a functional esthetic, natural dentition of not less than 20 teeth and not requiring a prosthesis" (WHO 1982). This goal seems to be in jeopardy for a 
substantial proportion (10-30\%) of adults who experience periodontitis. Only recently has the possibility of AP predisposing individuals to other diseases such as coronary heart disease been considered (Destefano et al 1993; Page 1995).

\subsubsection{Economic Costs and Lost Productivity}

The impact of periodontitis can be estimated in terms of social economics and productivity. In $1985,6.4 \%$ ( $\$ 27.5$ billion) of the total health bill in the United States was spent on dental care. Of that, $1 \%$ ( $\$ 275$ million) was spent on treating periodontal disease, with substantially more spent on clinical prevention and prosthetic replacement of the teeth lost to periodontitis (ADA 1987). If the estimated 25 million diseased but untreated Americans would have received periodontal care in 1985, the cost would have been $\$ 7$ billion (Polson and Goodson 1985). The total expenditures for dental services in the United States in 1991 was $\$ 37.1$ billion. Expenditures are predicted to increase to $\$ 62.3$ billion by the year 2000 (Bolden et al 1993) due to an aging population and increased tooth retention.

The economic consequences of dental disease to the individual are significant since over half of North Americans are not covered by dental insurance or third party pre-payment plans (Miller et al 1987). Those not covered typically have the greatest dental/periodontal needs (Miller et al 1987). Canadians spent $\$ 53.51$ per capita for dental care in 1980 which constituted $47 \%$ of their out-of-pocket health care expenditures (Leake 1984). On average, employed adults in the United States lost 1.48 work-hours/year in 1989 due to dental visits/problems, resulting in reduced income for the individual and productivity for society-at-large (Gift et al 1992). In addition 
to economic losses, approximately 117,000 school hours/100,000 students were lost in 1989 due to dental visit/disease (Gift et al 1992).

\subsubsection{Influences on Quality of Life}

The impact of periodontitis extends beyond economic issues. Oral health is an integral component of general health and influences quality of life (Dolan and Atchison 1993). Periodontitis and its consequences diminish the quality of life by causing tooth loss (impairment) which results in functional limitation and discomfort. Individuals may consequently experience restrictions in their eating and limitations in their social roles (Locker 1992). Although less is known about the extent to which those affected by $\mathrm{AP}$ are handicapped, it is plausible that loss of function and esthetics impact on social acceptance. Disadvantage may subsequently occur if life chances are affected, such as loss of opportunity occupationally or socially (Locker and Grushka 1987; Locker 1989, 1992; Hollister and Weintraub 1993). The impact of periodontal disease and related tooth loss on systemic health has not been adequately studied.

\subsection{CONCLUSIONS}

Although moderate-advanced periodontitis is not life-threatening, its prevalence in North America (10-30\%) is as high as or higher than a number of other chronic diseases and conditions such as diabetes, 5\% (Reeder et al 1982) and hypertension, 14\% (Young et al 1991; Joffres et al 1992). Page (1995) suggests that further investigation of general health implications of periodontitis should be conducted. The economic impact of periodontitis on the individual and society is substantial as is its influence on the quality of life. Costs for preventing and treating periodontitis are high due to the predominant use of the dental (medical) model. Currently it 
is not possible to identify those most susceptible to AP which may result in overtreatment of many individuals and undertreatment of those in greatest need.

With these public health concerns in mind, and armed with an improved understanding of the natural history of AP, and powerful multivariate statistical methods, epidemiologic attention should attempt to identify factors, indicators, markers and determinants of AP. Once identified, future prospective studies could investigate how these variables influence the risk and progression of AP. The ultimate goal is to better target programs of prevention and treatment at those with greatest need/risk. 


\section{CHAPTER THREE}

\section{RISK ASSESSMENT}

\subsection{CONCEPTUAL ISSUES}

"Risk" refers to the probability of an event occurring (MacMahon and Pugh 1970; Schlesselman 1982; Forbes and Thompson 1989; Bader 1990). Epidemiological studies are conducted to quantify risk and to identify variables that are associated with, or cause, disease.

\subsubsection{Relative Measures of Disease Occurrence}

Two methods of quantifying risk are: 1) Relative Risk (RR) which estimates magnitude, i.e., how much more likely disease occurs in the exposed compared to the unexposed in a prospective cohort study. If the RR differs from unity, then the variable of interest is associated with an increased risk of disease (Schlesselman 1982; Kelsey et al 1986; Hennekens

and Buring 1987; Kahn and Sempos 1989); 2) The Odds Ratio (OR) is a measure of association which, for rare diseases approximates the RR. The $\mathrm{OR}$ is the ratio of the odds of disease in exposed individuals relative to those unexposed (Schlesselman 1982; Kelsey et al 1986; Hennekens and Buring 1987; Kahn and Sempos 1989). The current study assessed the odds ratio of potential explanatory variables.

\subsubsection{Risk Factors, Markers, Indictors and Determinants}

A variety of terms related to risk are used in the literature.

1) A "risk factor" has been defined as "an aspect of personal behavior or lifestyle, an environmental exposure, or an inborn or inherited characteristic, which, on the basis of epidemiological evidence is known to be associated with health-related conditions" (Last 1988). A more stringent definition requires that the variable has been shown 
to be causal through human random controlled trials (RCT) or community trials (Bader 1990). Burt (1994) suggests that to qualify as a risk factor, the variable must also be modifiable, e.g. plaque control.

2) "Risk Indicators" are variables associated with disease experience which are assumed, on theoretical grounds, to play a causal role in the disease process (Locker and Leake 1993). Risk indicators are variables that have been associated with periodontitis through crosssectional/case control studies but have yet to demonstrate causality through prospective studies, e.g. cigarette smoking (Bader 1990).

3) “Risk Markers" are attributes/exposures associated with an increased probability of a condition/disease occurring but which are not assumed to be causal (Last 1988). They are characteristics which identify groups most likely to have or have had disease e.g. bleeding on probing (Abramson 1990; Locker and Leake 1993).

4) "Determinants" are attributes or exposures that increase the probability of disease but which can not be modified, e.g. age, race (Burt 1994). Determinants are difficult to classify because attributes such as education, culture, and poverty are theoretically but unrealistically modifiable (Burt 1994).

Risk assessment has been hampered by conflicting definitions and inaccurate use of risk-related terminology and scientific methodology in the literature. The result has been inconsistent and misleading reports of the risk factors for periodontitis.

\subsubsection{Cause versus Association}

There is a distinct difference between cause and association. "Cause" is defined as an event, condition or characteristic that has an essential influence on producing a disease (Rothman 1986). The term "risk factor" 
rather than "causative agent" is generally used to study chronic disease since "risk factor" emphasizes the complexity of interactions among multiple exposures i.e., the "web of causation".

"Association" refers to dependence between two or more variables. Association may be tested statistically; however, a significant association does not imply causation but only eliminates variability due to chance, as an explanation for the association (Schlesselman 1982; Kelsey et al 1986).

Several categories of association exist: 1) independence i.e., not statistically associated; 2) secondarily associated, i.e., statistically associated but not causal; 3) statistically associated and indirectly causally associated; and 4) statistically associated and directly causal (MacMahon and Pugh 1970). The periodontal literature contains many reports of the first three types of association between disease and a variety of individual risk variables. Few however claim a causal association. An assessment of the strengths and weaknesses of studies of causality and association is made in Chapter Four.

\subsubsection{Risk Assessment}

The previously described concepts and principles of risk are applied directly in the process of risk assessment. Risk assessment is used in making health-policy decisions and generally considers several issues: 1) The magnitude of the risk as indicated by the previously described RR or OR. The larger the magnitude, the less likely the association could be explained by another uncontrolled variable; 2) The strength of the scientific evidence supporting the association between risk and disease. The more consistent the cross-sectional, prospective and experimental results, the stronger is the support for the association; 3) The size of the population at risk, as obtained from national epidemiological studies. The more prevalent 
the disease, particularly if it is serious, the more likely interventions should be made; 4) The ease of modification of the risk-related exposure or behavior (Schlesselman 1982; Rothman 1986; Forbes and Thompson 1989). Certain variables (gender) are not modifiable or difficult (poverty) to modify while others such as smoking may be modified. Modification of risk is addressed more fully in Chapter Nine.

\subsubsection{Models and Criteria for Causation}

In the absence of plentiful experimental data, observational, quasiexperimental and cross-sectional data are useful in investigating causality (MacMahon and Pugh 1970). Since the 19th century when the Henle-Koch postulates were introduced, there has been an historical evolution of the concept, "causality". The Henle-Koch postulates have limited validity and reliability due to their failure to consider multiple causation, the asymptomatic/carrier or the biologic spectrum of disease among other shortcomings (Evans 1978; Schlesselman 1982; Kelsey et al 1986). Sir Bradford Hill (Hill 1965; Schlesselman 1982) outlined the following nine criteria which have been used extensively to investigate the strength of the scientific evidence to support an inference of a causal relationship:

1. Strength of Association: The larger the RR or OR the less likely the association is spurious. It has been noted however that just because an association appears weak, causality should not be ruled out because strength may be dependent on the relative prevalence of other variables (Rothman 1986).

2. Consistency: Repeated observation of association observed under varied conditions. Lack of consistency however does not rule out 
causality because some effects are produced by their causes only under specific circumstances (Rothman 1986).

3. Specificity: A cause is specific to an effect if the introduction of the causal factor is followed by the effect and if removal of the factor results in resolution of the effect (Hill 1965). Some believe that this criterion is weak and meaningless due to its simplistic proposition of single-factor causation (Schlesselman 1982; Rothman 1986).

4. Temporality: A causal relationship requires that the factors believed to cause a disease must precede the disease. This temporal sequence is often difficult to ensure with chronic diseases in which progress is continuous, often of long duration and modulated by the host. This criterion is considered by many to be a sine qua non for causation (Hill 1965; Rothman 1986).

5. Biologic Gradient: A dose-response curve supports causality and is seen when an increase or decrease in exposure to a risk factor corresponds to an increase or decrease in the frequency and/or severity of disease. A dose-response trend does not however necessarily indicate causality. Confounders may produce the apparent trend (Rothman 1986).

6. Biologic Plausibility: The suspected cause should be biologically credible. However, it is important to note that plausibility is based on current biological knowledge (Schlesselman 1982).

7. Coherence: Causality is supported if the causal relationship between exposure and outcome does not conflict with known facts regarding 
the natural history of the disease (Schlesselman 1982). It has been suggested however that lack of coherence should not singularly nullify a casual hypothesis (Hill 1965).

8. Analogy: A causal relationship becomes more believable if the exposure has been shown to have a similar effect on a related condition (Schlesselman 1982).

9. Experiment: i.e., the strongest support for causation is empirical evidence whereby the mechanism which produces the outcome may be stated (Hill 1965; MacMahon and Pugh 1970; Schlesselman 1982).

\subsubsection{Conclusions}

Once established that the prevalence of a disease is a public health concern, the next step in risk assessment is to determine the strength of the scientific evidence supporting the association between a variable and disease. In the current study, this was accomplished through a review of the literature. Hill's criteria of causation formed the framework for the analysis (Chapter Four). The reported magnitude of risk for these key explanatory variables was determined and ultimately compared to the odds ratios calculated from the current case control study (Chapters Eight and Nine). Modification of risk-related behavior/exposure is discussed in Chapter Nine. 


\section{CHAPTER FOUR}

\section{STRENGTHS OF THE SCIENTIFIC EVIDENCE}

\subsection{THEORETICAL FRAMEWORK}

Evidence regarding potential risk factors, indicators, markers and determinants for adult periodontitis (AP) has been derived predominantly from cross-sectional or case-control studies. This is particularly true for most social and behavioral factors (Bader 1990). As is discussed in Chapter Five, "Design Options For Risk Assessment", controlled prospective clinical and community trials are ideal designs for identification of risk factors because they address temporality and control extraneous and confounding variables (Bader 1990). Where available, these studies were evaluated. However, their sparsity in the literature necessitated the application of an alternate method of assessing the strengths and weaknesses of the literature in support of an association between AP and the following variables: age, gender, education, income, race, cigarette smoking, diabetes, hypertension, ischemic heart disease, use of specific medications, and plaque.

The theoretical framework of analysis in this study was adapted from Bradford Hill's Criteria for Causation (Hill 1965). The criteria are described in Chapter Three, Section 3.1.5, "Models and Criteria for Causation". Although not designed by Hill to be used as criteria for causation, when met, the criteria support an inference of a causal relationship (Hill 1965; Schlesselman 1982; Rothman 1986). Results of the analysis of the supporting evidence for each variable are provided in Table 4.1. The results are based on the following critical review of the literature. 


\subsection{SOCIODEMOGRAPHIC VARIABLES}

\subsubsection{Age}

Biologic Plausibility - A recent review of the literature concluded that although the prevalence and severity of periodontitis increases with age and despite the fact that the aging process itself may account for some physiological changes, the aging process itself does not cause periodontal destruction (van der Velden 1994). Changes in plaque composition and altered tissue response to plaque may occur with age. Although neither theory has been demonstrated empirically, it may be that the local environment is altered through recession, prompting ecological alterations in the flora or that periodontal inflammation develops more rapidly among the elderly or that they respond more slowly to wound healing.

Generally, individual susceptibility has not been controlled in most studies and the influence of reduced salivary function or increased use of medications in the elderly is rarely considered. Local factors may confound the issue when investigating diminished immune response with age. The cohort-effect of different age groups is also often overlooked.

The apparently greater prevalence/severity among the aged has been attributed to the accumulative process of AP over an individual's lifetime (van der Velden 1994). It has been suggested that a thinning of the epithelium and diminishing of keratinization and other morphologic cell changes occur with age but these changes have not been demonstrated consistently. Animal studies have shown a decreased rate of collagen synthesis with age. A greater apposition of cementum with age may cause passive eruption and influence the periodontium through physiological apical migration. The width of the cribiform plate of alveolar bone 
decreases with age in monkeys although this has not been shown in man (van der Velden 1994).

Although the suggested effects of age on AP are biologically plausible, it is believed that higher levels of plaque and the cumulative effect of the disease account for the high prevalence/severity observed in the elderly rather than the aging process itself (Abdellatif and Burt 1987).

Consistency - Many cross-sectional studies have reported an association between age and periodontitis. This association was observed in a periodontitis-resistant population (Baelum et al 1986) as well as in crosssectional clinical studies (Papapanou et al 1988; Machtei et al 1992) and among elderly residents of Ontario (Locker and Leake 1993) and Iowa (Hunt et al 1990) and in representative national United States surveys (Kelly and van Kirk 1965; Miller et al 1987; Brown et al 1990). Longitudinal studies have also found an association with age and the development of periodontitis (Grbic et al 1991; Haffajee et al 1993). However, plaque was likely confounded with CAL at baseline. Most studies fail to consider temporality, the complex multifactorial nature of periodontitis and other confounding/ extraneous variables. Representativeness of study populations was usually suspect and the use of partial mouth recording could have resulted in under/overestimation of prevalence/severity. The latter however is unlikely to have influenced differences observed among age groups.

Review articles have concluded that age is a correlate rather than a risk factor for periodontitis (Burt 1994; van der Velden 1994). Re-analysis of cross-sectional data from the United States demonstrates that regardless of age, $\geq 95 \%$ of those with good oral hygiene did not have periodontitis, also supporting age as correlate rather than a cause of periodontitis (Abdellatif 
and Burt 1987). Some studies have shown that CAL is not inevitable with age when plaque is adequately controlled (Ismail and Lewis 1993).

Biologic Gradient - A biologic gradient between age and periodontitis has been suggested from results of cross-sectional studies. These studies show that as age increases so does the prevalence/severity of periodontitis. Among Tanzanians, the prevalence of AP among those aged $30-34$ years was $10 \%$, while among 35 - 39 years $14 \%, 40-49$ years $19 \%$ and $\geq 50$ years $31 \%$ (Baelum et al 1986). Other studies have shown that slight AP affects only $5 \%$ of 15 year olds but $80 \%$ of 60 year olds and advanced AP is seen in $0.1 \%$ and $15 \%$ respectively (van der Velden 1991). These findings are supported by other cross-sectional studies (Kelly and van Kirk 1965; Miller et al 1987; Brown et al 1990) as well as by longitudinal data (Löe et al 1986). Most studies however fail to consider confounding variables, such as plaque or the multifactorial nature of $\mathrm{AP}$ or its cumulative affect with aging.

Strength - The observed OR $=1.24$ (95\% confidence interval, $1.24-1.28)$ for the association of categorically-grouped age with periodontitis is relatively weak considering that in the same analysis of cross-sectional data, there was an $\mathrm{OR}=20.52(95 \%$ confidence interval $17.75-23.72)$ for categoricallygrouped plaque, suggesting that age is more likely a correlate than a risk factor for AP (Abdellatif and Burt 1987).

Specificity - Various types of periodontitis are found in children (prepubertal) and youth (localized/generalized juvenile periodontitis) and there is some evidence that chronic periodontitis may affect individuals as young as eight years of age (van der Velden 1991). At the opposite end of the age spectrum, when plaque is controlled, periodontitis does not occur in 
most elderly individuals (Ismail and Lewis 1993). Therefore, the specificity of periodontitis with age is not supported.

It is suggested that rather than a specific "age-effect", the association between age and AP may be due to a "cohort-effect" affecting their attitudes, beliefs and behaviors toward dentistry (Ettinger 1992). The "old-elderly" cohort grew up when dentistry was a luxury and when the treatment for most dental disease was extraction. Prevention was not stressed. Today's "old-elderly" tend to have lower levels of education and income than the general population. Both factors have been associated with decreased utilization of dentistry and increased prevalence of periodontitis (Walden 1988; Ettinger 1992; Haffajee et al 1993). Today's "new-elderly" cohort, on the other hand, are more dentally-aware, have a higher income/education, visit the dentist more regularly, are more likely to retain their teeth and take preventive actions more consistently (Ettinger 1992; Locker and Leake 1993).

Coherence - Although an increase in prevalence/severity of AP with age appears to concur with our current understanding of host susceptibility as well as with the natural history of disease, related crosssectional/longitudinal studies have generally failed to consider confounding variables, or the multifactorial complexities of AP (Löe et al 1986; Baelum et al 1986; Haffajee et al 1993). The lack of sufficient prospective empirical evidence in man limits the understanding of any influence of age on the natural history of AP (van der Velden 1994).

Temporality/Analogy/Experiment - There was no evidence to support these criteria. 


\subsubsection{Gender}

Consistency - Most longitudinal studies show an association between gender and the development of AP (Machtei et al 1992). However it has been shown that males also had higher levels of plaque, which confounds the gender issue. Two longitudinal studies failed to show a difference in AP between genders (Grbic et al 1991; Haffajee et al 1993). However, these two studies had small sample sizes or were of short duration which may not have permitted gender differences to be found.

Cross-sectional studies consistently have shown a higher prevalence/severity of periodontitis among males. Gender difference was evident among Tanzanian adults (Baelum et al 1986) and elderly Iowa residents (Hunt et al 1990) as well as in a military population (Horning et al 1992) and representative national United States surveys (Kelly and Van Kirk 1965; Miller et al 1987; Brown et al 1990). However, males also had more plaque in these studies, confounding the apparent relationship. In addition, males also had more teeth present than females which, along with excluding the edentulous (more likely to be females) would bias the magnitude of the difference in prevalence between genders. The military population was predominantly male (85\%) i.e., unbalanced which possibly resulted in an underestimation of AP among women, particularly if the participating women in the study were younger than the males.

Temporality - Males have been shown to have a higher prevalence of periodontitis. However gender is not a direct cause of AP (Beck 1984).

Specificity - Psychosocial factors related to gender may explain differences in AP between genders. Social norms are believed to influence the male tendency for taking more risks such as smoking, having less health-related knowledge and taking fewer preventive actions (Coburn and Pope 1974; 
Blaikie 1979; Locker 1989; ). It is these factors which likely influence gender differences in AP rather than sex specifically.

Biologic Plausibility / Gradient / Strength / Coherence / Analogy /Experiment - There was no evidence to support these criteria.

\subsubsection{Education, Income, and Race}

Consistency - Longitudinal and cross-sectional studies have shown inconsistent results regarding the association between race or lower levels of education/income and increased prevalence/severity of AP. A six month longitudinal study of risk indicators for progressive AP failed to find an association with income/education or race (Grbic et al 1991). However, sample size $(n=75)$ was relatively small and the duration rather short (six months) to expect to find significant differences. Failure to find association could result if account was not made for differences in the number of missing teeth at baseline since partial and complete edentulousness is more common among lower SES and minority groups.

Cross-sectional studies of representative United States national samples have consistently shown increased AP among those of lower education/income (Kelly and Van Kirk 1965; Bailit and Manning 1985; Miller et al 1987; Brown et al 1990). Similar results have been observed among elderly Ontario residents (Locker and Leake 1993), and among New York residents (Nikias et al 1975).

Increased prevalence/severity of AP are similar when racial minorities such as elderly North Carolina Blacks (Blaikie 1979; Beck et al 1990; Horning et al 1992; Machtei et al 1992) and United States racial groups (Miller et al 1987; Brown et al 1990) have been studied. Cross-sectional studies also indicate however that there was poorer oral hygiene and less 
preventive behavior among these low SES groups (Nikias et al 1975; Chen and Stone 1983; Brown et al 1990; Syrajala et al 1992) and among non-Whites (Machtei et al 1992). When race was controlled, the level of education was found to explain differences in preventive dental knowledge and behavior observed among certain racial groups (Soh 1993).

Lower income groups have been found to practice less regular oral hygiene (Chen and Stone 1983), although reported toothbrushing frequency has not consistently been shown to vary among various SES groups (Nikias et al 1975). This inconsistency may be due to social desirability bias and over-reporting of brushing or to inadequate brushing performance.

There is uncertainty as to whether many of the cross-sectional studies were actually representative of the target populations since some of the most susceptible individuals were excluded. The most recent United States national probability survey of the employed and seniors excluded the unemployed and self-employed. This flaw could have resulted in an underestimation of the association between lower SES groups and AP.

Most studies of race and SES failed to control for confounding variables or consider the complex multifactorial nature of AP (Blaikie 1979). Confounding is evident among those studies in which racial differences disappeared when education and plaque were controlled (Kelly and Van Kirk 1965). When analyses accounted for education, the trend of poorer periodontal health remained significant among low income groups (Kelly and Van Kirk 1965; Miller et al 1987; Brown et al 1990). While education and income have been shown to be closely related, they do not always have the same health implications. Income implies the ability to purchase dental care while education implies a greater awareness/knowledge of what appropriate care is or should be. 
Specificity - Income, education, and race do not influence the prevalence/severity of periodontitis directly. Instead, the association of these variables with factors such as oral hygiene practices, dental visiting and health-compromising behaviors have a more direct impact on AP (Nikias et al 1975). This theory is supported by the social science literature wherein the influence of social class and psychosocial variables on health attitudes and behavior is fairly well established (Bullough 1972; Coburn and Pope 1974; Blaikie 1979; Wilson and Eliason 1981; Locker 1989).

Analogy - There is a higher prevalence/severity among lower SES and minority groups of many dental conditions such as edentulism, caries and oral cancer (Beal 1989). However the influence of SES and race on these outcomes is once again, indirect.

\section{Biologic Plausibility / Gradient / Temporality / Strength / Coherence /}

Experiment - There is no evidence to support these criteria.

\subsection{HEALTH-COMPROMISING BEHAVIOR}

\subsubsection{Cigarette Smoking}

Biologic Plausibility - The biologic plausibility of the relationship between cigarette smoking and AP is strong and supported by a number of studies.

Cross-sectional studies among 145 patients with severe periodontitis showed no difference between smokers and non-smokers regarding presence of certain periodontopathogens namely, Actinobacillus actinomycetemcomitans, Bacteroides gingivalis and Bacteroides intermedius (Preber et al 1992). The laboratory tests used had only poor to moderate $(0.2-0.6)$ reliability due to sampling/culturing difficulties. In 
addition, unknown periopathogens may play a key role in periodontitis among smokers.

Longitudinal laboratory studies have demonstrated that nicotine disturbs the alignment of gingival fibroblasts to glass and to human root surfaces in vitro (Raulin et al 1989). The generalizability of these findings is unknown. Nicotine has been shown to produce a severe reduction in intraarterial flow rates in a rabbit model (Clarke et al 1981). It has been suggested that the nicotine may cause ischemia of the periodontal tissues, particularly since the vessels which deliver blood to the gingiva are endarterioles and there is no collateral circulation to the papillae (Clarke et al 1981). Prospective human studies have shown that the vascular response among smokers to plaque-induced gingivitis is suppressed when compared to nonsmokers (Bergstrom et al 1988). These results could be due to the pharmacological effect of tobacco smoke on circulation resulting in vasoconstriction and decreased circulation (Clarke et al 1981). These vascular changes could explain why smokers often exhibit less gingivitis than nonsmokers (Feldman et al 1983; Preber and Bergstrom 1985, 1986; Bergstrom et al 1988; Danielson et al 1990).

A three-day quasi-experimental prospective study demonstrated that smoking one cigarette immediately before cell collection resulted in a significant decrease of oral polymorphonuclear leukocyte vitality and ability to phagocytize (Kenny et al 1977). The study failed however to control for potential differences in plaque among smokers/nonsmokers. Another quasi-experiment demonstrated that one cigarette produces a sufficient amount of toxic material in the oral cavity to inhibit completely the function of exposed oral leukocytes (Eichel and Shahrik 1969). The latter was a shortterm, unreplicated study. Another quasi-experiment of young, periodontally healthy individuals failed to show an impaired response of 
PMNs (Kraal and Kenny 1979). This inconsistency with other studies could be due to subjects' young age and minimal plaque as well as difference in study methodology. The results of another quasi-experiment suggest an immunosuppressant effect by the combustion products of tobacco as measured by salivary immunoglobulin A levels (Bennet and Read 1982). These results support other similar studies (Costabel et al 1986). A longitudinal cohort study found the bone mineral content among smokers to be $10-30 \%$ lower among smokers compared to nonsmokers (Rundgren and Mellstrom 1984). The authors speculated that tobacco may disturb the metabolism of vitamin D or influence hormonal states. Studies have strongly supported the biologic plausibility of a local and/or systemic effect of cigarette smoking on AP.

Consistency - The literature is consistent in reporting association between cigarette smoking and AP, regardless of study design or population. Crosssectional studies which measured smoking and AP concurrently, show an increased prevalence/severity of AP among smokers within elderly North Carolina residents (Beck et al 1990, 1992), non-institutionalized elderly Iowa residents (Levy et al 1987), elderly Ontario residents (Locker and Leake 1993) among a military clinic population (Horning et al 1992), between co-twins, one of whom smoked (Bergstrom and Floderus-Myrhed 1983), among Swedish dental hygienists (Bergstrom et al 1991), among individuals with diabetes (Haber et al 1993), in a representative United States survey (Ismail et al 1983), among a population which practiced good oral hygiene (Bergstrom and Eliason 1987), among a Swedish population (Preber and Bergstrom 1986), among patients attending a dental-teaching clinic (Bergstrom 1989), and veterans' administration hospital patients (Feldman et al 1983). 
On the other hand, studies tend to show a lower prevalence of gingivitis among smokers even though smokers often tended to have greater levels of plaque Sheihiam 1971; Bergstrom and Floderus-Myrhed 1983; Preber and Bergstrom 1985, 1986). Healing following periodontal surgery is delayed among smokers (Preber and Bergstrom 1990). The latter study however consisted of a small sample and the differences found could have been explained by clinical measurement error.

Treatment needs of smokers have consistently been shown to be greater than non-smokers although treatment-need included stain/calculus removal (Goultschiin et al 1990). The former requiring more an esthetic than therapeutic procedure. One large cross-sectional British study failed to find a difference in prevalence of AP between smokers/nonsmokers when plaque level was controlled (Sheihiam 1971), although plaque level was significantly higher among smokers as had been observed previously (MacGregor 1984; Preber and Bergstrom 1985). Others have found plaque scores to be no different between smokers and non smokers (Feldman et al 1983; Preber and Bergstrom 1986; Bergstrom and Eliasson 1987). One study showed less plaque among smokers compared to nonsmokers (Feldman et al 1983). Some cross-sectional smoking studies excluded edentulous and other highly susceptible individuals which could have biased the results, potentially underestimating the association between AP and smoking. Some studies failed to account for confounders such as plaque, age, race, and socioeconomic status (SES) which could also have resulted in study bias and under/over estimation of association.

A co-twin study on smoking and periodontitis was able to effectively control for many of the key variables and potential confounders such as genetics, gender, age. As a result, the study has strong validity (Bergstrom and Floderus-Myrhed 1983). However, results could have been affected by 
recall and social desirability bias, clinical measurement errors and lack of blinding. Certain highly motivated homogenous populations were not representative of the general population (Bergstrom and Eliasson 1987; Bergstrom et al 1991) although some confounding variables had been controlled through regression analysis (Bergstrom and Floderus-Myrhed 1983; Bergstrom and Eliasson 1987).

Two case-control studies showed that smoking was significantly more prevalent among those with periodontitis than those with a healthy periodontium even after controlling for plaque (Bergstrom 1989; Haber and Kent 1992). However these studies did not evaluate other potential confounders in the populations' demographics/ SES characteristics nor could they establish temporality. Cases and controls were not selected from the same population in either study which could have introduced bias.

Biologic Gradient - There is indirect evidence of a gradient in exposure to smoking when current smokers are compared to previous smokers and never smokers. The first have the highest prevalence of AP (Bergstrom et al 1991). Those who smoke most heavily have a higher prevalence/severity of disease (Preber and Bergstrom 1986; Feldman et al 1983) than those who smoke less. Less consistent with the literature are the results of a national United States survey which showed no gradient effect (Ismail et al 1983). This inconsistency could be due to inaccuracies and biases in self-reporting and to differences in study design.

Temporality - There is no evidence to support this criteria as no prospective cohort study has been conducted. Experimental imposition of smoking on the population would be unethical. 
Strength - The magnitude of the association between AP and smoking has been shown in several studies. A cross-sectional study has demonstrated an increased $\mathrm{OR}=2.9$ among Black and $\mathrm{OR}=6.2$ among White elderly North Carolina smokers when compared to non smokers (Beck et al 1990). However, the microbial flora was also different among these groups and other potentially confounding factors were uncontrolled. In fact, those who smoked, had two specific types of bacteria present, visited the dentist infrequently and had few teeth, there was an $\mathrm{OR}=16.0$ compared to those who had none of the exposures. An increased $\mathrm{OR}=8.6(\mathrm{p}$ $<0.001$ ) was found among those with diabetes who smoked compared to diabetic nonsmokers. Two case control studies found that smokers had an OR of 2.5 (Bergstrom 1989) and 2.6 ( $\mathrm{p}<0.001$ ) (Haber and Kent 1992) compared to nonsmokers when age and sex-adjusted and plaque was controlled. Neither of these studies however obtained their controls from the same population as the cases. Any differences between groups on SES or other characteristics could have biased the results.

Specificity - Some studies suggest a direct influence of tobacco on the periodontium. Cross-sectional studies which control for plaque still found a difference in AP between smokers and nonsmokers (Sheihiam 1971; Bergstrom and Eliasson 1987). Rather than a specific influence of smoking on $\mathrm{AP}$, it may be that smoking and infrequent tooth cleaning behavior are examples of "risk-taking" behavior and related to individual psychosocial characteristics (MacGregor 1984). Smoking has not been shown to be significant in a regression analysis of edentulousness when other variables are included (Horning et al 1992). The association of smoking on edentulousness has received inadequate attention, particularly since edentulousness is frequently the result of periodontitis. 
Analogy - The adverse effects of smoking on general health and chronic illness have been well documented. Smoking has been shown to increase risk of lung cancer, cancer of the oral cavity, esophagus and larynx and to increase the prevalence of hypertension and cardiovascular diseases (Doll and Peto 1976; Ismail et al 1983; Abbott et al 1986) as well as to increase the prevalence of osteitis following oral surgery (Sweet and Butler 1979).

Coherence/Experiment - There is no evidence to support these criteria.

\subsection{CHRONIC DISEASES AND CONDITIONS}

\subsubsection{Diabetes}

Biologic Plausibility - Studies support the biologic plausibility of an association between diabetes and AP. Several comprehensive reviews of the literature suggest the biologic implications of diabetes on AP. For example: 1) As a result of diabetes, vascular changes occur in various organs and tissues, including the periodontium. Histological studies have shown a thickening of the blood vessels. A degenerative vascular change in gingival specimens has been observed. The width of the gingival capillary basement membrane among those with diabetes was four times larger than in those without diabetes. These changes in vascularity may influence delivery of nutrients/oxygen to the tissues; 2) Abnormal collagen metabolism affects collagen, the major protein of the periodontium. The integrity of collagen synthesis and its turnover are essential for periodontal health. Experimentally-induced diabetes has been shown to reduce collagen turnover and to induce collagenase activity of gingival tissue resulting in more rapid breakdown of periodontal connective tissue among those with diabetes; 3) Altered microbial flora appears to be present among those with diabetes; 4) Abnormal defense mechanisms related to humoral immunity 
or neutrophil function have been identified among those with diabetes (Ellenberger and Rifkin 1970; Manouchehr-Pour and Bissada 1983; Wilton et al 1988).

In a cross-sectional study of gingival blood vessels obtained from gingival biopsies, it was found that there was a marked increase in the thickness of the vessel walls and thickening of the basement membrane among those with diabetes compared to those without diabetes (Campbell 1971). Such thickening has been observed in vessels elsewhere in the body. It has been theorized that the result may be a reduction in flow of blood/oxygen/nutrients to the gingival tissues. The cross-sectional study design employed allowed only for association to be made, and temporality was not established. Additional limitations of the study were that the sample size of the study was limited to eight and age was not controlled.

In a quasi-experiment of leukocyte response following periodontal surgery in rats, leukocyte count decreased following surgery among the diabetic rats compared to non-diabetic rats. There was a less intense response of neutrophils one day post surgery which could have been related to impaired chemotactic activity/cell migration (Ramamurthy et al 1979). Similar results were observed in other animal models (Matsson et al 1980, Ramamurthy and Golub 1983). The extrapolation of these results to humans may be questioned.

Consistency - A recent comprehensive review of the literature concluded that AP is more prevalent/severe among those with insulin dependent diabetes mellitus (IDDM) and non-insulin dependent diabetes mellitus (NIDDM) (Manouchehr-Pour and Bissada 1983) than those without these conditions. Earlier studies are criticized for using inadequate/invalid measures of $\mathrm{AP}$ and diabetes. 
Animal studies have shown direct histologic effects of diabetes on the periodontium. It appears that there is no difference between diabetic/non diabetic rats in terms of $\mathrm{AP}$ when plaque is absent, but, in the presence of plaque, diabetic rats lose significantly more alveolar bone.

Epidemiologic data are inconsistent in their reported association between $\mathrm{AP}$ and diabetes due primarily to variations in study populations, methods and clinical measurements of both conditions. Most epidemiologic studies have been cross-sectional and unable to determine the temporal influence of diabetes on the development and progress of AP. Only one prospective study was reported. It showed an increased rate of CAL among women with diabetes compared to those without (Cohen et al 1970). The age range of the study population (18 - 35 years) was however narrow and young. Despite the fact that the study was limited to two years duration, differences were observed. The results may not be generalized to males since females have been shown to have better oral hygiene (Kelly and Van Kirk 1965; Miller et al 1987).

A number of cross-sectional studies have shown an association between AP and IDDM. AP is believed to be a complication of IDDM, with a higher prevalence of AP among those with diabetes (Cianciola et al 1982). A study of individuals with long-duration IDDM showed increased prevalence/severity of periodontitis (Hugson et al 1989). However, since both diabetes and AP are age-related, confounding must be considered. When age and plaque were controlled, the prevalence of AP was still greater among Veterans Administration (VA) patients with diabetes compared to those without diabetes (Belting et al 1964). Some young IDDM populations (10 - 18 years) have not shown an increased prevalence of periodontitis (Barnett et al 1984). However, it could be argued that the effects on 
periodontitis do not generally appear until $\geq 30$ years (Suzuki 1988) and would not have been evidenced in this restricted young age group.

A large cross-sectional study among the Pima Indians of Arizona, a population known to have an extremely high prevalence (50\% of $\geq 35$ year olds) of Type II, NIDDM, showed an increased OR $=2.81$ (95\% confidence interval 1.91 to 4.13) for AP among those with NIDDM when adjustments were made for plaque and demographics (Emrich et al 1993). There may have been an underestimate of the risk of AP since edentulous individuals were excluded from the study. The results of this study may not be generalizable to other racial and ethnic groups.

In a cross-sectional study of 624 elderly Ontario residents, diabetes was not found to be a risk indicator for AP (Locker and Leake 1993). However, the sample size was inadequate to include a sufficient number of individuals with diabetes, likely resulting in an inability to detect any association.

It was difficult to determine the consistency of the related literature because cross-sectional studies varied substantially in their methods of assessing not only AP but also diabetes. Measurement of AP and diabetic status are both subject to measurement error.

Biologic Gradient - The literature is inconclusive regarding any influence on AP of a biologic gradient of duration or level of metabolic control. Inconsistencies may be due to demographic differences in populations and methods but differences are probably most affected by measurement error for both conditions (Manouchehr-Pour and Bissada 1983). In some cases, cross-sectional studies have shown no relationship between periodontal status and glucose tolerance levels (Sastrowijota et al 1990). However, one study consisted of 135 young dental students in whom it could be argued, 
there is very little chance of finding variations in periodontal status (Suzuki 1988). The sample size might also have been inadequate to include a sufficient number of subjects with a family history of diabetes. Another cross-sectional study found no relationship between duration of diabetes/type of treatment and AP although AP was influenced by the plaque level (Nichols et al 1978). However, in a cross-sectional study of a subgroup of metabolically poorly controlled diabetics it was found that they had more gingivitis (Rvasti et al 1985) and AP (Tervonen and Knuuttila 1986) than those with moderate to good metabolic control. The difference remained after controlling for variation in plaque level.

Strength - A cross-sectional study of the prevalence/severity of NIDDM (type II) diabetes among the Pima Indians, a homogenous group in which the prevalence of NIDDM is high (50\% among $\geq 35$ year olds) found an increased risk of CAL $(\mathrm{OR}=3.43,95 \% \mathrm{CI}, 2.28$ to 5.16$) \quad(\mathrm{OR}=2.81$ when plaque and demographics were controlled) among those with diabetes. Measurement errors may be inherent in this type of study but it is not likely that there were systematic errors among either groups (Emrich et al 1993). The sample size $(n=1,342)$ was ample.

Specificity - Diabetes is not sufficient to cause AP in all individuals although it appears that the prevalence/severity of AP is greater among those with diabetes. The exact mechanism of action is not understood (Rosenthal et al 1988). A quasi-experimental laboratory study demonstrated that when nine individuals $(n=9)$ with diabetes were intensively treated with conventional insulin therapy, their long term metabolic control improved but there was no improvement over nine months in their periodontal health (Sastrowijota et al 1990); possibly due to their unchanged plaque levels and the short duration of the study. 
Coherence - Reviews of the literature describe several biologically plausible mechanisms of action of diabetes on AP, each of which coincides with the current concepts of the natural history of AP (Manouchehr-Pour and Bissada 1983).

Analogy - An individual with diabetes is believed to be predisposed to soft tissue and general infections due to compromised polymorphonuclear (PMN) chemotaxis and phagocytosis or humoral and cell mediated activity. Related microangiopathies may cause retinopathy. Diabetic complications of the kidney are also common (Rosenthal et al 1988). Cross-sectional studies have shown that diabetes is associated with an increased prevalence of cardiovascular disease (Zimmet 1992). However, others suggest that individuals with diabetes are not at increased risk for bacterial infections (Ellenberger and Rifkin 1970) particularly when metabolic control is good.

Experiment - A quasi-experiment of induced-diabetes in rats showed no difference in AP between diabetic rats and controls. However when plaque was introduced, the diabetic rats lost significantly more alveolar bone than those without diabetes (Manouchehr-Pour and Bissada 1983). Extrapolation of results to humans is questionable.

Temporality - There was no evidence to support this criterion.

\subsubsection{Hypertension and Ischemic Heart Disease}

Biologic Plausible - A review of the literature proposes that in AP, as in other chronic diseases, the role of the host defense mechanism should not be overshadowed by the role of the bacterial agent (Clarke and Carey 1985). 
The gingival margin is composed of small vessels where collateral circulation is minimal and there is a high nutritional demand to allow for quick collagen turnover rates. Diseases of the peripheral vessels may affect these end-organs and in turn, influence the surrounding tissues (Clarke and Carey 1985). Ischemic heart diseases which have been studied in relation to AP are acute myocardial infarction (MI), angina pectoris (angina) and coronary atherosclerosis (arteriosclerotic and atherosclerotic heart disease) International Classification of Diseases Codes: ICDC \#410, 413, and 414. (ICD.9.CM, 1979). Hypertension is rarely defined consistently. Hypertension as defined by the 1985 Canadian Blood Pressure Survey (Health and Welfare Canada 1989) and the 1989 Manitoba Heart Health Survey is: Current diastolic blood pressure $(\mathrm{DBP}) \geq 90 \mathrm{~mm} \mathrm{Hg}$ and/or on treatment (Young et al 1991) with medications.

The authors of a number of cross-sectional studies suggest biologically plausible explanations for an association with vascular disease and AP. In autopsy specimens, the mandibular arteries of patients with periodontitis were found to be nearly obliterated. The authors speculate that there was evidence of arterial disease in the periodontium and that periodontal health may have resulted from decreased circulation and influencing cell metabolism and defense mechanisms (Quintarelli 1957). However, the issue of temporality was not addressed and no account was made for age. Both factors challenge the study's validity, particularly since age is a correlate with both conditions. Another set of autopsy specimens of patients who had died of cardiovascular disease showed degenerative vascular changes in the oral mucosa (Stahl and Fox 1953). This study had no controls and did not account for any other potentially confounding patient characteristics. Others have shown that low SES, as measured by 
education, was associated with a higher cardiovascular risk profile, namely smoking, obesity and physical inactivity (Millar and Wigle 1986).

A histological study of periodontal vasculature among autopsy material from jaws of three individuals who died of arteriosclerosis also found vascular narrowing and calcification of the vessel walls in the periodontium (Grant and Bernick 1970). The findings do not prove that arteriopathies predispose the periodontium to periodontitis or that they preceded AP. However, they do show that the vessels of the periodontium are affected by arteriosclerosis. The effect, if any, on AP is unknown.

Two cross-sectional studies suggest an association between hypertension (high blood pressure) and AP based on the identification among hypertensives of an increased number of nutrient canals in the alveolar bone (Patel and Wuehrman 1976; Patni et al 1985). The studies did not control for age and were poorly designed. The suggested biologic plausibility that hypertension increases the number of nutrient canals by dilating the arterioles and/or by causing hyperplasia of the vessel resulting in new collateral vessel openings seems unfounded and is not supported elsewhere in the literature.

Very little attention has been given to the influence of vascular conditions on AP. However, theories suggesting an influence on AP by impaired peripheral vessels seem biologically plausible.

Consistency - Several cross-sectional studies have suggested an influence of vascular conditions on AP. In a vascular study among seven individuals with AP who had died from a variety of causes, histologic signs of arteriosclerosis were found not only in the aorta, intercostal, and renal arteries, but also in the mandibular artery. In addition, the vessel lumen were obliterated. The authors suggested an indirect relationship between 
cardiovascular disease and AP (Quintarelli 1957). However, the report did not indicate whether all individuals had vascular disease. There appeared to be no control specimens.

Other cross-sectional studies have found no increase in the prevalence of AP among 121 VA hospital patients who had cardiac disease. However, among 155 VA patients with arteriosclerosis, there was a "greater prevalence of periodontitis than expected" (Sandler and Stahl 1960). No statistical analysis was reported. The results may not be readily generalized because $90 \%$ of the subjects were males of low SES. Although inadequate statistical analyses were done, the results warrant further investigations.

A cross-sectional study of elderly Ontario residents found that general health conditions (including hypertension and heart disease) were not risk indicators for AP (Locker and Leake 1993). However, the sample size may have been inadequate to identify an association if it existed. A 14year longitudinal study of 20,000 subjects found dental disease was associated with an increased risk of coronary heart disease in young men (Destefano et al 1993). This association may have been due more to health compromising behavior than a direct influence of coronary heart disease and dental health. There was no account made for age, known to be correlated with both coronary heart disease and AP.

Two case-control studies assessed the association between dental health and acute myocardial infarction (MI). Dental health was found to be significantly worse among individuals with MI, even after adjusting for age, SES and smoking (Gilmour et al 1989; Matilla et al 1989). The validity of the study is challenged because the authors had to sample twice, using different inclusion criteria to obtain a sufficient number of controls. They also used a new, unvalidated aggregated dental index. 
There seems to be consistency of association between AP and vascular diseases/conditions among the few reported studies although the designs of most studies are somewhat suspect. Whether coronary heart disease is influenced by AP is uncertain although the plausibility has been suggested recently (Destefano et al 1993; Page 1995).

Specificity - A case history of gingival overgrowth associated with the administration of nifedipine showed that gingival overgrowth could be induced by the administration of nifedipine, and upon termination of administration, there was tissue resolution, with overgrowth returning upon subsequent re-administration (Lederman et al 1984). The periodontium appeared to be influenced more by the medication used to treat heart conditions (In this situation, nifedipine was used to treat angina pectoris.) than the heart condition itself. In a seven week quasi-experiment of hypertensive rats, obesity, not the animal's hypertensive state played a significant role in their development of periodontitis (Peristein and Bissada 1977). The limited studies available suggest that factors related to heart/ vascular conditions such as medications or obesity may be more directly influential in the development of AP than the vascular conditions themselves.

Analogy - Individuals with hypertension and coronary heart disease are more apt to have a higher prevalence of diabetes and other chronic health conditions (Department of Health and Human Services 1989).

Experiment - A seven-week quasi experiment in rats showed that hypertension alone was not a significant risk factor for periodontitis but that obese-hypertensive rats when compared to hypertensive or obese rats developed the most severe periodontitis (Peristein and Bissada 1977). 
Biologic Gradient / Temporality / Strength / Coherence - There was no evidence to support these criteria.

\subsection{MEDICATIONS WITH POTENTIAL DRUG ACTIONS}

Biologic Plausibility - Medications have been classified as to their effect on the oral cavity. Class I medications have a direct effect through mechanisms such as xerostomia or gingival overgrowth while class II medications may cause local or systemic complications for dental procedures. Class I medications are taken by $31 \%$ of $25-44$ year olds and $47 \%$ of $\geq 65$ years (Beck and Hunt 1985).

The relationship of sex hormone therapy to AP has been studied. A cross-sectional study of the effect of female sex hormones showed that pregnant women had more gingivitis than non-pregnant women with a correspondingly higher prevalence of Bacteroides bacterial species among those who were either pregnant or using oral contraceptives (OC's). The level of Bacteroides was 16x greater among OC users than non-pregnant women. Bacteroides species requires naphthaquinone, for which sex hormones may be a substitute (Jensen et al 1981). Several review articles propose mechanisms of action on AP by a number of other hormones. Plaque-induced gingivitis is accentuated by circulating levels of estrogens/ progesterone and androgens via partial immune suppression, increased fluid exudation, or stimulation of fibroblast activity. The main effects of these hormones/medications on the periodontium is gingivitis or pseudopocketing not CAL (Sooriyamoorthy and Gower 1989).

Nifedipine (Adalat) therapy has been shown to cause a higher prevalence of gingival overgrowth when compared to cardiac patients taking atenolol or a control. The mechanism is believed to be an effect of the 
medication on calcium transport (Barclay et al 1992). Another review article described the effect the antiepileptic, phenytoin (Dilantin) on the periodontium. It is thought that phenytoin has a direct effect on gingival fibroblasts (Seymour and Heasman 1988; Suzuki 1988). Medications causing gingival overgrowth, create a difficult-to-clean environment, potentially predisposing the individual to AP (Seymour and Heasman 1988).

Immunosuppressants are believed to act on the immune system, causing selective inhibition and suppression. Cyclosporin, like nifedipine and phenytoin, has been shown to produce gingival overgrowth, although gingivitis is less prevalent among those who take cyclosporin compared to controls (Seymour and Heasman 1988; Suzuki 1988).

The biologic plausibility of the relationship between the medications reviewed and gingivitis is reasonable however it may not apply directly to periodontitis. The importance of medication-induced gingival overgrowth in the initiation or progression of AP has not been adequately studied.

Consistency - A cross-sectional study found that women taking OC's for more than $1 / 2$ years, when compared to women using other forms of contraceptives, had lower plaque levels but higher levels of gingivitis and periodontitis (Knight and Wade 1973). The study population was restricted to 17 - 23 years olds. The population may have been too young to be able to detect differences in periodontitis, if it occurred. Small differences could be attributed to clinical measurement error. In addition, the study's duration was limited and recall bias may have occurred when subjects were asked whether there had been any breaks in their use of OC's over four years.

In another cross-sectional study, it was found that cardiac patients taking nifedipine had a higher prevalence/severity of gingival overgrowth than those taking atenolol or a control when plaque was controlled (Barclay 
et al 1992). This was a small $(n=19)$ sample size and causal inferences could not be made due to study design. Another cross-sectional study showed that patients who used cyclosporin and nifedipine had a higher prevalence of gingival overgrowth than those taking either medication alone (Thomason et al 1993). A significant correlation was found between overgrowth and plaque in both groups. The role of gingival overgrowth in the initiation of AP has not been clearly established. All evidence is crosssectional, therefore no causal inferences may be drawn.

Biologic Gradient - Cross-sectional studies reveal that current OC users had more gingivitis than those not taking OC's. There was however no relationship in severity/extent of gingivitis with different levels of estrogen/progesterone content among the different OC brands although some brands produced more gingivitis than others. There was also no influence on severity/extent of gingivitis with increased duration of OC use (Kalkwarf 1978). The examiners were blind to OC use, however, the data were subject to recall bias. In addition, the results apply to gingivitis, not to periodontitis. Although use of cyclosporin has been associated with gingival overgrowth and a dose-response relationship observed (Seymour and Heasman 1988), increased dosage of cyclosporin has not been associated with increased prevalence/severity of gingival overgrowth in another crosssectional study (Thomason et al 1993). There is conflicting evidence as to a dose-response relationship between phenytoin and gingival overgrowth (Seymour and Heasman 1988).

Specificity - A case history, earlier described, indicates the specificity of nifedipine to produce gingival overgrowth and for the condition to be reversed upon withdrawal of the medication (Lederman et al 1984). This 
overgrowth of tissue may foster periodontitis, but the medication itself does not cause AP.

Temporality / Strength / Coherence / Analogy / Experiment - There is no evidence to support these criteria.

\subsection{ORAL FINDING}

\subsubsection{Bacterial Dental Plaque}

Biological Plausibility - Bacterial plaque has been shown experimentally to be the primary etiologic agent of gingivitis ( Löe et al 1965; Suzuki 1988). It is believed that progression from plaque-associated gingivitis to periodontitis represents a conversion from an established to an advanced lesion (Page and Schroeder 1982; Suzuki 1988). This conversion may reflect aberrations of host cell responsiveness to plaque infection or may represent colonization and infection by highly pathogenic organisms (Suzuki 1988). Three biologically plausible hypotheses of causality have been proposed: 1 ) direct tissue destruction caused by plaque and its toxins; 2) immune hyperresponsiveness; or 3) immune deficiencies (Suzuki 1988). In support of these explanations, it has been demonstrated that certain bacterial species invade the periodontium. While this invasion corresponds to episodes of tissue breakdown, (Newman 1990) there is no evidence to prove that it causes the destruction. Some studies report that patients with advanced periodontitis have a depressed lymphocyte blastogenic response to plaque, while others find an acceptable range of responsiveness once healthy subjects were age and sex-matched (Suzuki 1988).

Degradation of gingival tissues may be caused by pathologic alternations of fibroblasts, the influx and activation of PMN's/macrophages and their release of collagenase and other connective tissue degrading 
enzymes. Bone resorption is believed to result from processes involving products of activated lymphocytes, prostaglandins, and perhaps direct effects of bacterial substances. All of these processes have been shown to be activated experimentally by subgingival bacteria (Page and Schroeder 1982) in vitro or in animal models. The ecological theory of causation suggests that shifts in the proportions of bacterial species in plaque result in imbalances of some species and the development of periodontitis (Newman 1990). Since no single species has been shown to cause AP, the theory is plausible. However, bacterial species have been shown to differ between gingival health, and various stages of gingivitis with predominantly gram positive cocci and rods in health changing to filamentous flora, vibrious and spirochetes in more established lesions. In a $11 / 2$ year quasiexperiment, dogs were assigned to test or control groups to study the influence of plaque accumulation on the development of periodontitis. In the presence of plaque, periodontitis developed while plaque-free teeth showed no signs of gingivitis or periodontitis (Weintraub and Burt 1985). The generalizability of findings to humans may be questioned.

Consistency - Numerous cross-sectional studies employing various methodologies have shown consistently that poor plaque control is associated with an increased prevalence and severity of periodontitis among a wide variety of populations (Kelly and Van Kirk 1965; Kelly and Harvey 1979; Burt et al 1982; Abdellatif and Burt 1987; Brown et al 1990; Beck et al 1990; Beck et al 1992). However, since AP and plaque were assessed concurrently, temporality was not established.

Clinical trials have also supported plaque as a causative agent (Axelsson and Lindhe 1978; Page and Schroeder 1982). A number of defined bacterial species have been associated with periodontitis (Suzuki 1988; Beck 
et al 1992; Haffajee et al 1993). Experimental animal (Lindhe et al 1973) and human (Axelsson and Lindhe 1978) studies support plaque as a causative agent. Experimental gingivitis in humans is known to be caused directly by bacterial plaque ( Löe et al 1965; Page 1986). Although the presence of plaque has been shown to be a strong clinical predictor of future attachment loss in a one year longitudinal study in humans (Haffajee et al 1993), the association of plaque with progressive attachment loss may have been due to plaque's association with attachment loss at baseline. Few studies have failed to find an association between plaque and periodontitis (Africa et al 1985; Baelum et al 1986; Löe et al 1986; Griffiths et al 1988). Some individuals/populations appear to be resistant to bacterial plaque. Evidence from several longitudinal studies suggest that plaque is a necessary but not sufficient cause of periodontitis (Curtress et al 1982; Africa et al 1985; Baelum et al 1986; Reddy et al 1986).

Biologic Gradient - Cross-sectional and clinical studies have shown that with increasing plaque, there is an increase in the prevalence of periodontitis (Kelly and Van Kirk 1965; Burt et al 1982; Miller et al 1987; Abdellatif and Burt 1987; Brown et al 1990; Machtei et al 1992; Haffajee et al 1993). Few studies failed to show evidence of a differential response to increasing gradients of plaque (Baelum et al 1986). Most studies did not measure plaque mass or thickness directly but rather determined the proportion of teeth with plaque. Although perhaps not directly applicable to periodontitis, the initiation of experimental gingivitis is preceded by a gradual increase in mass and thickness of plaque (Löe et al 1965; Lindhe et al 1973; Caton 1993) and related changes in the bacterial composition which occur with increasing thickness of plaque. Whether it is changes in mass or bacterial composition which influences the conversion of gingivitis to 
periodontitis is uncertain. However, it has been proposed that increasing thickness of plaque may alter the ecology of plaque, resulting in more gram-negative anaerobic bacteria, known to be associated with the initiation of periodontitis (Page and Schroeder 1982). It may be, however, that periodontitis itself is responsible for some of these ecological shifts in bacterial species.

Temporality - The initial and early gingivitis lesion may be induced experimentally by applying extracts of plaque-bacteria to healthy gingiva of animals (Page 1986). The classic experiment of Löe et al demonstrates that when plaque is allowed to accumulate for 21 days in periodontally healthy individuals, gingivitis is induced and subsequent plaque removal results in resolution of gingivitis (Löe et al 1965; Page 1986). Löe's experiment satisfies the temporal criteria in the cause of gingivitis. However, periodontitis was not investigated.

A well-controlled quasi-experiment among dogs satisfied the temporal criteria for causality of periodontitis. Periodontitis was induced among initially healthy dogs by allowing plaque to accumulate on test teeth for 18 months (Lindhe et al 1973). The generalizability of these results to humans is uncertain. A three year quasi-experiment demonstrated that periodontitis could be prevented in adults maintained at a high standard of oral hygiene compared to a control group undergoing standard dental care (Axelsson and Lindhe 1978) i.e., less rigorous attention to frequent plaque removal. Although there were no differences between test and control groups at baseline, the study's internal validity was threatened by failure to randomize assignments.

Strength - A recent re-analysis of secondary epidemiologic data taken from cross-sectional studies suggested a strong relationship between 
periodontitis and oral hygiene, the odds ratio $(\mathrm{OR})=20.52$ (95\% confidence interval 17.75 - 23.72) (Abdellatif and Burt 1987). The incidence rates for periodontitis in all ages were higher among those with poor oral hygiene. The etiologic fraction indicated that at least $88 \%$ of periodontitis among all exposed groups could be attributed to oral hygiene. The validity of the statistical methods used to estimate incidence from age-specific prevalence among the 15,000 dentate Americans may be challenged because prevalence is a function of both incidence and duration. However, since periodontitis is generally an irreversible, non-fatal condition, incidence could reasonably be estimated from age-specific prevalence.

Other cross-sectional studies have assessed the strength of association between plaque and periodontitis and have concluded that among Black elderly North Carolina residents, the OR for periodontitis for those with B. gingivalis above $2 \%$ was 2.4 and for B. intermedius, 1.9 (Beck et al 1990,1992). Others report an increased $\mathrm{OR}=2.3$ among those with poor oral hygiene practices (Locker and Leake 1993). However when other variables were controlled, this finding often disappeared. Many of these cross-sectional studies have found increased odds of periodontitis associated solely with plaque, but also with other variables suggesting a complex interactive and multifactorial relationship. The few studies which failed to show a significant relationship between plaque and periodontitis were among unique populations/individuals who appeared to be periodontitis-resistant (Africa et al 1985; Löe et al 1986; Baelum et al 1986). Only recently have measures of magnitude been applied to study periodontitis but those which exist tend to support the association.

Specificity - Certain bacteria have been shown to be explanatory variables in predicting periodontitis (Beck et al 1990; Haffajee et al 1993). Studies have 
revealed varying degrees of specificity of particular species of bacteria in association with health/ gingivitis/ periodontitis (Caton 1993). There is little doubt that periodontal diseases are infections, many being caused by specific organisms. In fact, specific species or combinations of species may be associated with different diseases (Suzuki 1988). Yet, no specific bacterium has been shown to be causative for AP (Newman 1990; Ismail and Lewis 1993). Studies are hampered in identifying specific bacteria due to methodological sampling/ culturing problems.

The specificity of plaque in gingivitis and periodontitis was described earlier (Löe et al 1965; Lindhe et al 1973). The presence of plaque is not always sufficient to cause periodontitis as evidenced in longitudinal and cross-sectional studies among periodontitis-resistant populations/ individuals (Page and Schroeder 1982; Cutress et al 1982; Africa et al 1985; Baelum et al 1986; Löe et al 1986).

Coherence - The relationship between plaque and periodontitis does not conflict with the current understanding of the natural history of the disease. Studies which suggest plaque as a predictor of future destruction coincides with our understanding of the influence on the periodontium of extended plaque exposure (Löe et al 1965; Lindhe et al 1973; Haffajee et al 1993) and the natural history of disease (Page and Schroeder 1982) as well as with tissue response to alterations in the ecology of the microbial flora (Newman 1990). That periodontitis can be prevented by rigorous plaque control (Axelsson and Lindhe 1978) is also in keeping with Löe's classic experimental gingivitis studies (Löe et al 1965) and the natural progression of the disease.

Analogy - Dental caries (decay) results from the action of bacterial metabolic end products localized on the tooth surface in dental plaque 
(Nikiforuk 1985). It has been shown that both caries and AP could be prevented/controlled when plaque was rigorously eliminated (Axelsson and Lindhe 1978). However, the generalizability/feasibility of a program of twice monthly professional prophylaxis is questionable.

Experiment - Two experiments described previously (Löe et al 1965; Lindhe et al 1973) confirmed the etiologic role of plaque in periodontitis in animal models and gingivitis in man. In general, most plaque-related studies have been cross-sectional in nature. Many have excluded edentulous individuals or other highly susceptible groups such as the unemployed (Brown et al 1990) which could have underestimated association.

Some studies looked at isolated risk variables, failing to consider the multifactorial nature of periodontitis or the importance of controlling for confounders. Cross-sectional studies are unable to address temporality of the associations or the specificity of bacterial species since prevalence and exposure are assessed concurrently. As a result, inference may not be drawn from such studies. Since randomization was not employed in a number of quasi-experiments, validity is suspect. These alternate research designs were most likely chosen because ethical considerations prohibit experimental inducement in man of a potentially irreversible condition such as periodontitis.

Only a few studies have failed to find an association between plaque and periodontitis. These results however may be biased due to inaccurate self-reports of brushing frequency or to the transient nature of plaque or to the Hawthorne effect. The inter/intra - rater reliability of the plaque indices and CAL was often unreported or low. Poor reliability could have resulted in a failure to find an association if it existed. A number of studies which 
failed to find an association were conducted in apparently periodontitisresistant populations or under compromised field settings (Kingman et al 1991).

Table 4.1 - STRENGTH OF SCIENTIFIC EVIDENCE

Sociodemographic Variables

\begin{tabular}{|c|c|c|c|c|c|}
\hline CRITERIA & $\overline{A G E}$ & GENDER & EDUCATION & RACE & INCOME \\
\hline $\begin{array}{l}\text { Biological } \\
\text { Plausibility }\end{array}$ & 292 & & & & \\
\hline Consistency & $\begin{array}{l}292,185,17 \\
294,186,219 \\
161,192,143, \\
45,202,-112, \\
-52,-122, \\
-146,-3\end{array}$ & $\begin{array}{l}16,180,137 \\
161,192,143 \\
45,202,223 \\
-122,-112\end{array}$ & $\begin{array}{l}60,284,274, \\
185,161,45 \\
202,180,18 \\
-112\end{array}$ & $\begin{array}{l}202,137,195 \\
45,27,-161 \\
-112,-274,-26\end{array}$ & $\begin{array}{l}60,207, \quad 12, \\
161,202,45 \\
21\end{array}$ \\
\hline Biologic Gradient & $\begin{array}{l}292,17,294 \\
186\end{array}$ & & & & \\
\hline $\begin{array}{l}\text { Temporality } \\
\text { Strength }\end{array}$ & -3 & 24 & & & \\
\hline Specificity & $-294,-52,-88$ & $\begin{array}{l}-192, \quad-16 \\
-180,-41\end{array}$ & $\begin{array}{l}-180,-41,-68 \\
-302\end{array}$ & $-41,-274,-26$ & $\begin{array}{l}-41,-68,-207 \\
-21,-47\end{array}$ \\
\hline Coherence & $\begin{array}{l}17,292,186 \\
219,-122\end{array}$ & & & & \\
\hline $\begin{array}{l}\text { Analogy } \\
\text { Experiment } \\
\text { Highly Convincing } \\
\text { Conflicting } \\
\text { Evidence } \\
\text { Not Convincing } \\
\end{array}$ & $\sqrt{ }$ & $\sqrt{ }$ & $\sqrt{ }$ & $\sqrt{ }$ & 21 \\
\hline
\end{tabular}

Health Compromising Behavior

\begin{tabular}{ll}
\hline \multicolumn{1}{c}{ CRITERIA } & \multicolumn{1}{c}{ SMOKING } \\
\hline Biological Plausibility & $38,243,64,75,163,83,168,31,72,251$ \\
Consistency & $27,174,26,185,137,35,109,34,237,37,117,147,236,266,33,233$, \\
& $37,-147$ \\
Biologic Gradient & \\
$\begin{array}{l}\text { Temporality } \\
\text { Strength }\end{array}$ & $27,26,35,117$ \\
$\begin{array}{l}\text { Specificity } \\
\text { Coherence }\end{array}$ & $33,-137,-266,-191$ \\
$\begin{array}{l}\text { Analogy } \\
\text { Experiment } \\
\text { Highly Convincing } \\
\text { Conflicting Evidence } \\
\text { Not Convincing }\end{array}$ & $147,82,2,283$ \\
\hline
\end{tabular}


Chronic Diseases, Conditions \& Medications

\begin{tabular}{|c|c|c|c|}
\hline CRITERIA & DIABETES & $\begin{array}{c}\text { HYPERTENSION \& } \\
\text { ISCHEMIC HEART } \\
\text { DISEASE } \\
\end{array}$ & MEDS \\
\hline Biological Plausibility & $\begin{array}{l}196,248,303,84 \\
56,241,199,240\end{array}$ & $\begin{array}{l}238,277,110,221 \\
63\end{array}$ & $22,153,275,19,263$ \\
\hline Consistency & $\begin{array}{l}196,139,85, \quad 286 \\
69,62,29,-203,-185\end{array}$ & $238,198,-185,-224$ & $\begin{array}{l}22,19,263,288 \\
282,167\end{array}$ \\
\hline $\begin{array}{l}\text { Biologic Gradient } \\
\text { Temporality } \\
\text { Strength }\end{array}$ & $\begin{array}{l}286,252,-196,-259 \\
-206 \\
85\end{array}$ & & $-288,-263,-157$ \\
\hline Specificity & $-248,-258$ & $-244,-173$ & 173 \\
\hline Coherence & 196 & & \\
\hline Analogy & $248,305,-84$ & 78 & \\
\hline $\begin{array}{l}\text { Experiment } \\
\text { Highly Convincing } \\
\text { Conflicting Evidence } \\
\text { Not Convincing }\end{array}$ & 196,199 & -244 & $\begin{array}{c}\sqrt{ } \\
\text { Gingivitis not Periodontitis }\end{array}$ \\
\hline
\end{tabular}

\section{Oral Finding}

\begin{tabular}{|c|c|}
\hline CRITERIA & PLAQUE \\
\hline Biological Plausibility & $218,189,205,282,176,58$ \\
\hline Consistency & $\begin{array}{l}218,176,27,26,192,122,13,53,45,160,161,202,3,-8,-244,-74,-115 \\
-16,-186\end{array}$ \\
\hline Biologic Gradient & $189,176,58,192,53,45,202,3,34,125,146,-27,-8$ \\
\hline Temporality & $189,176,13,215$ \\
\hline Strength & $26,3,27,-27,-8,-186,-185$ \\
\hline Specificity & $189,282,176,58,27,26,34,-8,-244,-74,-186,-146,-27,-218$ \\
\hline Coherence & $218,189,205,176,58,34,215,26,-27,-8,-74,-186$ \\
\hline Analogy & $189,215,26$ \\
\hline $\begin{array}{l}\text { Experiment } \\
\text { Highly Convincing } \\
\text { Conflicting Evidence } \\
\text { Not Convincing } \\
\end{array}$ & 189,176 \\
\hline
\end{tabular}

Support or lack of support (-) for each variable according to Hill's Criteria. Numbers represent references in bibliography. 


\subsection{CONCLUSIONS}

Bradford Hill's Criteria for Causation constituted the theoretical framework employed to assess the strengths and weaknesses of the scientific evidence to support an association between key variables and adult periodontitis. As evidenced in Table 4.1, there is highly convincing evidence that both bacterial plaque and cigarette smoking have a causal relationship with AP. Documented evidence of diabetes as an independent risk factor for $\mathrm{AP}$ is less convincing. Although age, gender, education, income, and race may be correlates with or determinants for AP, the literature does not suggest they play a causal role.

Use of specific medications may cause gingival conditions. However, a causal inference with periodontitis may not be drawn.

There is inadequate evidence to support or negate a causal role in AP for hypertension or ischemic heart disease.

Further cross-sectional and case-control studies are warranted to clarify the strength of association between AP and hypertension/ischemic heart disease, diabetes, and certain medications. Experimental or longitudinal prospective studies should focus on confirming the role of cigarette smoking and/or plaque as risk factors for AP while considering the multifactorial nature of the disease. 


\section{CHAPTER FIVE \\ DESIGN OPTIONS FOR RISK ASSESSMENT}

\subsection{INTRODUCTION}

In this chapter, various study design options were reviewed and their advantages and disadvantages weighed in light of the purposes of the current study. A summary of these deliberations follows.

There are three broad categories of empirical research: Experiments, which employ randomization of factors of interest to the subjects; Quasiexperiments, in which there is no randomization but the investigator artificially manipulates the study factor; and Observational studies, in which there is no manipulation by the investigator of the study factor (Kleinbaum et al 1982).

\subsection{EXPERIMENTAL versUS OBSERVATIONAL STUDIES}

Experimental studies are best able to control for extraneous variables and quantitative or temporal features of the study (Schlesselman 1982; Kleinbaum et al 1982). Quasi-experiments have less control over extraneous variables but they also have fewer practical hurdles than experiments. Experiments permit a direct means of inferring that an association between two factors is causal. However, experiments are often lengthy, due to long latency periods between exposure and outcome and they typically require large numbers of subjects. In addition, ethical considerations would not permit imposition of risk factors in human experimentation (Schlesselman 1982). Community intervention random controlled trials (RCT's) may however be utilized to reduce the risk factor of interest in an experimental group in order to establish disease causality (Bader 1990). 
However such studies are dependent on the ability of the intervention to reduce the risk behavior.

Observational studies can estimate disease frequency and formulate specific hypotheses. Analytically-based observational studies often follow descriptive studies, their purpose to identify potential risk factors, estimate their effect and to suggest potential interventions (Kleinbaum et al 1982). Both observational and experimental studies are able to clarify cause-andeffect relationships (Schlesselman 1982).

Observational research is often less expensive/time-consuming than experiments. Such studies usually take place in natural settings making the study population more representative of the target population. It is frequently a practical, feasible design since the investigator does not manipulate the risk factor(s) under study (Kleinbaum et al 1982). However, since there is limited control over the study variables, potential sources of error may threaten the internal validity of the study. These studies are difficult to replicate, threatening their external validity (Kleinbaum et al 1982).

\subsection{ANALYTIC DESIGNS}

\subsubsection{Cross-Sectional Studies}

Cross-sectional studies are observational studies which assess exposure and disease status simultaneously. They are useful for determining prevalence of conditions in a population, e.g. periodontitis; the distribution of particular characteristics, e.g. age; or the frequency of health compromising behaviors, e.g. smoking. However, since exposure and outcome are assessed concurrently, it is not possible to establish temporality. In addition, since cross-sectional surveys consider prevalent cases, they reflect determinants of survival as well as etiology. Despite their 
shortcomings, the results of such studies are often the first step in the formulation of etiologic hypotheses. Most evidence of potential risk factors, indicators and determinants of periodontitis has been obtained from crosssectional observational studies (Miller et al 1987; Brown et al 1990; Beck et al 1992; Locker and Leake 1993) This design cannot directly test etiologic hypotheses (Kleinbaum et al 1982; Hennekens and Buring 1987).

\subsubsection{Cohort Studies}

Another type of analytic observational study is the cohort study. It proceeds from a postulated cause to effect, similar to an experiment. Cohorts are observed over a specific period of time, after having been classified on the basis of certain characteristics. Groups are subsequently compared with regard to the outcome of interest (Schlesselman 1982). Cohort or follow-up studies may be used in risk factor studies in which a group (cohort) of individuals are identified, based on the presence/absences of exposure to a suspected risk factor. Individuals must be apparently free of the disease under investigation at the beginning of the study and subsequently followed over a specific (often lengthy) time to determine the occurrence, i.e., incidence of the disease (MacMahon and Pugh 1970; Hennekens and Buring 1987).

Current cohort studies are those in which the outcome occurs after the investigation begins. In historical cohort studies, on the other hand, the outcome has already taken place before the study is initiated. Cohorts are determined in the latter situation through data from existing records (MacMahon and Pugh 1970; Schlesselman 1982). A prospective study permits direct estimation of incidence rates for those with and without a particular attribute. The ratio of these rates is the relative risk. They can establish temporal sequence and evaluate multiple effects from a single 
exposure. Since the disease has not occurred at the start of a prospective cohort study, bias in subject selection and establishment of exposure is minimized (Hennekens and Buring 1987). Retrospective cohort studies usually are conducted more quickly and inexpensively than prospective cohort designs. They are efficient for a cohort investigation of conditions with a long latency period but depend on availability of accurate preexisting records and thus are subject to information bias. In addition, information on potential confounding variables may not be available from existing records.

Cohort studies are not exempt from biases. Selection bias may occur if both groups are not susceptible to the outcome of interest. Information and measurement bias may occur when determining the exposure status. Subjects may also change their exposure status. Failure to establish diagnostic criteria prior to study initiation or failure to measure both groups uniformly for the presence of disease may result in bias. External validity of cohort studies may be influenced by non-participation, withdrawals, and losses to follow-up. The proportion of subjects lost must not differ between exposure groups and the number of subjects lost to follow up must be minimized (MacMahon and Pugh 1970; Kelsey et al 1986). Limitations of the cohort design include: 1) If prospective, they may be expensive and time consuming since they follow large cohorts over lengthy periods of time. 2) There is the potential for bias and reduction of external validity due to losses to follow-up. 3) They are inefficient for the evaluation of rare diseases, unless attributable-risk is high (Hennekens and Buring 1987). 


\subsubsection{Case Control Studies}

Another type of analytic observational study is the case-control (C-C) or retrospective study which proceeds from effect to cause, attempting to identify antecedents that led to the disease. The use of control groups to infer causality came from the experimental methods formalized in the 18th and 19th centuries (Sartwell 1974; Schlesselman 1982). The C-C design is based on the selection of subjects with a condition (cases) and a comparison group(s) of individuals in whom the condition is absent (controls). Cases and controls are compared in terms of their exposure to variables under study (Schlesselman 1982; Hennekens and Buring 1987). For example, the prevalence of periodontitis among Pima Indians with NIDDM diabetes has been compared to Pima Indians without diabetes. Results indicated that those with diabetes were three times more likely to have moderate to severe periodontitis (Emrich et al 1993). Case-control studies may be exploratory whereby multiple hypotheses are proposed to gain enough knowledge about the disease and potential risk factors to subsequently form a specific etiologic hypothesis. However, case-control studies which are designed to test specific hypotheses have more scientific credibility (Schlesselman 1982).

There are several advantages to the case-control design: 1) When studying diseases with long latency periods such as chronic periodontitis, it is possible to identify a sufficient number of cases immediately and not wait years for the disease to develop. 2) The design permits identification of adequate numbers of cases of diseases which may be somewhat rare. 3) The association between a disease and a wide range of potential etiologic exposures can be evaluated with the C-C design and as such, the design is useful in hypothesis development. 4) C-C studies may be relatively quick and inexpensive. 5) Existing records may be utilized. 6) There is no imposed risk to subjects (Schlesselman 1982; Hennekens and Buring 1987). 
The limitations of the case-control study design are: 1) There may be difficulty in establishing antecedent exposure to the "risk factor" prior to the development of the disease. 2) C-C studies are particularly susceptible to certain biases as described in Section 5.3 (Sartwell 1974; Sackett 1979). 3) C$\mathrm{C}$ studies are inefficient for evaluating rare exposures. 4) Incidence rates can not be computed directly, unless the study is population-based (Hennekens and Buring 1987). This may not be a serious limitation since absolute risk is generally known from other available epidemiological data on similar populations (Sartwell 1974). 5) Validation of data may be difficult to carry out. 6) Control of extraneous and confounding variables may be incomplete. 7) Detailed study of the mechanism of causation is usually not feasible. 8) The C-C study is not suited for evaluation of therapy or for diseases of high incidence and short duration such as influenza (Sartwell 1974; Schlesselman 1982; Hennekens and Buring 1987).

\section{$5.3 \quad$ STUDY BIAS}

Biases that may affect case-control studies are discussed along with methods to evaluate and control them (Schlesselman 1982) including selection bias. Case selection bias may occur if study volunteers are different from those who do not volunteer regarding the exposure and outcome under investigation (Hennekens and Buring 1987). Participants should be compared to non-participants regarding key variables. Sources of error may also come from selection of controls. Ideally, controls should come from the same population as cases and have similarity in the attributes of interest (Mantel and Haenszel 1959; Sartwell 1974).

Surveillance bias may occur in a case-control study when cases are more likely to be detected among those seeking medical/dental care than those who do not. It has been suggested that there may be danger in 
making inferences or generalizations from a hospital-based study because diseases have different probabilities of leading to care. As a result, spurious disease associations could be made in such settings. Where the results are not generalized beyond the target population of hospital (clinic) patients, the issue is of little relevance (Sartwell 1974).

Therapeutic intervention bias may occur when cases are treated differentially from controls during the study. It is important that the investigator be blind to the subjects' outcome status during the study. Diagnostic suspicion bias may result from the investigator's prior knowledge of the subject's exposure status influencing the diagnostic process. Where antecedent exposure to risk factors is determined subsequent to case diagnosis, there is no concern. Non-respondent bias may occur when one group of individuals responds to a survey/participates differently from the other group. For example, smokers may be less likely to participate in telephone health history interviews. To protect against this bias, the investigator should attempt to achieve at least an $80 \%$ response rate. Although there is no reason to believe that there would be a difference between response rates among smokers who are cases vs smokers who are controls, it is important to compare them (Sackett 1979).

Observation bias may result from errors in obtaining information from study subjects. The subject's knowledge of his/her disease status can influence how (s)he reports information. However, if the subject is unaware of the purpose of the study and attention is not drawn to their periodontal status, it is not likely that their responses would be influenced. The examiner may record interview data with bias if he is aware of the exposure or disease status of the subject (Shortell and Richardson 1978). Interviewer bias may also occur if differences in interview intensity exist between cases and controls. Blinding interviewers to outcome status 
reduces this problem. Measurement and information bias are reduced in retrospective studies by using a structured, pre-tested questionnaire and blind, calibrated, and trained interviewers (Sartwell 1974).

Recall bias may occur if the recall of cases vs controls differs in amount or accuracy. There is nothing about having periodontitis that would prompt cases to recall their exposures differently from controls. Indeed, many cases/controls will be unaware of their periodontal status or make any association with it and the exposure of interest (Sackett 1979).

Accuracy of medication histories obtained through retrospective studies may suffer from problems related to recall. However, several retrospective and cohort studies have found that there was strong agreement between prescriber records and patient reports on information such as: whether oral contraceptive or climacteric estrogen had ever been used (89\% agreement), the name of the medication (85\%), dosage (88\%), and total duration of drug intake (85\%) (Glass et al 1974; Persson et al 1987). Others have found that the name of the most recent oral contraceptives was remembered adequately (89\%) but that the name of previous oral contraceptives was less well recalled (63\%) (Stolley et al 1978). Studies have found a deterioration of recall with time (Glass et al 1974; Stolley et al 1978; Persson et al 1987). Some studies were limited by small sample size, others by including only women of high SES who were strongly motivated toward family planning. These flaws challenge the generalizability of the results to other populations or to other medications (Glass et al 1974). Most studies dealt with a relatively short duration of recall which is of concern when attempting to apply the results to studies of life-time exposure to medications.

Although complete and accurate recollection and reporting of medications appears to diminish with time and lessening degrees of 
salience (Norman 1986), bias due to recall is minimized where the medications of interest are of a salient nature, i.e., medications for significant health conditions such as diabetes and heart disease. In addition, bias is not likely since there is no reason to believe recall of medications will differ between cases and controls.

To address recall bias, the investigator could obtain supplementary information regarding antecedent exposures using alternate methods. For example, in addition to an interview, pre-existing medical records could be reviewed to determine exposure. Since these records were obtained prior to patient diagnosis, they could not have been influenced by the outcome status (Hennekens and Buring 1987). Recall bias may occur when medical records or interviews used in case-control studies are unreliable/invalid due to memory loss, confusion or effort to please interviewer. Where variables of interest are clear and common (e.g. history of ever smoking) or when an exposure is significant (e.g. diabetes) there is less concern for memory bias. Telephone interviews are often less affected by socially sensitive questions than in-person interview (Schlesselman 1982; Kidder and Judd 1986).

Recall bias may affect the study's ability to detect underlying associations particularly when there is a difference between cases and controls regarding their prospensity of recall bias. If one group's memory is better than the other's or if one group is less likely to respond or respond correctly to questions on an interview, the internal validity of the study is threatened. There is no reason to believe that cases would behave any differently than controls in this matter.

Recall bias may be assessed by re-testing a random sample of subjects within two weeks to determine whether respondents provide the same answers to the questions at both interviews. This procedure measures 
consistency of recall and reliability of the questionnaire and interviewers. (Schlesselman 1982).

Validity of the interview may be tested by obtaining confirmatory information regarding exposures from supplemental sources such as the dental chart and comparing this to the telephone interview responses (Schlesselman 1982).

Prevarication bias may occur if subjects have ulterior motives for over/underestimating exposures. There is no reason to believe that cases and controls would differ in this respect. Another type of bias is misclassification bias in which errors are made in the categorization of exposure status. Random misclassification is difficult to avoid and it results in an underestimate of effect. However, non-random misclassifications threatens internal validity and must be avoided. Accuracy or completeness of information must not differ between cases and controls as it could result in an underestimate or exaggeration of effect (Hennekens and Buring 1987). Misclassification bias may also occur if a subject's disease status is subject to error. Odds ratio estimates will be biased as a result of misclassification. They may either over or underestimate the association between exposure and disease. Measurement error may be minimized by restricting cases and controls to those individuals who have undergone identical diagnostic examinations using the same clinical tests and by using the most critical diagnostic criterion (Schlesselman 1982).

Other Biases - Inadequate sample size is another source of error which biases the study toward accepting the null hypothesis of no association (Schlesselman 1982). Ensuring that the study has ample subjects and a high response rate remedies this problem. Consideration also must be given to the role of confounding (Hennekens and Buring 1987). 
Confounding variables can cause or prevent the outcome of interest thus distorting the effect of the variable under investigation (Last 1988). Confounding can be controlled in a number of ways. One way is to select controls who have the same characteristics as the cases, except for the exposure under study. For example, since periodontitis is highly correlated with age (Burt 1994), matching or stratifying cases and controls by age accounts for the confounding effect of age. Unfortunately, once "matched" on age, the study can not examine the effect of age on the disease. Multiple regression is a statistical method used to control for confounders such as age (Sartwell 1974; Rothman 1986; Kelsey et al 1986).

In retrospective studies of multiple risk factors, the potential number of associations which may arise are numerous. There is a good chance that when applied to many associations, statistical tests will produce a type I error, i.e., finding an association when none actually exists. Conservative levels of significance testing should be considered. However, since the retrospective study is looking for leads, not proof, and since the credibility of the results of such observational studies are assessed for their plausibility/consistency with other sources of evidence, there is less concern over multiple significance testing (Mantel and Haenszel 1959).

\subsection{CONCLUSIONS}

While most authors recognize that there are strengths and limitations inherent in both case-control and cohort study designs (Mantel and Haenszel 1959; Sartwell 1974; Rothman 1986; Hennekens and Buring 1987), most suggest that, in the absence of an experiment, a well-designed cohort study is an extremely valuable strategy to obtain a valid estimate of the association between an exposure and disease (Sackett 1979; Hennekens and Buring 1987). It must be recognized however that prospective cohort 
studies must also deal with challenges of bias, confounding, and appropriate controls. Although often stressed that "retrospective studies cannot prove causation" the implication that observational cohort studies can do so is false. Such proof is only possible through well-designed experimental studies (Mantel and Haenszel 1959; Sartwell 1974; Sackett 1979; Hennekens and Buring 1987).

The ultimate choice of study design for the current study was "case control" based on: 1) the hypotheses being tested; 2) the resources available; and 3) the current state of knowledge. The case-control method allowed a sufficient number of patients with periodontitis to be identified readily. It allowed testing of multiple risk indicators, markers, and determinants. Since there is little scientific evidence to support several of the variables of interest (e.g. ischemic heart disease), a hypothesis-forming study is appropriate. The selected design also permitted use of available secondary data from clinical records while incorporating an additional methodology, telephone interviews.

The limitations of the case-control design are recognized. The fundamental limitation is that causality may not be proven since temporality can not be firmly established. 


\section{CHAPTER SIX}

\section{STUDY DESIGN}

\subsection{INTRODUCTION}

A case-control study incorporating a blind telephone-interview was designed to identify individuals with a history of moderate to advanced periodontitis (cases) and those without (controls) and to compare each group's exposure to a number of variables including: health compromising/enhancing behaviors, history of chronic diseases/ conditions, use of specific prescription medications, sociodemographic variables, as well as oral/ dental findings.

\subsection{STUDY SETTING AND TARGET POPULATION}

The University of Manitoba, Faculty of Dentistry (FOD) operates a teaching-clinic at the Bannatyne Campus, located at the Health Sciences Centre in downtown Winnipeg, Manitoba, Canada. The primary function of the dental clinic is to provide a facility for clinical teaching opportunities and experience for undergraduate students in dentistry and dental hygiene. Undergraduate students provide community dental services to the general public. Fees for dental care during the study period were approximately one-third of the Manitoba Dental Association's Recommended Fee Guide (Cleghorn 1994). The demographic profile of the patients attending the clinic is uncertain. It is believed that the unemployed, retired and/or those from lower socioeconomic groups may be attracted to the FOD because of reduced fees.

The target population of the current study was identified from patients who sought dental care at the FOD clinic over the period January 1991 - June 1994. 
Patients who sought care at the clinic received a cursory medical and dental screening examination and subsequently were assigned to undergraduate students. The patients then received a complete dental and periodontal examination and appropriate treatment under the supervision of qualified instructors.

\subsubsection{Inclusion Criteria}

The study's target population $(\mathrm{n}=637)$ included all dentulous individuals (minimum of one tooth) aged $\geq 35$ years who had received a periodontal examination at the dental teaching clinic, Faculty of Dentistry, University of Manitoba during the period January 1991 - June 1994.

\subsubsection{Exclusion Criteria}

Individuals excluded from the study were those who, during the period January 1991 - June 1994: were under 35 years of age at the time of their periodontal examination, were edentulous or did not have a periodontal examination during the specified period.

Excluded from the target population were patients whose treatment needs were too complex or unsuitable for the undergraduate teaching program i.e., not accepted for care. These individuals included those whose treatment needs were beyond the level of training achieved by the students or those whose work or family commitments would make them unavailable for numerous, lengthy appointments. Patients who were accepted for 'limited treatment' and assigned exclusively to programs in undergraduate restorative dentistry or to the graduate programs in oral surgery or periodontics were not considered part of the target population. Those patients who were assigned exclusively to pedodontics, orthodontics or complete prosthodontics were also excluded from the target population. The 
rationale for excluding the aforementioned individuals included: inappropriate age ( $<35$ years), edentulous, or incomplete/inconsistent periodontal records.

Approximately 1,815 individuals received a screening examination during each full year and 907 in the remaining half year of the current study $[(1,815 \times 3)+(907)]=6,352$. Of these, approximately $15 \%(\mathrm{n}=953)$ each year were former FOD patients undergoing a re-screening. Out of everyone screened each year, it was estimated that $20 \%(n=1,270)$ were not accepted for treatment and another $2 \%(\mathrm{n}=127)$ were accepted for limited treatment (Cleghorn 1994). This would result in approximately 4,002 patients accepted for treatment over the study period $(6,352-2,350=4,002)$. However the 4,002 individuals included patients younger than 35 years $(\sim 54 \%)$ and others who do not meet the study's inclusion criteria including the edentulous $(\sim 4 \%)$, those referred to graduate programs in orthodontics, oral surgery and periodontics $(\sim 15 \%)$ and recall patients who did not have their initial periodontal examination during the study period $(\sim 11 \%)$. The latter group constituted the largest subpopulation. These individuals were recall patients i.e., patients who had attended the FOD for many years. Such individuals were excluded because their initial exam could have been from four to twenty years earlier when examination philosophy and clinical recording procedures differed from those of the study period. They were also excluded because their recall exposures may have differed from those examined during the past three and a half years. Due to the exclusion of large subgroups of individuals, it should be noted that the results of the study are not to be generalized beyond the target population $(n=637)$. The process of subject selection is outlined in Table 6.1. 


\subsection{SAMPLE SIZE AND STUDY POWER}

The simultaneous association of up to eight explanatory variables with the history of moderate to advanced periodontitis was investigated. The minimum required sample size was $\mathrm{n}=140$ cases and 140 controls. Number Cruncher Statistical System's Power Analysis and Sample Size Package (NCSS-PASS) was used to calculate minimum sample size (Hsieh 1989). There was adequate power (80\%) to detect an odds ratio (OR) of $\geq 2.0$ when the prevalence of any explanatory variable was $>12 \%$. Where the prevalence of an exposure was not at least $12 \%$, the exposure was included as part of a multi-risk variable, e.g. use of one or more study medications.

Sample size calculations considered: The prevalence of cigarette smoking in Canada in 1992 was 30\% (Stachenko et al 1992). An estimated $5 \%$ of Canadians have diabetes mellitus with $12 \%$ of those aged $55-74$ years affected (Reeder et al 1992). A recent study in Manitoba suggests the prevalence of diabetes to be $9 \%$ among 35 - 64 year olds and $15 \%$ among those $\geq 65$ years of age (Gelskey et al 1991). Among Canadian adults, the prevalence of hypertension (diastolic blood pressure $\geq 90 \mathrm{~mm} \mathrm{Hg}$ or undergoing treatment) is approximately $15 \%$ (Joffres et al 1992). The prevalence of hypertension among adult Manitobans has been shown to be $17 \%$ (Gelskey et al 1991). In the United States, $75 \%$ of those aged $\geq 65$ years report to take at least one prescription medication. Of these, the mean number of medications per individual was 14 (NCHSR 1982) Cardiovascular medications and hormones are among the top ten categories of prescribed medications (Blackmore 1994). It was anticipated that the prevalence of chronic diseases and conditions would be somewhat higher in the study population because it was assumed that they were from a low SES.

The response rate for the telephone interview was anticipated to be at least $70 \%$ due to the pre-study letter of endorsement sent to potential 


\section{TABLE 6.1 Subject selection process}

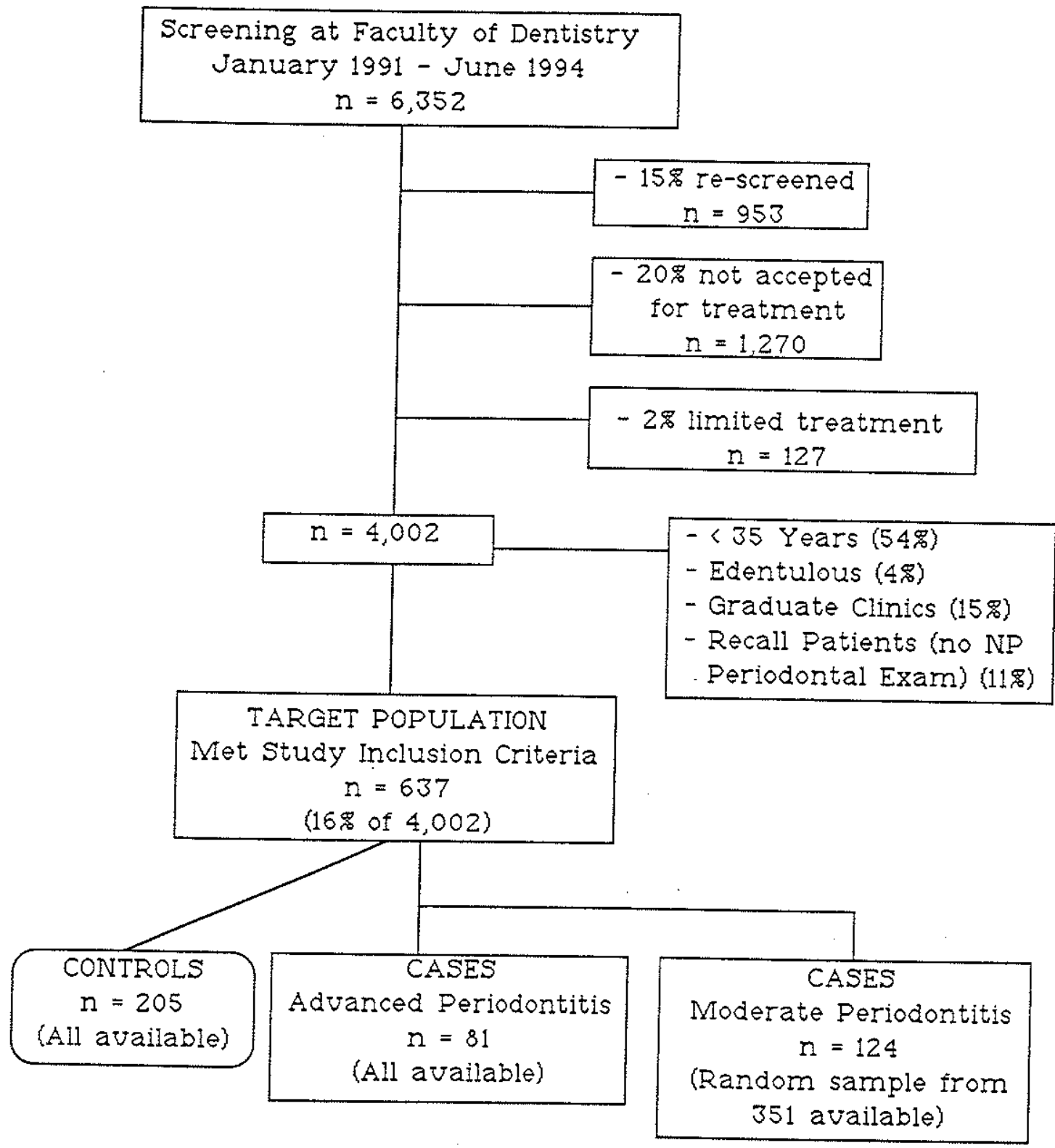


subjects by the Faculty of Dentistry (Appendix 6a). Based on this estimate of $30 \%$ non-response and the required minimum sample size (140/140) 204 cases and 205 controls were selected. One case was eliminated because the account had been sent to "collection".

\subsection{SAMPLING FRAME}

The sampling frame included all adult patients (aged $\geq 35$ years) who had been accepted for treatment in the undergraduate dental clinic and had received a periodontal examination and diagnosis during the study period.

\subsection{THE DEPENDENT VARIABLE:}

Information on the dependent variable was obtained from existing clinical and radiographic records by means of a chart review conducted by the Principle Investigator. The study's dependent variable was the binary status of the patients' periodontal history, i.e., case vs control.

\section{Selection of Controls:}

Using the Faculty's computerized patient data base and its designated periodontal classification system, control patients were retrieved by a computer specialist from Clinic Administration using an 'inhouse' patient retrieval program designed to identify patients who met the study's inclusion criteria.

Although the initial intention was to obtain only subjects from the most recent academic year (1993 - 94), it was necessary to broaden the timeframe to a three and one-half year period, January 1991 - June 1994 in order to obtain a sufficient number of control patients $(1994=48 ; 1993=56 ; 1992=$ $43 ; 1991=58)$. The limited number of control patients in the sampling frame is believed to be related to Faculty-imposed student requirements to treat 
more complex patients as well as to the study's age restriction $\geq 35$ years. The age restriction was made since the effects of periodontitis are not generally seen until after age 35 years (Suzuki 1988).

Adequate numbers of controls $(n=205)$ were obtained by including patients examined during the previous three and one-half years i.e., January 1991 - June 1994. All class 201, 202, 701 and 702 patients were selected as the control subjects. The distribution of controls by year of periodontal exam is presented in Table 6.2.

TABLE 6.2 Target population. Distribution of controls and cases by year of perio examination

\begin{tabular}{cccccc}
\hline & $\begin{array}{c}\text { Controls } \\
\text { 01's \& 02's }\end{array}$ & “04"s & $\begin{array}{c}\text { '03"'s } \\
\text { (total) }\end{array}$ & $\begin{array}{c}\text { Randomly } \\
\text { selected } \\
\text { 03's }\end{array}$ & $\begin{array}{c}\text { Cases } \\
\text { combined 04's and } \\
\text { randomly selected 03's }\end{array}$ \\
\hline 1991 & 58 & 18 & 86 & 35 & 53 \\
1992 & 43 & 22 & 81 & 28 & 50 \\
1993 & 56 & 24 & 93 & 30 & 54 \\
1994 & 48 & 17 & 91 & 30 & 47 \\
TOTAL & $\mathbf{2 0 5}$ & $\mathbf{8 1}$ & $\mathbf{3 5 1}$ & $\mathbf{1 2 3}$ & $\mathbf{2 0 4}$ \\
\hline
\end{tabular}

Distribution of Target Population of Controls and Cases

Controls: Classes 01, 02 = healthy, gingivitis or slight periodontitis. Case: Classes 03, $04=$ moderate - advanced periodontitis.

All available controls were recruited from each year. All available Class 04 cases were recruited from each year. A random selection of Class 03 cases $(n=123)$ were selected from the total available $(\mathrm{n}=351)$.

\section{Selection of Cases:}

Cases were retrieved from the same three and one-half year period as were controls. In order to maximize the chance of finding differences between cases and controls, all eighty one $(n=81)$ identified class 204, 704 (advanced periodontitis) patients were selected for the study. The remainder of the one hundred twenty three $(n=124)$ cases were selected 
randomly from the three hundred fifty one $(n=351)$ class $203 / 703$ patients in the data base using sequential selection with a random start.

\subsubsection{Case Definition}

For the purposes of the current study, "cases" were identified initially from the FOD computerized patient data base for the period under investigation (January 1991 - June 1994). The data base contained the periodontal classification (diagnosis) for each patient as determined at the clinical periodontal examination. The FOD's periodontal classifications 703 and 203 were indicative of moderate periodontitis and classes 704 and 204 were indicative of advanced periodontitis.

For the purposes of the current study, cases were subsequently defined using the following criteria:

Criterion A: $\quad \geq 2$ teeth with $\geq 5 \mathrm{~mm}$ probing depth (PD)

Criterion B: $\quad \geq 1$ teeth with $\geq 7 \mathrm{~mm}$ probing depth (PD)

Criterion C: $\quad \geq 1$ teeth with $>3 \mathrm{~mm}$ distance from cemento-enamel junction (CEJ) to the alveolar crest $\mathrm{AC}$ ) radiographically

Criterion D: $\quad$ the periodontal classification established at the initial periodontal exam and subsequently obtained from the computerized patient data base (classes 01, 02, 03, 04)

Criterion E: $\quad$ same as D except that classes 01 and 02 were combined into controls and classes 03 and 04 combined into cases

Criterion F: individuals who met Criterion B or C.

\subsubsection{Control Definition}

Periodontal classification i.e., classes 701 and 201 were indicative of gingivitis while classes 702 and 202 were indicative of slight periodontitis. Controls included individuals who were periodontally healthy as evidenced 
by absence of clinical inflammation and $\mathrm{PD} \leq 3 \mathrm{~mm}$ or by gingivitis and/or $\mathrm{PD} \leq 4 \mathrm{~mm}$ or by $\leq 3 \mathrm{~mm}$ distance from the $\mathrm{CEJ}$ to $\mathrm{AC}$ radiographically.

\subsection{INDEPENDENT VARIABLES: METHODS OF DATA COLLECTION}

\subsubsection{Chart Review}

Information on the following independent variables was obtained from a review of the dental/medical charts in the FOD:

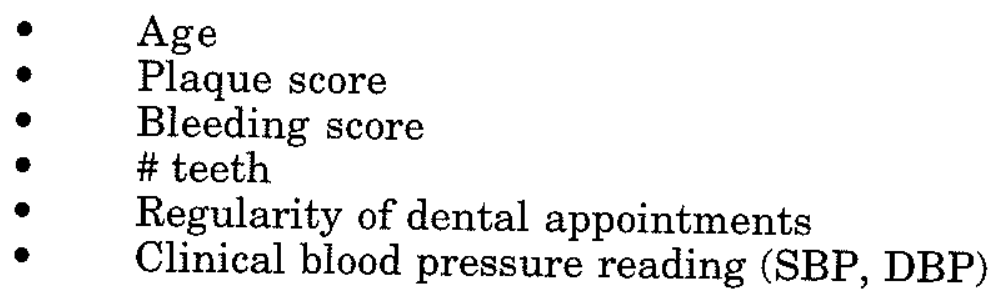

A review of dental records for each subject was conducted by the PI with the following information retrieved, photocopied, and entered into Lotus 1-2-3 spreadsheet and NCSS for subsequent analysis:

1. Chart number, date of birth, date of periodontal exam.

2. Original periodontal diagnosis as determined at the initial periodontal examination.

3. Date of corresponding bite-wing radiographs (BWX) or alternative type of radiograph where necessary. Radiographs were subsequently analyzed for evidence of bone loss.

4. Address and phone number(s).

5. Medical history forms which were obtained from the patient at the time of the initial periodontal examination (Appendix $6 \mathrm{~b}$ ).

6. Dental and periodontal history as recorded including: \# teeth present; \# teeth with $\geq 5 \mathrm{~mm} \mathrm{PD}$; \# teeth with $\geq 7 \mathrm{~mm} \mathrm{PD}$, status of Criteria $\mathrm{A} / \mathrm{B} / \mathrm{C} / \mathrm{D} / \mathrm{E} / \mathrm{F}$; plaque and bleeding score. Plaque and bleeding scores were calculated by the PI and included all teeth present. Prior to the study, it was determined that if the record was not available, that the students' calculated plaque and bleeding score would be used. Where neither was available, but a written description was used, a percentage would be assigned based on the following: 
- $25 \%=$ "slight/light/minimal plaque/gingivitis"

- $50 \%=$ "moderate/generalized plaque/gingivitis"

- $100 \%=$ "heavy/extensive plaque/gingivitis"

\subsubsection{Radiographic Analysis}

\subsubsection{Dentist Calibration}

Radiographic evidence of alveolar bone loss was assessed by an experienced independent dentist, blind to the clinical periodontal diagnosis of each subject. Prior to the study, the dentist participated in two calibration/standardization sessions with the Principal Investigator (PI). In the first session, the study protocol for BWX films was applied while the second session focused on the protocol for periapical (PA) and panoramic (PAN) radiographs. Bite-wing radiographs provided a view of the tooth crown's alveolar crest and interproximal area in posterior teeth. Periapical radiographs provided a view of an entire tooth and its periodontal supporting structures. Panoramic radiographs provided an overall, but less detailed view, of the entire dentition. Each calibration session involved assessment of a random selection of 20 patients' radiographs. The radiograph selected for calibration were randomly selected by the PI from radiographs processed at the FOD in 1990. All patients had previously attended the undergraduate clinic but were not potential participants in the current study. An equal number of patients had radiographic evidence of $>$ $3 \mathrm{~mm}$ bone loss on $\geq 1$ surface of a study tooth as those who did not.

Each radiograph was assessed independently by both the dentist and PI. The periodontal status (case/control) of only $1 / 40$ patients was classified differently by the two investigators. Differences in interpretation were discussed and the radiographic study protocols and forms clarified where necessary. 


\subsubsection{Study Conditions and Radiographic Equipment}

All radiographs were read by the same dentist using consistently controlled lighting conditions and equipment.

An Idealite view box, Richard Manufacturing Co., Van Nuys, California was used with no image magnification. A Mars Masterbow engineer's compass, model 551-OOWP by Staedtler Co., Germany was set at $3 \mathrm{~mm}$ using a Mitutoyo Venier Caliper reading of $3 \mathrm{~mm}$. The compass was locked into position by cold-solder. The compass setting was checked for calibration prior to each session of assessment. No change to the fixed reference distance was detected during the study.

Unmounted radiographs were placed in position on the viewbox. Measurements were made by placing one end of the compass on the CEJ i.e., the landmark where the outer edge of the enamel of the crown intersects the outer edge of the dentin of the root. The other end was placed adjacent to the root surface at the Alveolar Crest (AC) i.e., the most coronal point of bone adjacent to the tooth surface where the periodontal ligament space has a uniform width. If the distance between the CEJ and AC exceeded the span of the two compass points, the surface was labeled as having bone loss greater than $3 \mathrm{~mm}$. If two radiographs of the same tooth surface were available, the one with the more distinct landmarks was used. When in doubt, a surface was recorded as normal.

\subsubsection{Radiographic Assessment Protocols}

Radiographic assessment protocols for BWX and for PA/PAN films (Appendix 6c) were clearly documented and followed strictly. Assessment included determination of whether there was presence of $>3 \mathrm{~mm}$ distance between $\mathrm{CEJ}$ and $\mathrm{AC}$ on the mesial or distal of the second bicuspid or first or second molar in any quadrant. 
Individual surface evaluation: For each surface of the study teeth, one of the following codes was entered: " $x$ " when the tooth was missing or partially erupted; "?" when the surface was unreadable, whether due to overlapping image; cone-cut; CEJ or AC obscurement by caries/restoration/etc.; or improper film placement, exposure or processing; "+" when the two compass points were exceeded by the distance between the CEJ and AC; "--" when the distance between the CEJ and AC did not exceed the distance between the two compass points.

Overall Diagnosis: Based on the findings from the individual surface evaluation, "yes" was circled if a surface of any study teeth had evidence of a distance greater than $3 \mathrm{~mm}$ between the CEJ and AC i.e., a distance greater than the distance between the two compass points. "No" was circled if the distance between the CEJ and AC was equal to or less than the distance between the two points on the compass for all tooth surfaces under study. If an overall diagnosis could not be made due to unreadable films, "unreadable" was circled.

\subsubsection{Radiograph Types Assessed}

Where available, BWX radiographs were assessed. When the subjects' chart contained no BWX, periapical (PA) radiographs were used, sometimes in combination with a panoramic (PAN) radiographs. Whenever the case/control status was determined using a PA or PAN, it was indicated on the assessment form.

Completion of BWX assessment occurred for all subjects who had these films prior to the dentist's assessment of PA or PAN radiographs. 
This strategy was used to minimize confusion between radiograph-type assessment protocols.

All radiographs included in the study, although produced by various clinicians, were exposed and processed at the Faculty of Dentistry under the supervision of the same radiographic staff using consistent equipment and recommended exposure times for all techniques (Appendix 6d).

\subsubsection{Intra-Rater Reliability And Radiograph Type Reliability}

A random selection of all radiographs types (15 BWX and eight PA/PAN) was made during the study assessment sessions. Re-assessment of all types of radiograph was done for both cases and controls. The radiographs were re-assessed by the same dentist to determine intra-rater reliability. The dentist was blind to the fact that he had read the radiographs one week earlier. Kappa statistic was used to analyze the data. The results are reported in Chapter Eight.

To determine the reliability of PAs and PANs when compared to $B W X$ radiographs, 13 patient charts were randomly selected from patients whose BWX had already been read. The blinded dentist subsequently classified subjects based on their PA/PAN radiographs. Comparison was made between the radiograph-type assessments regarding ability to similarly categorize the case/control status. Results are reported in Chapter Eight.

\subsubsection{Choice OfTeeth, Surfaces, Radiograph Type And Distance}

Decisions to include the mesial and distal surfaces of the second premolar and first and second molars in all quadrants and to use the measurement of $>3 \mathrm{~mm}$ distance between the cemento-enamel junction and alveolar crest to represent a history of periodontitis and to examine 
BWX radiographs (when available) are based on the results of previous studies. The details of these studies are critiqued in Chapter Seven.

\subsubsection{Telephone Interview}

\subsubsection{Interview Design}

A structured telephone interview (Appendix 6e) was used to identify to which antecedent risk indicators cases and controls had been exposed. The independent variables assessed were:

I. Behavioral Variables including frequency of toothbrushing and flossing and history of cigarette smoking.

II. Chronic Diseases and Conditions including history of diabetes, hypertension, or ischemic heart disease (myocardial infarction, angina pectoris, and arterio/atherosclerosis)

III. Use of Specific Medications including dilantin/phenytoin, nifedipine/Adalat, cyclosporin, and estrogen/progesterone. Use of anti-inflammatory medications was also assessed.

IV. Socioeconomic Factors including income, education, occupation and marital status. The questions on occupation and income were taken from the Manitoba Heart Health Survey (Young et al 1991).

\subsubsection{Interviewer Training}

There were three interviewers involved in the study which included the Principal Investigator (PI) and two students in their second year of dental hygiene school. An Interviewer Training Manual (available on request from $\mathrm{PI}$ ) was developed by the PI to train and standardize all interviewers. The interviewers were blind to the case-control status of the subjects at the time of the interview. Beginning on November 8, 1994, three 
interviewer training periods of approximately one and one half hours each were conducted.

\subsubsection{Pre-Testing The Interview}

The questionnaire was pre-tested between November 16 - 18, 1994, among nine patients currently receiving care at the FOD but not selected for the study. An attempt was made to include both genders, a range of ages, individuals who demonstrated difficulty with the English language, and patients both with and without complex general and periodontal conditions.

Each interviewer acted as the primary interviewer of three individuals while the other two interviewers listened-in on the interview through a common line conference call. The respondent was informed prior to the interview that three individuals would be listening to his/her responses. All three interviewers scored the questionnaire responses independently. The PI subsequently reviewed the questionnaire forms. Each question was assessed to determine if it failed to be scored identically by all interviewers. For those questions which were scored differently, one of the following actions was taken. Either a minor change was made to the question in order to clarify the intent of the question or a 'prompt' was inserted into the questionnaire adjacent to the question. No major changes to the questionnaire were indicated.

\subsubsection{Interview Process}

Subjects ( $\mathrm{n}=409$ ) were assigned randomly to the three blind interviewers. Subjects had previously been listed numerically by patient chart number on 46 pages, each page containing approximately nine subjects. The date of the periodontal examination was written adjacent to each subjects' name. No information as to the case-control status was 
available to the interviewers. Unequal distribution of subjects to the three interviewers was anticipated due to undergraduate student time constraints. The PI anticipated carrying out 50 - 75 percent of the interviews.

Debriefing sessions were held with the interviewers after approximately a dozen interviews had been conducted by each interviewer and again at weekly intervals for three weeks during the interview process. Following each debriefing session, the principal investigator went through the completed questionnaires to ensure that all data entry was complete and accurate. Any discrepancies were discussed with the interviewers. Cross-references were made by the PI between the questionnaire and the subjects' photocopied chart when necessary to reduce interviewer error. The interviews were conducted between November 19, 1994 and January 5, 1995, approximately seven weeks.

Each interview was rated by the interviewer on its quality. Criteria included: Respondent's understanding of questions; Respondent's recall of information; and The chance of getting the same response in two weeks. A Lickert scale was used to rate the interview's quality from $1=$ low to $5=$ high. It was determined a priori that any completed questionnaire that was rated "low" (i.e., 1) or "below average" (i.e., 2) question \#1 or \#2 would be eliminated from the study due to unreliability of the interview.

\subsubsection{Inter-Rater Reliability And Test-Re-Test Reliability}

Within two weeks of the first interview, a number of subjects were reinterviewed to determine the inter and intra-rater reliability. Since the PI completed the majority of the interviews, she re-interviewed 20 of her own interviews. In addition, she re-interviewed five each of the other two interviewers' subjects. The other two interviewers each re-interviewed five 
of the PI's interviews as well. Subjects who participated in the reinterviewing process had been randomly selected by the PI from lists of previously completed interviews. An inter/intra-rater reliability of $80 \%$ was set a priori (Bergner and Rothman 1987). Results are reported in Chapter Eight.

\subsection{CONCORDANCE OF DIAGNOSTIC CRITERIA}

The most valid measure of a history of adult periodontitis is evidence of periodontal attachment loss. The two most accepted methods of determining attachment loss are clinical probing attachment loss and radiographic evidence of alveolar bone loss. The later method was used as evidence of periodontal attachment loss in the current study but not the former because the former measurements were unavailable. Radiographic evidence of $>3 \mathrm{~mm}$ distance between the alveolar crest and cemento-enamel

junction was established as the gold standard and indicative of a history of periodontitis, i.e., "case". The validity of periodontal measurements and diagnostic criteria are discussed in Chapter Seven.

Agreement of other clinical criteria with the radiographic gold standard was analyzed in an attempt to identify the optimum diagnostic criterion or combination of criteria to employ in multivariate analyses. Contingency tables were used to calculate the sensitivity of the diagnostic criterion, that is, the proportion of those with disease who were correctly classified by the test as having the disease; and specificity, that is, the proportion of those who truly do not have the disease that were correctly classified by the test as not having it. Measurement of a binary outcome (disease/not disease) is perfect only when both sensitivity and specificity are 100\% (Kelsey et al 1986). Kappa statistic and $\mathrm{Z}$ values were also calculated 
for each pair of diagnostic criteria. Diagnostic Criteria were defined in Section 6.4.1.

Criterion B was combined with Criterion $\mathrm{C}$ to form new Criterion $\mathrm{F}$ which included individuals who had radiographic evidence of $>3 \mathrm{~mm}$ bone loss 'or' those who lacked radiographic evidence but had $\geq 1$ site with $\geq 7$ $\mathrm{mm}$ PD. This was done to maximize the number of cases. It was this combined criterion (F), which demonstrated the greatest sensitivity (100\%) and specificity (89\%) Kappa 0.89, SE $=0.05, \mathrm{Z}=17.9, \mathrm{p}<0.0001$. Results are reported in Table 6.3.

TABLE 6.3 Concordance of criteria

I. Radiographs ("gold standard") Criterion C (n = 359)

\begin{tabular}{|c|c|c|c|c|}
\hline & SENSITIVITY & SPECIFICITY & KAPPA & PVALUE \\
\hline $\begin{array}{l}\text { 01/02/03/04 } \\
\text { (Criterion D) }\end{array}$ & $37.6+31.2=68.8$ & $16.8+52.0=68.8$ & - & - \\
\hline $\begin{array}{l}\text { Case/control } \\
\text { (Criterion E) }\end{array}$ & 68.8 & 68.8 & 0.375 & $\mathrm{p}<0.02$ \\
\hline $\begin{array}{l}\geq 2 \text { sites } \geq 5 \mathrm{~mm} \\
\text { (Criterion } A)\end{array}$ & 67.7 & 68.8 & 0.363 & $\mathrm{p}<0.001$ \\
\hline $\begin{array}{l}\geq 1 \text { site } \geq 7 \mathrm{~mm} \\
\text { (Criterion } \mathrm{B} \text { ) }\end{array}$ & 36.6 & 89.0 & 0.251 & $\mathrm{p}<0.02$ \\
\hline $\begin{array}{l}\text { Combo Rad or } 7 \\
\text { mm (Criterion F) }\end{array}$ & 100.0 & 89.0 & 0.893 & $p<0.0001$ \\
\hline
\end{tabular}

$7 \mathrm{~mm}$ alone was not sensitive identifying approximately $1 / 3$ of cases. Two sites of $\geq 5 \mathrm{~mm}$ PD identified about $70 \%$ of cases. Radiographs or $7 \mathrm{~mm}$ identified $100 \%$ of cases and the specificity indicated that only $11 \%$ of undiseased people were labelled as diseased.

II. Combination of radiographic evidence or $\geq 7 \mathrm{~mm}$ PD ("gold standard") Criterion F ( $\mathrm{n}=362)$

\section{1/02/03/04}

Criterion $\mathrm{D}$

Case/control

Criterion E

$5 \mathrm{~mm}$ Criterion A

$7 \mathrm{~mm}$ Criterion B
71.2

69.2

43.3
76.0

74.7

100.0 $\mathrm{p}<0.001$

$0.39 \quad \mathrm{p}<0.001$ 


\subsubsection{Differences Between Cases and Controls: Based on Six Diagnostic Criteria}

The Chi-square test (cross tabs) was carried out for each diagnostic criterion against all independent variables. An ANOVA and t-test were used for the continuous variables.

The results indicated that there were variations in differences observed between cases and control when the six criteria were examined. Results are reported in Table 6.4. These results suggest that not all criteria were equivalent/redundant in their relationship to explanatory variables. Criteria did not appear to be proxies for one another. The findings suggest that Criterion $\mathrm{A}$ is actually more closely related to gingivitis than periodontitis since it provides no evidence of bone/attachment loss (Goodson 1990; Theil and Heany 1991). Criteria D and E are based on the existing clinical diagnosis at the periodontal examination and may in fact fail to consider previous bone loss. Categorization of subjects based on these latter criteria may also include patients for whom measurement of $\geq 5 \mathrm{~mm}$ probing depth alone denoted periodontitis, while as stated previously, such depth may be due to gingivitis and not periodontitis. The former situation would result in an underestimation of cases, the latter, an overestimation. Either would bias the results of the study by over/under estimating the risk associated with particular variables (Table 6.4).

\subsection{Ethical and Access Considerations}

Ethical approval for the study was obtained from the Committee on Research Involving Human Subjects, Faculty of Dentistry in February 1994 (Appendix 6f).

As to individual patient consent, a pre-interview letter of information was sent on behalf of the PI by the Dean, FOD, to all potential subjects ( $\mathrm{n}=$ 
TABLE 6.4 Significant difference found between case and control according to explanatory variables
by diagnostic criteria

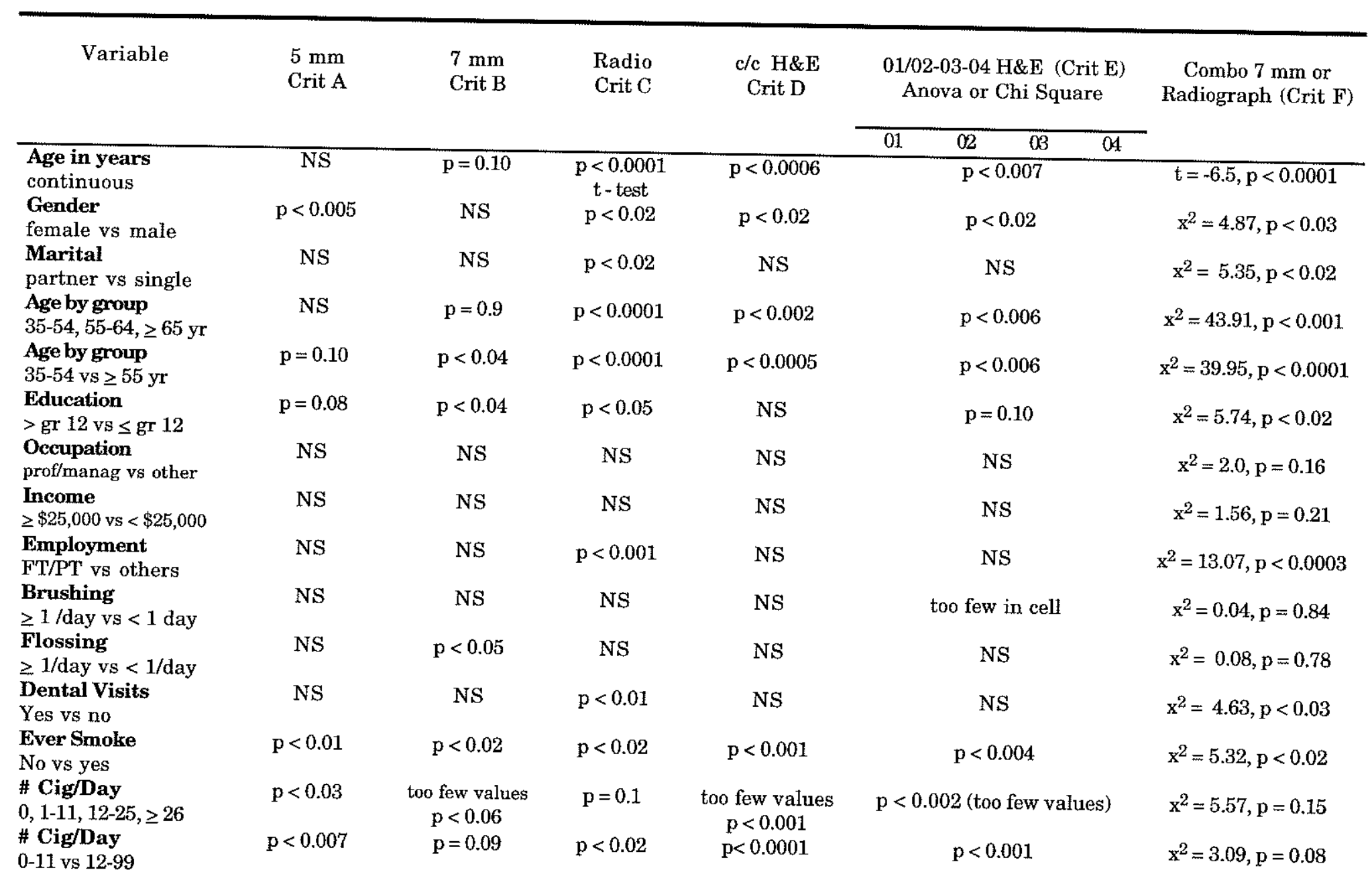




\begin{tabular}{|c|c|c|c|c|c|c|}
\hline $\begin{array}{l}\text { Cig index } \\
0,1-250,251-500,>500\end{array}$ & $\begin{array}{l}x^{2}=17.76 \\
p<0.0005\end{array}$ & $\begin{array}{c}x^{2}=14.60 \\
p<0.002\end{array}$ & $\begin{array}{l}x^{2}=11.58 \\
p<0.009\end{array}$ & $\begin{array}{l}x^{2}=27.65 \\
p<0.0001\end{array}$ & $p<0.0003$ (few cell values) & $x^{2}=16.43, p<0.009$ \\
\hline $\begin{array}{l}\text { Diabetes } \\
\text { no vs yes/borderline }\end{array}$ & $\mathrm{p}<0.03$ & $\mathrm{p}=0.1$ & $\mathrm{p}<0.05$ & $\mathrm{p}<0.03$ & $\mathrm{p}=0.10$ (too few values) & $\mathrm{x}^{2}=2.67, \mathrm{p}=0.10$ \\
\hline $\begin{array}{l}\text { Diag Hypertens } \\
\text { no vs yes }\end{array}$ & $p=0.10$ & NS & $\mathrm{p}<0.001$ & $\mathrm{p}<0.005$ & $p<0.02$ & $\mathrm{x}^{2}=6.40, \mathrm{p}<0.01$ \\
\hline $\begin{array}{l}S B P>140 \\
\text { no vs yes }\end{array}$ & $p=0.10$ & $p=0.09$ & NS & $\mathrm{p}<0.01$ & $\mathrm{p}=0.08$ & $x^{2}=3.62, p=0.05$ \\
\hline $\begin{array}{l}\text { DBP }>\mathbf{9 0} \\
\text { no vs yes }\end{array}$ & NS & NS & NS & NS & NS & $\mathrm{x}^{2}=1.33, \mathrm{p}=0.24$ \\
\hline HBP Combo & $\begin{array}{c}\mathrm{x}^{2}=3.92 \\
\mathrm{p}<0.05\end{array}$ & $p=0.19$ & $\begin{array}{l}x^{2}=7.12 \\
p<0.008\end{array}$ & $\begin{array}{l}x^{2}=8.05 \\
p<0.005\end{array}$ & $\mathrm{p}<0.03$ & $\mathrm{x}^{2}=6.69, \mathrm{p}<0.009$ \\
\hline $\begin{array}{l}\text { Any ischemic } \\
\text { no vs yes }\end{array}$ & NS & NS & $\mathrm{p}<0.04$ & $p=0.10$ & $p<0.05$ (too few in cell) & $x^{2}=3.97, p<0.05$ \\
\hline $\begin{array}{l}\text { Anti-inflammatory } \\
\text { no vs yes }\end{array}$ & NS & NS & NS & NS & NS & $\mathrm{x}^{2}=3.05, p=0.08$ \\
\hline $\begin{array}{l}\text { Estrogen } \\
\text { no vs yes }\end{array}$ & NS & NS & NS & NS & NS & $\mathrm{x}^{2}=2.63, \mathrm{p}=0.11$ \\
\hline $\begin{array}{l}\text { Stress } \\
\text { No/slight vs mod/severe }\end{array}$ & NS & NS & NS & NS & NS & $\mathrm{x}^{2}=1.17, \mathrm{p}=0.28$ \\
\hline $\begin{array}{l}\text { Plaque } \\
0-33,34-66, \geq 67 \%\end{array}$ & $\mathrm{p}<0.002$ & $p<0.009$ & $\mathrm{p}<0.04$ & $p<0.02$ & $\mathrm{p}<0.001$ & $\mathrm{x}^{2}=9.30, \mathrm{p}<0.009$ \\
\hline $\begin{array}{l}\text { Plaque } 67 \% \\
<67 \% \text { vs } \geq 67 \%\end{array}$ & $\mathrm{p}<0.001$ & $\mathrm{p}<0.002$ & $\mathrm{p}<0.02$ & $p<0.01$ & $\mathrm{p}<0.001$ (too few in cell) & $\mathrm{x}^{2}=14.45, \mathrm{p}<0.0001$ \\
\hline $\begin{array}{l}\text { Plaque } 50 \% \\
<50 \% \text { vs } \geq 50 \% \\
\text { POP }\end{array}$ & $\begin{array}{l}x^{2}=15.70 \\
p<0.0001\end{array}$ & $\begin{array}{l}\mathrm{x}^{2}=4.33 \\
\mathrm{p}<0.04\end{array}$ & $\begin{array}{l}x^{2}=7.28 \\
p<0.007\end{array}$ & $\begin{array}{l}x^{2}=5.57 \\
p<0.02\end{array}$ & $\mathrm{x}^{2}=25.67$ & $\mathrm{x}^{2}=7.50, \mathrm{p}<0.006$ \\
\hline $\begin{array}{l}\text { BOP } \\
0-33,34-66, \geq 67 \%\end{array}$ & $\mathrm{p}<0.001$ & $\mathrm{p}<0.001$ & $\mathrm{p}<0.001$ & $\mathrm{p}<.0001$ & $\mathrm{p}<0.0001$ & $x^{2}=21.97, p<0.0001$ \\
\hline $\begin{array}{l}\text { BOP } 67 \% \\
<67 \% \text { vs } \geq 67 \%\end{array}$ & $p<0.007$ & $\mathrm{p}<0.0001$ & $p=0.07$ & $\mathrm{p}<0.0009$ & too few values & $\mathrm{x}^{2}=10.57, \mathrm{p}<0.001$ \\
\hline $\begin{array}{l}\text { BOP 50\% } \\
<50 \% \text { vs } \geq 50 \% \\
\text { \# teeth }\end{array}$ & $\begin{array}{l}\mathrm{x}^{2}=20.73 \\
\mathrm{p}<0.0001\end{array}$ & $\begin{array}{c}x^{2}=18.11 \\
p<0.0001\end{array}$ & $\begin{array}{l}x^{2}=7.12 \\
p<0.008\end{array}$ & $\begin{array}{c}x^{2}=11.95 \\
p<0.005\end{array}$ & $\mathrm{p}<0.0001$ & $\mathrm{x}^{2}=10.91, \mathrm{p}<0.001$ \\
\hline$<10$ present $\geq 10$ present & $\mathrm{p}<0.02$ & & NS & $\mathrm{p}<0.05$ & $\mathrm{p}<0.05$ (too few values) & $\mathrm{x}^{2}=0.07, \mathrm{p}=0.79$ \\
\hline $\begin{array}{l}\# \text { teeth } \\
<20, \geq 20\end{array}$ & NS & NS & NS & NS & NS & $\mathrm{x}^{2}=1.26, \mathrm{p}=0.26$ \\
\hline $\begin{array}{l}\# \text { teeth } \\
<25, \geq 25 \\
\end{array}$ & NS & NS & $\mathrm{p}<0.0001$ & $p<0.002$ & $\mathrm{p}<0.01$ & $\mathrm{x}^{2}=27.64, \mathrm{p}<0.0001$ \\
\hline
\end{tabular}


409) (Appendix 6a). The letter described the study briefly, and that participation, although encouraged, was voluntary. Upon initial telephone contact, a telephone script (Appendix 6g) was read by the interviewer to each potential participant. The script reiterated the study goals and obtained informed consent. When English was not understood, arrangements were made within the respondent's family for interpretation of consent issues as well as for the interview. Only those who consented to participate $(n=382,93.4 \%)$ were interviewed.

All participants wishing a summary of the study's findings were told that they would receive a report after the completion of the thesis.

Access to the Faculty of Dentistry's Patient Data Base was made through consultation with the Dean of the Faculty of Dentistry and the Faculty's Head of Clinics and Patient Care. Retrieval of information from the Patient Data Base and Clinical Records was facilitated by staff from the Computer Department and the General Clinic.

\subsection{STUDY LIMITATIONS}

The case-control design of the current study presented limitations due to its inability to establish temporality. Study results therefore suggest associations rather than causes. However an attempt was made to manage temporal requirements by formulating time-specific interview questions, "Prior to 19_, did you ever...". Thus attempting to establish exposure to variables antecedent to the subjects' date of periodontal examination and diagnosis.

The response rate for the interview was adequate (93.4\%) enhancing external validity and generalizability of results to the target population. However, results may not be generalized beyond the target population to the general population. In fact, caution should be taken not to apply the results 
to everyone seeking care at the FOD. The reason being that $20 \%$ of those undergoing screening exams are not accepted for treatment at the Faculty and others were excluded from the study due to reasons such as edentulism, being less than 35 years of age or being a FOD recall patient. These individuals are different from the target population in a number of ways, e.g. age, methods of diagnosis, extent of dental intervention, etc..

Use of secondary clinical data (plaque score, bleeding on probing, probing depth, \# teeth present) had limitations. The data were collected by many different students over the study period. Although reliability of these data is unknown, it was likely improved by consistency of clinical forms and evaluation/confirmation of diagnosis by teaching staff. Unstandardized radiographs are not as reliable as radiographs which are taken under standardized conditions which ensure reproducibility of film placement, angulation and exposure. However the latter were unavailable and may not be practical in large epidemiologic studies. The advantages of incorporating existing radiographic evidence of periodontal attachment loss (bone loss) in the current study outweighs the disadvantage of using unstandardized radiograph and use of three types of radiographs. The retrospective nature of the study did not permit use of clinical attachment loss as the data was unavailable. However, use of radiographic evidence plus conservative use of $\mathrm{PD}$ ( $\geq 7 \mathrm{~mm}$ PD) was believed to be an adequate proxy for CAL.

Lack of well-constructed questions on the pre-existing medical records precluded validation of many medical questions in the telephone interview questionnaire. It was possible however to validate several key variables, age, gender and history of diabetes and hypertension. No attempt was made to further validate medical findings or use of medications through consultations with subjects' physicians. 
Use of diagnostic criterion $F$ (i.e. $\geq 1$ surface with $\geq 7 \mathrm{~mm} P D$ or radiographic evidence $>3 \mathrm{~mm}$ distance between $\mathrm{AC}$ and $\mathrm{CEJ}$ ) to define cases could have systematically underestimated the number of cases. It is possible that some individuals with AP were not included as cases despite the fact that they may have had two mm clinical attachment loss. Using a cut-off of $>3 \mathrm{~mm}$ is consistent with other studies and attempts to account for measurement error. The associated limitation however is that there could be an underestimate of the risk associated with explanatory variables, i.e., an acceptance of the null hypothesis, type II error.

The effect of social desirability bias for the interview questions, "frequency" of toothbrushing/"flossing" and "history of smoking" is unknown. Over-reporting could have underestimated the risk associated with infrequent preventive measures. Recall bias could have influenced reported exposure to less salient variables. Key variables investigated were likely salient to subjects. It is doubtful that there was a systematic recall bias between cases and controls, since neither would be expected to over/under report these behaviors more than the other.

Another limitation of this study is that the risk indicators and markers identified provide an historical record of periodontal breakdown. The results may have no bearing on subjects' current or future periodontal health. Historical record of destruction was acceptable since the purpose of the study was to determine which variables were associated with a history of periodontitis, not active or future disease. 


\section{CHAPTER 7}

\section{VALIDITY AND RELIABILITY OF MEASUREMENTS}

\subsection{VALIDITY AND RELIABILITY}

\subsubsection{Types of Validity}

Validity is the extent to which an instrument measures what it purports to measure (Kelsey et al 1986). Several types of validity were considered in the current study:

1) FACE or LOGICAL VALIDITY is the extent to which a measurement logically/obviously appears to be relevant. For example, the date of birth recorded on a birth certificate provides a valid measure of age (Abramson 1990). In the present study, radiographic evidence of bone loss has face validity as a measure of a history of periodontal attachment loss.

2) CRITERION or CONCURRENT VALIDITY is the extent to which measurements of the phenomenon under study correlate with an external criterion. For example, a visual inspection of a wound for evidence of infection could be validated against the external criterion, bacteriological examination (Last 1988). In the current study, concurrent criterion validity was assessed through an analysis of the correlation between the study's Diagnostic Clinical Criteria and the 'gold standard', radiographic bone loss.

3) CONTENT VALIDITY is the extent to which measurement of a composite variable includes assessment of all of the relevant component elements of that variable. For example, a measurement of functional health status should include activities of daily living, occupational, family, and social functioning, etc. (Last 1988). In the current study, 'dental health 
compromising behavior' was assessed by measuring toothbrushing and flossing frequency as well as frequency of dental visiting and cigarette smoking.

Content validity for the phenomenon, 'history of periodontitis', was determined by a thorough review of the literature and in consultation with "experts" (periodontists and an oral pathologist) in the field. These sources indicate that measurements relevant to a history of periodontitis include plaque score (PS), bleeding on probing (BOP), probing depth (PD), and clinical attachment loss or radiographic evidence of alveolar bone loss (Machtei et al 1992). The current study assesses the relationship among these measures and their association with a history of periodontitis.

4) CONSTRUCT or CONVERGENT VALIDITY is the extent to which a measurement corresponds to theoretical concepts (constructs) concerning the phenomenon under study. For example, if on theoretical grounds, the phenomenon should change with age, a measurement with construct validity would reflect such a change (Last 1988; Abramson 1990).

The designated Diagnostic Criteria and their expected correlates were included in the study based on accepted theoretical concepts of their relationship to a history of periodontitis. The construct of a history of periodontitis has been represented or quantified by measurements of periodontal attachment loss as well as by probing depth (Clerehugh and Lennon 1984; Griffiths et al 1988; Machtei et al 1992).

Factors known to influence or be correlated with periodontitis such as plaque, bleeding on probing, smoking, age, sex and income (Papapanou et al 1988; Grbic et al 1991; Haffajee et al 1993) were also measured in the current study. There are theoretical reasons for believing that plaque is 
correlated with income and evidence of such a correlation in the current study could be taken as indirect evidence of construct validity. An additional test of construct validity involves plaque level assessment to validate questions on toothcleaning practices.

Results of tests of Validity are reported in Chapter Eight.

\subsubsection{Reliability}

Reliability is the extent to which an instrument produces the same result when repeatedly applied to the same person under the same circumstances (Kelsey et al 1986). Reliability refers to the degree to which the results obtained by a measurement procedure can be replicated by the same or another examiner (Last 1988). In the present study, intra-rater or test-re-test reliability was evaluated for the radiographic assessment of bone loss, as well as for telephone interviews using Kappa statistic or Intraclass Correlation Coefficients.

\subsection{SECONDARY CLINICAL DOCUMENTS}

Among the signs of a history of periodontitis are clinical attachment loss/level (CAL), radiographic evidence of bone loss, probing depth (PD), bleeding on probing (BOP), and plaque. Some of these parameters are related to a history of periodontitis by hypothesis primarily and have been shown to be variable in their association with periodontal diseases (Haffajee et al 1993). Only two, attachment loss and bone loss, are consistently associated with periodontitis. These two parameters describe and give objective evidence of the destructive nature of periodontitis without relying on a mechanistic hypothesis (Haffajee and Socransky 1986; Goodson 1990). Abramson (1990) states that a good operational definition is one which is phrased in observable facts. The observations of attachment loss and bone 
loss are hence automatically, 'by definition', valid as measures of periodontitis.

\subsubsection{Clinical Attachment Loss/Level}

\section{Validity:}

Clinical attachment loss is a measure, over time, of the change in position of the clinical attachment level (Griffiths et al 1988). Clinical attachment loss is an objective indication of decreased tooth support. Clinical attachment level is the distance, as measured by a periodontal probe, between the apical depth of the probe tip penetration and the cemento-enamel junction (CEJ) or another permanent reference point (Clerehugh and Lennon 1984; Griffiths et al 1988).

The criterion validity of connective tissue attachment level as a measure of early periodontitis has been assessed previously among 57 subjects requiring tooth extraction. Subsequent to clinical measurement of clinical attachment level, teeth were extracted, stained, and the histologic attachment level assessed. There were no significant differences between the mean clinical and laboratory measures of attachment on the buccal and mesio-buccal surfaces indicating that $\mathrm{CAL}$ is a valid measure of histologic connective tissue attachment loss (Clerehugh and Lennon 1984). Although there were no differences between mean measurements, there may have been differences between individual sites had they been analyzed. These results are consistent with other histological studies which have shown the difference between clinical and laboratory estimates of attachment loss averaged less than $0.4 \mathrm{~mm}$ (Clerehugh and Lennon 1984).

Clinical attachment level appears to be directly related (criterion validity) to periodontal destruction although some argue that indisputable proof may only be obtained histologically, post factum. i.e., clinical 
measurements only 'estimate' histological attachment (Machtei et al 1992) due to inherent measurement errors. Related errors are discussed in Section 7.2.3.)

A history of periodontitis may be identified through a single clinical attachment level measurement. However, a single measurement provides no information regarding the current periodontal disease status and may not be indicative of future disease. Repeated measurements indicating a change in CAL over time would be required for these purposes (Goodson 1990). For the purposes of the current study CAL refers to the single measurement of clinical attachment 'level' not to clinical attachment 'loss'.

The validity of ascribing buccal attachment loss to a history of chronic periodontitis has been questioned. It has been suggested that in studies of early periodontitis, measurement of CAL be restricted to proximal surfaces since buccal loss of attachment $\geq 1 \mathrm{~mm}$ has been attributed not only to periodontitis, but also to vigorous toothbrushing habits and associated apparent gingival recession (Clerehugh et al 1988). In studies involving moderate-advanced periodontitis however, buccal measurements are more meaningful (Clerehuh and Lennon 1984).

\section{Reliability:}

Probing CAL measurements are affected by many of the same errors as are PD measurements (discussed in Section 7.2.3) in detecting the most apical extent of the pocket. However, CAL measurements are more reliable indicators of a history of periodontitis than PD because they incorporate the permanent landmark of the CEJ (Griffiths et al 1988). Unfortunately, measurement error may sometimes be introduced due to the difficulty in accurately locating the CEJ (Badersten et al 1984). 
Measurement of CAL is associated with a relatively high error rate when measurement is made with conventional probes calibrated at $1 \mathrm{~mm}$ intervals, $\mathrm{SD}=0.82-1.16 \mathrm{~mm}$ (Haffajee et al 1983; Badersten et al 1984); and when a stent is used, $\mathrm{SD}=0.67-0.78$ (Aeppli et al 1984; Badersten et al 1984). When standardized probing forces alone are used, there is little effect on variability but when higher resolution electronic instruments are used with a fixed reference point, the standard deviations of differences are reduced to $0.2-0.3 \mathrm{~mm}$ (Jeffcoat et al 1986; Gibbs et al 1988).

Systematic measurement errors of CAL have been attributed to the influence of other concurrent clinical findings. CAL was often overestimated in the presence of gingivitis and underestimated in its absence (Clerehugh and Lennon 1984). These findings could be related to examiner bias or to probe penetration into connective tissue in the presence of inflammation.

\section{Summary:}

Ideally, the current study would have incorporated CAL as one of its diagnostic criteria. However, due to the study's retrospective nature, and the unavailability of these measurements, existing data from clinical records, $\mathrm{PD}$ and radiographs were used to determine subjects' history of periodontitis.

\subsubsection{Radiographic Evidence of Bone Loss}

\section{Validity:}

Alveolar bone loss is also considered a valid measure of a history of periodontitis because it is directly related to periodontal destruction, is hypothesis-free and objectively measured (Goodson 1990). 
While only repeated standardized radiographs are able to determine periodontal disease activity or progression, conventional radiographs provide a history of alveolar bone destruction (Hirschmann 1987). Radiographs have been used in both clinical and epidemiological studies to measure the prevalence of periodontitis. (Bjorn and Holmberg 1966; Blankenstein et al 1978; Jenkins and Mason 1984; Kallestal and Matsson 1991).

\subsubsection{Radiographic Criteria for Diagnosis of Periodontitis}

A number of different radiographic criteria have been suggested for the diagnosis of periodontitis including: marginal widening of the periodontal ligament; alveolar crestal irregularity; or evidence of a distance $\geq 2 \mathrm{~mm} />3 \mathrm{~mm}$ between the CEJ and AC. It is only the latter criterion which has gained general acceptance as having criterion validity (Hull et al 1975; Blankenstein et al 1978; Hirschmann 1987; Hausmann et al 1991; Caton 1993).

The other criteria are not accepted because: 1 ) the critical width of the periodontal ligament space indicative of periodontitis has not been determined. It is known that the ligament varies on radiographs depending on the radius of the circumference of the root as well as on the degree of occlusal trauma (Blankenstein et al 1978). 2) Irregularity of alveolar crest is not widely accepted as it has not been shown consistently to be a valid indicator of attachment loss (Blankenstein et al 1978).

While a history of moderate to advanced periodontitis is generally apparent radiographically, the diagnosis of slight/early periodontitis is more challenging (Hausmann et al 1991; Caton 1993). Some investigators suggest that a distance of $>2 \mathrm{~mm}$ between the CEJ and AC warrants a diagnosis of periodontitis (Hansen et al 1984; Gjermo et al 1984; Kaimenyi 
and Ashley 1988; Papapanou et al 1991; Kallestal and Matsson 1991) while others propose that the diagnosis is only warranted when there is evidence of a distance of $>3 \mathrm{~mm}$ (Hull et al 1975; Blankenstein et al 1978; and Davies et al 1978; Hausmann et al 1991). These differences may be related to inconclusive evidence regarding the optimum distance of the alveolar crest in relation to the CEJ in health (Schei et al 1959; Stoner 1972).

A previous clinical study investigated the radiographic measurement between the CEJ and AC among individuals in whom there was no clinical loss of attachment. A range of 0.3 to $2.6 \mathrm{~mm}$ distance between the $\mathrm{CEJ}$ and $\mathrm{AC}$ was detected using $\mathrm{BWX}$ radiographs in the absence of any clinical attachment loss. The results suggest that a conservative distance of $>3 \mathrm{~mm}$ between the CEJ and $\mathrm{AC}$ is the most valid indicator of incipient/early periodontitis (Hausmann et al 1991).

Radiographs inherently result in a conservative estimate of the periodontal history since radiographs underestimate slight periodontitis (Akesson et al 1992). Underestimation occurs because: 1) radiographically, all deviations from optimal beam angulations reduce the measurable distance from the $\mathrm{CEJ}$ to $\mathrm{AC} ; 2$ ) affected teeth/surfaces may be excluded due to difficulties in identifying either the CEJ or AC; 3) anterior teeth are often excluded from radiographic studies; 4) a radiograph is a two-dimensional image of a three-dimensional object, therefore the buccal and lingual bone levels can not be easily assessed on BWX; 5) BWX are unable to detect early bone loss occurring in the medullary defect located in the crater-like area between the buccal and lingual cortical plates, the site where bone destruction first and most frequently manifests itself; 6) anatomical variation among teeth may result in measurement error; 7) changes in attachment level precedes radiographically evident crestal bone changes by 
about six months (Bender and Seltzer 1961; Stoner 1972; Becker et al 1979; Goodson et al 1984; Hirschmann 1987; Hausmann et al 1991).

Despite the limitations of radiographs to determine periodontal history, a study of the criterion validity of radiographs has shown that bone loss on dry skulls when measured directly and radiographically result in measurements that were the same or differed by only $+/-1 \mathrm{~mm}$ in $90 \%$ of the measurements (Stoner 1972).

\subsubsection{Validity of Partial-Mouth Radiographs}

Although full-mouth series of periapical radiographs (PAs) have been used to assess the prevalence of periodontitis, partial-mouth measurements are more common, especially for epidemiological studies (Davies et al 1978; Papapanou et al 1991; Kallestal and Matsson 1991). The choice of teeth in a partial-mouth assessment influences the internal and external validity of the measurements. A study of the criterion validity and reliability of a partial-mouth assessment found that bone loss measurements from mandibular posterior areas were representative of full-mouth bone-loss measurements. Mandibular second premolars plus any other mandibular posterior teeth were the optimal combination for an abbreviated index (Shrout et al 1990). Since these teeth are included in standard bite-wing radiographs (BWX), it may be concluded that BWX radiographs are representative of the individual's periodontal condition and are appropriate for epidemiological studies.

Further support for the use of molars and premolars for epidemiological studies comes from studies of adolescents, among whom there was little evidence of periodontitis $(0.06-11 \%)$, and when detected, affected the maxillary first molars most frequently. Others have also found that the majority of early lesions were confined to first molars (Hansen et al 
1984; Gjermo et al 1984; Kallestal and Matsson 1991). In addition, the mandibular/molar region is the most reliable area of the mouth for collection of radiographic bone loss measurements since these sites are not subject to as much superimposition of anatomic structures and angulation errors as the anterior area (Shrout et al 1990).

\section{Reliability:}

Although radiographic measurement of bone loss suffers from low sensitivity and high error levels (Goodson 1990), it is used to increase the reliability of the periodontal examination (Ryan 1985; Polson and Goodson 1985; Griffiths et al 1988; Shrout et al 1990).

\subsubsection{Relative Reliability of Radiographic Techniques}

Three radiographic techniques are used to diagnose periodontitis: The bite-wing (BWX), the periapical (PA) and the panoramic x-ray (PAN).

\section{BWX Radiographs:}

The BWX technique aligns the long axis of the tooth parallel to the film and directs the x-ray beam at a right angle to this plane. This BWX alignment produces an accurate representation of the alveolar crest on the radiograph with only slight magnification (Ramadan and Mitchell 1962). The BWX is not as subject to anatomical limitations due to the hard palate as periapical radiographs and thus deviates little from parallelism (Reed and Polson 1984). The BWX is able to indicate "true" bone loss with a sensitivity of 1.00 , initially and after two incremental $1 \mathrm{~mm}$ bone reductions in cadavers (Hildebolt et al 1993).

BWXs are used to assess alveolar bone loss because they are relatively inexpensive and efficient and expose patients to less radiation (2-4 films) 
and discomfort than a full mouth PA series (16 - 18 radiographs). In addition, BWX are often required for periodic assessment of other dental conditions, such as caries and are therefore readily available for periodontal evaluation (Gjermo et al 1984).

Conventional BWX radiographs are considered adequate for periodontal disease surveys of adolescents with slight periodontitis as well as for older populations experiencing moderate to severe periodontitis (Blankenstein et al 1978; Selikowitz et al 1981; Gjermo et al 1984; Hansen et al 1984; Kallestal and Matsson 1991).

Measurement errors which may occur with the use of BWX are: improper film positioning, overlapping of teeth, incorrect vertical angulation of $x$-ray beam, double image, cone-cutting, improper processing and improper exposure. These errors however are not restricted to the BWX technique and are also evident with PAs and PANs (Nysether and Hansen 1983).

\section{PA's}

Radiographic techniques are designed to provide an accurate representation of the tooth and bone. However, when the validities of three techniques were compared in their ability to estimate the actual level of alveolar bone during periodontal flap surgery, it was found that PAs were significantly more accurate than BWXs or PANs. BWXs were significantly more accurate than PANs. There was an underestimation of bone loss of 11 - 23\% using BWX, 9 - 20\% with PAs and 13 - 33\% with PANs (Akesson et al 1992). PAs may be more accurate due to their more optimal intraoral film placement which results in less overlapping of approximal surfaces often evidenced on BWX and PANs. 
Radiographic survey results derived from PAs are not interchangeable with those from BWXs. In fact, their results are not well correlated with one another when the same site is assessed using the two different techniques. Differences may be due to variations in film placement, patient cooperation or to x-ray beam angulation. In assessments of crestal alveolar bone level, BWX measurements showed consistently greater loss than PAs in $94 \%$ of site comparisons. The results suggest that all studies should report the radiographic technique employed and that studies using different methods should be compared with caution (Reed and Polson 1984).

The routine use of a full-mouth survey of PAs has decreased due to concern about exposure to excess radiation and a reduction in the prevalence of rampant dental disease. Except in heavily-restored dentitions or where bone loss is $>5 \mathrm{~mm}$, the use of two BWX radiographs has been shown to provide similar diagnostic information as eight PA films of the premolar and molar teeth at about $25 \%$ the radiation exposure (Wall et al 1979).

\section{PAN}

A PAN imparts about $33 \%$ the radiation exposure of a full-mouth survey of PAs. It is also more convenient/comfortable for the patient and a less time-consuming procedure for the technician. The PAN technique is a satisfactory method for visualization of advanced bone loss but less so for slight bone loss (Akesson et al 1992). There is often a degradation of the image and a loss of fine detail due to the use of intensifying screens. In addition, critical landmarks such as the CEJ and $\mathrm{AC}$ are often obscured by overlapping teeth or lack sufficient detail for diagnosis (Wall et al 1979). Despite these limitations, direct measurement of the CEJ to AC on PANs 
has been shown to be an accurate and valid estimator of "true" bone loss when compared to direct bone measurement during periodontal surgery (Kaimenyi and Ashley 1988). Direct measurement of the CEJ to AC is a more valid method of bone level than using the proportion of tooth with bone support approach when employing PAN films (Kaimenyi and Ashley 1988).

\subsubsection{Reliability of Methods of Alveolar Bone Loss Measurement}

Various methods of estimating bone loss from radiographs have been used. Some express bone loss as a proportion of total length of the tooth or of the root length alone (Engelberger et al 1963; Bjorn and Holmberg 1966; Goodson et al 1984). Schei (1959) used a ruler designed to allow for a $1 \mathrm{~mm}$ 'normal' distance from the CEJ-AC to assess bone loss in terms of the proportion of the tooth normally surrounded by bone. Others have evaluated bone loss from radiographs and compared the distance from the CEJ-AC to intraoral measurements and found that the two measurements differed by $1.04 \mathrm{~mm}$ with radiographs giving a lower estimate of bone loss than the direct "open bone" measurements (Suomi et al 1968). Less difference (1.0 $\mathrm{mm}$ ) was found when examining dried mandibles (Stoner 1972). The latter study however was conducted among young individuals (8 - 17 years) in whom the prevalence and severity of periodontitis would have been minimal.

Alveolar bone level has also been quantified using fixed reference points such as the CEJ and the crest of the alveolar bone (AC). An engineers compass or a transparent ruler has been used to make the measurements. The compass has been used to evaluate the distance between the CEJ and AC on BWX, PAs and PANs (Kaimenyi and Ashley 1988; Akesson et al 1992). 


\section{Summary:}

Although radiographic evidence of alveolar bone loss has been shown to be associated with measurement errors, radiographs are able to objectively indicate a history of periodontitis.

Based on the review of the radiographic literature, a number of methodological decisions were made for the current study: 1) Radiographic evidence of $>3 \mathrm{~mm}$ bone loss would be indicative of a history of AP; 2 ) Partial mouth assessment of bone loss including molars and second premolars would be considered representative of the periodontal history of the entire dentition; 3) BWX radiographs would be used where available but in order to minimize the number of subjects that would be excluded for lack of BWX, PAs or PANs would be substituted. Records were made of the number of subjects who had their periodontal status determined using each method. Results are found in Chapter 8; 4) An engineers compass with a fixed reference points was used to evaluate each film for the presence of $>3$ $\mathrm{mm}$ bone loss.

\subsubsection{Probing Depth}

\section{Validity:}

Probing depth (PD) is the distance between the free gingival margin and the apical depth of the probe tip penetration (Caton 1993; Ismail and Lewis 1993). Probing depth has been shown to be variable in its occurrence with periodontitis and although it is hypothetically related (content validity) to the disease, it is not considered an objective measure of destructive periodontitis (Goodson 1990).

Measurement of attachment loss, based solely on PD, assumes that there is a direct relationship between PD measurements and the actual degree of attachment loss as represented by the area of root surface which 
has been denuded of periodontal ligament (Theil and Heany 1991). Studies have shown that this relationship does not always exist. In fact, a single PD measurement is at best, an indirect measure of an individual's history of periodontal disease (Goodson et al 1982) and may bear no relationship to current disease status or to future disease risk (Griffiths et al 1988) for reasons identified in the following sections.

\section{Related Measurement Errors:}

The criterion validity of $\mathrm{PD}$ to identify connective tissue attachment has been studied and reviewed extensively (Listgarten 1972; van den Velden 1978; van der Velden and DeVries 1978; Listgarten 1980; van der Velden 1982; Clerehugh and Lennon 1984; Griffiths et al 1988; Machtei et al 1992; Caton 1993.

$\mathrm{PD}$ as a measure of history of attachment level was investigated using denuded root surfaces of 236 teeth in 41 dried human skulls. Probe readings were not a precise measure of attachment level, particularly in areas of severe destruction (Theil and Heany 1991). However, lack of precision may have been due partially to use of a linear measurement tool i.e., the probe, to assess surface area attachment level.

Listgarten (1972) suggested that a distinction be made between "histological" and "clinical" pocket depth to differentiate between the depth of the actual anatomic defect and the measurement recorded by the probe. Studies suggest that probing depth is not a valid indicator of clinical attachment level because the probe may penetrate beyond the junctional epithelium and $0.3-0.5 \mathrm{~mm}$ into the underlying connective tissue, particularly in the presence of inflammation or increased probing pressure. Such penetration results in an overestimation of histologic PD and true CAL. In more healthy sulci and following periodontal treatment, 
the probe tip usually fails to reach the base of the junctional epithelium due to the so-called "long" junctional epithelium, thus underestimating CAL (van der Velden 1978; Listgarten 1980; Badersten et al 1984; Polson and Goodson 1985; Griffiths et al 1988).

Systematic measurement error may also be introduced by the use of improper probing sites or angulation as well as through inadequately calibrated/standardized probes and examiners or by rounding measurements up/down (van der Velden 1978; van der Velden and DeVries 1978; Listgarten 1980; Griffiths et al 1988). Such errors may systematically result in either an over or underestimation of periodontitis.

Other related measurement errors associated with PD include: Increased PD may result from edema or pseudopocketing, thus incorrectly labeling gingivitis as periodontitis; increased PD may be masked by concomitant gingival recession which tends to underestimate periodontitis (Machtei et al 1992).

Longitudinal studies using repeated-measurements of PD are necessary to identify disease activity, progress and susceptibility. Single, cross-sectional $\mathrm{PD}$ readings can, at best, indicate a history of periodontal disease (Griffiths et al 1988).

\section{Reliability:}

Reliability of PD measurements has been studied. Differences between duplicate recordings generally range from $2-3 \mathrm{~mm}$, depending on the examiners and on the periodontal condition. Deeper PD and proximal surface recordings are less reproducible as are surfaces involving inflammation (Badersten et al 1984). Inter-rater reliability of $95 \%$ is often only obtained when a wide range of PD $(2 \mathrm{~mm})$ is set (Ismail and Lewis 1993). However, PD may be reproduced within $+/-1.0 \mathrm{~mm}$ when pressure- 
sensitive probes are used with standardization stents (Badersten et al 1984). These latter methods may not be practical for large epidemiological studies.

\section{Summary:}

Although the validity and reliability of $\mathrm{PD}$ in the diagnosis of $\mathrm{AP}$ are suspect, a decision was made to incorporate existing records of $\mathrm{PD} \geq 5 \mathrm{~mm}$ and $P D \geq 7 \mathrm{~mm}$ in the current study, primarily due to unavailability of clinical attachment level measurements. Concordance of PD and other Diagnostic Criteria were assessed against the gold standard radiographic evidence of attachment loss. Results are reported in Chapter Six.

\subsubsection{Plaque Scores}

\section{Validity:}

Bacterial plaque has been closely associated with the prevalence and severity of periodontitis. To establish content and construct validity, any study of the epidemiology of periodontal disease, whether descriptive, analytic, or experimental, must take into account the presence/extent of plaque (Machtei et al 1992; Greene 1990).

Microbial plaque has been accepted as the primary etiologic agent in periodontal diseases (Polson and Goodson 1985). The microbial composition of plaque varies among individuals, among sites within the same individual, and the supragingival microflora is different from that found subgingivally in periodontally involved sites (Socransky 1977).

There is overwhelming evidence that the direct cause of gingivitis is the accumulation of microbial plaque on and near the cervical region of the teeth (Löe et al 1965; Page 1986). Most gingivitis lesions are transient or persistent and do not progress to periodontitis. However, since it is impossible to determine which lesions will progress, efforts are taken to 
control and prevent gingivitis. The prevention of gingivitis requires measuring, monitoring and controlling plaque.

Most measurements of supragingival plaque involve assessment of the tooth surface area covered by plaque and/or plaque thickness (Greene 1990). The validity of two predominant plaque score systems is discussed.

\section{The Plaque Index:}

The system which is most commonly used today for assessing plaque in clinical trials or experimental epidemiology is the Plaque Index (Silness and Löe 1964). This index has been used to assess plaque accumulation in a controlled clinical trial. The study demonstrated that withdrawal of all measures of oral hygiene resulted in gross accumulations of soft debris and the development of marginal gingivitis within 21 days in all twelve subjects. The criterion validity of the Plaque Index system to measure changes in plaque accumulation was evidenced when concurrent bacteriological examinations showed the number of microorganisms in the gingival area increased and that distinct changes in the relative composition of the flora occurred. Re-institution of oral hygiene resulted in healthy gingivae and re-establishment of the original bacterial flora (Clerehugh and Lennon 1984). The Plaque Index is useful for clinical trials and longitudinal studies because it is sensitive to small changes. However the index criteria are somewhat subjective and it requires well-trained and experienced examiners to obtain reliable data (Mandel 1974).

\section{The Plaque Control Record:}

Proportional plaque measurement indices have also been used in clinical and epidemiological studies (O'Leary et al 1972; Greene 1990). 
The O'Leary proportional plaque control record includes an assessment of the presence/absence of adherent, disclosed plaque at the gingival margin. Disclosing solution helps visualize plaque and, when used along with an explorer, differentiates between the organic pellicle (which is relatively free of bacteria) and bacterial plaque (Mandel 1974). The number of surfaces which have evidence of plaque serves as the numerator while the number of tooth surfaces present serves as the denominator, resulting in a percentage of available tooth surfaces with plaque (the plaque score). The validity of this index as an indicator of a history of AP has not been reported. However, it has been shown to be sensitive to small changes as it measures the presence versus absence of plaque. It may be more objective than the Plaque Index because the examiner does not rate the amount of plaque, just its presence/absence. Less subjectivity of an index is an advantage when multiple examiners are involved as in the current study. It must be recognized that one cross-sectional measure of plaque is not likely to be a valid indicator of lifetime experience with AP, but rather a measure of perhaps transient oral hygiene behavior and therefore an indicator of gingivitis.

\section{Reliability:}

Most clinical measurements of plaque focus on supragingival deposits since measurement of the quantity and quality of subgingival bacterial plaque is plagued by logistical problems related to the handling of anaerobic bacteria, and is clearly not feasible for large epidemiological studies. Supragingival quantitative measurements of plaque however are not intended to represent variations in the quantity or quality of subgingival 
plaque. The latter may be more relevant to clinical rather than epidemiological studies.

Assessing the reproducibility of plaque indices is difficult since the index generally involves the removal of a portion of the plaque as part of the scoring technique. As a result, second evaluations tend to show lower scores (Kingman et al 1991).

\section{Summary:}

Although the validity and reliability of plaque scores are suspect, information regarding oral hygiene and variables associated with it are essential in understanding periodontitis (Griffiths et al 1988). Results are reported in Chapter Eight. For the purposes of the current study, the O'Leary plaque control record was used because it was available from existing records.

\subsubsection{Bleeding on Probing}

\section{Validity:}

Gingivitis has been closely correlated with the prevalence and severity of periodontitis. To establish content and construct validity, any study of periodontal disease must take into account the presence/extent of gingivitis (Greene 1990; Machtei et al 1992). Gingivitis, may be a sign or symptom of periodontitis but it does not have criterion validity for measuring destructive periodontitis. Gingivitis is variable in its occurrence with the disease and it is primarily hypothetically related to periodontitis (Goodson 1990).

The validity of gingival indices to assess gingivitis has been studied. Indices of gingivitis typically focus on color, contour, bleeding, extent of involvement and crevicular fluid (Ciancio 1986). The Gingival Index (GI) 
(Löe and Silness, 1963) has been used to measure gingivitis in a longitudinal study of the effect of plaque accumulation on gingivae. Gingival conditions deteriorated over 21 days while no oral hygiene was practiced. After recommencement of cleansing and reduction in the Plaque Index, gingival inflammation resolved in approximately seven days. The GI appears to be a valid indicator of the presence of gingivitis and its association with plaque accumulation (Löe et al 1965).

Numerical indices such as the Gingival Index (GI) are simple, sensitive to small changes and they permit examiner calibration. They are limited however because they do not represent a continuous measure or a ratio scale i.e., it may be inappropriate to calculate mean GI scores from categorical measurements.

Indices which assess gingivitis based on the presence/absence of bleeding on probing (BOP) have, on the other hand, been shown to be objective and associated histologically with gingival inflammation (Greenstein et al 1981). Morphometric analysis of gingival biopsies support the detection of gingivitis based on bleeding following probing (Davenport et al 1982).

Repeated bleeding on probing at recall examinations during a two year longitudinal study was associated with an increased tendency to lose probing attachment (Badersten et al 1984). However, the ability of bleeding to predict attachment loss was less than $30 \%$ i.e., sites that bled at each recall had a $70 \%$ chance of not losing attachment. In other words, there may be an overestimation of predicted disease progression as well as overtreatment if bleeding alone is used as the predictor of disease activity.

It has been suggested that BOP may be one of the earliest and most reliable signs of gingival inflammation (Greestein et al 1981; Ciancio 1986). The presence/absence of bleeding following probing has been suggested for 
use in epidemiological studies, short term clinical trials and in the daily practice of dentistry (Ainamo and Bay 1975). However, it is essential that the probe reach the depth of the pocket in order to determine validly the bleeding status of the underlying tissues. Some bleeding indices stimulate and assess only superficial gingivae (Greenstein et al 1981; Engelberger 1983; Ciancio 1986).

\section{Reliability:}

The criteria used in the GI have been shown to be somewhat subjective and to require substantial examiner calibration. The GI is not the index of choice where multiple examiners are employed. The reliability of gingival bleeding in measuring gingivitis has been estimated to be $90 \%$ (Caton et al 1988).

It is known that shallow sulcular bleeding is influenced by the state of the marginal gingivae which, like supragingival plaque, is transient (Griffiths et al 1988). The transient nature of plaque and gingivitis may partially explain the poor predictability of bleeding on probing for periodontitis. It has been suggested that use of repeated measurements in longitudinal studies may be more appropriate than a single cross-sectional measure of bleeding to predict disease progression (Griffiths et al 1988).

The reliability of bleeding indices is difficult to establish since repeated probing of the site within 90 minutes has been shown to produce higher readings due to tissue stimulation from the initial probing (Janssen and Van Palenstein-Halderman 1985). Reliability of bleeding indices has been enhanced when controlled pressure-sensitive probes were used (Polson et al 1980). However, the latter may not be practical for large epidemiological studies. 


\section{Summary:}

Although the validity and reliability of BOP in relation to a history of AP are somewhat suspect, information regarding gingivitis and variables associated with it are essential to understanding periodontitis. Existing BOP scores were analyzed in the current study. Results are reported in Chapter Eight.

\subsection{SECONDARY DATA FROM MEDICAL HISTORY Validity:}

The validity of a number of the questions in the medical history section of the dental chart was uncertain. However, two questions appeared to have face validity (Q12, Q22) (hypertension and diabetes) (Appendix 6b). The medical histories were completed as face-to-face interviews between student clinician and patient. As a result, the atmosphere was conducive to answering questions; that is, questions could be explained and optional follow-up probing questions could be asked. The patients were probably quite motivated to provide accurate information to the interviewer since this was part of their clinical needs assessment and treatment planning session.

The validity of certain questions dealing with socially sensitive issues such as smoking have generally been shown to be under-reported (Schlesselman 1982). However, the clinician-interviewers were unaware of the patients' dental health at the time of the medical history so interviewer bias was not an issue. In addition, there is no reason to believe that those with periodontitis (cases) would respond with more/less information bias than those without periodontitis (controls).

A number of questions in the chart's medical history were believed to be inadequate for the purposes of the current study, for example Q45, "Do 
you smoke, drink or use non-prescription drugs?" This question dilutes the emphasis given to smoking, a key study variable; Q1, "Do you have regular dental appointments"? "Regularity" is not operationally defined.

\section{Reliability:}

No effort was made to determine the reliability of the medical history data obtained from the charts since this was a secondary source of data. However, for key questions, the reliability of responses was assessed by comparing the responses to the responses obtained from the telephone interview. Since some questions were not posed in the same manner, reliability often was impossible to determine. The reliability of clinical blood pressure readings was not assessed. It appeared that the measurement was made only once. It is uncertain whether the measurement was taken at a time in the appointment when the patient was most at ease. It is also unknown whether clinical instructors were able to reproduce the readings.

\section{Summary:}

The validity and reliability of the secondary medical history is uncertain. It was for this reason that the telephone interview was designed. An attempt was made to assess the reliability of the telephone interview with the secondary data where it was possible. Results are reported in Chapter Eight.

\subsection{THE TELEPHONE INTERVIEW}

Telephone interviews have been shown to stimulate and maintain respondents' interest and to create an atmosphere conducive to answering questions (Abramson 1990). Questions may be repeated, and if necessary (and in accordance with training guidelines), a prompting explanation 
provided. Suitable standardized follow-up probes/prompts were printed on each interview form.

The response rate (93.4\%) was enhanced by sending a pre-interview letter of information from the Dean of the Faculty of Dentistry to all potential subjects in which the intent of the study was outlined and encouragement for voluntary participation stressed (Appendix 6a). High response rates improve the external validity of a study.

Telephone interviews (Appendix 6e) were utilized rather than inperson interviews due to reduced cost and increased convenience. Mail surveys were not used because of an anticipated compromised response rate and the inability to clarify questions with potential respondents. Telephone surveys have been shown to result is less concealment of socially undesirable behavior than in-person interviews (Pless and Miller 1979).

Telephone interviewers were blind to the periodontal status (case/control) of the respondents in an effort to avoid interviewer bias.

\section{Validity:}

Questionnaire Validity - The content validity of the telephone interview was emphasized to ensure that all items were relevant to the concept being studied. Content validity is generally tested by collaboration with experts in the field (McDowell and Newel 1987).

The wording, sequencing, and logical flow of the questions were evaluated in several ways while drafting the questionnaire: The questions were shown to experts in survey design and epidemiology as well as to physicians, periodontists, and other related health professionals and ultimately to a biostatician and data entry specialist. Revisions were made to the questionnaire and subsequently, the questionnaire was piloted inperson among four patients at the Faculty in an attempt to obtain direct 
reactions to questions and respondent suggestions for clarification of the questions. Following further revisions, the interview was pre-tested among nine individuals who were active patients at the Faculty of Dentistry, but who were not potential study participants. An attempt was made to include respondents of a variety of age, periodontal status, English language skills, and gender. Each of the three interviewers conducted three telephone interviews while the other two interviewers 'listened-in' through a conference call mechanism. All interviewers independently scored the responses. Subsequently, sets of responses for each of the questions were assessed. Any question which failed to show uniform responses was modified slightly to enhance clarity. Modifications involved the addition of prompts and clearly indicating the number of cigarettes per package.

- Face Validity: All questions had face validity, except Q19. The question's intent to measure degree of life-stress was not accomplished due to inadequate wording. Respondents tended to give the average "moderate" stress response as discussed in Chapter Eight.

- Criterion: The criterion validity of question 6 was tested by comparing the subject's telephone response to the medical history's clinical blood pressure reading $(\mathrm{SBP}>140$ or $\mathrm{DBP}>90 \mathrm{~mm} / \mathrm{Hg}$ ). Similar testing was done for age, gender, and history of diabetes. Results are reported in Chapter Eight.

- Construct: Correlation between a number of questions was tested to determine construct validity. For example, lower income was positively correlated with lower education, greater frequency of 
brushing was correlated with regular dental visiting. Results are reported in Chapter Eight.

- Content: The questions which assessed Socio-economic status (\#2327) have been shown previously to have content validity. Results are reported in Chapter Eight.

\section{Reliability:}

Reliability of data obtained from telephone questionnaires regarding risk-exposure is influenced by recall bias, social acceptability bias as well as errors in classification and difficulties in accurately assessing confounding variables.

Reliability of key questions on the interview was assessed when possible by comparing interview responses with those obtained from subjects' existing chart medical/dental history. Inter/intra-rater reliability of the telephone interview was assessed using Kappa statistics and Intra Class Correlation Coefficients. Results are reported in Chapter Eight. 


\section{PART III}

\section{RESULTS AND CONCLUSIONS}




\section{CHAPTER EIGHT}

\section{ANALYSIS AND RESULTS}

\subsection{OBJECTIVES OF ANALYSES}

The objectives of the analysis were to:

1. describe the distribution of the explanatory study variables within the study population using descriptive statistics such as measures of central tendencies and dispersion;

2. test hypotheses about the existence or strength of relationship between pairs of study variables using bivariate statistics;

2a. Hypothesis: There is a significant association between a history of AP and antecedent exposure to specific health compromising behaviors, medications, chronic diseases/conditions, sociodemographic and certain oral health variables;

3. determine the most appropriate diagnostic criterion for case identification based on the criterion's sensitivity, specificity and Kappa statistic;

4. compare exposure to explanatory variables among individuals with a history of periodontitis to those without using bivariate statistics such as the t-test and Chi-square test;

5. test hypotheses about the relationships between two or more study variables while controlling for other variables, using multivariate statistics, namely, multiple logistic regression;

5a. Hypothesis: There is a significant increased risk for AP among those with antecedent exposure to the study variables. 


\subsection{DATA ENTRY}

Prior to data entry, all telephone questionnaires were placed in numerical order by chart number. Data were subsequently entered into a computer by an experienced data entry clerk using Data Entry Emulator software package (Computer Keyes 1991). The accuracy of data entry was verified through a double-entry process which automatically notified the clerk when differences occurred. Where data points differed, $(n=3)$, they were re-entered directly by the clerk from the original survey.

\subsection{SCREENING OF DATA}

Clinical data from dental charts previously entered by the PI into Lotus 2.0 spreadsheet software (LeBlond and Cobb, 1985) was imported along with data from telephone interviews into NCSS (Number Cruncher Statistical System Product 5.3; Hintze 1992). All data were subsequently screened for appropriate range of values.

\subsection{THE STUDY POPULATION}

The distribution of cases and controls among the target population is described by subjects' year of periodontal examination (Table 8.1). The "control" target population included all $(\mathrm{n}=205)$ individuals who were classified, "01" or "02" at the time of their examination. Among the "case" target population, all individuals classified, "04" $(\mathrm{n}=81)$ during the study period were included, representing the most severely diseased. In addition, a random selection of all individuals $(\mathrm{n}=351)$ classified, "03" was made $(\mathrm{n}=$ 124). The percentage of class 03's by year of exam for selected subjects was consistent with the total case " 03 " target population. The sampling frame population was $205+81+351=637$. Selection was done in the manner described to achieve balance and to ensure inclusion of the most severe 
cases thus maximizing the discriminating power of the analyses. Both groups initially consisted of 205 subjects however, one case was excluded when it was found that the financial account had been sent to collection. It was thought that the responses may be influenced by prevarcation bias or that the individual may not be representative of the target population.

TABLE 8.1 Distribution of cases and controls among target and study population by year of exam

\begin{tabular}{|c|c|c|c|c|c|c|c|c|c|c|}
\hline \multicolumn{5}{|c|}{$\begin{array}{c}\text { Control study population } \\
\text { vs } \\
\text { Control participants } \\
\end{array}$} & \multicolumn{6}{|c|}{$\begin{array}{c}\text { Case study population } \\
\text { vs } \\
\text { Case participants } \\
\end{array}$} \\
\hline \multicolumn{5}{|c|}{ Controls by year of exam } & \multicolumn{6}{|c|}{ Cases by year of exam } \\
\hline \multicolumn{3}{|c|}{$\begin{array}{l}\text { Control target } \\
\text { population }\end{array}$} & \multicolumn{2}{|c|}{$\begin{array}{c}\text { Control } \\
\text { participants }\end{array}$} & \multicolumn{2}{|c|}{$\begin{array}{c}\text { Case target } \\
\text { population } \\
\text { (03's) }\end{array}$} & \multicolumn{2}{|c|}{$\begin{array}{l}\text { Case study } \\
\text { population }\end{array}$} & \multicolumn{2}{|c|}{$\begin{array}{c}\text { Case } \\
\text { participants }\end{array}$} \\
\hline & $\#$ & $\%$ & $\#$ & $\%$ & $\#$ & $\%$ & $\#$ & $\%$ & $\#$ & $\%$ \\
\hline 1991 & 58 & 28.0 & 53 & 27.9 & 86 & 24.5 & $\begin{array}{c}35(03)+ \\
18(04)=53\end{array}$ & 25.9 & 47 & 24.5 \\
\hline 1992 & 43 & 21.0 & 38 & 20.0 & 81 & 23.1 & $\begin{array}{c}28(03)+ \\
22(04)=50\end{array}$ & 24.5 & 47 & 24.5 \\
\hline 1993 & 56 & 27.0 & 52 & 27.4 & 93 & 26.5 & $\begin{array}{c}30(03)+ \\
24(04)=54\end{array}$ & 26.5 & 52 & 27.0 \\
\hline 1994 & 48 & 23.0 & 47 & 24.7 & 91 & 25.9 & $\begin{array}{c}30(03)+ \\
17(04)=47\end{array}$ & 23.1 & 46 & 24.0 \\
\hline \multicolumn{3}{|c|}{$\mathrm{n}=205$} & \multicolumn{2}{|c|}{$\mathrm{n}=190$} & \multicolumn{2}{|c|}{$\mathrm{n}=351$} & \multicolumn{2}{|c|}{$\begin{array}{c}\mathrm{n}=123(03) 81 \\
(04), \mathrm{n}=204\end{array}$} & \multicolumn{2}{|c|}{$\mathrm{n}=192$} \\
\hline
\end{tabular}

\subsection{PARTICIPANTS VERSUS NONPARTICIPANTS}

During the period November 1994 - January 1995, a total of four hundred nine (409) individuals were invited to participate in the study (study population). Of these, $382(93.4 \%)$ completed the telephone interview. Ten $(2.4 \%)$ individuals could not be contacted by telephone or mail and seventeen $(4.2 \%)$ refused to participate. 
TABLE 8.2 Response rate to telephone interview

\begin{tabular}{lll}
\hline Eligible for telephone interview & $\#$ & $\%$ \\
Interview completed & 409 & $(100)$ \\
Refused participation & 382 & $(93.4)$ \\
Unable to be contacted & 17 & $(4.2)$ \\
\hline
\end{tabular}

The number of interviews completed per interviewer and the response rate according to interviewer are presented in Table 8.3. As planned, the PI completed the majority of the interviews (75.4\%). Inter and intra-examiner reliability are discussed in Section 8.6.3.

TABLE 8.3 Response rate to telephone interviews by interviewers

\begin{tabular}{cccccccc}
\hline Interviewer & Number (\%) completed & Number (\%) refused & $\begin{array}{c}\text { Number (\%) not } \\
\text { contacted }\end{array}$ \\
\hline$\# 1(\mathrm{~K})$ & 55 & $55 / 382$ & $(14.4)$ & 2 & $2 / 55$ & $(3.64)$ & 4 \\
$\# 2(\mathrm{C})$ & 39 & $39 / 382$ & $(10.2)$ & 9 & $9 / 39$ & $(23.08)$ & 4 \\
$\# 3(\mathrm{~S})$ & 288 & $288 / 382$ & $(75.4)$ & 6 & $6 / 288$ & $(2.08)$ & 2 \\
TOTAL & 382 & & $(100)$ & 17 & $17 / 382$ & & 10 \\
\hline
\end{tabular}

The total number of interviews completed by each interviewer. The total number of refusals along with the \% refusals per interviewer. Total number of not contacted by each interviewer.

Reasons given for non-participation in the telephone interview were documented and reported in Table 8.4. The primary response was, "no reason" ( $=5)$ followed by, "not interested" $(n=3)$. 
TABLE 8.4 Reasons given for not participating in telephone interview

\begin{tabular}{lc}
\hline No reason given & 5 \\
Language problem & 1 \\
Dissatisfaction with Faculty of Dentistry & 2 \\
Not interested & 3 \\
Didn't have time & 2 \\
Against philosophy & 2 \\
Subject ill & $\underline{2}$ \\
TOTAL & $\mathbf{1 7}$ \\
\hline
\end{tabular}

The study attempted to determine whether there were any differences between participants and non-participants regarding their level of education. However, due to the few number of non-participants $(n=17)$ willing to provide education-related information $(n=8)$, differences could not be analyzed adequately.

Participants in the telephone interview were compared to nonparticipants regarding their periodontal status (case vs control) and the date of their periodontal examination. As seen in Table 8.1, participation by year appeared to be consistent. Since the rate of participation in the study was adequate (93.4\%), it was felt that generalization of results to the target population was valid.

\subsection{RELIABILITY, VALIDITY AND QUALITY OF DATA}

\subsubsection{Intra-Rater Reliability: Radiographic Interpretation}

Intra-examiner reliability of the dentist's radiographic interpretation was determined by having the dentist unknowingly re-read a random selection of radiographs two days after their first reading. Reliability was assessed for BWX radiographs $(n=15$ sets) as well as for PA/PAN radiographs ( $\mathrm{n}=8$ sets). 
Intra-examiner reliability was calculated by:

$\%$ Agreement $=\frac{\text { \# classified twice as cases }+ \text { \# classified twice as controls }}{\text { total \# examined twice }} \times 100$

(Fleiss 1981)

The percent agreement for the BWX was $80.0 \%$ and $87.5 \%$ for the PA/PAN radiographs.

The following Kappa coefficient statistic was used to more critically quantify intra-rater reliability by eliminating any agreement that was due to chance alone:

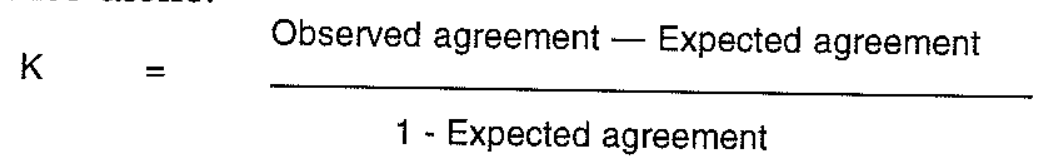

(Fleiss 1981)

When two measurements agree only at the chance level the value of Kappa is zero and when the values agree perfectly, the Kappa is 1. Kappa values $<0.4$ indicate poor reliability while values $0.4-0.75$ represent fair to good reliability, i.e., beyond chance and $>0.75$ displays excellent reliability (Kelsey et al 1986; Fleiss 1981)

The reliability of the dentist to label films either as case or control twice was as follows:
BWX
$\mathrm{K}=0.59$
PA/PAN
$\mathrm{K}=0.75$

\subsubsection{Reliability of PA/PAN Radiographs Compared to BWX Radiographs}

Where subjects had no BWX, PA/PAN radiograph were used to determine presence of alveolar bone loss. Seventy five percent of the initial study population had BWX available. Further details regarding types of radiograph used are reported in Table 8.5. 
TABLE 8.5 Radiograph types used to determine case/control status

$$
(n=409)
$$

\begin{tabular}{lcc}
\hline \multicolumn{1}{c}{ Type } & $\#$ & $\%$ \\
\hline Only BWX & 300 & 75 \\
PA & 72 & 18 \\
PAN & 28 & 7 \\
No radiographs available & 9 & - \\
TOTAL SUBJECTS & $\mathbf{4 0 9}$ & \\
\hline
\end{tabular}

To determine the percent agreement of the PA/PAN technique in reproducing the same case/control diagnosis as BWX, thirteen subjects' PA/PAN radiographs were read one day subsequent to the reading of his/her BWX radiographs. The first thirteen randomly selected subjects who had both types of radiographs available were included. The dentist was unaware that alternate radiographs of the subject had previously been read. The percent agreement of the two techniques was $92.0 \%$, while the Kappa $=0.83$.

\subsubsection{Intra-and Inter-Examiner Reliability}

\section{Telephone Interview:}

Interviewer reliability was assessed by re-interviewing a random selection of forty $(n=40)$ subjects two weeks subsequent to their initial interview. Table 8.5a outlines the number of initial and re-interviews completed by each interviewer. 
TABLE 8.5a Re-Interview Schedule

\begin{tabular}{ccccc}
\hline Interviewer & \# Completed & \#Refused & \#No Contact & Re-Interview \\
\hline $1(\mathrm{~K})$ & 55 & 2 & 4 & 5 of \#3 (S) \\
$2(\mathrm{C})$ & 39 & 9 & 4 & 5 of \#3 (S) \\
$3(\mathrm{~S})$ & 288 & 6 & 2 & 20 of \#3 (S) \\
& & & & 5 of \#1 (K) \\
TOTAL & $\mathbf{3 8 2}$ & $\mathbf{1 7}$ & $\mathbf{1 0}$ & $\mathbf{5}$ of \#2 (C) \\
\hline
\end{tabular}

The test-retest reliability and inter-examiner reliability of several key variables were assessed for categorical variables: gender, marital status, level of education using Kappa statistic while Intraclass Correlation Coefficient (ICC) was calculated for continuous variables: age, number of cigarettes smoked/day, number of years smoked (Shrout and Fleiss 1979).

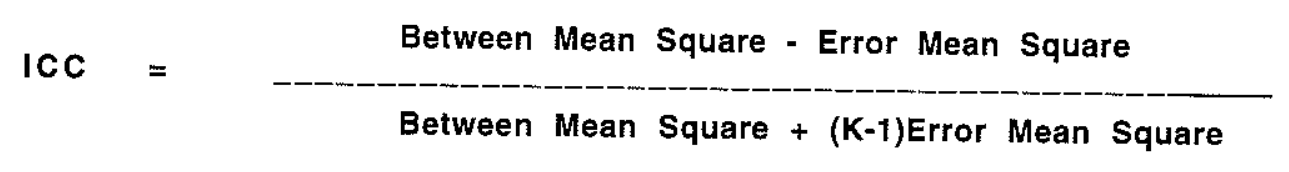

(Shrout and Fleiss, 1979)

TABLE 8.5b Test - re-test reliability (Intra examiner)

\begin{tabular}{lcc}
\hline \multicolumn{1}{c}{ Variable } & Reliability & KAPPA/ICC \\
\hline Age (years) & $100 \%$ & 1.0 \\
Gender & $100 \%$ & 1.0 \\
Marital Status & $100 \%$ & 1.0 \\
Education & $100 \%$ & 1.0 \\
\#Cig/day & $90 \%$ & 1.0 \\
\#Years smoked & $75 \%$ & 0.54 \\
\hline
\end{tabular}


TABLE 8.5 c Inter-examiner reliability

\begin{tabular}{lcc}
\hline \multicolumn{1}{c}{ Variable } & Reliability & KAPPA/ICC \\
\hline Age (years) & & \\
Gender & $100 \%$ & 1.0 \\
Marital Status & $100 \%$ & 1.0 \\
Education & $100 \%$ & 1.0 \\
\#Cig/Day & $95 \%$ & 0.9 \\
\#Years Smoked & $90 \%$ & 0.4 \\
\hline
\end{tabular}

Intra and inter-interviewer reliability was generally high except for variables, number of cig/day and number of years smoked. Since these variables were subsequently combined into number of cigarette-index (number of cig/day $\mathrm{x}$ \# years smoked), and that variable further categorized into $0 ; 1-300 ; 301-500$; and > 500, reliability likely would have increased to an acceptable level.

\subsubsection{Validation and Comparison of Telephone Survey and Dental/Medical Chart Data:}

To determine whether data obtained from the telephone interview differed significantly from data obtained from the dental/medical records, a comparison was made of available relevant demographic and medical data including Age (date of birth), history of Hypertension, or Diabetes.

When date of birth given at the history and examination was compared with date of birth given at interview, all dates of birth were exactly the same except in three individuals where the date differed by one year (e.g. 1918 vs 1919).

The overall agreement between methods regarding a history of diabetes was $99 \%$ with a $\mathrm{K}=0.90$. The Kappa was 1.0 when those indicating "borderline" status were categorized as not having a history of diabetes.

The overall agreement between methods regarding a history of hypertension was $87 \%, \mathrm{~K}=0.63$. One explanation for a higher prevalence of 
hypertension reported in the telephone interview than in the existing medical chart could be that at the time of the medical/dental history, subjects had not yet had their blood pressure measured. As a result, many may have been unaware that their blood pressure was high. Students generally take the blood pressure after the verbal medical history has been recorded. Blood pressure measurements are then recorded in the chart and if high, the patient is told to visit his/her physician. Since the telephone interview asked, "Prior to $19 \ldots$, did a physician or nurse ever tell you that you had hypertension or high blood pressure?", the subject may have reported the reading taken at their first dental/medical examination at the Faculty. The results are reported in Table 8.6.

\section{TABLE 8.6 Validity of Interview}

\section{Variable}
I. $\quad$ AGE $(\mathrm{n}=382)$
(DOB) 379 had same DOB at interview as in Medical Record 3 differed by one year

II. DIABETES $(n=382)$

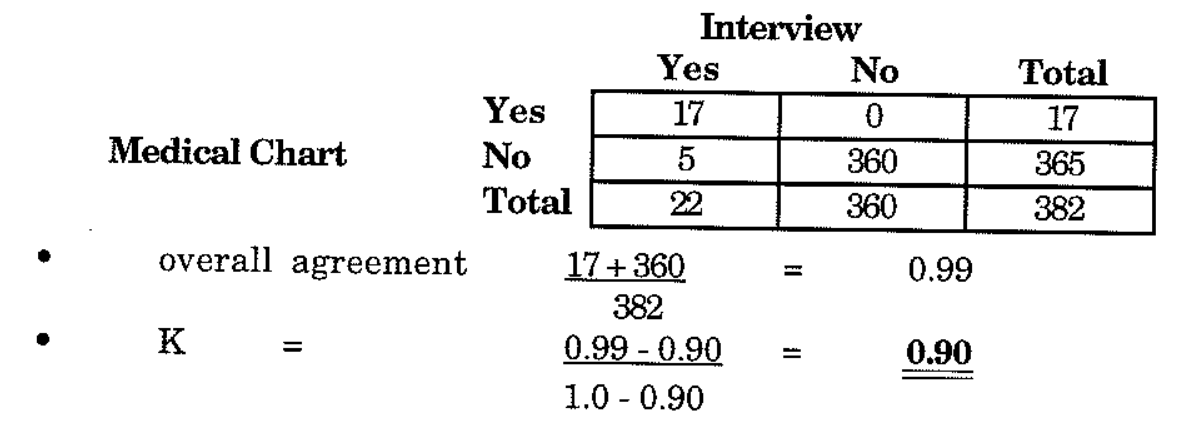

III. HYPERTENSION $(\mathrm{n}=380)$

\begin{tabular}{|c|c|c|c|c|c|}
\hline \multirow{5}{*}{ Medical Chart } & \multirow{5}{*}{$\begin{array}{l}\text { Yes } \\
\text { No } \\
\text { Total }\end{array}$} & \multicolumn{2}{|c|}{ Interview } & \multirow[b]{2}{*}{ Total } & \multirow[b]{5}{*}{$\begin{array}{c}2 \text { missing charts } \\
\text { values }=392\end{array}$} \\
\hline & & \multirow{2}{*}{$\begin{array}{c}\text { Yes } \\
62 \\
\end{array}$} & \multirow{2}{*}{$\frac{\text { No }}{13}$} & & \\
\hline & & & & 75 & \\
\hline & & 35 & 270 & 305 & \\
\hline & & 97 & 283 & 380 & \\
\hline overall agr & & $=$ & 0.87 & & \\
\hline $\mathrm{K}$ & & $=$ & $\underline{0.63}$ & & \\
\hline
\end{tabular}


The validity of the telephone interview was adequate among those variables assessed. The validity of other variables could not be established in the current study (e.g. smoking history).

\subsubsection{Quality of Interview:}

The interviewer's impression of the quality of the interview was documented immediately following each interview. Interviewers were asked to indicate their estimation of 1) the respondent's understanding of the questions; 2) the respondent's recall of information; and 3) the chances of getting the same responses in two weeks. It was determined a priori that subjects' questionnaires rated below "3" (average) would be excluded from analysis. No scores below "3" were reported. The "quality" of the interview, albeit somewhat subjectively determined was adequate. Results are reported in Table 8.7.

TABLE 8.7 "Quality" of interview $(\mathrm{n}=382)$

\begin{tabular}{|c|c|c|c|c|c|c|c|c|c|c|c|}
\hline \multicolumn{7}{|c|}{ Low } & \multicolumn{5}{|c|}{ High } \\
\hline & \multicolumn{2}{|c|}{1} & \multicolumn{2}{|c|}{2} & \multicolumn{2}{|c|}{3} & \multicolumn{2}{|c|}{4} & \multicolumn{2}{|c|}{5} & \multirow{2}{*}{ TOTAL } \\
\hline & $\#$ & $\%$ & $\#$ & $\%$ & \# & $\%$ & $\#$ & $\%$ & \# & $\%$ & \\
\hline $\begin{array}{l}\text { Respondent } \\
\text { understanding }\end{array}$ & 0 & 0 & 0 & 0 & 4 & 1.10 & 30 & 7.80 & 348 & 91.10 & 382 \\
\hline Recall & 0 & 0 & 0 & 0 & 5 & 1.31 & 29 & 7.59 & 348 & 91.10 & 382 \\
\hline $\begin{array}{l}\text { Chance getting } \\
\text { same response }\end{array}$ & 0 & 0 & 0 & 0 & 1 & 0.26 & 19 & 4.97 & 362 & 94.77 & 382 \\
\hline
\end{tabular}

Post-interview rating of "quality" of the interview by interviewers regarding respondent's understanding of questions, his/her recall of information, and likelihood of obtaining same response from the subject in two weeks.

\subsection{UNIVARIATE AND BIVARIATE ANALYSES}

Univariate and bivariate methods included histograms, normal probability plots, frequency distribution tables, skewness, kurtosis, crosstabulations, t-tests, analysis of variance, squared correlation coefficients, and simple linear or logistic regression analyses. These methods were used 
to assess the distribution of observations, to confirm the appropriateness of normality assumptions, to determine the relationships between variables, to detect collinearity between variables, and to identify the variables most suitable for multivariate analyses.

\subsubsection{Overview of Continuous Variables}

The distribution of continuous variables is outlined and missing values identified in Table 8.8. The mean age for participants at the time of their periodontal exam was 55.2 years $(\mathrm{SD}=12.7)$. The average plaque score for participants was $52.2 \%(\mathrm{SD}=26.9)$ while their average systolic and diastolic blood pressure was $129.6 \mathrm{~mm} / \mathrm{Hg}(\mathrm{SD}=16.4)$ and $81.9 \mathrm{~mm} / \mathrm{Hg}(\mathrm{SD}=$ $9.8)$ respectively. Smokers, on average had smoked for 18.5 years $(\mathrm{SD}=13.2)$ (Table 8.8).

\section{Missing Data:}

The pattern of missing data is believed to be more important than the amount of missing data since systematically missing values may affect generalizability of results. If few data points are missing in a random pattern in a relatively large sample, there is less concern than if there are many data points missing from a small-to-moderate-sized study. There are no established guidelines regarding the critical number of missing data points that may be tolerated (Tabachnick and Fidell 1989).

In the current study, missing values were relatively infrequent. Some data for approximately $7 \%$ of subjects were missing for variables: Plaque Score (PS) $(\mathrm{n}=32)$, Bleeding Score (BOP) $(\mathrm{n}=26)$, Systolic and Diastolic Blood Pressure (S/DBP) $(\mathrm{n}=32)$ as these data were unavailable from existing dental/medical records. 
Missing Plaque Scores (PS) were randomly distributed among all age groups, both genders and levels of education. However, there were more missing plaque scores among cases $(n=22)$ than controls $(n=10)$ and there was a trend for more missing Plaque Scores when subjects had more teeth present i.e., there were 20 missing scores among those with $\geq 20$ teeth present and only four missing among those who had fewer than 10 teeth present. There were more scores missing among those who brushed $\geq$ $1 \mathrm{x} /$ day $(\mathrm{n}=14)$ than those who didn't $(\mathrm{n}=2)$. The reason for missing PS may be that clinicians had a tendency not to calculate it among patients in whom the oral hygiene appeared excellent or in advanced cases where plaque was generalized.

Missing Bleeding on Probing scores were also randomly distributed among all age groups, both genders and levels of education. However, there were more missing BOP scores for controls $(n=24)$ than cases $(n=2)$ which may be explained by failure of clinicians to calculate a BOP score when there is no clinical evidence of inflammation. There was a tendency for more missing BOP scores when there were more teeth present that is, 18 were missing among those who had most $(\geq 20)$ of their teeth while only four were missing when the subjects had fewer than 10 teeth present and another four were missing among those with $10-19$ present. This situation suggests that BOP scores were taken less often on periodontally healthy individuals. There were more missing BOP scores among those who brushed $\geq 1 /$ day $(n=22)$ than those who brushed $<1 /$ day $(n=4)$. Clinicians may have been more likely to take the BOP in the presence of plaque and inflammation than when they were absent.

Since the missing data appeared generally to occur within a random subsample of the study population, it was appropriate to exclude individuals with missing values from analyses. 


\section{Outliers:}

Outliers (observations differing widely from the rest of the data) (Last, 1988), were identified through the use of histograms and normal probability plots. Outliers were observed in the following continuous variables: a) Number of Cigarettes Smoked/Day. This variable was logtransformed, yielding no outliers; b) Number of Teeth Present. A logtransformation of this variable did not take care of the outlier therefore, the variable was transformed into a categorical variable.

\section{Normality:}

Normality of distribution was evaluated using the Martinez and Iglewicz Normality Test (Hintze 1992) along with frequency histograms and normal probability plots. The critical value of $5 \%$ was set. Non-normality was said to exist when the test value was larger than the rejection value. The results are reported in Table 8.8.

Any variable which deviated from normality was log-transformed or a square root transformation attempted and the median value recorded. In the case of variables such as Bleeding on Probing, where the value is a percentage, a sine transformation was attempted. If these transformations did not achieve normality, the variable was categorized.

Deviations from normality were detected in variables: a) Bleeding Score. Sine transformation did not eliminate non-normality; therefore, the variable was transformed into a categorical variable.; b) Number of teeth = 5, 6, $7 \mathrm{~mm}$ PD. Neither a $\log$ nor square root transformation would eliminate non-normality so the variable was categorized.

\section{Skewness and Kurtosis:}

Skewness (how symmetrical the values were about the mean) was assessed along with Kurtosis (the flatness of the distribution) using the $\mathrm{Z}$ 
TABLE 8.8 Descriptive statistics of continuous variables

\begin{tabular}{|c|c|c|c|c|c|c|}
\hline Variable & $\begin{array}{l}\text { Mean (SD) } \\
\text { median }\end{array}$ & Range & $\begin{array}{l}\text { Sample } \\
\text { size }\end{array}$ & $\begin{array}{l}\text { Missing } \\
\text { values }\end{array}$ & $\underset{\text { (histogram) }}{\text { Outliers }}$ & $\begin{array}{c}\begin{array}{c}\text { Normality Martinez } \\
\text { \& Iglewicz test (mi) } \\
\text { alpha }=.05\end{array} \\
\end{array}$ \\
\hline $\begin{array}{l}\text { Sociodemographics Factors: } \\
\text { Age at H\&E (years) }\end{array}$ & $55.2(12.7)$ & $35-87$ & 382 & 0 & No & NS \\
\hline \multicolumn{7}{|l|}{ Behavior: } \\
\hline Number of cigarettes/day & $17.0(14.1)$ & $1-100$ & 382 & 0 & Yes & NS \\
\hline $\begin{array}{l}\text { Number of cigarettes/ day } \\
\text { logged }\end{array}$ & 2.64 & $.256-4.61$ & 382 & 0 & $\begin{array}{l}\text { Logged } \\
\text { yielded no } \\
\text { outliers }\end{array}$ & NS \\
\hline $\begin{array}{l}\text { Number of years smoked } \\
\text { Number of years smoked } \\
\text { sqr }\end{array}$ & $\begin{array}{c}18.9(13.2) \\
\quad 4.24\end{array}$ & $\begin{array}{c}1-51 \\
1-7.14\end{array}$ & $\begin{array}{l}382 \\
382\end{array}$ & $\begin{array}{l}0 \\
0\end{array}$ & $\begin{array}{l}\text { No } \\
\text { No }\end{array}$ & $\begin{array}{l}\text { NS } \\
\text { NS }\end{array}$ \\
\hline $\begin{array}{l}\text { Number cigarette years (\# } \\
\text { cig day } x \text { \# year smoke) }\end{array}$ & 375.97 & $1-4500$ & 382 & 0 & Yes & $M-I=2.40$ \\
\hline \multicolumn{7}{|l|}{ Oral findings: } \\
\hline Plaque score & $52.2(26.9)$ & $09-100 \%$ & 382 & 32 & No & NS \\
\hline Plaque score SIN & -0.262 & $-0.99-0.99$ & 382 & 32 & No & NS \\
\hline Bleeding score & $33.2(22.8)$ & $0-100 \%$ & 382 & 26 & No & $M-I=1.09$ \\
\hline Bleeding score SIN & -0.262 & $-0.99 \times 0.99$ & 382 & 26 & No & $\mathrm{M}-\mathrm{I}=0.13$ \\
\hline Number of teeth & $22.3(6.1)$ & $1-28$ & 382 & 0 & Yes & $\mathrm{M}-\mathrm{I}=1.73$ \\
\hline \multicolumn{7}{|l|}{ Medical health variables: } \\
\hline Systolic blood pressure & $129.6(16.4)$ & $94-180$ & 382 & 24 & No & $M-I=1.03$ \\
\hline Diastolic blood pressure & $81.9(9.8)$ & $52-128$ & 382 & 24 & No & $\mathrm{M}-\mathrm{I}=1.15$ \\
\hline
\end{tabular}


TABLE 8.8a Descriptive statistics of continuous variables (cont'd)

\begin{tabular}{|c|c|c|}
\hline Variable & Skewness & Kurtosis \\
\hline $\begin{array}{l}\text { Sociodemographic Factors: } \\
\text { Age at } \mathrm{H} \& \mathrm{E} \text { (years) }\end{array}$ & $\mathrm{Z}=0.97, \mathrm{p}=0.33$ & $\begin{array}{l}Z=-12.55, p<0.001 \text { (Not logged because Kurtosis is not believed to } \\
\text { be a concern when the variable is normally distributed and no } \\
\text { skewness exists.) }\end{array}$ \\
\hline $\begin{array}{l}\text { Behavior: } \\
\text { Number of cigarettes/ day }\end{array}$ & $\mathrm{Z}=8.93, \mathrm{p}<0.001$ & $\mathrm{Z}=7.01, \mathrm{p}<0.001$ \\
\hline $\begin{array}{l}\text { Number of cigarettes/ day } \\
\text { logged }\end{array}$ & $\mathrm{Z}=-3.64, \mathrm{p}<0.0003$ & $\begin{array}{l}\mathrm{Z}=0.07, \mathrm{P}=0.95 \text { (number of cigarettes per day logged eliminated } \\
\text { outliers but after logging the values were still skewed. Square root } \\
\text { transformation failed to eliminate skew. Categorization of variable } \\
\text { was done. }\end{array}$ \\
\hline Number of years smoked & $\mathrm{Z}=2.66, \mathrm{p}<0.008$ & $\mathrm{Z}=-2.92, \mathrm{p}<0.004$ \\
\hline $\begin{array}{l}\text { Number of years smoked } \\
\text { sqr }\end{array}$ & $\mathrm{Z}=-0.89, \mathrm{p}=0.38$ & $\begin{array}{l}\mathrm{Z}=-5.17, \mathrm{p}<0.001 \text { Since } \log \text { transformation yielded non-normality } \\
\text { and skewed, a square root transformation was done. This } \\
\text { eliminated skewness but Kurtosis still was present. It remains } \\
\text { normally distributed with } S Q R \text { transformation. }\end{array}$ \\
\hline $\begin{array}{l}\text { Number cigarette years (\# } \\
\text { cig day } \mathrm{x} \# \text { year smoke) }\end{array}$ & $z=11.30, p<0.001$ & $\begin{array}{l}\mathrm{Z}=8.61, \mathrm{p}<0.001 \text {. Transformation could not eliminate skew. } \\
\text { Variable was categorized. }\end{array}$ \\
\hline \multicolumn{3}{|l|}{ Oral findings: } \\
\hline Plaque score & $Z=3.29, p<0.001$ & $\mathrm{Z}=-6.36, \mathrm{p}<0.001$ \\
\hline Plaque score SIN & $\mathrm{Z}=2.05, \mathrm{p}<0.04$ & $\begin{array}{l}\mathrm{Z}=-26.60, \mathrm{p}<0.001 \text {. Although this variable had no outliers and was } \\
\text { normally distributed, A SIN transformation could not eliminate } \\
\text { Skewness and Kurtosis. Log and SQR transformations were } \\
\text { inappropriate and did not eliminate skew or Kurtosis. The variable } \\
\text { was categorized. }\end{array}$ \\
\hline Bleeding score & $\mathrm{Z}=6.09, \mathrm{p}<0.001$ & $\mathrm{Z}=1.93, \mathrm{p}<0.05$ \\
\hline Bleeding score SIN & $\mathrm{Z}=1.89, \mathrm{p}<0.05$ & $\begin{array}{l}\mathrm{Z}=-28.12, \mathrm{p}<0.0001, \mathrm{SIN} \text { transformation did not yield normality or } \\
\text { eliminate skew or Kurtosis. The variable was categorized. }\end{array}$ \\
\hline Medical health variables: & $\mathrm{Z}=-9.24, \mathrm{p}<0.001$ & $\begin{array}{l}Z=4.17, \mathrm{p}<0.001 \text {. This variable was not normally distributed and } \\
\text { had outliers. Logging and } S Q \text { rooting would not eliminate these } \\
\text { problems or the problems of skewness and Kurtosis, therefore it } \\
\text { was categorized. }\end{array}$ \\
\hline Systolic blood pressure & $\mathrm{Z}=4.72, \mathrm{p}<0.001$ & $\begin{array}{l}\mathrm{Z}=1.01, \mathrm{p}=0.31 \text {. This variable was not normally distributed and the } \\
\text { skewness was significant. Logging and } \mathrm{SQR} \text { made it normally } \\
\text { distributed but did not eliminate skewness therefore the variable } \\
\text { was categorized }<140 / \geq 140\end{array}$ \\
\hline Diastolic blood pressure & $\mathrm{Z}=3.94, \mathrm{p}<0.0001$ & $\begin{array}{l}\mathrm{Z}=4.06, \mathrm{p}<0.001 \text {. This variable was not normally distributed and } \\
\text { skewness and Kurtosis significant. Logging and } \mathrm{SQ} \text { root did not } \\
\text { yield normality and skewness/Kurtosis remained a concern, } \\
\text { therefore categorized category }<90 / \geq 90 \text {. }\end{array}$ \\
\hline
\end{tabular}


test under a subfunction of NCSS and a critical value of .05. In a normal distribution, the values of skewness and kurtosis must be zero.

Since Age was normally distributed and not skewed, the observed kurtosis was not believed to be a concern. The number of Cigarettes/Day was categorized since the variable remained skewed after transformations were attempted. The Number of Years Smoked was squared to eliminate skewness. A sine transformation of Plaque Score and Bleeding of Probing could not eliminate skewness or kurtosis, so they were categorized. The Number of Teeth and Systolic/Diastolic Blood Pressure were categorized due to inability to normalize their distribution and eliminate skewness/kurtosis. Results are also outlined in Table 8.8.

The only variable which remained continuous subsequent to initial data screening and univariate analysis was Age. The average age of participants $(\mathrm{n}=382)$ at the time of their periodontal examination was 55.2 years, $\mathrm{SD}=12.7$ with ages ranging from $35-87$ years.

\subsubsection{Overview of Discrete Variables}

Descriptive analysis and crosstabs subfunctions of NCSS were used to determine the distribution of potential explanatory variables. The results are outlined in Table 8.9. 
TABLE 8.9 Description of categorical variables

\section{Telephone interview $(n=382)$}

Variable

Sociodemographic Factors:

Gender

Marital status

Employment status

Occupation

Education level

Income level

\section{Description of variables by number and percentage}

$$
\text { Female }=223(58.4 \%) \quad \text { Male }=159(41.6 \%)
$$

Single (never married) $=34(8.9 \%)$

Married/Common Law (and not separated) $=257(67.3 \%)$

Divorced/Separated $=54(14.1 \%)$

Widowed/Widower $=37(9.7 \%)$

$\mathrm{DK} / \mathrm{NR}=0$

Full-time $(\geq 35 \mathrm{hr} /$ week $)=105(27.5 \%)$

Part-time $(<35 \mathrm{hr} / \mathrm{week})=65(17.0 \%)$

Unemployed $=10(2.6 \%)$

Laid Off $=3(0.8 \%)$

Retired $=137(35.9 \%)$

Homemaker $=42(11.0 \%)$

Student $=4(1.0 \%)$

Other $=16(4.2 \%)$

$\mathrm{DK} / \mathrm{NR}=0$

Professional $=47(12.3 \%)$

Manager/Official/Proprietor $=23(6.0 \%)$

Office/Clerical/Sales $=95(24.9 \%)$

Foreman $=86(22.6 \%)$

Unskilled $=85(22.3 \%)$

Not applicable $=45(11.8 \%)$

(42 homemakers \& 3 non-employed students)

Missing Value $=1(0.26 \%)$

No Schooling $=2(0.5 \%)$

Elementary (K-9): Some $=52(13.7 \%)$; Completed $=17(4.5 \%)$

Secondary (10-12): Some $=71(18.7 \%)$;

Completed $=60(15.8 \%)$

Community/Technical College:

Some $=13(3.4 \%) ;$ Completed $=63(16.6 \%)$

University: Some $=37(9.8 \%)$; Completed $=36(9.5 \%)$

Graduate Studies/ Professional Studies (Masters, PhD, Law, MD, DMD): Some = $10(2.6 \%)$; Complete $=17(4.5 \%)$

$$
\begin{aligned}
& \text { Under } \$ 12,000=49(13.3 \%) \\
& \$ 12,000-\$ 24,999=123(33.5 \%) \\
& \$ 25,000-\$ 49,999=131(35.7 \%) \\
& \$ 50,000-\$ 74,999=23(6.3 \%) \\
& \geq \$ 75,000=16(4.4 \%) \\
& \text { Refusal to answer }=25(6.8 \%) \\
& \text { DK/NR }=15
\end{aligned}
$$




\section{Behavior:}

Toothbrushing frequency

$$
\begin{aligned}
& \geq 1 \mathrm{x} / \text { day }=345(90.3 \%) \\
& \geq 1 \mathrm{x} / \text { week }=34(8.9 \%) \\
& \geq 1 \mathrm{x} / \text { month }=2(0.5 \%) \\
& \text { rarely }=0 \\
& \text { never }=1(0.3 \%) \\
& \text { DK/NR }=0
\end{aligned}
$$

Flossing frequency

$$
\begin{aligned}
& \geq 1 \mathrm{x} / \text { day }=93(24.4 \%) \\
& \geq 1 \mathrm{x} / \text { week }=127(33.3 \%) \\
& \geq 1 \mathrm{x} / \text { month }=42(11.0 \%) \\
& \text { rarely }=75(19.7 \%) \\
& \text { never }=44(11.5 \%) \\
& \mathrm{DK} / \mathrm{NR}=1(0.26 \%)
\end{aligned}
$$

Cigarette smoking status

Never smoked $=191(50 \%)$

Ever smoked $=191(50 \%)$

\section{Chronic conditions/ diseases:}

Diagnosed diabetes

Diagnosed hypertension

(high blood pressure)

Diagnosed MI

Diagnosed angina

Diagnosed artero/artherosclerosis

\section{Use of specific medications:}

Dilantin

Cyclosporin

Nifedipine

Oral contraceptives / estrogens/

Progesterone / provera: ( $\mathrm{n}=223$ females)

Other medicines regularly

Anti-inflammatory $>3 \mathrm{x} /$ week ( $n=152$ who report taking other regular medicines $>3 \mathrm{x}$ week)

HBP meds

( $\mathrm{n}=96$ who reported being told

they had HBP)

Insulin

(self reported diabetes $=22$,

Taking diabetic medications $=17$ )

Oral meds diabetes

(self reported diabetes $=22$,

Taking diabetic medications $=17$ )

$$
\begin{array}{ll}
\begin{array}{l}
\text { No }=360(94.2 \%) \\
\text { Borderline }=6(1.6 \%)
\end{array} & \text { Yes }=16(4.2 \%) \\
\text { No }=286(74.9 \%) & \text { Yes }=96(25.1 \%) \\
\text { No }=370(96.9 \%) & \text { Yes }=12(3.1 \%) \\
\text { No }=355(92.9 \%) & \text { Yes }=27(7.1 \%) \\
\text { No }=361(94.5 \%) & \text { Yes }=21(5.5 \%)
\end{array}
$$

$$
\begin{array}{ll}
\text { No }=380(99.5 \%) & \text { Yes }=2(0.5 \%) \\
\text { No }=382(100 \%) & \text { Yes }=0 \\
\text { No }=379(99.2 \%) & \text { Yes }=3(0.8 \%) \\
\text { No }=80(35.9 \%) & \text { Yes }=143(64.1 \%) \\
\text { No }=231(60.5 \%) & \text { Yes }=151(39.5 \%) \\
\text { No }=80(52.6 \%) & \text { Yes }=72(47.4 \%)
\end{array}
$$

$$
\text { No }=60(62 . \%) \quad \text { Yes }=36(37.5 \%)
$$$$
\mathrm{NA}=286
$$$$
\text { No }=12(70.6 \%) \quad \text { Yes }=5(29.4 \%)
$$$$
\mathrm{NA}=360
$$

$$
\text { No }=9(52.9 \%) \quad \text { Yes }=8(47.1 \%)
$$


Other

Level of life stress

No stress $=26(6.8 \%)$

Slight Stress $=108(28.4 \%)$

Moderate Stress $=181(47.6 \%)$

Extreme Stress $=65(17.1 \%)$

$\mathrm{DK} / \mathrm{NK}=2(0.52 \%)$

\section{Dental/medical chart information $(n=382)$}

\section{Oral findings:}

$\begin{array}{lll}\begin{array}{l}\text { Regular dental visiting } \\ \text { (Reported At H\&E) }\end{array} & \text { No } & 247(66.2 \%) \\ \text { Missing Value } & \text { Yes } & 126(33.8 \%) \\ \text { Bleeding on probing } & \bullet(8) & \\ \text { (Bop) } & \text { Slight }(0-33 \%) & 205(57.6 \%) \\ & \text { Moderate }(34-66 \%) & 112(34.3 \%) \\ & \text { Severe }(\geq 67 \%) & 29(8.1 \%) \\ \text { Plaque } & \text { Missing }(26) & \\ & \text { Slight }(0-33 \%) & 106(30.3 \%) \\ & \text { Moderate }(34-66 \%) & 143(40.9 \%) \\ \text { \# teeth present } & \text { Severe }(\geq 67 \%) & 101(28.8 \%) \\ & \text { Missing }(32) & \\ \text { \# Teeth Present } & \geq 10 & 354(92.7 \%) \\ & <10 & 28(7.3 \%) \\ \text { \# Teeth Present } & \geq 20 & 299(78.3 \%) \\ & <20 & 83(21.7) \\ & \geq 25 & 185(48.4) \\ & <25 & 197(51.6)\end{array}$

General health variables:

$\begin{array}{lll}\text { SBP }>\text { 140 } & \text { No } & 288(80.4) \\ & \text { Yes } & 70(19.6) \\ \text { MBP }>\text { 90 } & \text { Missing Value (24) } & \\ & \text { No } & 318(88.8) \\ & \text { Yes } & 40(11.2)\end{array}$

\section{Sociodemographic Factors:}

Of those participating in the study $(\mathrm{n}=382), 58.4 \%$ were female and $41.6 \%$ male.

The majority of participants were married or living common law (67.3\%) while $8.9 \%$ were single, $14.1 \%$ divorced or separated and $9.7 \%$ widowed. This variable was subsequently collapsed into: partner (67.3\%) and single (32.7\%). Approximately $36 \%$ of the participants were retired, and 
$11 \%$ homemakers, while $27.5 \%$ reported to be employed full-time and $17 \%$ part-time. Forty-five percent of respondents reported semi-skilled occupations, an additional $24.9 \%$ were clerical/sales and $18.6 \%$ held professional/ managerial positions. There was only one missing value for occupation. Over half $(53.2 \%)$ reported graduation from secondary (Grade 12) school as their highest level of education while $9.5 \%$ graduated from university and another $4.5 \%$ completed professional/graduate studies. There were 12 missing values for education but there appeared to be no difference between cases and controls regarding the frequency of missing education data when categorized, $\leq \mathrm{Gr} 12$ vs $>$ Gr 12 . Nearly half (46.8\%) reported an annual gross family household income of $<\$ 25,000$ while most (82.5\%) reported their income as $<\$ 50,000$. Only $4.19 \%$ reported income $\geq$ $\$ 75,000 /$ year and $13.3 \%$ under $\$ 12,000 /$ year. Eleven percent of the respondents either did not know their family income or refused to divulge the information. No difference between the cases and controls was observed in the frequency of missing income data when categorized, $<\$ 25,000 \mathrm{vs} \geq$ $\$ 25,000$.

\section{Behaviors:}

The vast majority (90.3\%) of individuals reported to brush their teeth once or more/day while only $24.4 \%$ reported the same frequency of flossing. Equal numbers of individuals reported to have been regular smokers at some time (50\%) as not (50\%). While $13.4 \%$ had > 500 Cigarette-Index (a continuous measure of life-time exposure to smoking), $26.7 \%$ had $1-250$ cigarettes index (\# cig/day $\mathrm{x}$ \# yrs smoked). To calculate the actual number of cigarettes smoked in one's lifetime, the cigarette years' index could be multiplied by 365 . Regular dental visiting was reported by $33.8 \%$ of the individuals. 


\section{Chronic Conditions/Diseases:}

The prevalence of 'Diagnosed Diabetes' was $4.2 \%$ with an additional $1.6 \%$ reporting, "borderline diabetes" which could represent an overall prevalence of diagnosed diabetes of 5.8\%. Approximately 25\% reported having been told by a doctor or nurse that they had hypertension/high blood pressure while only $3.1 \%$ had been diagnosed with a Myocardial Infarction, 7.1\% with Angina Pectoris, and 5.5\% with Arterio/Atherosclerosis.

\section{Use of Study Medications:}

Use of specific study medications was relatively rare but reported for Dilantin/Phenytoin $(\mathrm{n}=2,0.5 \%)$, Nifedipine/Adalat $(\mathrm{n}=3,0.8 \%)$ and Cyclosporin $\mathrm{n}=0$. Due to inadequate levels of exposure, even when combined, these variables were not analyzed further.

Prior exposure to hormonal replacement therapy/oral contraceptives (Estrogen/Progesterone) was relatively common (64.1\%) among women. Of those subjects who reported taking "Any Medication" more than $3 \mathrm{x} /$ week, $47.4 \%$ took anti-inflammatory medications. Of the 96 individuals who had been told that they had Hypertension, 37.5\% had taken medication for the condition. Insulin was used by six (33.3\%) of the 17 individuals under treatment for diabetes.

\section{Oral Findings:}

When BOP was categorized, slight bleeding on probing $(0-33 \%$ of surfaces) was found among $57.6 \%$ of the subjects while moderate (34-66\%) was found among $34.3 \%$ and severe bleeding ( $\geq 67 \%$ ) among $8.1 \%$. Due to inadequate cell size associated with $>67 \%$ BOP, a decision was made to use $50 \%$ BOP in subsequent analyses. There were 26 subjects for whom BOP 
scores were unavailable. As described earlier, missing BOP scores were randomly distributed among all age groups, both genders and levels of education. However, there were more missing BOP scores for controls ( $\mathrm{n}=$ 24) than cases ( $n=2$ ) which may be explained by failure of clinicians to calculate a BOP score when there no clinical evidence of inflammation. There was a tendency for more missing BOP scores when there were more teeth present, that is, 18 were missing among those who had most $(\geq 20)$ of their teeth while only four were missing when the subjects had fewer than 10 teeth present and another four were missing among those with $10-19$ present. This situation suggests that BOP scores were taken less often on periodontally healthy individuals. There were more missing BOP scores among those who brushed $\geq 1$ /day $(n=22)$ than those who brushed $<1 /$ day $(n=4)$. The impact of missing Bleeding on Probing scores on the results of the current study could be an under-estimate of the association between BOP and periodontitis, assuming that the missing controls had low BOP.

The prevalence of categorized PS was: slight $(0-33 \%$ of surfaces $)=$ $30.3 \%$; moderate $(34-66 \%$ of surfaces $)=40.9 \%$; severe $(\geq 67 \%$ of surfaces $)=$ 28.9\%). There were 32 subjects for whom Plaque Scores were missing.

As mentioned earlier, missing Plaque Scores were randomly distributed among all age groups, both genders and levels of education. However, there were more missing plaque scores among cases $(n=22)$ than controls $(n=10)$ and there was a trend for more missing PSs when subjects had more teeth present i.e., there were 20 missing scores among those with $\geq 20$ teeth present and only four missing among those who had fewer than 10 teeth present. There were more scores missing among those who brushed $\geq 1 \mathrm{x}$ /day $(\mathrm{n}=14)$ than those who didn't $(\mathrm{n}=2)$. The reason for missing PS may be that clinicians had a tendency not to calculate it among patients in whom the oral hygiene appeared excellent or in advanced cases 
where plaque was generalized. The impact of missing Plaque Scores in the current study is that the effect of plaque on AP could be somewhat underestimated assuming that the missing control subjects had low plaque scores. There were $51.6 \%$ of participants who had fewer than 25 teeth present, while $21.7 \%$ had fewer than 20 teeth and $7.3 \%$ had fewer than 10 teeth present.

\section{Other:}

Extreme stress was reported by $17.1 \%$ of the respondents while $6.8 \%$ reported no stress. A number of categorical variables were further collapsed due to insufficient cell size. The results are reported in Table 8.10.

TABLE 8.10 Collapsed categorical variables $(\mathrm{n}=382)$

\begin{tabular}{|c|c|c|c|}
\hline & NEW CATEGORIES & $\#$ & $\%$ \\
\hline \multicolumn{4}{|l|}{$\begin{array}{l}\text { Sociodemographic } \\
\text { Factors: }\end{array}$} \\
\hline Age: & $\begin{array}{l}35-54 \text { year } \\
55-64 \text { year }\end{array}$ & $\begin{array}{r}185 \\
92\end{array}$ & $\begin{array}{l}(48.4) \\
(24.1)\end{array}$ \\
\hline \multirow{3}{*}{ Marital status: } & $\geq 65$ year & 105 & (27.5) \\
\hline & Partner & 257 & 67.3 \\
\hline & Single & 125 & 32.7 \\
\hline \multirow{4}{*}{ Employment: } & Employed (FT/PT) & 170 & 44.5 \\
\hline & Unemployed/Laid Off & 13 & 3.4 \\
\hline & Retired & 137 & 35.9 \\
\hline & Homemaker & 42 & 11.0 \\
\hline \multirow{3}{*}{ Employment } & $\begin{array}{l}\text { Other/Student } \\
\mathrm{FT} / \mathrm{PT}\end{array}$ & 20 & 5.2 \\
\hline & Laid Off/Unemployed & $\begin{array}{r}170 \\
13\end{array}$ & $\begin{array}{l}44.5 \\
3.4\end{array}$ \\
\hline & Retired/Homemaker/ Student & 199 & $\begin{array}{l}0.4 \\
52.1\end{array}$ \\
\hline \multirow{2}{*}{ Employment } & $\mathrm{FT/PT}$ & 170 & 44.5 \\
\hline & All other & 212 & 55.5 \\
\hline \multirow{3}{*}{ Occupation } & Professional/Managerial & 71 & 18.6 \\
\hline & $\begin{array}{l}\text { Clerical/Sales } \\
\text { Semi-Skilled/4nckillar }\end{array}$ & 95 & 24.9 \\
\hline & $\begin{array}{l}\text { Semi-Skilled/Unskilled } \\
\text { Other }\end{array}$ & $\begin{array}{r}171 \\
45\end{array}$ & $\begin{array}{l}44.8 \\
118\end{array}$ \\
\hline \multirow{2}{*}{ Occupation } & White Collar & $\begin{array}{r}45 \\
166\end{array}$ & 11.8 \\
\hline & Blue Collar / Housewife / Retired / Student & 166 & 43.5 \\
\hline \multirow[t]{4}{*}{ Highest level education } & $\begin{array}{l}\text { No formal education/some or graduated elementary } \\
\text { (Grade 9) }\end{array}$ & $\begin{array}{r}216 \\
74\end{array}$ & $\begin{array}{l}56.5 \\
19.4\end{array}$ \\
\hline & Some/Graduate Secondary (Grade 12) & 131 & 34.3 \\
\hline & Some/Grad Technical & 76 & 19.9 \\
\hline & $\begin{array}{l}\text { Some/Grad University/Graduate/ Professional Degree } \\
\text { Other (DK/NB) }\end{array}$ & 100 & 26.2 \\
\hline Education & $\begin{array}{l}\text { Other (DK/NR) } \\
>\text { Grade } 12 \\
\leq \text { Grade } 12\end{array}$ & $\begin{array}{r}1 \\
177 \\
205\end{array}$ & $\begin{array}{l}0.3 \\
46.3 \\
53.7\end{array}$ \\
\hline Income & $\begin{array}{l}<\$ 12,000 \\
\$ 12-24,999\end{array}$ & $\begin{array}{r}49 \\
123\end{array}$ & $\begin{array}{l}13.3 \\
33.5\end{array}$ \\
\hline
\end{tabular}




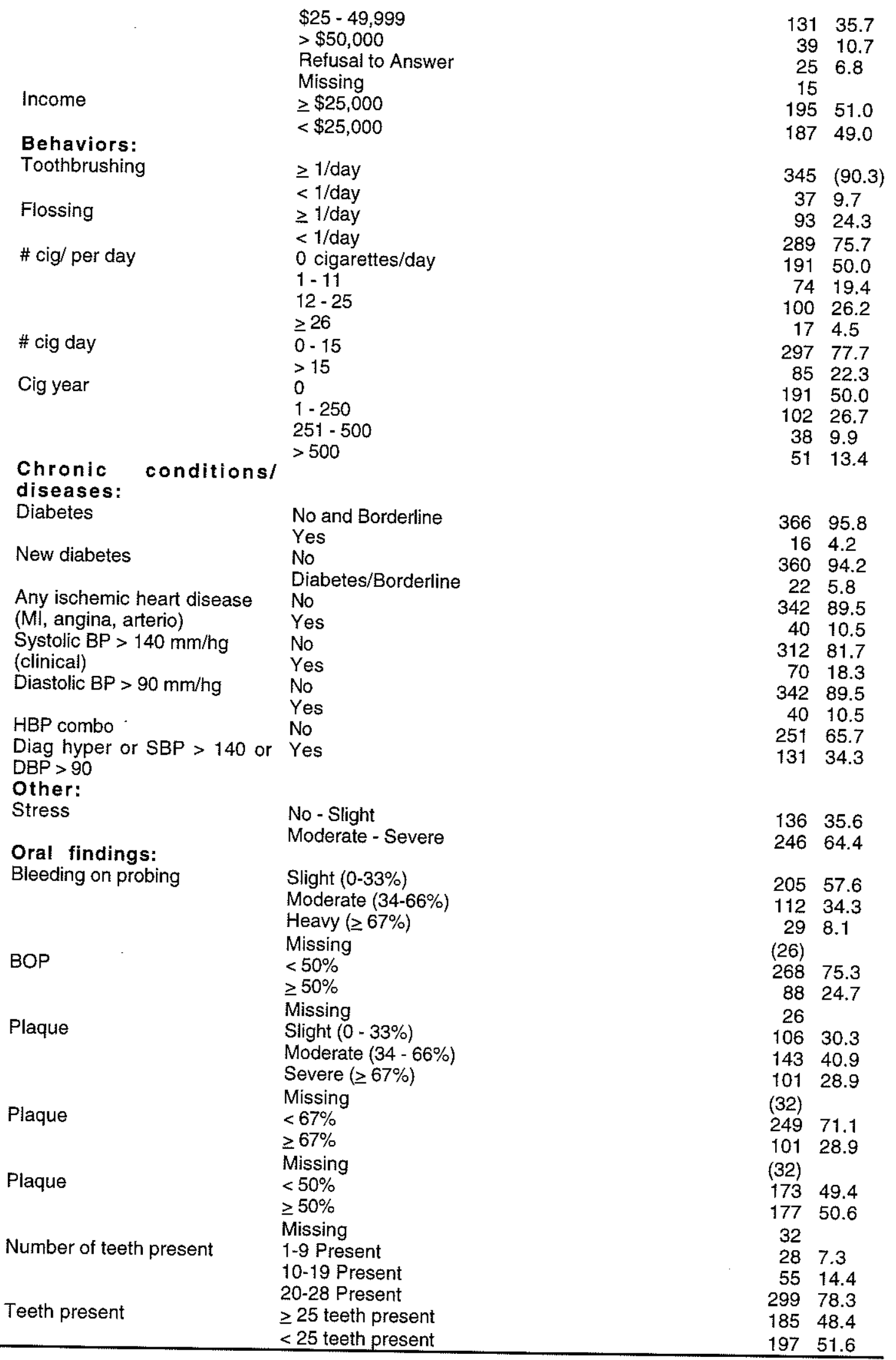




\subsubsection{Comparative Exposure To Variables Of Interest}

\section{Cases vs Controls:}

The distribution of key explanatory variables among controls and cases was analyzed and compared using Chi-square or t-test statistics. Analyses were carried out using the six Diagnostic Criteria (A-F) which were discussed in Sections 6.4 - 6.6. The results were reported in Table 6.4.

\section{Exclusive Use Of Criterion F As Outcome:}

Since Diagnostic Criterion F $(\geq 1$ site with $>3 \mathrm{~mm}$ distance radiographically between $\mathrm{AC}$ and $\mathrm{CEJ}$ or $\geq 1$ site with $\geq 7 \mathrm{~mm} \mathrm{PD}$ ) was previously identified as the optimum method of defining "case", all subsequent analyses were carried out using it as the outcome $(\mathrm{n}=362)$. Subfunctions for NCSS statistical software program were used in the following analyses.

The t-test and Chi-square tests were used to investigate differences between cases and controls regarding continuous and categorical variables respectively. The level of significance was set at 5\% level (Fleiss, 1981; Kleinbaum et al 1982). Results are reported in Table 8.11.

The unadjusted odds ratios (OR) for explanatory variables were calculated using Logistic Regression whereby the resulting beta estimates were exponentiated (anti-logged) to determine the unadjusted odds ratio. The unadjusted OR was used to measure the degree or magnitude of association between the explanatory and dependent variables. The likelihood ratio Chi-square was used to assess the significance of odds ratios. The level of significance was set at 5\% (Fleiss 1981; Kleinbaum et al 1982). The confidence limits of each OR was calculated by the formula:

\section{C.I. $=\exp$ [ Beta estimate $+/-2 \times$ Standard Error of ßeta estimate]}


The results for these analyses are reported in Table 8.11.

\section{Sociodemographic Factors:}

Among demographic variables, both age and gender showed a significant difference between cases and controls. The mean age of controls was 49.8 years, $\mathrm{SE}=0.93$ while that of cases was $58.0, \mathrm{SE}=0.85, \mathrm{t}=-6.5, \mathrm{p}<$ 0.0001 . When age was categorized as: $35-54$ and $\geq 55$ years, there was also a significant difference between cases and control with a higher proportion of the younger subjects $(70.1 \%)$ being controls, $\mathrm{x}^{2}=40.0,1 \mathrm{df}, \mathrm{p}<0.0001, \mathrm{OR}=$ $4.08, \mathrm{CI}=2.59-6.43$. Being $\geq 55$ years old is associated with a 4.08 times increased risk of a history of periodontitis compared to those aged $35-54$ years.

There was a higher proportion of females (64.9\%) among controls than cases, $\mathrm{x}^{2}=4.9,1 \mathrm{df}, \mathrm{p}<0.03, \mathrm{OR}=1.62, \mathrm{CI}=1.04-2.51$. Being male was associated with 1.62 times increased risk of a history of periodontitis compared to females.

Among socioeconomic variables, several showed a significant difference between cases and controls including: a) marital status: i.e., more of those with partners $(74.0 \%)$ were controls than cases, $x^{2}=5.4,1 \mathrm{df}, \mathrm{p}$ $<0.02, \mathrm{OR}=1.71, \mathrm{CI}=1.07-2.72$. Being single as opposed to having a partner increased risk by 1.71 times; b) education, i.e., a higher proportion of cases had grade 12 or less education $(52.2 \%)$ than controls, $\mathrm{x}^{2}=5.7,1 \mathrm{df}, \mathrm{p}$ $<0.02, \mathrm{OR}=1.67, \mathrm{CI}=1.09-2.56$. Having a grade 12 education or less increased risk by 1.67 times compared to those with more than grade 12 education; c) employment, i.e., controls were more likely to be employed full/part time $(57.1 \%)$ versus all others, including retired/homemakers/ students/ laid off/unemployed than cases, $\mathrm{x}^{2}=13.07,1 \mathrm{df}, \mathrm{p}<0.0003, \mathrm{OR}=$ $2.17, \mathrm{CI}=1.41-3.26$. There was an increased risk of 2.17 times for those 
TABLE 8.11 Comparison of cases and controls: Crude disease-exposure association for individual key explanatory variables $(n=362)$

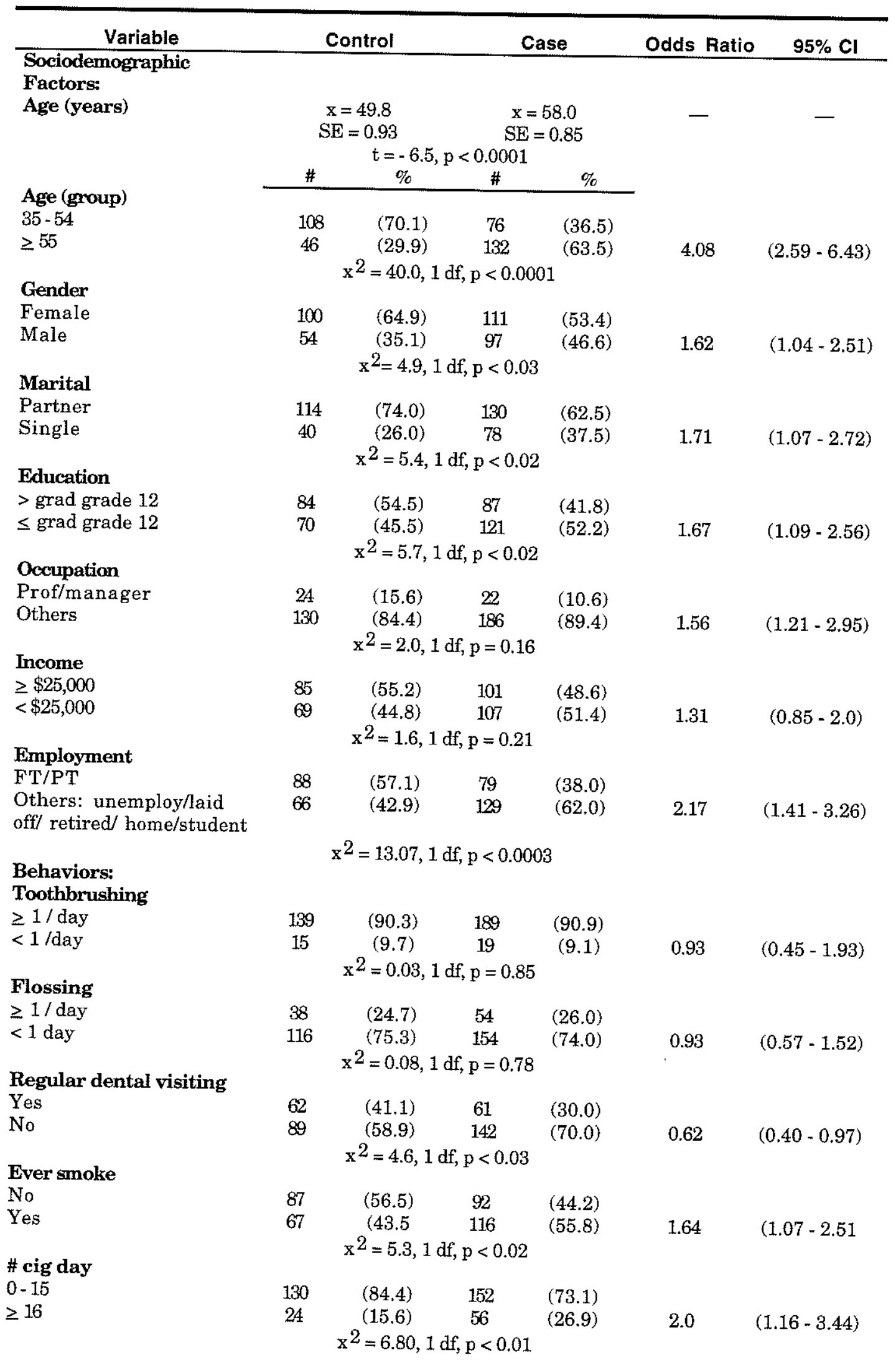




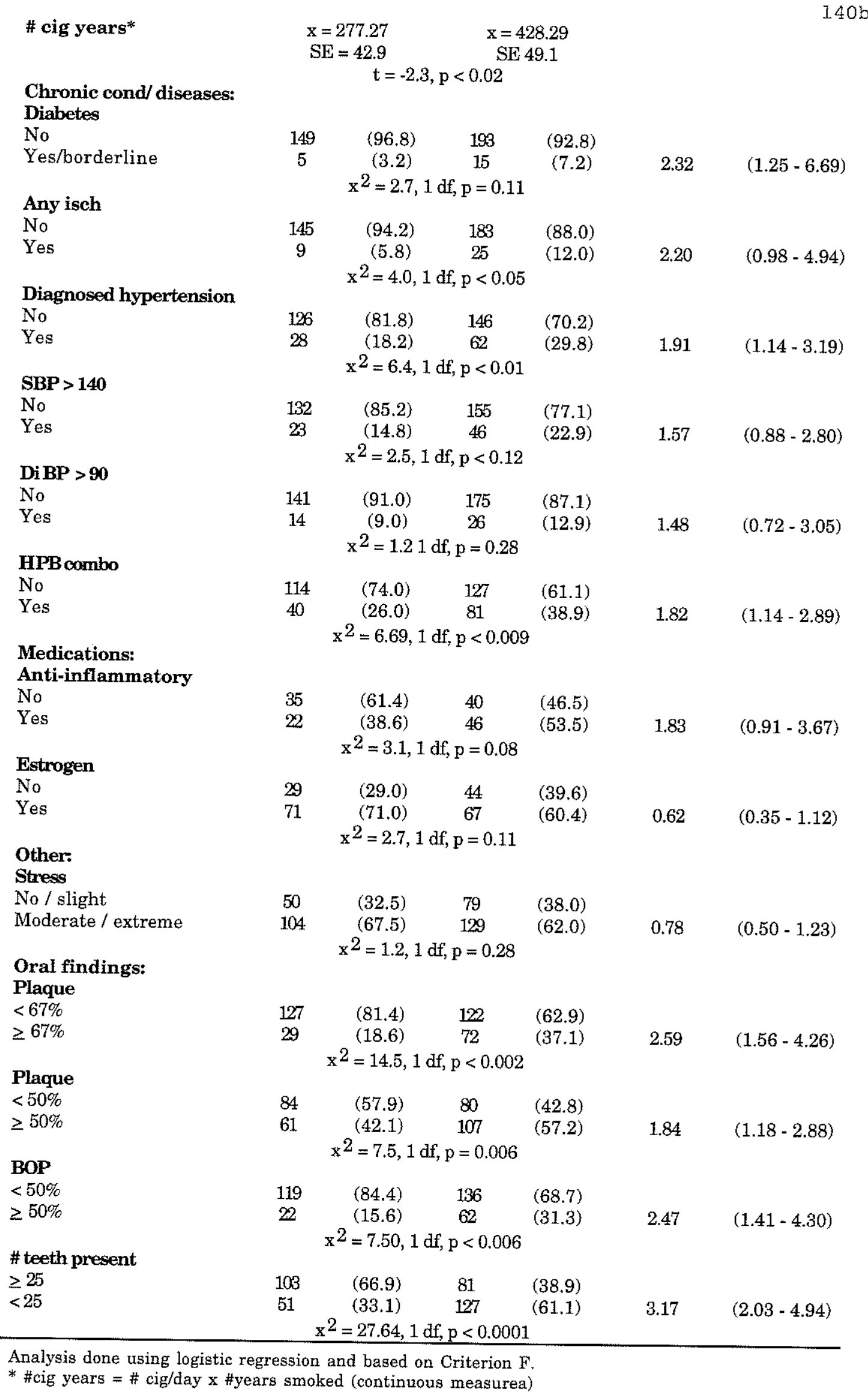


who were not employed full/part time compared to all others. There was no significant difference in income between case and controls, $\mathrm{x}^{2}=1.6,1 \mathrm{df}, \mathrm{p}=$ 0.21 .

\section{Behaviors:}

When health compromising or promoting behaviors were considered, infrequent dental visiting was found significantly more among cases $(70.0 \%)$ than among controls $\mathrm{x}^{2}=4.6,1 \mathrm{df}, \mathrm{p}<0.03, \mathrm{OR}=0.62, \mathrm{CI}=0.40$ - 0.97. There was a 0.62 times reduced risk among those who reported to have regular dental visits compared to those who did not. A significantly greater proportion of controls reported never to have smoked (56.5\%) than cases $\mathrm{x}^{2}=5.3,1 \mathrm{df}, \mathrm{p}<0.02, \mathrm{OR}=1.64, \mathrm{CI}=1.07-2.51$. There was an increased risk of 1.64 times for those who reported ever to have smoked compared to never-smokers. There was also a significant difference between cases and controls in the mean number of cigarette years, (\#cigarettes/day $\mathrm{x}$ \# years smoked) with controls mean $=277.27, \mathrm{SE}=42.9$ and cases $=428.29, \mathrm{SE}=49.1, \mathrm{p}<0.02$. A categorical cigarette index was introduced to reflect total life-exposure to smoking. (Further multiplying the cigarette index by 365 would indicate the total \#cigarette exposed to in one's lifetime.) There was no significant difference between cases and controls regarding reported frequency of toothbrushing, $x^{2}=0.03,1 \mathrm{df}, \mathrm{p}=$ 0.85 , or flossing, $x^{2}=0.08,1 \mathrm{df}, \mathrm{p}=0.78$.

\section{Chronic Conditions and Diseases:}

A significant difference between cases and controls was observed when chronic diseases/conditions were considered. There was a greater proportion of individuals who reported a history of diagnosed hypertension among cases $(29.88 \%)$ than controls $(18.2 \%), \mathrm{x}^{2}=6.4,1 \mathrm{df}, \mathrm{p}<0.01, \mathrm{OR}=$ 
$1.91, \mathrm{CI}=1.14-3.19$. Those with a history of diagnosed hypertension had an increased risk of 1.91 times compared to those without the condition. A similar pattern was found for a reported history of any ischemic heart disease (including MI, Angina Pectoris, or Arterio/Atherosclerosis), cases $(12.0 \%), \mathrm{x}^{2}=4.0,1 \mathrm{df}, \mathrm{p}<0.05, \mathrm{OR}=2.20, \mathrm{CI}=0.98-4.94$. There was a trend for more cases to have a history of diagnosed diabetes $(7.2 \%), \mathrm{x}^{2}=6.41 \mathrm{df}, \mathrm{p}$ $=0.11, \mathrm{OR}=2.32, \mathrm{CI}=1.25-6.69$, and systolic blood pressure $>140 \mathrm{~mm} / \mathrm{Hg}$ $(22.9 \%), \mathrm{x}^{2}=2.5,1 \mathrm{df}, \mathrm{p}=0.12, \mathrm{OR}-1.57, \mathrm{CI}=0.88-2.80$. No difference was observed regarding diastolic blood pressure $>90 \mathrm{~mm} / \mathrm{Hg}, \mathrm{x}^{2}=1.3,1 \mathrm{df}, \mathrm{p}=$ 0.27. A significantly greater proportion of controls failed to report a history of HBP or to have SBP > 140 or DBP $>90 \mathrm{~mm} / \mathrm{Hg}$ (HBP Combo), $\mathrm{x}^{2}=6.69,1$ df, $\mathrm{p}<0.01, \mathrm{OR}=1.82, \mathrm{CI}=1.14-2.89$. This latter "Combo" variable was introduced to increase the number of individuals who had any past evidence of hypertension $(n=121)$, either through diagnosis by physician ( $n$ $=90)$ or through clinical records of elevated SBP $>140 \mathrm{~mm} / \mathrm{Hg}(\mathrm{n}=69)$ or $\mathrm{DBP}>90 \mathrm{~mm} / \mathrm{Hg}(\mathrm{n}=40)$ from existing secondary data. Although difference between cases and controls was more significant using "Combo" than "diagnosed hypertension", it produced a lower OR.

\section{Medications:}

Regarding the medications estrogen/progesterone and antiinflammatory agents, no significant differences were found between cases and controls. There was also no difference observed in terms of exposure to life-stress $\mathrm{x}^{2}=1.2,1 \mathrm{df}, \mathrm{p}=0.28$.

\section{Dental/ Oral Findings:}

When dental observations were considered, the difference between cases and controls was significant for: a) PS when categorized as: $0-66 \%$ 
versus $\geq 67 \%, \mathrm{x}^{2}=14.5,1 \mathrm{df}, \mathrm{p}<0.0002, \mathrm{OR}=2.59, \mathrm{CI}=1.56-4.26$; or as $<0$ $50 \%$ versus $\geq 50 \%, \mathrm{x}^{2}=7.50,1 \mathrm{df}, \mathrm{p}<0.006, \mathrm{OR}=1.84, \mathrm{CI}=1.18-2.88$, controls consistently had lower plaque scores and less risk for a history of periodontitis than cases; b) BOP when categorized as: $0-50 \%$ versus $\geq 50 \%$ $\mathrm{x}^{2}=7.50,1 \mathrm{df}, \mathrm{p}<0.006, \mathrm{OR}=1.84, \mathrm{CI}=1.41-4.30$ whereby cases had higher BOP and increased risk than controls; c) 'number of teeth present' when categorized as $<25$ versus $\geq 25$ teeth present showed a significantly higher proportion of controls (66.9\%) with $\geq 25$ teeth present, $\mathrm{x}^{2}=27.64,1 \mathrm{df}, \mathrm{p}<$ $0.0001, \mathrm{OR}=3.17, \mathrm{CI}=2.03-4.94$. There was a significant increase in risk for those with $<25$ teeth present compared to those with $\geq 25$ teeth present. Presence of 25 teeth was selected for further analyses rather than 10 or 20 teeth as the latter two showed no significant difference between cases and controls.

The apparent risk of the aforementioned explanatory variables was estimated by their unadjusted odds ratios. The unadjusted OR is able to correctly indicate whether an exposure increases or reduces the risk of disease, but the magnitude of the effect may be overestimated or underestimated when key confounding variables are not controlled (Schlesselman 1982). Adjustment is discussed in section 8.7.5.

\subsubsection{Test For Dose-Response}

Logistic regression analysis was used to determine whether any of the significant explanatory variables had a dose-response effect on a history of periodontitis. Logistic regression is considered a basic model for doseresponse relationships (Cornfield et al, 1961). 
The Dose-Response Effect of the following variables was tested:
1. Age Category:
2. \# Cigarettes/Day:
3. \# Years Smoked:
4. Cigarette-Index:
5. Cigarette-Index:
6. Cigarette-Index:
7. Plaque Category:
$35-54 ; 55-64 ; \geq 65$ Years
$0 ; 1-11 ; 12-25 ; \geq 26$
$0 ; 1-5 ; 6-15 ; 16-25 ; \geq 26$
$0 ; 1-250 ; 251-500 ;>500$
$0 ; 1-50 ; 51$ - 300;>300
8. Bleeding On Probing:
$0 ; 1-300 ; 301-500 ;>500$
$0-33 \% ; 34-66 \% ; \geq 67 \%$
$0-33 \% ; 34-66 \% ; \geq 67 \%$

The results of the unadjusted dose response odds ratios are reported in Table 8.12. Bleeding on Probing demonstrated a significant dose response effect with an increased $\mathrm{OR}=2.42 \mathrm{CI}=1.48-3.96$ for those with 34 - $66 \%$ BOP and $\mathrm{OR}=6.18, \mathrm{CI}=2.02-3.07$ for those with $\geq 67 \%$ BOP when compared to those with $\leq 33 \%$ BOP. There was a significant dose-response effect with age whereby those aged $55-64$ years had an $\mathrm{OR}=2.90, \mathrm{CI}=1.61$ 4.85 , and those $\geq 65$ years had an $\mathrm{OR}=6.08, \mathrm{CI}=3.33-11.11$, compared to those aged $35-54$ years. There was no dose-response effect for number of cigarettes/day but there was for number of years smoked. These two variables were combined to provide information on total life-time exposure to cigarette smoking in the variable, number of cigarette-index. There was a dose-response effect of the cigarette-index regardless of how it was categorized. A decision was made to categorize it 0;1 - 300; $301-500$; and > 500 for subsequent analyses. The dose-response effect of plaque was significant.

\subsubsection{Age- and Gender-Adjusted Odds Ratios:}

To investigate whether the crude disease-exposure association continued to exist when adjustment was made for potential confounding variables, and to determine whether the magnitude of the odds ratio was 
TABLE 8.12 Dose response to key explanatory variables (unadjusted)

\begin{tabular}{|c|c|c|c|c|c|}
\hline & & $\begin{array}{c}\text { Overall } \\
(n=362)\end{array}$ & $\begin{array}{c}\text { Control } \\
(n=154)\end{array}$ & $\begin{array}{c}\text { Case } \\
(n=208)\end{array}$ & $\begin{array}{l}\text { Odds Ratio } \\
\text { (CI 95\%) }\end{array}$ \\
\hline Age (group) & $\begin{array}{l}35-54 \\
55-64 \\
\geq 65\end{array}$ & $\begin{array}{l}184(50.8) \\
83(22.9) \\
95(26.2) \\
\text { Chi Squ }\end{array}$ & $\begin{array}{c}108(58.7) \\
28(33.7) \\
18(18.9) \\
\mathrm{x}^{2}=43.9,2 \mathrm{~d}\end{array}$ & $\begin{aligned} 76(41.3) \\
55(66.3) \\
77(81.1) \\
0.0001\end{aligned}$ & $\begin{array}{c}1.00 \\
2.90(1.61-4.8) \\
6.08(3.33-11.11)\end{array}$ \\
\hline \#Cig / day & $\begin{array}{c}0 \\
1-11 \\
12-25 \\
\geq 26\end{array}$ & $\begin{array}{l}179(49.4) \\
71(19.6) \\
96(26.5) \\
16(4.4) \\
\text { Chi Sq }\end{array}$ & $\begin{array}{c}87(48.6) \\
27(38.0) \\
35(36.5) \\
5(31.3) \\
x^{2}=5.57,3\end{array}$ & $\begin{array}{r}92(51.4) \\
44(62.0) \\
61(63.5) \\
11(68.8) \\
<0.14\end{array}$ & $\begin{array}{c}1.00 \\
1.54(0.87-2.73) \\
1.65(0.98-2.77) \\
2.08(0.68-6.37)\end{array}$ \\
\hline \#Year/Smoked & $\begin{array}{c}0 \\
1-5 \\
6-15 \\
16-25 \\
\geq 26\end{array}$ & $\begin{array}{l}179(49.4) \\
38(10.5) \\
44(12.2) \\
48(13.3) \\
53(14.6) \\
\text { Chi Squ }\end{array}$ & $\begin{array}{c}87(48.6) \\
18(47.4) \\
23(52.3) \\
17(35.4) \\
9(17.0) \\
x^{2}=19.9,4 \mathrm{~d}\end{array}$ & $\begin{array}{c}92(51.4) \\
20(52.6) \\
21(47.7) \\
31(64.6) \\
44(83.0) \\
<0.0005\end{array}$ & $\begin{array}{c}1.00 \\
1.05(0.51-2.15) \\
0.86(0.44-1.69) \\
1.72(0.88-3.38) \\
4.62(2.10-10.19)\end{array}$ \\
\hline Cig index & $\begin{array}{c}0 \\
1-250 \\
251-500 \\
>500\end{array}$ & $\begin{array}{l}179(49.4) \\
98(27.1) \\
36(9.9) \\
49(13.5) \\
\text { Chi Squa }\end{array}$ & $\begin{array}{c}87(48.6) \\
46(46.9) \\
12(33.3) \\
9(18.4) \\
=16.43,3 d\end{array}$ & $\begin{array}{l}92(51.4) \\
52(53.1) \\
24(66.7) \\
40(81.6) \\
<0.0009\end{array}$ & $\begin{array}{c}1.00 \\
1.07(0.65-1.77) \\
1.89(0.88-4.08) \\
4.20(1.90-9.32)\end{array}$ \\
\hline Cig index* & $\begin{array}{c}0 \\
1-50 \\
51-300 \\
>300\end{array}$ & $\begin{array}{l}179(49.4) \\
44(12.2) \\
64(17.7) \\
75(20.7) \\
\text { Chi Squa }\end{array}$ & $\begin{array}{l}87(48.6) \\
21(47.7) \\
28(43.8) \\
18(24.0) \\
2=16.43,30\end{array}$ & $\begin{array}{l}92(51.4) \\
23(52.3) \\
36(56.2) \\
57(76.0) \\
<0.0009\end{array}$ & $\begin{array}{c}1.00 \\
1.04(0.51-2.03) \\
1.23(0.68-2.19) \\
3.00(1.61-5.56)\end{array}$ \\
\hline Cig index* & $\begin{array}{c}0 \\
1-300 \\
301-500 \\
>500\end{array}$ & $\begin{array}{l}179(49.5) \\
108(29.8) \\
26(7.2) \\
49(13.5) \\
\text { Chi Sque }\end{array}$ & $\begin{array}{c}87(48.6) \\
49(45.4) \\
9(34.6) \\
9(18.4) \\
x^{2}=10.64,2\end{array}$ & $\begin{array}{r}92(51.4) \\
59(54.6) \\
17(65.4) \\
40(81.6) \\
<0.005\end{array}$ & $\begin{array}{c}1.00 \\
1.10(0.68-1.78) \\
1.86(0.85-4.06) \\
4.22(1.19-9.35)\end{array}$ \\
\hline Plaque & $\begin{array}{c}0-33 \% \\
34-66 \% \\
\geq 67 \% \\
30 \text { missing }\end{array}$ & $\begin{array}{c}99(29.8) \\
139(41.9) \\
94(28.3) \\
\text { Chi Squ }\end{array}$ & $\begin{array}{c}51(51.5) \\
65(45.8) \\
29(30.9) \\
\mathrm{x}^{2}=9.30,2\end{array}$ & $\begin{array}{l}48(48.5) \\
74(53.2) \\
65(69.1) \\
<0.01\end{array}$ & $\begin{array}{c}1.00 \\
1.20(0.71-2.05) \\
2.38(1.31-4.34)\end{array}$ \\
\hline BOP & $\begin{array}{c}0-33 \% \\
34-66 \% \\
\geq 67 \% \\
23 \text { missing }\end{array}$ & $\begin{array}{c}195(57.5) \\
117(34.5) \\
27(8.0) \\
\text { Chi Squa }\end{array}$ & $\begin{array}{c}101(51.8) \\
36(30.8) \\
4^{* *}(14.8) \\
\mathrm{x}^{2}=22.0,2 \mathrm{df}\end{array}$ & $\begin{array}{l}94(48.2) \\
81(69.2) \\
23(85.2) \\
<0.0001\end{array}$ & $\begin{array}{c}1.00 \\
2.42(1.48-3.96) \\
6.18(2.02-3.07)\end{array}$ \\
\hline
\end{tabular}


increased or diminished by the adjustment, Logistic Regression analysis was carried out for each potential explanatory variable.

Since both age and gender have previously, and in the current study, been correlated with a history of periodontitis (Miller et al 1987; van der Velden 1991; Machtei et al 1992; Locker and Leake 1993), cases and controls were analyzed using logistic regression where, in addition to entering the explanatory variable of interest, both age and gender were also entered into the model. This was done to control for effects of age and gender on the dependent variable. The effects of age and gender were conceptually 'held constant' through this analysis, although not experimentally.

To determine whether age-categories were similar to continuous age for OR analysis, both were calculated. Although there were no differences in the results, use of continuous age adjustment appeared to be more discriminating. Continuous age adjustment was therefore used for all further analyses. The results of both methods of age-gender adjustment are reported in Table 8.13.

Once age-gender adjustments were made, only six explanatory variables demonstrated an increased risk for periodontitis. The results of age (continuous) - gender adjustment were as follows: 1) Marital status: Those who were single had an increased risk of 1.81 times (95\% CI 1.10 2.97) compared to those with partners; 2) Ever Smoke: Those who reported ever to have smoked had an increased risk of 1.82 times (95\% CI $1.13-2.92$ ) compared to those who had never smoked; 3) \# cig/day: Those who smoked $\geq 16$ cigarettes per day had an increased risk of 2.10 (95\% CI 1.17 - 3.79) compared to those who smoked 0 - 15 per day; 4) Plaque Score: Those who had a plaque score $\geq 67 \%$ had an increased risk of 2.03 times (95\% CI 1.16 3.55 ) compared to those who had $<67 \%$ plaque score. Similarly, PS $\geq 50 \%$ had an increased OR $=1.71(95 \%$ CI $1.05-2.77)$; 5) Bleeding On Probing 
TABLE 8.13 Odds Ratios (unadjusted, age and gender-adjusted) for key explanatory
variables

\begin{tabular}{|c|c|c|c|}
\hline Variable & $\begin{array}{l}\text { Unadjusted OR } \\
(\mathbf{9 5 \%} \mathrm{CI})\end{array}$ & $\begin{array}{l}\text { Age (cat) \& gender } \\
\text { adjusted OR (95\% CI) }\end{array}$ & $\begin{array}{l}\text { Age (contin) \& gender } \\
\text { adjusted OR (95\% CI) }\end{array}$ \\
\hline \multicolumn{4}{|l|}{$\begin{array}{l}\text { Sociodemographic } \\
\text { Factors: }\end{array}$} \\
\hline Age (group) & $4.08(2.59-6.43)$ & - & \\
\hline Gender & $1.62(1.04-2.51)$ & - & \\
\hline Marital status & $1.71(1.07-2.72)$ & $1.86(1.13-3.06)$ & $1.81(1.10-2.97)$ \\
\hline Education & $1.67(1.09-2.56)$ & $1.07(0.66-1.73)$ & $1.07(0.66-1.72)$ \\
\hline Occupation & $1.56(1.21-2.95)$ & $1.29(0.66-2.54)$ & $1.29(0.66-2.51)$ \\
\hline Income & $1.31(0.85-2.0)$ & $0.98(0.61-1.58)$ & $1.08(0.68-1.71)$ \\
\hline Employment & $2.17(1.41-3.26)$ & $1.16(0.66-2.04)$ & $1.18(0.68-2.06)$ \\
\hline \multicolumn{4}{|l|}{ Behaviors: } \\
\hline Toothbrushing & $0.93(0.45-1.93)$ & $0.67(0.30-1.49)$ & $0.66(0.29-1.47)$ \\
\hline Flossing & $0.93(0.57-1.52)$ & $0.93(0.55-1.57)$ & $0.86(0.51-1.46)$ \\
\hline Dental visits & $0.62(0.40-0.97)$ & $0.70(0.43-1.14)$ & $0.70(0.43-1.13)$ \\
\hline Ever smoke & $1.64(1.07-2.51)$ & $1.73(1.08-2.77)$ & $1.82(1.13-2.92)$ \\
\hline \# Cig ( $\geq 16 /$ day $)$ & $2.0(1.16-3.44)$ & $2.20(1.21-3.99)$ & $2.10(1.17-3.79)$ \\
\hline \multicolumn{4}{|l|}{ Chronic conditions: } \\
\hline Diabetes & $2.32(1.25-6.69)$ & $1.56(0.51-4.73)$ & $1.65(0.55-4.93)$ \\
\hline Any ischem & $2.20(0.98-4.94)$ & $1.06(0.45-2.52)$ & $0.99(0.41-2.37)$ \\
\hline Diag hyper & $1.91(1.14-3.19)$ & $1.26(0.57-2.21)$ & $1.28(0.73-2.23)$ \\
\hline $\mathrm{SBP}>140$ & $1.57(0.88-2.80)$ & $0.84(0.44-1.61)$ & $0.89(0.47-1.69)$ \\
\hline $\mathrm{DBP}>90$ & $1.48(0.72-3.05)$ & $0.92(0.42-2.01)$ & $0.92(0.43-2.00)$ \\
\hline HBP combo & $1.82(1.14-2.89)$ & $1.11(0.67-1.89)$ & $1.16(0.69-1.93)$ \\
\hline \multicolumn{4}{|l|}{ Medications: } \\
\hline Anti-inflammatory & $1.83(0.91-3.67)$ & $1.34(0.64-2.83)$ & $1.35(0.64-2.84)$ \\
\hline Estrogen & $0.62(0.35-1.12)$ & $1.27(0.53-1.98)$ & $1.02(0.53-1.98)$ \\
\hline \multicolumn{4}{|l|}{ Other: } \\
\hline Stress & $0.78(0.50-1.23)$ & $1.11(0.56-1.45)$ & $0.91(0.57-1.47)$ \\
\hline \multicolumn{4}{|l|}{ Oral findings: } \\
\hline Plaque score $(67 \%)$ & $2.59(1.56-4.26)$ & $1.87(1.07-3.25)$ & $2.03(1.16-3.55)$ \\
\hline Plaque (50\%) & $1.84(1.18-2.88)$ & $1.54(0.95-2.49)$ & $1.71(1.05-2.77)$ \\
\hline Bleeding (50\%) & $2.47(1.41-4.30)$ & $2.32(1.28-4.17)$ & $2.40(1.33-4.32)$ \\
\hline \# Teeth present $<25$ & $3.17(2.03-4.94)$ & $2.03(1.23-3.33)$ & $2.06(1.26-3.39)$ \\
\hline
\end{tabular}


Score: Those who had a BOP $\geq 50 \%$ had an OR $=2.40$ (95\% CI 1.33 - 4.32); 6) \# Teeth Present: There was an increased adjusted OR $=2.06$ (95\% CI 1.26 3.39) for those with $<25$ teeth.

These results indicate that failure to adjust for age and gender would have produced misleading results that is, 'Simpson's paradox' (Simpson 1951; Rothman 1986).

\subsubsection{Age-Gender Adjusted Dose Response}

There continued to be a dose response effect after age-gender adjustment for the cigarette index (\# cig/day $\mathrm{x}$ \# years smoked). There was an increased $\mathrm{OR}=1.26, \mathrm{CI}=0.75-2.13$ for those who had a $1-300 \mathrm{cig}$. index; $\mathrm{OR}=2.14, \mathrm{CI}=0.93-4.92$, for those with $301-500$ and $\mathrm{OR}=4.36, \mathrm{CI}=$ $1.89-10.06$, for those with $>500$ compared to those with a 0 cigarette index. There was also an age - gender adjusted dose-response effect of BOP. Those with $34-66 \%$ BOP had OR $=2.22, \mathrm{CI}=1.32-3.75$, and those $\geq 67 \% \mathrm{BOP}$ had and $\mathrm{OR}=7.69, \mathrm{CI}=2.42-24.58$, compared to those with $\mathrm{BOP}$ of $0-33 \%$. There was a hint of a dose response with increasing levels of PLAQUE. The $\mathrm{OR}=1.18, \mathrm{CI}=0.67-2.07$, for those with $34-66 \%$ plaque and $\mathrm{OR}=2.24, \mathrm{CI}=$ $1.17-4.31$, for those $\geq 67 \%$ plaque compared to those with $\leq 33 \%$ plaque. Therefore, the dose response effect of plaque was not used in subsequent analyses but rather $</ \geq 50 \%$ or $</ \geq 67 \%$ plaque. The results are reported in Table 8.14. 
TABLE 8.14 Dose response to key explanatory variables: age and gender adjusted

\begin{tabular}{lll}
\hline Variable & Group & Odds Ratio (95\% CI) \\
\hline BOP & $0-33 \%$ & 1.00 \\
& $34-66 \%$ & $2.22(1.32-3.75)$ \\
& $\geq 67 \%$ & $7.69(2.41-24.58)$ \\
Cig index & 0 & 1.00 \\
(\#cig day x \#yrs smoked) & $1-300$ & $1.26(0.75-2.13)$ \\
& $301-500$ & $2.14(0.93-4.92)$ \\
& $>500$ & $4.36(1.89-10.06)$ \\
Plaque & & \\
& $0-33 \%$ & 1.00 \\
& $34-66 \%$ & $1.18(0.67-2.07)$ \\
& $\geq 67 \%$ & $2.24(1.17-4.31)$ \\
\hline
\end{tabular}

\subsection{MULTIVARIATE ANALYSES}

\subsubsection{Preliminaries To Model Building}

Logistic regression analysis has previously been proposed for analysis of individual and joint effects of a set of variables on the risk of disease in cohort studies (Cornfield et al 1961; Schlesselman, 1982). The logistic regression model may be applied to the analysis of data from casecontrol studies as well (Anderson, 1973). Logistic regression is used when the outcome is binary, such as in the current study (case vs control). The logistic transformation of the procedure forces the predicted probability to stay within the limits of $0-1$, no matter what the values of the explanatory variables are. It also permits reproduction of the sigmoidal exposure/risk curve by transforming the curve into a simpler linear relationship. This transformation allows a risk to be linked to a set of explanatory variables.

The resulting logistic model specifies that the probability of disease depends on a set of variables $x_{1}, x_{2} \ldots, x_{i}$; as in:

$$
\ln \frac{\hat{(\mathrm{p})}}{\wedge} \quad=\quad-\left[\mathrm{a}+\left(\mathrm{b}_{1} x_{1}\right)+\left(\mathrm{b}_{2} x_{2}\right)+\left(\mathrm{b}_{\mathrm{i}} x_{\mathrm{i}}\right)\right]
$$

(Kahn and Sempos 1989) 
Where $\ln$ indicates the use of the natural (base e) logarithm; $\mathrm{p} / 1-\mathrm{p}$ denotes the chance of disease divided by the chance of not having disease that is, the odds of disease; "a" indicates the intercept, which measures the $\log$ odds of disease when the explanatory variables take their zero or baseline value; "b's" represent model parameters and are therefore the log odds ratio measuring the increase or decrease in odds of disease corresponding to a one unit increase in the value of the explanatory variable after the possible influence of the other model variables has been controlled for or eliminated (Schlesselman 1982; Hassard 1992).

Multiple logistic regression analyses were employed to examine the interrelationship and association of the explanatory variables of interest to one another and with a history of periodontitis. Assessment of outliers and collinearity preceded model building.

\section{Outliers:}

Age was the only continuous variable to be used in model building. Outliers were assessed using a scatterplot. There were no outliers and age was normally distributed among the total study population $(\mathrm{n}=362)$ as well as within cases and controls.

\section{Collinearity:}

Collinearity or the high correlation between variables (Last 1988) was assessed using Chi Square cross tabs for categorical-categorical variables; linear regression for continuous-continuous variables; ANOVA for continuous - > 2 categorical variables; and t-tests for continuous - 2 category variables. Analyses were made of the OVERALL study population $(\mathrm{n}=362)$ as well as for CONTROLS $(n=154)$ alone and CASES $(n=208)$ alone in 
order to determine whether all groups experienced similar correlation patterns.

Results of the analyses indicated that there was significant correlation between a number of explanatory variables and that the pattern of correlation was consistent among the OVERALL, CONTROL and CASE groups.

AGE: Increased age was correlated with a decrease in education and income and a decrease in number of teeth present but with an increase in ischemic disease, hypertension and diabetes.

GENDER: Being male was correlated with less frequent toothbrushing and less regular visits to the dentist but with an increased history of ever smoking and increased number of cigarettes/day, cigarette index, plaque score, ischemic disease, bleeding on probing and increased income.

MARITAL STATUS: Having a partner was correlated with having a higher family income and higher bleeding on probing.

EDUCATION: Having a higher education was correlated with increased income, more frequent toothbrushing and dental visits, having $\geq 20$ teeth and $\geq 25$ teeth as well as with an increased history of ever smoking but with decreased number of cigarettes/day, cigarette index, ischemic disease, hypertension and plaque score.

TOOTHBRUSHING: More frequent toothbrushing was correlated with decreased ever smoke, number of cig/day, cigarette index, plaque but with increased dental visits and with having $\geq 20$ teeth.

INCOME: There was a trend for higher income to be correlated with decreased ischemic disease and hypertension and the presence of $\geq 20$ teeth and $\geq 25$ teeth. 
REGULAR DENTAL VISITING: There was also a trend for regular dental visiting to be correlated with decreased cigarette index, hypertension, plaque and bleeding on probing but with an increase in having $\geq 20$ teeth and $\geq 25$ teeth.

PLAQUE: A higher proportion of those with $<67 \%$ plaque have $\geq 20$ teeth.

The results of the collinearity analyses were used to select the most appropriate variables from associated pairs.

\section{Preliminary Model Parameter Decisions:}

\section{1) Stepwise Multiple Logistic Regression:}

Since assessment of collinearity suggested that the effect of one variable could be mediated by another variable, backward stepwise multiple logistic regression was used to determine the impact of specific variables on the coefficients of the remaining variables by deleting variables from the model's equation (Cramer 1972; Snedecor and Cochran 1980).

\section{2) Full Versus Reduced Models:}

The process of model building involved deciding which parameters were most informative and suitable for inclusion. Although BOP and number of teeth present appeared to be strongly associated with a history of periodontitis, there was concern that, as markers of periodontitis, they could potentially mask the effect of other explanatory variables.

\section{3) Age-Stratification:}

Since the effect of age appeared to influence many of the explanatory variables, a decision was made to stratify the total study population, 
aged $35-87$ years $(n=362)$ into two age groups, $35-54$ years $(n=184)$ and $\geq 55$ years $(n=178)$ in an attempt to determine whether the two age groups were influenced differently by the explanatory variables. A model was built for each age group:

35 - 54 year olds

$\geq 55$ year olds

4) Model Testing:

The adequacy of fit of each model was tested by two methods: 1) The likelihood ratio test was used to determine whether a variable contributed to the explanation of a history of periodontitis. 2) The goodness of fit Chi-square test was used to assess whether the final model fit the data adequately.

\section{FULL MODEL \#1 (all ages):}

Initial analysis was completed using backward stepwise logistic regression. Backward stepwise logistic regression was performed to identify the best-fitting model which described the relationship of the dependent variable and the set of explanatory variables among the total population. The initial model consisted of all variables which had, through univariate and bivariate analyses, demonstrated a significant unadjusted OR or significant dose-response effect: age (continuous), gender, marital status, employment status, regularity of dental visits, history of ever smoking, diagnosed hypertension, plaque score, number of cigarette index dose response, BOP dose response and number of teeth present. The significance level was set at 3.84. The results of the first BACKWARD step are reported in Table 8.15a.

Backward stepwise logistic regression proceeded to identify and remove the smallest Z-ratio and subsequently refit the model with the remaining main effects. Backward analysis was completed by removing 
effects one at a time until the best-fitting model was reached. No interactions were identified. The final BACKWARD model included: age, number of teeth, BOP, and Cigarette-index $>500$ as reported in Table 8.15b.

Normal multiple logistic regression was run on the FULL MODEL. The parameters selected for entry were those which resulted from backward stepwise multiple logistic regression plus age and gender (adjustment) and any required dose- response dummy variables. The beta estimates, SE, and p-values of the FULL MODEL \#1 are reported in Table 8.15c. The influence of both BOP and number of teeth present on a history of periodontitis was strong. In fact, so strong that their presence appeared to mask the effect of other explanatory variables. When BOP and number of teeth present were included in the FULL MODEL for the entire study population, only age and the cigarette-index were significant variables. In order to look more thoroughly at other potential explanatory variables, the risk markers BOP and number of teeth were eliminated from subsequent analyses of MODEL \#1.

Tables 8.15 (a - c)

Table 8.15 a - Full Model \#1 "Backward Stepwise Multiple Logistic Regression Step One

\begin{tabular}{|c|c|c|c|c|}
\hline Variable & Beta Estimate & $\overline{S E}$ & $\begin{array}{c}\text { Chi Square } \\
\text { Beta }\end{array}$ & Probability \\
\hline Intercept & -3.40 & 0.72 & 22.16 & $p<0.0001$ \\
\hline Age at $H \& E$ & 4.21 & 0.01 & 8.89 & $p<0.003$ \\
\hline Gender & 0.40 & 0.30 & 1.82 & $p=0.18$ \\
\hline Marital & 0.60 & 0.30 & 3.98 & $\mathrm{p}<0.05$ \\
\hline Employ & 0.14 & 0.34 & 0.18 & $p=0.68$ \\
\hline Appts & 0.06 & 0.29 & 0.04 & $p=0.84$ \\
\hline Smoke ever & -0.31 & 1.07 & 0.09 & $p=0.77$ \\
\hline HBP Diag & 0.07 & 0.34 & 0.04 & $p=0.84$ \\
\hline Plaque & 0.22 & 0.28 & 0.61 & $p=0.43$ \\
\hline BOP $34-66 \%$ & 0.84 & 0.31 & 7.47 & $p<0.006$ \\
\hline BOP $\geq 67 \%$ & 1.52 & 0.64 & 5.66 & $p<0.02$ \\
\hline Teeth $<25$ & 0.67 & 0.30 & 5.01 & $p<0.03$ \\
\hline Cig index $1-300$ & 0.34 & 1.03 & 0.11 & $p=0.74$ \\
\hline Cig index $301-500$ & 0.72 & 0.92 & 0.61 & $p=0.44$ \\
\hline Cig index $>500$ & 1.59 & 1.15 & 1.91 & $p=0.17$ \\
\hline
\end{tabular}


Table 8.15 b - Result of Backward Stepwise: Full Model \#1

\begin{tabular}{lllll}
\hline Intercept & -2.97 & 0.61 & 23.96 & $\mathrm{p}<0.0001$ \\
Age at H\&E & 0.05 & 0.01 & 15.58 & $\mathrm{p}<0.0001$ \\
Cig index $>500$ & 1.38 & 0.48 & 8.29 & $\mathrm{p}<0.004$ \\
Teeth $<25$ & 0.68 & 0.28 & 5.76 & $\mathrm{p}<0.02$ \\
BOP $34-66 \%$ & 1.70 & 0.61 & 7.69 & $\mathrm{p}<0.005$ \\
BOP $\geq 67 \%$ & 0.81 & 0.29 & 8.15 & $\mathrm{p}<0.004$ \\
\hline
\end{tabular}

Table 8.15 c - Result of Normal Multiple Logistic Regression: Full Model \#1

\begin{tabular}{lcccc}
\hline Intercept & -3.16 & 0.62 & 26.18 & $\mathrm{p}<0.0001$ \\
Age & 0.05 & 0.01 & 16.28 & $\mathrm{p}<0.0001$ \\
Gender & 0.29 & 0.26 & 1.20 & $\mathrm{p}=0.27$ \\
BOP 34 - 66\% & 0.72 & 0.27 & 7.04 & $\mathrm{p}<0.008$ \\
BOP $\geq 67 \%$ & 1.81 & 0.60 & 9.15 & $\mathrm{p}<0.003$ \\
\# Teeth $<25$ & 0.70 & 0.27 & 6.60 & $\mathrm{p}<0.01$ \\
Cig index $1-300$ & -0.13 & 0.28 & 0.21 & $\mathrm{p}=0.65$ \\
Cig index $301-500$ & -0.61 & 0.43 & 1.95 & $\mathrm{p}=0.16$ \\
Cig index $>500$ & 1.35 & 0.47 & 8.43 & $\mathrm{p}<0.004$ \\
\hline
\end{tabular}

\subsubsection{Reduced Model \#1:}

\section{Total Study Population:}

REDUCED MODEL \#1 was formulated using the same process as described for the FULL MODEL \#1 except that BOP and number of teeth were excluded from the model.

When BOP and number of teeth were not included in analysis, marital status and plaque were significant along with age and cigarette index. The goodness of fit Chi-square test value was $\mathrm{x}^{2}=63.89,7 \mathrm{df}, \mathrm{p}<$ 0.0001 showing that the model fit the data adequately.

The results of the first BACKWARD step, the final backward stepwise model and the beta estimates, SE, OR, CI, and p-values are reported in Tables 8.16 a-c. 
Tables 8.16 (a - c)

Table 8.16 a - Reduced model \#1 backward stepwise multiple logistic regression

Step One

\begin{tabular}{|c|c|c|c|c|}
\hline Variable & Beta Estimate & SE & $\begin{array}{c}\text { Chi Square } \\
\text { Beta }\end{array}$ & Probability \\
\hline $\begin{array}{l}\text { Intercept } \\
\text { Age at H\&E } \\
\text { Gender } \\
\text { Marital Status } \\
\text { Educ Gr } 12 \\
\text { Employment } \\
\text { Appointments } \\
\text { Smoke ever } \\
\text { \# Cig/day } \\
\text { HBP Diagnosed } \\
\text { Plaque } 50 \% \\
\text { Cig index } 1-300 \\
\text { Cig index } 301-500 \\
\text { Cig index }>500 \\
\end{array}$ & $\begin{array}{c}-3.38 \\
0.55 \\
0.35 \\
0.61 \\
-0.22 \\
0.18 \\
-0.28 \\
-0.52 \\
-0.33 \\
0.14 \\
0.42 \\
0.68 \\
1.08 \\
2.11 \\
\end{array}$ & $\begin{array}{l}0.67 \\
0.01 \\
0.28 \\
0.28 \\
0.27 \\
0.32 \\
0.27 \\
1.10 \\
0.53 \\
0.32 \\
0.26 \\
1.04 \\
0.93 \\
1.14 \\
\end{array}$ & $\begin{array}{c}25.17 \\
16.59 \\
1.60 \\
4.82 \\
0.65 \\
0.33 \\
1.08 \\
0.23 \\
0.39 \\
0.20 \\
2.57 \\
0.42 \\
1.33 \\
3.42 \\
\end{array}$ & $\begin{array}{l}p<0.0001 \\
p<0.0001 \\
p=0.21 \\
p<0.03 \\
p=0.42 \\
p=0.57 \\
p=0.30 \\
p=0.63 \\
p=0.53 \\
p=0.66 \\
p=0.11 \\
p=0.52 \\
p=0.25 \\
p=0.06\end{array}$ \\
\hline
\end{tabular}

Table 8.16 b - Result of backward stepwise: reduced model \#1

\begin{tabular}{lcccc}
\hline Intercept & -3.38 & 0.58 & 34.02 & $\mathrm{p}<0.0001$ \\
Age at H\&E & 0.57 & 0.01 & 31.43 & $\mathrm{p}<0.0001$ \\
Plaque 50\% & 0.49 & 0.25 & 3.90 & $\mathrm{p}<0.05$ \\
Marital Status & 0.57 & 0.27 & 4.58 & $\mathrm{p}<0.03$ \\
Cig index $>500$ & 1.26 & 0.43 & 8.79 & $\mathrm{p}<0.003$ \\
\hline
\end{tabular}

Table 8.16 c - Result of normal multiple logistic regression: reduced model \#1

\begin{tabular}{lcccccc}
\hline Variable & $\begin{array}{c}\text { Beta } \\
\text { Estimate }\end{array}$ & SE & $\begin{array}{c}\text { Chi } \\
\text { Square } \\
\text { Beta }\end{array}$ & p Value & OR & CI \\
\hline Intercept & -3.67 & 0.61 & 35.91 & $\mathrm{p}<0.0001$ & - & \\
Age at H\&E & 0.06 & 0.01 & 32.33 & $\mathrm{p}<0.0001$ & 1.06 & $1.04-1.08$ \\
Gender & 0.32 & 0.26 & 1.45 & $\mathrm{p}=0.23$ & 1.37 & $0.85-1.89$ \\
Marital Status & 0.57 & 0.27 & 4.63 & $\mathrm{p}<0.03$ & 1.77 & $1.24-2.30$ \\
Plaque 50\% & 0.44 & 0.25 & 3.08 & $\mathrm{p}=0.08$ & 1.55 & $1.05-2.05$ \\
Cig index 1-300 & 0.22 & 0.28 & 0.63 & $\mathrm{p}=0.43$ & 1.24 & $0.69-1.79$ \\
Cig index 301-500 & 0.58 & 0.44 & 1.75 & $\mathrm{p}=0.19$ & 1.78 & $0.91-2.66$ \\
Cig index>500 & 1.33 & 0.45 & 8.94 & $\mathrm{p}<0.003$ & 3.80 & $2.91-4.69$ \\
\hline
\end{tabular}

\section{ADJUSTED ODDS RATIOS:}

The model parameter estimates were used to calculate the $\mathrm{OR}$ and associated C.I. for each explanatory variable in REDUCED MODEL \#1.

There was an increased risk of 1.06 times with each added year for those who were $\geq 55$ compared to those who were 35 - 54 years old, CI $=1.04$ 1.08. Males had an increased OR 1.37 times that of females although the 
difference was not significant, $\mathrm{CI}=0.85-1.89$. Single individuals had an increased risk of $1.77, \mathrm{CI}=1.24-2.30$, compared to those with partners. Those with $>50 \%$ plaque had an increase risk of 1.55 times compared to those with $<50 \%, \mathrm{CI}=1.05-2.05$. There was a significant dose response effect of the cigarette index whereby those who had 1 - 300 cigarette index (i.e., \# cig/day x \# years smoked) had an increased risk of 1.24 times, those with 301 - 500 cigarette index had an increased risk of 1.78 times and those with > 500 cigarette index had an increased risk of 3.80 when compared to nonsmokers. Results are reported in Table 8.16c.

\section{Model \#1 Predictive Abilities:}

Using a subfunction of NCSS multiple logistic regression, this model was found to correctly classify $64.83 \%$ of the controls and $73.80 \%$ of the cases, accounting for $69.88 \%$ overall correct classification.

Using beta estimates for each parameter in the model, the probability of risk was calculated for various scenarios in the study population using the following formula:

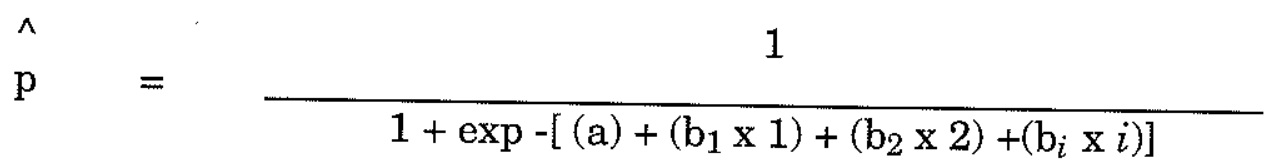

(Kahn and Sempos 1989)

\section{Scenario a:}

Male, 40 Years Old., Single, > 50\% Plaque, 0 Cig - index

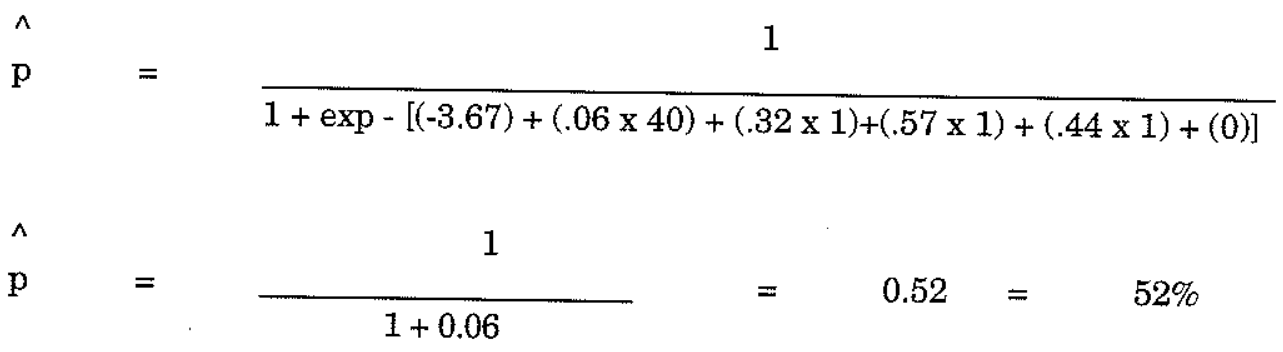


Those in the study population who fit this description have a $52 \%$ chance of being a case.

\section{Scenario b:}

Male, 65 Years Old, Married, $<50 \%$ Plaque, $0 \mathrm{Cig}$ - index $=63 \%$

\section{Scenario c:}

Female, 45 Years, Married, $>50 \%$ Plaque, 1 - 300 Cig - index $=42 \%$

\subsubsection{Age - Stratified Model Building}

\subsubsection{Full Model \# 2a}

\section{Ages 35-54 Years $(n=175)$}

Prior to building a model for the younger stratified age group, cases were compared to controls regarding exposure to explanatory variables using Chi square and t-test analyses for categorical and continuous data. In addition, un-adjusted Odds Ratios were calculated for each explanatory variable using logistic regression. Results are reported in Table 8.17.

Among those aged 35 - 54 years, there were no difference between cases and control except for the following variables: There was a higher proportion of cases $(61.8 \%)$ that had a history of ever smoking, $\mathrm{x}^{2}=4.33,1 \mathrm{df}$, $\mathrm{OR}=1.88, \mathrm{CI}=1.02-3.46, \mathrm{p}<0.04$ compared to controls. There was a significant difference in mean number of cigarette years (\# cig/day $\mathrm{x}$ \# yrs smoked) with cases experiencing a higher mean (419.83 or $153,237.95$ cigarettes) versus controls (221.14 or 80,716.10 cigarettes) $t=-3.44,95 \mathrm{df}, \mathrm{p}<$ 0.0009 . There was a higher proportion of controls with lower plaque scores compared to cases i.e., $60.8 \%$ of controls had $<50 \%$ plaque, $\mathrm{p}=0.06$ Although not being used for further analysis, similar results were found with BOP as measured by 50\% (85.0\%) and 67\% (96.0\%) $\mathrm{x}^{2}=7.15,1 \mathrm{df}, \mathrm{p}<$ 
TABLE 8.17 Explanatory variables: comparison of cases and controls (age $35-54$ )

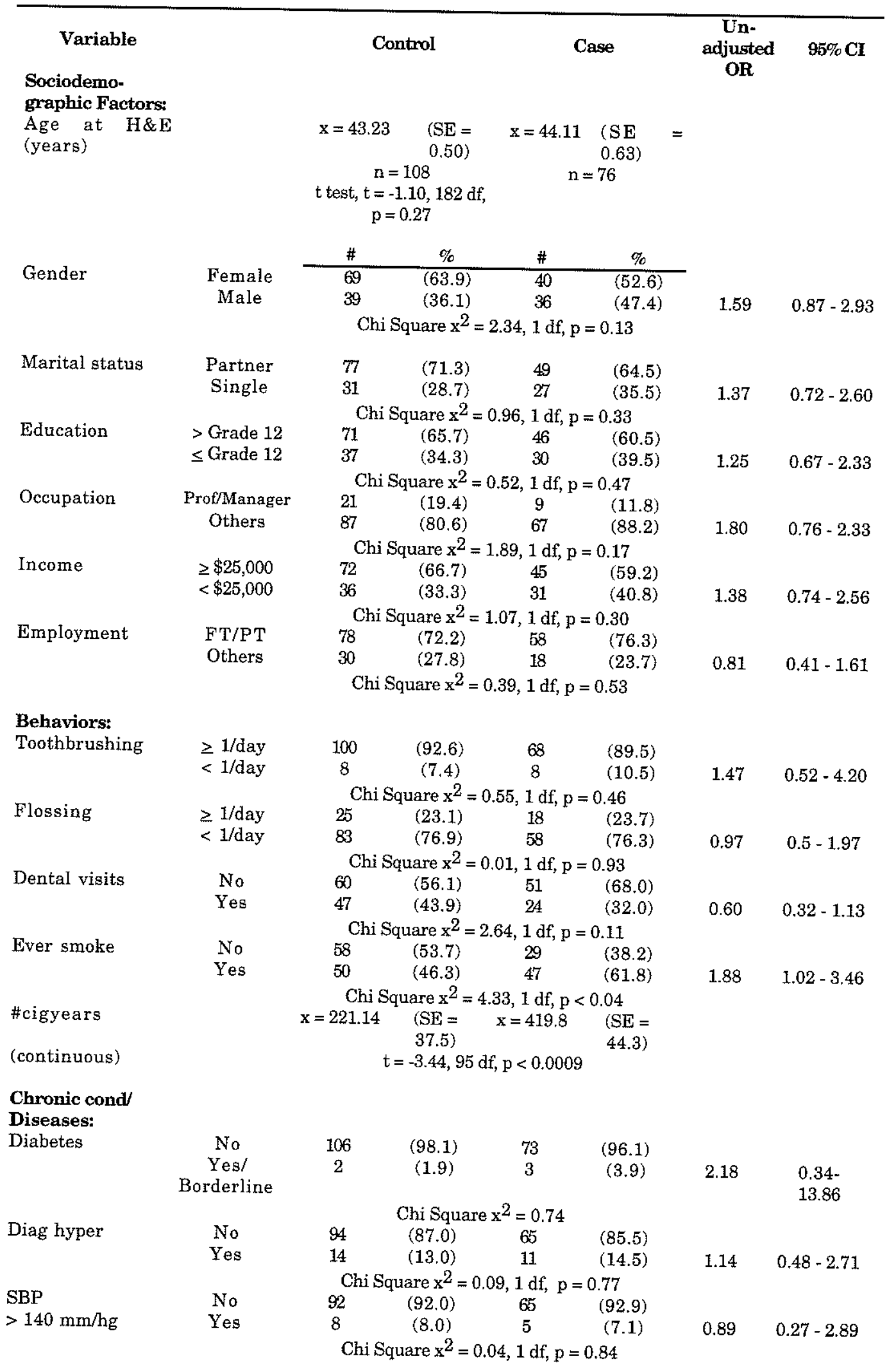




\begin{tabular}{|c|c|c|c|c|c|c|c|}
\hline $\begin{array}{l}\text { DBP } \\
>90 \mathrm{~mm} / \mathrm{hg}\end{array}$ & $\begin{array}{l}\text { No } \\
\text { Yes }\end{array}$ & $\begin{array}{l}95 \\
5\end{array}$ & $\begin{array}{c}(95.0) \\
(5.0)\end{array}$ & $\begin{array}{l}65 \\
5\end{array}$ & $\begin{array}{c}(92.9) \\
(7.1)\end{array}$ & 1.46 & $0.40-5.39$ \\
\hline Any ischemic & $\begin{array}{l}\text { No } \\
\text { Yes }\end{array}$ & $\begin{array}{c}\mathrm{C} \\
108 \\
0\end{array}$ & $\begin{array}{c}\text { uare } x^{2}= \\
(100.0) \\
(0)\end{array}$ & $\begin{array}{l}1 \mathrm{~d} \\
73 \\
3\end{array}$ & $\begin{array}{l}0.56 \\
(96.1) \\
(3.9)\end{array}$ & Cell Size & \\
\hline HBP combo & $\begin{array}{l}\text { No } \\
\text { Yes }\end{array}$ & $\begin{array}{l}88 \\
20 \\
\mathrm{Ch}\end{array}$ & $\begin{array}{c}\text { i Square } \\
(81.5) \\
(18.5) \\
\text { are } x^{2}=0\end{array}$ & $\begin{array}{c}4.33 \\
62 \\
14 \\
, 1 d\end{array}$ & $\begin{array}{l}(81.6) \\
(18.4) \\
0.99\end{array}$ & 1.01 & $0.46-2.18$ \\
\hline $\begin{array}{l}\text { Medication: } \\
\text { Anti-inflam }\end{array}$ & $\begin{array}{l}\text { No } \\
\text { Yes }\end{array}$ & $\begin{array}{l}24 \\
7\end{array}$ & $\begin{array}{l}(77.4) \\
(22.6)\end{array}$ & $\begin{array}{l}12 \\
8\end{array}$ & $\begin{array}{l}(60.0) \\
(40.0)\end{array}$ & 2.29 & $0.65-8.01$ \\
\hline Estrogen & $\begin{array}{l}\text { No } \\
\text { Yes }\end{array}$ & $\begin{array}{l}\mathrm{C} \\
15 \\
54 \\
\mathrm{C}\end{array}$ & $\begin{array}{c}\text { uare } x^{2}= \\
(21.7) \\
(78.3) \\
\text { uare } x^{2}=\end{array}$ & $\begin{array}{c}1 \mathrm{df} \\
8 \\
32 \\
1 \mathrm{df}\end{array}$ & $\begin{array}{l}0.18 \\
(20.0) \\
(80.0) \\
.83\end{array}$ & 1.11 & $0.42-2.97$ \\
\hline $\begin{array}{l}\text { Other: } \\
\text { Stress }\end{array}$ & $\begin{array}{c}\text { No/Slight } \\
\text { Mod/Severe }\end{array}$ & $\begin{array}{c}35 \\
73 \\
\mathrm{Cl}\end{array}$ & $\begin{array}{c}(32.4) \\
(67.6) \\
\text { uare } x^{2}=\end{array}$ & $\begin{array}{l}23 \\
53 \\
1 \mathrm{df}\end{array}$ & $\begin{array}{l}(30.3) \\
(69.7) \\
.76\end{array}$ & 1.11 & $0.58-2.11$ \\
\hline $\begin{array}{l}\text { Oral findings: } \\
\text { Plaque }\end{array}$ & $\begin{array}{l}<50 \% \\
\geq 50 \%\end{array}$ & $\begin{array}{l}62 \\
40 \\
\mathrm{Cl}\end{array}$ & $\begin{array}{c}(60.8) \\
(39.2) \\
\text { are } x^{2}=\end{array}$ & $\begin{array}{c}34 \\
39 \\
1 \mathrm{df},\end{array}$ & $\begin{array}{l}(46.6) \\
(53.4) \\
0.06\end{array}$ & 1.78 & $0.96-3.31$ \\
\hline Plaque & $\begin{array}{l}<67 \% \\
\geq 67 \%\end{array}$ & $\begin{array}{c}84 \\
18 \\
\mathrm{Ch}\end{array}$ & $\begin{array}{l}(82.4) \\
(17.6) \\
\operatorname{are~} x^{2}=\end{array}$ & $\begin{array}{c}47 \\
26 \\
1 \mathrm{df},\end{array}$ & $\begin{array}{l}(64.4) \\
(35.6) \\
.007\end{array}$ & 2.58 & $1.27-5.27$ \\
\hline BOP\% & $\begin{array}{l}<50 \% \\
\geq 50 \%\end{array}$ & & $\begin{array}{l}(85.0) \\
(\mathbf{1 5 . 0})\end{array}$ & $\begin{array}{l}51 \\
24\end{array}$ & $\begin{array}{l}(68.0) \\
(32.0)\end{array}$ & 2.67 & $1.26-5.63$ \\
\hline$\#$ teeth $<25$ & $\begin{array}{l}\geq 25 \\
<25\end{array}$ & $\begin{array}{l}\text { Ch } \\
91 \\
17 \\
\text { Chi }\end{array}$ & $\begin{array}{c}\operatorname{are} x^{2}= \\
(84.3) \\
(15.7) \\
\operatorname{re~}^{2}=1\end{array}$ & $\begin{array}{c}1 \mathrm{df}, \\
43 \\
33 \\
1 \mathrm{df},\end{array}$ & $\begin{array}{l}.008 \\
(56.6) \\
(43.4) \\
.0000 \\
\end{array}$ & 4.11 & $2.04-8.29$ \\
\hline
\end{tabular}


0.008 and $\mathrm{x}^{2}=7.43,1 \mathrm{df}, \mathrm{p}<0.006$. There was a significant difference between cases and controls regarding the mean number of teeth present with controls having more present (mean for controls 25.93, $\mathrm{SE}=0.31$, mean for cases $=24.37, \mathrm{SE}=0.42, \mathrm{t}=3.02,182 \mathrm{df}, \mathrm{p}<0.003$. Although there was no significant difference between cases and controls regarding number of teeth $<20, p=0.57$, there was a significant difference in number of teeth present $<25, \mathrm{x}^{2}=17.27,1 \mathrm{df}, \mathrm{OR}=4.11, \mathrm{CI}=2.04-8.29, \mathrm{p}<0.0001$. Due to inadequate numbers of exposed young subjects, differences could not be obtained for ischemic disease or for number of teeth $<10$.

\section{Dose-Response Association:}

Within the 35-54 year old age group, an unadjusted dose-response effect was investigated for plaque and BOP each using three dose categories $(0-33 \%, 34-66 \%$ and $>67 \%)$ and the cigarette index (i.e., \# cig/day $\mathrm{x} \#$ years smoked) using four dose categories (0,1-300, $301-500$, and $>500$ cigarette index). There was a significant dose-response effect for all three variables. The dose-response effects remained significant subsequent to age-gender adjustment and are also reported in Table 8.18.

\section{Age-Stratified Full Model \#2a:}

The same process of backward stepwise multiple regression followed by normal multiple logistic regression was carried out for the age-stratified FULL MODEL \#2a. The results are reported in Tables 8.19 (a-c).

In FULL MODEL \#2a, number of teeth explained a large degree of the variation between cases and controls regarding a history of periodontitis for those aged 35 - 54 years potentially masking the effect of other variables. 
TABLE 8.18 Dose response to key explanatory variables (35 - 54 year olds): reduced model \#2a

\begin{tabular}{|c|c|c|c|c|c|}
\hline & & Overall & Control & Case & Odds Ratio (95\% CI) \\
\hline Plaque unadjusted & $\begin{array}{c}0-33 \% \\
34-66 \% \\
\geq 67 \%\end{array}$ & $\begin{array}{c}57(32.6) \\
74(42.3) \\
44(25.1) \\
\quad \text { Chi Sc }\end{array}$ & $\begin{array}{r}37(64.9) \\
47(63.5) \\
18(40.9) \\
\mathrm{x}^{2}=7.33\end{array}$ & $\begin{array}{l}20(35.1) \\
27(36.5) \\
26(59.1) \\
<0.03\end{array}$ & $\begin{array}{c}1.00 \\
1.06(0.51-2.22) \\
2.67(1.17-6.11)\end{array}$ \\
\hline $\begin{array}{l}\text { Plaque age-gender } \\
\text { adjusted }\end{array}$ & $\begin{array}{c}0-33 \% \\
34-66 \% \\
\geq 67 \%\end{array}$ & $\begin{array}{c}57(32.6) \\
74(42.3) \\
44(25.1) \\
\text { Chi S }\end{array}$ & $\begin{array}{r}37(64.9) \\
47(63.5) \\
18(40.9) \\
\mathrm{x}^{2}=7.33\end{array}$ & $\begin{array}{l}20(35.1) \\
27(36.5) \\
26(59.1) \\
<0.03\end{array}$ & $\begin{array}{c}1.00 \\
1.09(0.52-2.32) \\
2.78(1.17-6.61)\end{array}$ \\
\hline BOP unadjusted & $\begin{array}{c}0-33 \% \\
34-66 \% \\
\geq 67 \%\end{array}$ & $\begin{array}{c}112(64.0) \\
47(26.9) \\
16(9.1) \\
\text { Chi Sq }\end{array}$ & $\begin{array}{c}73(65.3) \\
23(48.9) \\
4(25.0) \\
2=10.99\end{array}$ & $\begin{array}{l}39(34.8) \\
24(51.1) \\
12(75.0) \\
<0.004\end{array}$ & $\begin{array}{c}1.00 \\
1.95(0.97-3.96) \\
5.62(1.66-19.03)\end{array}$ \\
\hline $\begin{array}{l}\text { BOP age-gender } \\
\text { adjusted }\end{array}$ & $\begin{array}{c}0-33 \% \\
34-66 \% \\
\geq 67 \%\end{array}$ & $\begin{array}{c}112(64.0) \\
47(26.9) \\
16(9.1) \\
\text { Chi Sq1 }\end{array}$ & $\begin{array}{c}73(65.3) \\
23(48.9) \\
4(25.0) \\
2=10.99\end{array}$ & $\begin{array}{l}39(34.8) \\
24(51.1) \\
12(75.0) \\
<0.004\end{array}$ & $\begin{array}{c}1.00 \\
1.99(0.98-4.07) \\
5.67(1.62-19.76)\end{array}$ \\
\hline $\begin{array}{l}\text { Cig index } \\
\text { unadjusted }\end{array}$ & $\begin{array}{c}0 \\
1-300 \\
301-500 \\
>500\end{array}$ & $\begin{array}{c}87(47.3) \\
53(28.8) \\
20(10.9) \\
24(13.0) \\
\text { Chi Squ }\end{array}$ & $\begin{array}{c}58(66.7) \\
37(69.8) \\
8(40.0) \\
5(20.8) \\
=22.06,3\end{array}$ & $\begin{array}{l}29(33.3) \\
16(30.2) \\
12(60.0) \\
19(79.2) \\
0.0001\end{array}$ & $\begin{array}{c}1.00 \\
1.02(0.51-2.04) \\
3.29(1.17-9.24) \\
7.98(2.65-23.99)\end{array}$ \\
\hline $\begin{array}{l}\text { Cig index age - } \\
\text { gender adjusted }\end{array}$ & $\begin{array}{c}0 \\
1-300 \\
301-500 \\
>500\end{array}$ & $\begin{array}{c}87(47.3) \\
53(28.8) \\
20(10.9) \\
24(13.0) \\
\text { Chi Squ }\end{array}$ & $\begin{array}{c}58(66.7) \\
37(69.8) \\
8(40.0) \\
5(20.8) \\
=22.06,3\end{array}$ & $\begin{array}{l}29(33.3) \\
16(30.2) \\
12(60.0) \\
19(79.2) \\
0.0001\end{array}$ & $\begin{array}{c}1.00 \\
1.00(0.49-2.01) \\
3.15(1.11-8.96) \\
7.33(2.37-22.67)\end{array}$ \\
\hline
\end{tabular}


Tables 8.19 (a - c)

Table 8.19 a — Full Model \#2a Backward Stepwise Multiple Logistic Regression

\begin{tabular}{|c|c|c|c|c|}
\hline \multicolumn{5}{|c|}{ Step One } \\
\hline Variable & Beta Estimate & SE & $\begin{array}{c}\text { Chi Square } \\
\text { Beta }\end{array}$ & Probability \\
\hline $\begin{array}{l}\text { Intercept } \\
\text { Age at } H \& E\end{array}$ & $\begin{array}{l}-1.84 \\
0.0 ?\end{array}$ & 1.57 & 1.39 & $p=0.24$ \\
\hline Age at H\&E & 0.02 & 0.04 & 0.21 & $p=0.65$ \\
\hline Gender & 0.07 & 0.39 & 0.03 & $p=0.86$ \\
\hline Appointments & 0.08 & 0.39 & 0.00 & $\mathrm{p}=0.99$ \\
\hline Smoke ever & -0.91 & 1.40 & 0.43 & $p=0.51$ \\
\hline Plaque $\geq 67 \%$ & 0.33 & 0.46 & 0.50 & $p-0.48$ \\
\hline BOP $34-66 \&$ & 0.63 & 0.41 & 2.30 & $p=0.13$ \\
\hline $\mathrm{BOP} \geq 67 \%$ & 1.28 & 0.75 & 2.89 & $p=0.09$ \\
\hline Cig index $1-300$ & 0.49 & 1.35 & 0.13 & $p=0.71$ \\
\hline Cig index $301-500$ & 1.52 & 1.23 & 1.54 & $p=0.22$ \\
\hline Cig index $>500$ & 2.60 & 1.54 & 2.85 & $p=0.09$ \\
\hline Teeth $<25$ & 1.32 & 0.42 & 10.01 & $p<0.002$ \\
\hline
\end{tabular}

Table 8.19 b - Result of Backward Stepwise: Full Model \#2a

\begin{tabular}{lllll}
\hline Intercept & -0.90 & 0.21 & 18.18 & $\mathrm{p}<0.0001$ \\
\# Teeth $<25$ & 1.43 & 0.39 & 13.36 & $\mathrm{p}<0.003$ \\
Cig index $>500$ & 2.09 & 0.67 & 9.78 & $\mathrm{p}<0.002$ \\
\hline
\end{tabular}

Table 8.19 c — Result of Normal Multiple Logistic Regression: Full Model \#2a

\begin{tabular}{lcccc}
\hline Intercept & -0.93 & 1.40 & 0.45 & $\mathrm{p}<0.003$ \\
Age at H\&E & -0.004 & 0.03 & 0.02 & $\mathrm{p}<0.0001$ \\
Gender & 0.21 & 0.35 & 0.36 & $\mathrm{p}<0.002$ \\
Cig index $1-300$ & -0.02 & 0.37 & 0.00 & $\mathrm{p}<0.001$ \\
Cig index $301-500$ & 0.95 & 0.55 & 3.03 & $\mathrm{p}<0.02$ \\
Cig index $>500$ & 1.94 & 0.58 & 11.11 & $\mathrm{p}=0.06$ \\
\# Teeth $<25$ & 1.30 & 0.37 & 12.14 & $\mathrm{p}=0.06$ \\
\hline
\end{tabular}

\section{Components of Reduced Model \#2a:}

REDUCED MODEL \#2a was formulated in the same manner as REDUCED MODEL \#1. The variables which were entered were: age, gender, regularity of dental visiting, ever-smoke, plaque $\geq 67 \%$, and the cigarette index. BOP and number of teeth were not included in the analysis. The goodness of fit Chi-square test value was $\mathrm{x}^{2}=22.45,5 \mathrm{df}, \mathrm{p}<$ 0.0004 showing that the model fit the data adequately. Results are reported in Table 8.20 (a-c). 
Tables $8.20(a-c)$

TABLE 8.20 a — Reduced Model \#2a Backward Stepwise Multiple Logistic Regression Step One

\begin{tabular}{|c|c|c|c|c|}
\hline Variable & Beta Estimate & SE & $\begin{array}{c}\text { Chi Square } \\
\text { Beta }\end{array}$ & Probability \\
\hline Intercept & -1.88 & 1.45 & 1.69 & $p<0.01$ \\
\hline Age at $H \& E$ & 0.03 & 0.03 & 0.83 & $\mathrm{p}<0.005$ \\
\hline Gender & 0.06 & 0.36 & 0.03 & $\mathrm{p}<0.0002$ \\
\hline Appointments & -0.32 & 0.36 & 0.79 & $p<0.005$ \\
\hline Smoke ever & -1.80 & 1.33 & 1.83 & $p<0.01$ \\
\hline Plaque $\geq 67 \%$ & 0.58 & 0.41 & 2.03 & $p<0.01$ \\
\hline Cig index $1-300$ & 1.51 & 1.28 & 1.39 & $p<0.008$ \\
\hline Cig index $301-500$ & 2.33 & 1.17 & 3.95 & $p<0.02$ \\
\hline Cig index $>500$ & 3.31 & 1.41 & 5.43 & $p<0.03$ \\
\hline
\end{tabular}

TABLE $8.20 \mathrm{~b}$ - Result of Backward Multiple Logistic Regression: Reduced Model \#2a

\begin{tabular}{lllll}
\hline Intercept & -0.70 & 0.18 & 14.61 & $\mathrm{p}<0.0001$ \\
Cig index $301-500$ & 1.06 & 0.53 & 4.07 & $\mathrm{p}<0.04$ \\
Cig index $>500$ & 1.99 & 0.54 & 13.61 & $\mathrm{p}<0.0002$ \\
\hline
\end{tabular}

TABLE 8.20 c — Result of Normal Multiple Logistic Regression: Reduced Model \#2a

\begin{tabular}{lcccccc}
\hline Variable & $\begin{array}{c}\text { Beta } \\
\text { Estimate }\end{array}$ & SE & $\begin{array}{c}\text { Chi } \\
\text { Square } \\
\text { Beta }\end{array}$ & p Value & OR & CI \\
\hline Intercept & -1.20 & 1.35 & 0.78 & $\mathrm{p}=0.38$ & - & - \\
Age at H\&E & 0.0009 & 0.03 & 0.09 & $\mathrm{p}=0.77$ & 1.01 & $0.95-1.07$ \\
Gender & 0.20 & 0.33 & 0.35 & $\mathrm{p}=0.55$ & 1.22 & $0.56-1.88$ \\
Cig index $1-300$ & -0.004 & 0.35 & 0.00 & $\mathrm{p}=0.99$ & 1.00 & $0.30-1.70$ \\
Cig index $301-500$ & 1.15 & 0.52 & 4.84 & $\mathrm{p}<0.03$ & 3.15 & $2.11-4.19$ \\
Cig index $>500$ & 1.99 & 0.57 & 12.45 & $\mathrm{p}<0.0004$ & 7.33 & $6.20-8.46$ \\
\hline
\end{tabular}

\section{Adjusted Odds Ratios for REDUCED MODEL \#2a:}

The model parameter estimates observed in Table $8.20 \mathrm{c}$ were used to calculate the adjusted $\mathrm{OR}$ and associated $\mathrm{CI}$ for each explanatory variable in REDUCED MODEL \#2a. There was a significant dose response effect with the cigarette index. When those who had never smoked were compared to those with a 1 - 300 cigarette index, there was an increased risk among smokers of 1.00 (95\% CI $0.30-1.70$ ) and with a $301-500$ cigarette index, the risk was 3.15 times (95\% CI $2.11-4.19$ ) and with a > 500 cigarette index the OR $=7.33(95 \%$ CI $6.20-8.46)$. 


\section{Predictive Value of REDUCED MODEL \#2a:}

Using a subfunction of NCSS multiple logistic regression, Model \#2a was found to correctly classify $88.89 \%$ of the controls and $39.47 \%$ of the cases, accounting for $68.48 \%$ overall correct classification.

Using beta estimates for each parameter in the model and the formula described above, the probability of risk was calculated for various scenarios in the study population:

\section{Scenario a:}

Male, 50 Years, $>500$ Cig index $=74 \%$

\section{Scenario b:}

Male, 50 Years, 0 Cig index $=28 \%$

Scenario c:

Female, 50 Years, $>500 \mathrm{Cig}$ index $=70 \%$

Scenario d:

Female, 35 Years, 0 Cig index $=25 \%$

\subsubsection{Model \#2b}

\section{Ages $\geq 55$ Years $(n=164)$}

Prior to building a model for the older age group, cases were compared to controls regarding exposure to explanatory variables. Chi square and t-test analyses for categorical and continuous data. In addition, un-adjusted Odds Ratios were calculated for each explanatory variable using logistic regression. Results are reported in Table 8.21.

Among the $\geq 55$ year age group, there were no differences between cases and controls, with the following exceptions: There was a significantly higher proportion $(80.4 \%)$ of individuals with partners among control compared to cases, $\mathrm{x}^{2}=5.55,1 \mathrm{df}, \mathrm{OR}=2.59, \mathrm{CI}=1.13-5.91$. A trend was observed for a higher proportion of controls $(71.7 \%)$ to have $<\$ 25,000$ income compared to cases, $\mathrm{x}^{2}=2.88,1 \mathrm{df}, \mathrm{p}=0.09, \mathrm{OR}=0.54, \mathrm{CI}=0.25-1.13$. There was also a trend for a higher proportion (63.0\%) of never smokers to be 
TABLE 8.21 Explanatory variables: comparison of cases and controls (age $\geq 55$ years)

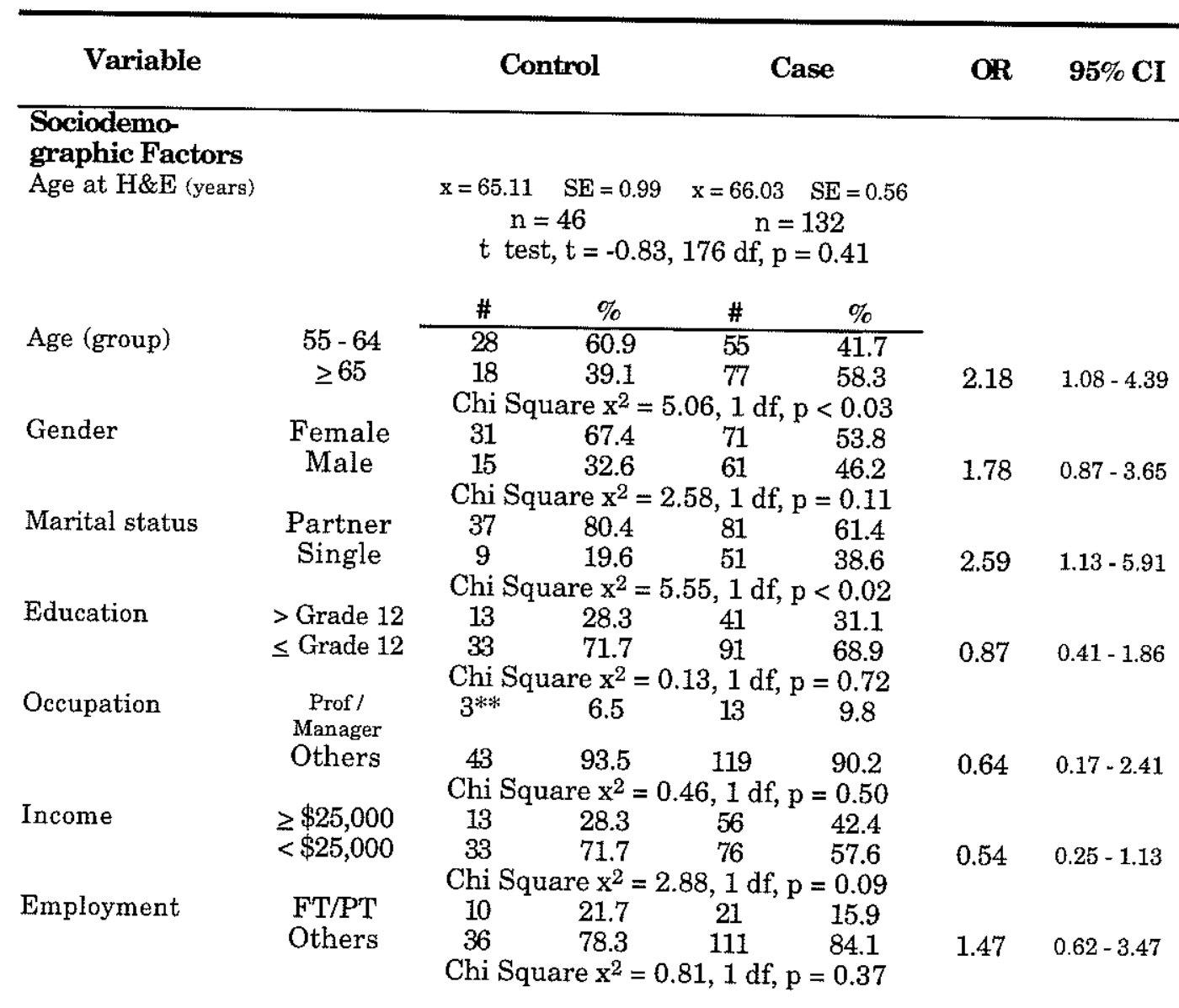

Behaviors:

Toothbrushin

Flossing

Dental visits

Ever smoke

\#CigYears

(continuous)

$$
\begin{aligned}
& \geq 1 / \text { day } \\
& <1 / \text { day }
\end{aligned}
$$$$
\geq 1 / \text { day }
$$

No

No
Yes

Chronic cond / diseases:

Diabetes

$$
\begin{aligned}
& \begin{array}{llll}
39 & 84.8 & 121 & 91.7
\end{array}
\end{aligned}
$$

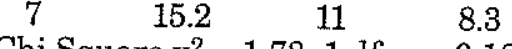

$$
\begin{aligned}
& \text { Chi Square } x^{2}=1.78,1 \mathrm{df}, \mathrm{p}=0.18 \\
& \begin{array}{llll}
13 & 28.3 & 36 & 27.3
\end{array} \\
& \text { Chi Square } x^{2}=0.02,1 \mathrm{df}, \mathrm{p}=0.90 \\
& \begin{array}{llll}
29 & 65.9 & 91 & 71.1 \\
15 & 34.1 & 37 & 28.9
\end{array} \\
& \text { Chi Square } \mathrm{x}^{2}=0.42,1 \mathrm{df}, \mathrm{p}=0.52 \\
& \begin{array}{llll}
29 & 63.0 & 63 & 47.7
\end{array} \\
& \begin{array}{llll}
17 & 37.0 & 69 & 52.3
\end{array} \\
& \begin{array}{c}
\text { Chi Square } x^{2}=3.21,1 d f, p=0.07 \\
x=442.35 \quad S E=122.21 \quad x=434.06 \quad S E=77.12
\end{array} \\
& \mathrm{t}=0.049,84 \mathrm{df}, \mathrm{p}=0.90
\end{aligned}
$$

$0.51 \quad 0.18-1.43$

$1.05 \quad 0.49-2.25$

$0.79 \quad 0.37-1.66$

$1.87 \quad 0.93-3.78$

$$
\begin{gathered}
\text { No } \\
\text { Yes / } \\
\text { Borderline }
\end{gathered}
$$

$\begin{array}{cccc}43 & 93.5 & 120 & 90.9 \\ 3 & 6.5 & 12 & 9.1\end{array}$

90.9
9.1

$1.43 \quad 0.38-5.47$

Chi Square $\mathrm{x}^{2}=0.29,1 \mathrm{df}, \mathrm{p}=0.59$ 


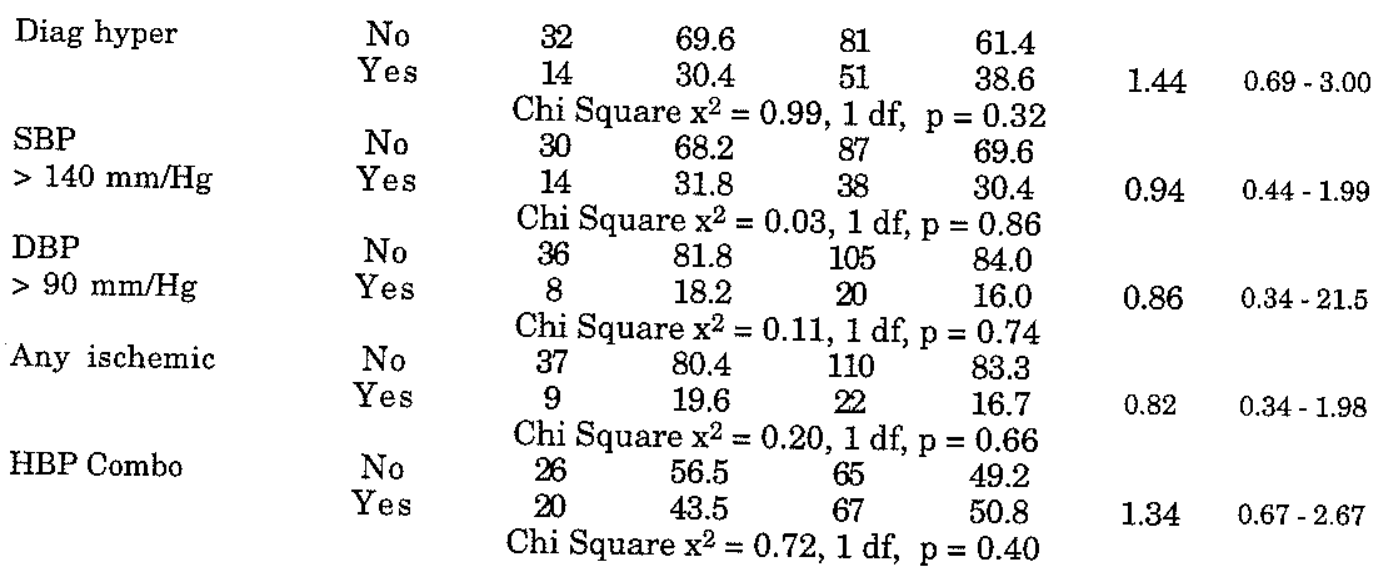

\section{Medication:}

Anti-

inflammatory

Estrogen

\begin{tabular}{|c|c|c|c|c|}
\hline No & 11 & 42.3 & 28 & 42.4 \\
\hline Yes & $\begin{array}{c}15 \\
\text { Chi Square }\end{array}$ & $\begin{array}{l}57.7 \\
x^{2}=\end{array}$ & $\begin{array}{r}38 \\
01,1\end{array}$ & $\begin{array}{c}57.6 \\
p=0.99\end{array}$ \\
\hline $\begin{array}{l}\text { No } \\
\text { Yes }\end{array}$ & $\begin{array}{l}14 \\
17\end{array}$ & $\begin{array}{l}45.2 \\
54.8\end{array}$ & $\begin{array}{l}36 \\
35\end{array}$ & $\begin{array}{l}50.7 \\
49.3\end{array}$ \\
\hline
\end{tabular}

Chi Square $\mathrm{x}^{2}=0.27,1 \mathrm{df}, \mathrm{p}=0.61$

Other:

Stress

$\begin{array}{lllll}\text { No / Slight } & 15 & 32.6 & 56 & 42.4\end{array}$ $\begin{array}{lllll}\text { Moderate } & 31 & 67.4 & 76 & 57.6\end{array}$

/ Severe

Chi Square $x^{2}=1.37,1 \mathrm{df}, p=0.24$

\section{Oral findings:}

Plaque 50\%

Plaque 67\%

BOP

\#Teeth $<10$

\#Teeth $<20$

$\#$ Teeth $<25$

$<50 \%$

$\geq 50 \%$

$<67 \%$

$\geq 67 \%$

$<50 \%$

$\geq 50 \%$

$\geq 10$

$<10$

$\geq 20$

$<20$

2

$\begin{array}{llll}22 & 51.2 & 46 & 40.4\end{array}$

Chi Square $x^{2}=1.07,1$ df $p=0.30$

$32 \quad 74.4 \quad 75 \quad \mathrm{p}=65.8$

$\begin{array}{llll}11 & 25.6 & 39 & 34.2\end{array}$

Chi Square $\mathrm{x}^{2}=7.30,1 \mathrm{df}, \mathrm{p}<0.007$

$\begin{array}{llll}34 & 82.9 & 85 & 69.1\end{array}$

$\begin{array}{llll}7 & 17.1 & 38 & 30.9\end{array}$

Chi Square $\mathrm{x}^{2}=2.95,1 \mathrm{df}, \mathrm{p}=0.008$

$\begin{array}{llll}41 & 89.1 & 126 & 95.5\end{array}$

Chi Square $\mathrm{x}^{2}=2.35,1 \mathrm{df}=0.5$

$27 \begin{array}{lll}58.7 & 95 & 72.0\end{array}$

$\begin{array}{llll}19 & 41.3 & 37 & 28.0\end{array}$

Chi Square $\mathrm{x}^{2}=2.79,1 \mathrm{df}, \mathrm{p}=0.09$

$\geq 25$
$<25$

$\begin{array}{llll}12 & 26.1 & 38 & 28.8\end{array}$

Chi Square $\mathrm{x}^{2}=0.12,1 \mathrm{df}, \mathrm{p}=0.73$

$1.55 \quad 0.75-3.18$

$1.51 \quad 0.68-3.38$

$2.17 \quad 0.87-5.43$

$0.39 \quad 0.11 \cdot 1.38$

$0.55 \quad 0.27-1.13$

$0.87 \quad 0.40-1.89$ 
controls compared to cases, $\mathrm{x}^{2}=3.21,1 \mathrm{df}, \mathrm{p}=0.07$, OR $=1.87, \mathrm{CI}=0.93$ 3.78. Although not included in subsequent analyses, there was a trend for a greater proportion (82.9\%) of controls to have a BOP $<50 \%$ compared to cases, $\mathrm{x}^{2}=2.95,1 \mathrm{df}, \mathrm{p}=0.08$ and there was a trend for a greater proportion $(72.0 \%)$ of cases to have $\geq 20$ teeth compared to controls, $x^{2}=2.79,1 \mathrm{df}, \mathrm{p}=$ 0.09 although there was no difference between them regarding $<25$ teeth.

\section{Dose-Response Association:}

Among those aged $\geq 55$ years old, an un-adjusted and adjusted doseresponse effect was investigated for plaque and BOP each using three dose categories $(0-33 \%, 34-66 \%$, and $>67 \%)$ and the cigarette index (i.e., \# cig/day $\mathrm{x}$ \# years smoked) and four dose categories (0,1 - 300, 301 - 500, > $500)$. There was not a significant dose response effect for any of the unadjusted or adjusted variables. Cell size was inadequate to investigate BOP dose response. Results are reported in Table 8.22.

\section{Age-Stratified Full Model \#2b}

In FULL MODEL \# 2b, neither BOP nor number of teeth appeared to directly influence a history of periodontitis. The results of analysis are reported in Table 8.23 (a-c).

Tables $8.23(a-c)$

TABLE 8.23 a - Full Model \#2b Backward Stepwise Multiple Logistic Regression Step One

\begin{tabular}{|c|c|c|c|c|}
\hline Variable & Beta Estimate & SE & $\begin{array}{c}\text { Chí Square } \\
\text { Beta }\end{array}$ & Probability \\
\hline Intercept & -1.19 & 2.10 & 0.32 & $p=0.57$ \\
\hline Age at $\mathrm{H} \& \mathrm{E}$ & 0.03 & 0.03 & 0.64 & $p=0.42$ \\
\hline Gender & 0.59 & 0.44 & 1.83 & $p=0.18$ \\
\hline Marital Status & 0.97 & 0.45 & 4.70 & $p<0.03$ \\
\hline Income $<\$ 25,000$ & -0.52 & 0.44 & 1.45 & $p=0.23$ \\
\hline Brush < 1/day & -0.52 & 0.64 & 0.67 & $p=0.41$ \\
\hline Smoke ever & 0.54 & 0.40 & 1.77 & $p=0.18$ \\
\hline$B O P \geq 50 \%$ & 0.74 & 0.48 & 2.40 & $p=0.12$ \\
\hline Teeth $<25$ & 0.12 & 0.45 & 0.07 & $p=0.78$ \\
\hline
\end{tabular}


TABLE 8.22 Dose response to key explanatory variables ( $\geq 55$ year olds): reduced model $\# 2 \mathrm{~b}$

\begin{tabular}{|c|c|c|c|c|c|}
\hline & & Overall & Control & Case & $\begin{array}{c}\text { Odds Ratio } \\
\text { (95\% CI) }\end{array}$ \\
\hline Plaque unadjusted & $\begin{array}{c}0-33 \% \\
34-66 \% \\
\geq 67 \%\end{array}$ & $\begin{array}{r}42(26.8) \\
65(41.4) \\
50(31.8) \\
\mathrm{Ch}\end{array}$ & $\begin{array}{c}14(33.3) \\
18(27.7) \\
11(22.0) \\
x^{2}=1.48,2\end{array}$ & $\begin{array}{l}28(66.7) \\
47(72.3) \\
39(78.0) \\
48\end{array}$ & $\begin{array}{c}1.00 \\
1.21(0.53-2.80) \\
1.83(0.85-3.94)\end{array}$ \\
\hline $\begin{array}{l}\text { Plaque age-gender } \\
\text { adjusted }\end{array}$ & $\begin{array}{c}0-33 \% \\
34-66 \% \\
\geq 67 \%\end{array}$ & $\begin{array}{r}(\mathbf{n}=157) \\
42(26.8) \\
65(41.4) \\
50(31.8) \\
\text { Ch }\end{array}$ & $\begin{array}{c}(n=43) \\
14(33.3) \\
18(27.7) \\
11(22.0) \\
x^{2}=1.48,2\end{array}$ & $\begin{array}{l}(\mathrm{n}=114) \\
28(66.7) \\
47(72.3) \\
39(78.0) \\
48\end{array}$ & $\begin{array}{c}1.00 \\
1.28(0.53-3.07) \\
1.57(0.58-4.25)\end{array}$ \\
\hline BOP unadjusted & $\begin{array}{c}0-33 \% \\
34-66 \% \\
\geq 67 \%\end{array}$ & $\begin{array}{c}(\mathrm{n}=164) \\
83(50.6) \\
70(42.7) \\
11(6.7) \\
\mathrm{Ch}\end{array}$ & $\begin{array}{c}(\mathrm{n}=41) \\
28(33.7) \\
13(18.6) \\
0(0) \\
\mathrm{x}^{2}=8.59,2\end{array}$ & $\begin{array}{c}(\mathrm{n}=123) \\
55(66.3) \\
57(81.4) \\
11(100.0) \\
.01\end{array}$ & Cell size \\
\hline $\begin{array}{l}\text { BOP age-gender } \\
\text { adjusted }\end{array}$ & $\begin{array}{c}0-33 \% \\
34-66 \% \\
\geq 67 \%\end{array}$ & $\begin{array}{c}(\mathrm{n}=164) \\
83(50.6) \\
70(42.7) \\
11(6.7) \\
\mathrm{Ch}\end{array}$ & $\begin{array}{c}(\mathrm{n}=41) \\
28(33.7) \\
13(18.6) \\
0^{* *}(0) \\
\mathrm{x}^{2}=8.59,\end{array}$ & $\begin{array}{c}(\mathrm{n}=123) \\
55(66.3) \\
57(81.4) \\
11(100.0) \\
.01\end{array}$ & Cell size \\
\hline $\begin{array}{l}\text { Cig index } \\
\text { unadjusted }\end{array}$ & $\begin{array}{c}0 \\
1-300 \\
301-500 \\
>500\end{array}$ & $\begin{array}{c}(\mathrm{n}=178) \\
92(51.7) \\
45(25.3) \\
16(9.0) \\
25(14.0) \\
\mathrm{Ch}\end{array}$ & $\begin{array}{c}(n=46) \\
29(31.5) \\
9(20.00 \\
4(25.0) \\
4(16.0) \\
x^{2}=3.62\end{array}$ & $\begin{array}{l}(\mathrm{n}=132) \\
63(68.5) \\
36(80.0) \\
12(75.0) \\
21(84.0) \\
31\end{array}$ & $\begin{array}{c}1.00 \\
1.69(0.73-3.88) \\
1.15(0.33-3.99) \\
2.34(0.72-7.62)\end{array}$ \\
\hline $\begin{array}{l}\text { Cig index age - gender } \\
\text { adjusted }\end{array}$ & $\begin{array}{c}0 \\
1-300 \\
301-500 \\
>500\end{array}$ & $\begin{array}{c}(\mathrm{n}=178) \\
92(51.7) \\
45(25.3) \\
16(9.0) \\
25(14.0) \\
\quad \mathrm{Ch}\end{array}$ & $\begin{array}{c}(\mathbf{n}=46) \\
29(31.5) \\
9(20.00 \\
4(25.0) \\
4(16.0) \\
x^{2}=3.62,3\end{array}$ & $\begin{array}{l}(\mathrm{n}=132) \\
63(68.5) \\
36(80.0) \\
12(75.0) \\
21(84.0) \\
31\end{array}$ & $\begin{array}{c}1.00 \\
1.66(0.71-3.89) \\
1.11(0.01-3.99) \\
2.23(0.01-7.58)\end{array}$ \\
\hline
\end{tabular}


TABLE 8.23 b - Result of Backward Stepwise: Full Model \#2b

\begin{tabular}{lllll}
\hline Intercept & 0.52 & 0.27 & 3.86 & $\mathrm{p}<0.05$ \\
Marital Status & 0.91 & 0.43 & 4.51 & $\mathrm{p}<0.03$ \\
Gender & 0.78 & 0.39 & 3.99 & $\mathrm{p}<0.05$ \\
\hline
\end{tabular}

TABLE 8.23 c - Result of Normal Multiple Logistic Regression: Full Model \#2b

\begin{tabular}{lcccc}
\hline Intercept & -0.22 & 1.85 & 0.01 & $p=0.90$ \\
Age at H\&E & 0.01 & 0.03 & 0.14 & $p=0.71$ \\
Gender & 0.70 & 0.37 & 3.58 & $p=0.06$ \\
Marital Status & 1.05 & 0.42 & 6.14 & $p<0.01$ \\
\hline
\end{tabular}

\section{Components Of Reduced Model \#2b:}

Backward stepwise logistic regression was performed to identify the best-fitting model which described the relationship of the dependent variable and a set of explanatory variables for the age group $\geq 55$ years.

The initial model for the $\geq 55$ year age group consisted of all variables which had demonstrated either a trend or significant unadjusted or agegender adjusted OR. There were no dose response effects entered as none were significant in preliminary analyses. The model included: age, gender, marital status, income, toothbrushing and history of ever smoking.

Backward stepwise logistic regression identified and eliminated the smallest Z-values. The final model contained all significant parameters plus age and gender for adjustment purposes. No interactions were observed. A summary of the results are reported in Table 8.24 (a-c)

Tables $8.24(a-c)$

Table 8.24 a - Reduced Model \#2b Backward Stepwise Multiple Logistic Regression Step One

\begin{tabular}{|c|c|c|c|c|}
\hline Variable & Beta Estimate & SE & $\begin{array}{c}\text { Chi Square } \\
\text { Beta }\end{array}$ & Probability \\
\hline $\begin{array}{l}\text { Intercept } \\
\text { Age at H\&E } \\
\text { Gender } \\
\text { Marital Status } \\
\text { Income }<\$ 25,000 \\
\text { Brush }<1 / \text { day } \\
\text { Smoke ever } \\
\end{array}$ & $\begin{array}{c}-1.01 \\
0.03 \\
0.55 \\
1.07 \\
-0.56 \\
-0.77 \\
0.64 \\
\end{array}$ & $\begin{array}{l}1.99 \\
0.03 \\
0.42 \\
0.43 \\
0.40 \\
0.58 \\
0.39 \\
\end{array}$ & $\begin{array}{l}0.26 \\
0.75 \\
1.71 \\
6.15 \\
1.97 \\
1.76 \\
2.64 \\
\end{array}$ & $\begin{array}{l}p=0.61 \\
p=0.39 \\
p=0.19 \\
p<0.01 \\
p=0.16 \\
p=0.19 \\
p=0.10\end{array}$ \\
\hline
\end{tabular}


Table 8.24 b - Result of Backward Stepwise Multiple Logistic Regression: Reduced Model \#2b

\begin{tabular}{lllll}
\hline Intercept & 1.25 & 0.32 & 15.48 & $\mathrm{p}<0.0001$ \\
Marital Status & 1.07 & 0.42 & 6.43 & $\mathrm{p}<0.01$ \\
Income & -0.77 & 0.38 & 4.05 & $\mathrm{p}<0.04$ \\
\hline
\end{tabular}

Table 8.24 c — Result of Normal Multiple Logistic Regression: Reduced Model \#2b

\begin{tabular}{lcccccc}
\hline Variable & $\begin{array}{c}\text { Beta } \\
\text { Estimate }\end{array}$ & SE & $\begin{array}{c}\text { Chi } \\
\text { Square } \\
\text { Beta }\end{array}$ & p Value & OR & 95\% CI \\
\hline Intercept & 0.08 & 1.87 & 0.00 & $\mathrm{p}=0.97$ & - & - \\
Age at H\&E & 0.01 & 0.03 & 0.21 & $\mathrm{p}=0.65$ & 1.01 & $0.95-1.07$ \\
Gender & 0.57 & 0.38 & 2.27 & $\mathrm{p}=0.13$ & 1.78 & $1.02-2.54$ \\
Marital Status & 1.12 & 0.43 & 6.89 & $\mathrm{p}<0.008$ & 3.07 & $2.22-3.9$ \\
Income $<\$ 25,000$ & -0.66 & 0.39 & 2.82 & $\mathrm{p}=0.09$ & 0.52 & $-0.26-1.3$ \\
\hline
\end{tabular}

\section{Adjusted Odds Ratios for REDUCED MODEL \#2b:}

Model parameter estimates and SE were used to calculate the OR and associated CI for each explanatory variable in the model. Among the $\geq 55$ year old group, there was an increased risk for those who were single versus those with a partner, $\mathrm{OR}=3.07, \mathrm{CI}=2.22-3.93$. There was a trend for increased risk for those with $\geq \$ 25,000 /$ year family income, $\mathrm{OR}=0.52, \mathrm{CI}$ $=-0.26-1.30$. The odds ratios, CIs and $\mathrm{p}$ values are reported in Table 8.24c.

\section{Predictive Value of REDUCED MODEL \#2b:}

Using a subfunction of NCSS Multiple Logistic Regression, Model \#2b was found to correctly classify $0 \%$ of controls but $100 \%$ of cases, accounting for $74.16 \%$ overall correct classification.

Beta estimates were used with the formula described above to calculate the probability of risk for various scenarios in the study population.

Scenario a:

Male, 55 Years, Married, $<\$ 25,000=63 \%$

Scenario b:

Male, 55 Years, Single, $\geq \$ 25,000=92 \%$ 


\section{Scenario c:}

Female, 80 Years, Single $\geq \$ 25,000=88 \%$

Scenario d:

Female, 80 Years, Single, $<\$ 25,000=79 \%$

All multivariate analyses were performed using the NCSS statistical software program. 


\section{CHAPTER NINE \\ DISCUSSION AND CONCLUSIONS}

\subsection{OVERALL FINDINGS}

Chapter eight described three models which were used to analyze the influence of a number of explanatory variables on the history of periodontitis. Model \#1 included the total study population (35 - 87 year olds) while Models \#2a and \#2b included two age-subgroups (35 - 54 years) and ( $\geq 55$ years) respectively. Explanatory variables initially included in the models were selected through univariate, bivariate and multivariate statistical tests. The parameters for each final model are presented in Table 9.1 .

TABLE 9.1 - Model parameters for final models

\begin{tabular}{|c|c|c|c|c|c|c|c|c|}
\hline \multicolumn{3}{|c|}{$\begin{array}{c}\text { Total Study Population } \\
35 \text { - } 87 \text { year old }\end{array}$} & \multicolumn{3}{|c|}{$\begin{array}{l}\text { Young Adults } \\
\text { 35-54 years old }\end{array}$} & \multicolumn{3}{|c|}{$\begin{array}{l}\text { Older Adults } \\
\geq 55 \text { years old }\end{array}$} \\
\hline \multirow{8}{*}{$\begin{array}{l}\text { Age } \\
\text { Gender } \\
\text { Marital } \\
\text { Plaque } \\
\text { Cig index } \\
\text { (1-300) } \\
\text { Cig index } \\
(301-500) \\
\text { Cig index } \\
(>500)\end{array}$} & $\mathbf{O R}$ & p-value & & OR & p-value & & OR & p-value \\
\hline & $\begin{array}{l}1.06 \\
1.37\end{array}$ & $\begin{array}{l}p<0.0001 \\
p=023\end{array}$ & Age & 1.01 & $p=0.77$ & Age & 1.01 & $\begin{array}{l}p=0.65 \\
p=0.13\end{array}$ \\
\hline & 1.77 & $p<0.03$ & Gender & 1.22 & $p=0.55$ & Gender & $\begin{array}{l}1.78 \\
3.07\end{array}$ & $\begin{array}{l}p=0.13 \\
p<0.008\end{array}$ \\
\hline & 1.55 & $p=0.08$ & & & & & & \\
\hline & 1.24 & $p=0.43$ & $\begin{array}{l}\text { Cig index } \\
(1-300)\end{array}$ & 1.00 & $p=0.99$ & & & \\
\hline & 1.78 & $p=0.19$ & $\begin{array}{l}\text { Cig index } \\
(301-500)\end{array}$ & 3.15 & $p<0.03$ & & & \\
\hline & 3.80 & $p<0.003$ & $\begin{array}{l}\text { Cig index } \\
(>500)\end{array}$ & 7.33 & $p<0.0004$ & & & \\
\hline & & & & & & Income & 0.52 & $p=0.09$ \\
\hline
\end{tabular}

Age and gender were forced into all three models to adjust for their confounding influences. Marital status, specifically being single, was significantly associated with an increased risk of a history of AP in the total study population as well as within the older adults but marital status did not influence younger adults. While having a plaque score $>50 \%$ at the time of the initial periodontal exam appeared to slightly increase risk of a history of AP among the total population, this trend did not apply to either of the 
age-stratified groups. The dose-response effect of the cigarette index was evident among the total population and even more so among the young adults. However, there was no evidence of an effect of cigarette smoking on AP among those $\geq 55$ years. Annual family income $<\$ 25,000$ appeared to have a protective influence on a history of AP among older adults but had no influence on the total study population or those 35 - 54 years.

This chapter discusses the role of the variables present in each agestratified model as well as pertinent variables which, although significant in other studies or in the current study's univariate or bivariate analyses did not maintain their effect in the presence of the other variables.

Due to the strong influence of age on explanatory variables, particular attention was given to the relevance of the variables found in the two age-stratified models.

\subsection{EXPLANATORY VARIABLES}

\subsubsection{Sociodemographic Factors}

The demographic variables which were strongly associated with AP in univariate and bivariate analyses were age and gender.

\subsubsection{Age}

In the current study, univariate analyses showed that being $\geq 55$ years was associated with being four times more likely to have a history of AP than those $<55$ years. This finding is consistent with other crosssectional studies (Baelum et al 1986; Miller et al 1987 Papapanou et al 1988) as well as with longitudinal studies regarding the effect of increased age on AP (Grbic et al 1991; Haffajee et al 1993).

Until recently, such results were interpreted as evidence of a greater prevalence/severity of AP among the aged (van der Velden 1994). However, 
such studies failed to recognize that periodontitis is a cumulative destructive process over an individual's lifetime. The apparent increased prevalence among the elderly compared to younger adults more likely reflects their cumulative, life-time experience with periodontal disease.

Rather than an "age-effect", increased prevalence of a history of AP among the elderly has also been explained by a "cohort-effect", that is, the 'old elderly' cohort likely have different beliefs than do the 'young elderly' regarding dental health values and knowledge. Differences may be attributed to growing up when dentistry was a luxury, when prevention was less emphasized and extraction for AP was standard practice (Walden 1988; Ettinger 1992). Differences in values and knowledge influence behavior of the various age-cohorts and may partially explain the oral health variations observed between younger adults and the elderly.

Recent studies suggest that age is a correlate rather than a risk factor for periodontitis (Abdellatif and Burt 1987; Burt 1994). In fact, AP was not inevitable with age when plaque was adequately controlled (Ismail and Lewis 1993). The $\mathrm{OR}=1.24$ for the association of age with periodontitis was relatively weak in a cross-sectional study while the OR for plaque was 20.52 (Abdellatif and Burt 1987).

Age is perhaps best considered a determinant of a history of periodontitis. Increased age has been associated with poor oral hygiene (Payne and Locker 1992) and with lower education/ income, all known to be associated negatively with regularity of dental visiting and oral self-care as well as periodontal health and tooth retention (Miller et al 1987; Walden 1988; Haffajee et al 1993).

The current study showed that age was correlated with a number of explanatory variables. Being $\geq 55$ years was positively related to having $\leq$ Grade 12 education and $<\$ 25,000$ annual family income. Many of the 
elderly had less education and/or were retired, perhaps explaining their lower income. In the overall study population, those with > Grade 12 education were positively associated with $<50 \%$ plaque scores due most likely to a higher level of health awareness and preventive behavior. Those with $\geq 25$ teeth were positively associated with having $>$ Grade 12 education, $\geq \$ 25,000$ annual family income, and more regular dental visits.

Through univariate analyses, the present study showed that a history of periodontitis was associated with lower levels of education and employment, $\mathrm{OR}=1.67$ and $\mathrm{OR}=2.17$ respectively. However, once agegender adjusted, these variables were no longer significant suggesting that the older subjects were less educated and not currently employed. Although age does not directly cause periodontitis, it is clear that its confounding or interactive effects must be controlled in periodontal research.

Today's 'new elderly' are retaining more of their teeth, due in part to their increased education and income along with access to improved prevention and treatment. As this trend continues, dental visiting rates and preventive practices among the elderly will probably increase, equalling those of other age groups (Beck and Hunt 1985; Walden 1988). After all, being dentate is one of the best predictors of dental visiting (Locker and Leake 1993). The current study showed a correlation between having $\geq$ 25 teeth present and regularity of dental visiting. Edentulous and partially edentulous individuals often fail to perceive a reason for seeking dental care unless they are in pain.

The current study ultimately analyzed the influence of a set of explanatory variables on a history of periodontitis. The effect of age was controlled by including age in the final model, and further by agestratification. 
Model \#1 showed a significantly increased risk within the total study population of 1.06 times with each increasing year for those who were older ( $\geq 55 \mathrm{yrs}$ ) compared to those who were younger (35 - $54 \mathrm{yrs}$ ).

Model \#2a showed an increased risk within the younger adults, OR = 1.01 for those aged 45 - 54 years compared to those aged 35 - 44 years. The OR however was not significant.

Model \#2b also showed an insignificant increased risk within the older adults, $\mathrm{OR}=1.01$ for those aged $\geq 65$ years compared to those aged 55 64 years.

The odds ratio for each model is somewhat different, with the stratified models showing a lower (insignificant) OR than the total model because the stratified models adjusted or controlled for age through their design. Once controlled, age did not uniquely influence periodontitis substantially in any of the models because much of the variation in the models was explained by other variables.

\subsubsection{Gender}

In the current study, univariate analysis showed that being male had an un-adjusted $\mathrm{OR}=1.62, \mathrm{CI}(1.04-2.51)$, that is, males were at 1.62 times greater risk for a history of AP than females. This could be due to poorer oral hygiene and less regular dental visiting among the males. Being male was correlated with less frequent toothbrushing and less regular dental visiting in the current study. Males also showed a greater history of ever smoking, number of cigarettes/day, a higher cigarette index, plaque score, and BOP score.

These results are consistent with earlier studies which show that males had higher levels of plaque (Grbic et al 1991; Haffajee et al 1993), and 
a greater prevalence/severity of AP (Baelum et al 1986; Miller et al 1987; Hunt et al 1990) although the latter studies failed to control for plaque.

Gender-related psychosocial factors may partially explain differences in risk for AP between males and females. Social norms are believed to influence the male tendency for increased risk-taking, for example, smoking; having less health-related knowledge and generally taking fewer preventive actions (Coburn and Pope 1974; Blaikie 1979; Locker 1989). All of these factors have been associated with an increased risk for periodontitis.

Gender socialization is also believed to influence social norms and health behavior (Nathanson 1977). Women may be socialized to feel more susceptible to disease, be more sensitive to symptoms and to seek more frequent health care. Social norms may therefore help to account for males seeking less regular dental care, being less well informed about disease prevention and having more untreated disease.

Gender, like age is a determinant of AP and its inclusion in modeling necessary to control for its confounding influence on AP. In the current study, all three models contained gender. Gender was not significant in any of the models.

Model \#1 showed a slightly higher risk within the total study population of 1.37 times when males were compared to females although the difference was not significant, $\mathrm{p}=0.23$.

Model \#2a showed an increased risk for males when compared to females within those aged 35 - 54 years although the difference was not significant, $\mathrm{OR}=1.22, \mathrm{p}=0.55$.

Model \#2b showed more of a trend for increased risk for males compared to females within the older sub-population, $\mathrm{OR}=1.78, \mathrm{p}=0.13$. 


\subsubsection{Demographic Variables Not Found in the Models}

It was not the purpose of the current study to consider the influence on AP of other demographic variables such as population density, rural versus urban setting, or dentist to population ratio. The primary reason that the influence of these variables on periodontitis was not studied was that most study subjects live in Winnipeg, were attending the dental clinic rather than private dentists and would have their visiting behavior influenced by student and clinic administration.

\subsubsection{Socioeconomic Status Variables}

Societies are generally divided into hierarchical social strata (Beal 1989). Socioeconomic status (SES) is a standard index which assesses education, income, and occupation. The influence of each of these variables on a history of periodontitis was investigated in the current study. Other psychosocial variables were also considered including employment status, marital status and life-stress.

\subsubsection{Marital Status}

In the current study, univariate analysis showed that being single had an unadjusted $\mathrm{OR}=1.71, \mathrm{CI}=1.07-2.72$, that is, those who were single had an increased risk of 1.71 times for a history of AP compared to those with partners. Once age-gender-adjusted, the $\mathrm{OR}$ increased, $\mathrm{OR}=1.81, \mathrm{CI}=$ $1.10-2.97$, indicating that the differences in AP due to marital status were not due to the confounding influence of gender or age.

The current study also showed that having a partner was correlated with having an annual family income $\geq \$ 25,000$, likely due to the partner's additional income. There was no evidence that those with partners were younger or more likely to be employed which could have explained their higher family income. 
Although many studies have shown that with increased social support there is a lower prevalence of morbidity/mortality, the mechanism by which social support influences health status is unclear (Berkman and Syme 1979). Marital status is not believed to influence the prevalence/ severity of AP directly. Being single has been shown to have an indirect association with AP due to its association with lower levels of income and education and related poorer oral hygiene, infrequent dental visiting and increased health compromising behaviors (Bullough 1972; Calnan 1985). Social isolation, lack of social network, alienation and powerlessness have been shown to be associated with poor preventive behavior (Bullough 1972). Single individuals may lack reinforcement from a social network so that positive health behaviors are not reinforced or sustained (Blaikie 1979; Berkman and Syme 1979).

There is some evidence for spousal influence on dental behavior (Dielman et al 1982). Within 273 New Jersey families, a high degree of companionship between a couple was shown to be related to positive health care practices and use of preventive health services. The cohesiveness within a family has been shown to be related to physical activity, nutrition and positive health behavior for the family members (Pratt 1972).

Some studies have not shown a relationship between preventive behavior and the proportion of friends or family in an individual's social network (Salloway and Dillon 1973). Inconsistencies in the literature regarding the influence of others on health status or preventive practices may be due to difficulty in measuring and studying social/familial factors and differences in populations studied.

Model \#1 retained marital status subsequent to logistic regression analyses however income was not present. Marital status may have 
accounted for some of the differences between cases and controls regarding income since being single was correlated with having a lower income.

Model \#2a failed to show a significant association with any socioeconomic variables through univariate analysis therefore none was considered in the model building process. There may also have been too little variation in level of education within young adults making it difficult to determine the effect of education on AP.

Model \#2b included marital status. Being single increased risk within the older adults by $\mathrm{OR}=3.07$ times, $\mathrm{p}<0.008$.

The differences observed among the three models regarding marital status may be explained by considering the three different age groups investigated. Being single increased risk among the total study population, mainly for those $\geq 55$ years but not for the younger adults. Having a partner generally represents social/family support. In the current study, single young adults may have perceived family support from their parents and social support from their working colleagues while many of the $\geq 55$ year olds would not have had surviving parents and those who were retired would presumably have had a smaller social network, leaving single elderly individuals without as strong a support system. Lack of such a support among the elderly may influence their preventive behaviors awareness of health services or access to dental care.

\subsubsection{Income}

There was not an increased risk for a history of AP associated with level of income among the total study population or among the young adults in univariate analysis. However, among the older adults, there was a trend for those with $\geq \$ 25,000$ annual income to be at increased risk for AP. An 
association which remained meaningful even after controlling for age and gender.

\section{MODEL \#1:}

Income was not found to be significant in Model \#1 because age explained much of the variation between cases and controls that is, increased age was correlated with lower income. This would be partially explained by the fact that more of the older individuals were retired or homemakers. Marital status may have accounted for some of the differences regarding income since being single was correlated with having a lower income.

\section{MODEL \#2a:}

Income was not a significant variable in the young adult model. Since all of the young adults were below the age of retirement and few were unemployed, it may be that there was not enough variation in their income to find an effect of income on risk for AP.

\section{MODEL \#2b:}

Among the older adults, there was a trend of having an annual family income $<\$ 25,000$ decrease the risk for a history of periodontitis by OR $=0.52, \mathrm{p}=0.09$. Although previous research has shown increased prevalence of periodontitis among those with low income (Miller et al 1987; Brown et al 1990; Locker and Leake 1993), the current study showed that annual family income $<\$ 25,000$ was not associated with increased risk among any of the age groups. In fact, among those $\geq 55$ years, there was a trend for decreased risk of AP by $50 \%$ for those with an income $<\$ 25,000$. This finding is most likely related to the observation that income $\geq \$ 25,000$ was correlated with having $\geq 25$ teeth present. Authors have reported that 
the presence of more teeth may allow for a greater chance of diagnosing a history of periodontitis and other dental diseases since involved teeth have not been extracted (Beck 1984; Page 1984; Hunt et al 1985; Beal 1989). The current study showed a correlation between having $\geq 25$ teeth and a higher family income.

Education may have been represented by proxy in the model by income since higher education was correlated with higher income. Both higher income and education were correlated with having $\geq 25$ teeth. This finding could help explain why those with higher income appeared to be at increased risk for AP. The older individuals with a higher income had more teeth present in which to diagnose AP than those with lower income.

It should be remembered that it was not the individual's income that was investigated in the present study, but rather their past year's annual family income. Had a long-term history of the individual's income been available, it is possible that a clearer relationship would have been observed between periodontal status and income. Long-term exposure to low income would be more indicative of the influence of income on a history of AP than one year's income.

Among the study population, $46.8 \%$ had an annual income $<\$ 25,000$ while the same income among Manitobans (rural and urban) is $21.1 \%$, suggesting that participants in the study were low income earners (Statistics Canada 1993).

\subsubsection{Socioeconomic Status Variables not found in the Models.}

Occupation did not appear in any of the final models although in univariate analysis there had been a trend within the total study population and young adults for those with professional/managerial jobs to be at lower risk for a history of periodontitis. 
The current study failed to show an effect of life-stress on a history of AP for any age-group. The method of measuring life-stress may have been inadequate to reveal its influence, if any. Many respondents seemed to struggle with assessing life-time experience with stress. Most experienced periods of extreme stress as well as periods of no stress. As a result, there was a tendency for many respondents to report "average" life-stress.

Additional psychosocial differences between cases and controls including life-stress may have been observed in the current study had edentulous individuals not been excluded and their periodontal histories available. It is speculated that the edentulous have lost many of their teeth to AP. The inability to include these individuals in the current study may have underestimated the influence of psychosocial variables on a history of periodontitis.

There are other difficulties in assessing the influence of psychosocial variables on chronic diseases. These difficulties have been related to unreliable and subjective measurements of the variables and to the complex interaction among the determinants of health.

\subsubsection{Health Compromising Behavior}

Health behavior may promote, enhance and preserve good health, or if the behavior is harmful, such as smoking, may compromise health (Last 1988). The current study investigated whether any of the following behaviors were associated with a history of AP: frequency of toothbrushing, flossing, dental visiting, and cigarette smoking.

Previous studies have reported a lower prevalence/ severity of AP associated with regular of dental visiting (Nikias et al 1975; Chen and Stone 1983), toothbrushing and flossing (Nikias et al 1975; Brown et al 1990; Syrajala et al 1992) while cigarette smoking has consistently been 
associated with an increase in AP (Levy et al 1987; Beck et al 1990, 1992; Locker and Leake 1993).

\subsubsection{Cigarette Smoking}

In univariate analysis, the current study showed an un-adjusted OR $=1.64, \mathrm{CI}=1.07-2.51$, for ever-smokers compared to non-smokers within the total study population. Once age-gender adjusted, the $\mathrm{OR}=1.82, \mathrm{CI}=$ $1.13-2.92$, indicating that differences between cases and controls regarding their exposure to smoking was not due to differences in age or gender. Being male was correlated with a history of smoking. The latter finding has also been reported by others (Borlund and Rudolph 1975) and may be due to greater risk-taking behavior among males or to participation in more health compromising behaviors. Being male was also correlated in the current study with less regular toothbrushing and dental visiting and higher plaque scores, all previously associated with increased risk for AP.

In univariate analysis among younger adults, the unadjusted OR = $1.88, \mathrm{CI}=1.02-3.78$, was similar to the risk experienced by the total study population after age-gender adjusting, showing once more that the effect of smoking was not explained by age or gender. Among those aged $\geq 55$ years, the unadjusted $\mathrm{OR}=1.87, \mathrm{CI}=0.93-3.78$, showing a trend for significance. Both age-groups were further subjected to age-gender adjustment through the modeling process. Subsequently, those $\geq 55$ years failed to show an effect of ever smoking.

For the purposes of the current study, a history of ever smoking cigarettes was converted into a variable which attempted to consider lifetime exposure to cigarette smoking. A cigarette index was calculated by multiplying number of cigarettes/day $\mathrm{x}$ number of years smoked. The total life-time-exposure to cigarettes could be further calculated by multiplying 
the cigarette index x 365 days. A dose-response effect was significant for the cigarette index prior and subsequent to age-sex adjustment for the total study population and for the younger adults, but not for those $\geq 55$ years.

Model \#1 showed an increased dose-response OR for the cigarette index: $1-300=\mathrm{OR}=1.24 ; 301-500=\mathrm{OR}=1.78 ;>500=\mathrm{OR}=3.80$ compared to non-smokers. Indirect evidence of a dose-response gradient in smoking exposure has been reported previously (Bergstrom et al 1991).

Model \#2a showed even a higher magnitude of effect for doseresponse for the cigarette index among the younger adults: 1-300 cigarette years $=\mathrm{OR}=1.00 ; 301-500=\mathrm{OR}=3.15 ;>500=\mathrm{OR}=7.33$ when compared to non-smokers. There was a greater magnitude of effect within the young adults once those $\geq 55$ years had been removed from analysis. This finding was probably due to the differential in the number of teeth present between the two groups.

Model \#2b did not include cigarette smoking behavior. One explanation for the absence of cigarette smoking in the older adult model may be that fewer individuals with a high life-time smoking exposure survived over 55 years of age. Another explanation could be that those who have survived may represent individuals who, genetically, may be more resistant to chronic diseases. It may also be explained by the exclusion of edentulous individuals from the present study, many of whom were likely smokers and lost their teeth to periodontitis. There is a higher proportion of edentulism among smokers than nonsmokers (Johnson et al 1988). Exclusion of either group could have resulted in an under-estimation of the effect of smoking on periodontal health.

The findings in Model \#1 (total population) and \#2a (younger adults) are consistent with the literature. Cross-sectional studies which measured current/ever smoking and AP concurrently, have shown an increased 
prevalence/severity of AP to those exposed to smoking (Levy et al 1987; Beck et al 1990; Locker and Leake 1993). The magnitude of the association of AP and smoking varies among studies. Increased $\mathrm{OR}=2.9$ and $\mathrm{OR}=6.2$ were reported among Black and White elderly North Carolina smokers respectively when compared to non-smokers. Two previous case-control studies reported smokers to have an $\mathrm{OR}=2.5$ (Bergstrom 1989) and $\mathrm{OR}=2.6$ (Haber and Kent 1992) compared to nonsmokers when age and sex-adjusted and plaque was controlled. Differences in populations studied, designs, and diagnostic cut offs of AP may explain differences in ORs among studies.

\subsubsection{Variables Not in the Models.}

A number of behavioral variables which were significant in previous studies failed to be associated with increased risk in the current study, including dental visiting, toothbrushing and flossing.

\section{DENTAL VISITING:}

In univariate analysis, there was a decreased risk of a history of AP among the total study population for those who reported to have regular dental visits (an unadjusted $\mathrm{OR}=0.62, \mathrm{CI}=0.40-0.97$ ). However, once agegender adjusted, there remained only a trend for this protective influence indicating that dental visiting was influenced by age and gender. Older age is correlated with having $<25$ teeth. Previous studies have shown that regularity of dental visiting is more likely among those who have teeth present (Locker and Leake 1993).

Regularity of dental visiting was not present in any of the age group models. This may be due to the strong influence of age and the number of remaining teeth in Models \#1 and \#2b. Dental visiting may also have been represented in the models by gender since being male was correlated with 
less regular dental visiting. A pattern consistent with previous studies (Hayward et al 1994).

Dental visiting has been reported to be less frequent among the elderly (50 - 87 year old Ontario residents) than other age groups, but it is rising among the new elderly (Payne and Locker 1992). The overall utilization rate by the elderly is low due to the low rate of attendance by those who are edentulous when compared to the dentate elderly (Haefner et al 1967; Payne and Locker 1992). This is consistent with the results of the current study which showed a correlation between having $\geq 25$ teeth and increased regularity of dental visiting. The reasons given by the elderly for non-attendance are: "nothing wrong", or "can't afford it". Non-attendance has also been associated with being edentulous, not having dental insurance, and $<\$ 20,000$ annual income (Locker et al 1991).

Dental visiting has previously been associated with low SES (Haefner et al 1967; Antonovsky and Kats 1970). The current study showed that having > Grade 12 education was correlated with more regular dental visiting. Income was not correlated with dental visiting perhaps because patients at the Faculty of Dentistry experience less of a financial deterrent as a result of reduced fees.

In the current study, frequency of dental visiting was not assessed in the telephone interview but rather through the questionnaire from existing FOD records. The reliability and validity of the question, "Do you have regular dental appointments?" was not evaluated. The term 'regularity' was not operationally defined. However, this flaw would not have resulted in a systematic bias since the question was equally undefined for both cases and controls. The poorly designed question may have resulted in an underestimate of the influence of regularity of dental visiting on a history of periodontitis. 


\section{TOOTHBRUSHING:}

The current study did not find an increased risk for a history of AP with a reported toothbrushing $<1 /$ day. There was no difference between the cases and controls regarding frequency of toothbrushing. These findings may be due to the fact that $90.3 \%$ of the total study population reported to brush $\geq 1$ /day and there were very few controls $(n=15)$ who failed to brush at least once daily. Similar rates of toothbrushing have been reported in the United States (88\%) (Nikias et al 1975) and among older Ontario residents (Payne and Locker 1992). It is believed that the prevalence of daily toothbrushing is high due to many years of promoting the behavior.

The current study, as well as previous studies must recognize the potential influence of social acceptability bias in self-reported behaviors such as toothbrushing. This bias is difficult to control. However, it was felt that the nature of the current study was able to diminish the bias because of its non-threatening, confidential telephone interview. If subjects did overreport their brushing frequency, it could have resulted in an underestimate of the influence of toothbrushing frequency on a history of AP.

In the current study, being male and having a higher plaque score were correlated with less regular toothbrushing, findings consistent with the literature. The former observation has been explained by differences in genders regarding disease prevention knowledge and behavior.

\section{FLOSSING:}

The reported frequency of daily flossing among the study population was $24.3 \%$, somewhat higher than that $(15.9 \%)$ reported in a survey of randomly selected adults in the United States (Harris and Guten 1979). Frequency of flossing for interproximal cleansing of the teeth was found not 
to be related to a history of AP in the current study. Flossing frequency was not correlated with toothbrushing or level of plaque score. The former may be due to over-reports of toothbrushing and the latter to inadequate flossing technique among some who report daily flossing.

Studies of elderly adults have shown that use of interproximal cleaning adjuncts is uncommon and infrequent (Payne and Locker 1992). The infrequent use of floss compared to toothbrushing may be related to its more recent introduction into the daily home-care regimen. In addition, flossing is a more technically challenging and less comfortable procedure. Failure to floss may also be associated with less social disapproval than failure to brush.

The influence of SES on health behavior must be recognized. The current study found increased education was correlated with increased toothbrushing and dental visiting. Increased levels of education and income have been shown to be correlated with increased dental visiting in other studies as well (Nikias et al 1975; Blaikie 1979). These associations may exist because those with higher education perceive fewer barriers to preventive behavior (Kasl and Cobb 1966; Syrajala et al 1992). Behavioral differences may also be related to variations in access to health knowledge, beliefs in susceptibility (Williams 1972) or differing relative values regarding desired health outcome (Blaikie 1979; Rundall and Wheeler 1979) or a sense of hopelessness, fatalism and having an external locus of control among those of lower SES (Norman 1986). Differences may also be related to the dental care delivery system's attitude toward lower SES groups (Blaikie 1979). Some studies show that even when financial barriers are eliminated, preventive dental visiting is less frequent among the lower SES (Manning et al 1986). 
Traditional behavioral models such as the Health Belief Model (Rosenstock 1966; Norman 1986) and Fishbein's Theory of Reasoned Action (Norman 1986; Glanz et al 1991) have attempted to explain and predict health behavior but have failed to account consistently for variations in behavior among SES groups (Rundall and Wheeler 1979; Chassin et al 1984). Despite these challenges, an attempt at understanding how psychosocial variables influence health and health behavior seems essential in the design, implementation and evaluation of prevention or health promotion activities.

\subsubsection{Chronic Conditions/ Diseases}

The influence of a number of chronic conditions and diseases on a history of AP was investigated in the current study. It was believed that AP, like other chronic conditions may be influenced by or associated with these conditions and diseases. The diseases which were investigated included: diabetes and ischemic heart diseases (angina, myocardial infarction and arterio/atherosclerosis). Hypertension was also investigated.

\section{DIABETES:}

There were 16 subjects who had been diagnosed with diabetes and an additional six who had been told they had borderline diabetes. Combined, this constituted a $5.8 \%$ prevalence of diabetes among the total study population $(\mathrm{n}=382)$. This corresponds to the $6 \%$ prevalence of diagnosed diabetes/current fasting plasma glucose of $\geq 7.8 \mathrm{mmol} / \mathrm{L}$ among adults 18 years and older in Manitoba in 1991 (Young et al 1991). Although the current study population is not meant to be representative of all Manitobans, consistency of disease prevalence data between the two populations supports the internal validity of the measures used by the current study to determine disease exposure. 
No attempt was made to distinguish insulin-dependent diabetes mellitus from non-insulin dependent diabetes mellitus despite the fact that age-of-onset was available. The prevalence of the singular conditions was too low to analyze.

In univariate analysis, there was a trend for those with a history of diabetes to be at increased risk of a history of AP with an un-adjusted $\mathrm{OR}=$ $2.32, \mathrm{CI}=1.25-6.69$. However, when age-gender adjusted, the risk was no longer apparent. These findings may be explained by the influence of age on the prevalence of diabetes. Increased age was correlated with diagnosed diabetes in the current study and diabetes has been shown previously to be associated with increased age (Young et al 1991).

The literature contains conflicting reports regarding an association between diabetes (IDDM and NIDDM) and a history of AP. While some studies show an increased OR of approximately three times (Emrich et al 1993), others fail to show an association when confounding variables were controlled (Barnett et al 1984). This lack of consistency may be due to differences in populations studied and methods of measuring both AP and diabetic status.

\section{ISCHEMIC HEART DISEASE:}

Among the total study population $(\mathrm{n}=382)$ only 12 subjects reported a history of Myocardial Infarction, 27 Angina Pectoris, and 21 Arterio/ Atherosclerosis. Combined, 40 (10.5\%) of the study population had a history of one or more, (i.e., any) ischemic heart disease(s). Reported histories were not confirmed through medical consultation therefore the validity of the method of exposure-determination is uncertain. However, these diseases are generally perceived by patients as salient and serious such that they would not likely under-report their exposure. 
Within the study population $(\mathrm{n}=362$ ), univariate analysis revealed an increased un-adjusted OR for AP among those with a history of any ischemic heart disease, $\mathrm{OR}=2.20, \mathrm{CI}=0.98-4.94$. However, following agegender adjustment, there was no longer an increased risk for AP due to the strong influence of age on ischemic heart disease evidenced by the correlation between increased age and a history of any ischemic heart disease in the current study. Increased age has been associated with higher prevalence of ischemic heart disease in previous studies (Young et al 1991). Among the young adults, there were too few subjects reporting a history of any ischemic heart disease to complete analysis. No difference was observed between cases and controls regarding ischemic heart disease within the older adult population aged $\geq 55$ years.

\section{HYPERTENSION:}

Within the total study population $(\mathrm{n}=382), 96(25.1 \%)$ reported having been told by their physician that they had hypertension or high blood pressure. This percentage is somewhat higher than that (17\%) found within a random selection of adults aged 18 and over in Manitoba in 1991 (Young et al 1991). The prevalence may be higher in the study population due to differences in methods of determining hypertension and to the older ages of the current study population ( $35-87$ years) compared to $18+$ years in the Manitoba Heart Health Survey. In the current study, when previously diagnosed hypertension was combined with clinical measurements of SBP $>140$ or $\mathrm{DBP}>90 \mathrm{~mm} / \mathrm{Hg}, 131(34.3 \%)$ had a history of hypertension suggesting that not all subjects with elevated blood pressures at the time of their clinical periodontal examination had been diagnosed by their physician as having high blood pressure. These additional case findings 
support the continued evaluation of blood pressure for patients attending the FOD dental clinic.

In univariate analysis within the study population $(\mathrm{n}=362)$, diagnosed hypertension alone, was associated with an increased unadjusted $\mathrm{OR}=1.91, \mathrm{CI}=1.14-3.19$, while the combined variable was associated with $\mathrm{OR}=1.82, \mathrm{CI}=1.14-2.89$. Neither remained significant when age-gender adjusted, indicating the influence of age on hypertension, evidenced by the fact that the current study showed increased diagnosed hypertension was correlated with increased age, as has been reported elsewhere (Young et al 1991).

None of the chronic diseases or conditions investigated were associated with an increased risk for AP when age-gender adjusted. This finding may be due to one or more of the following: 1) there is no true association; 2) the prevalence of certain conditions may have been inadequate to identify differences, Type II error; 3 ) the chronic conditions were under-control through medication, thus diminishing their potential effect on AP; 4) the chronic condition may have been undiagnosed. The last three scenarios would have resulted in an under-estimation of the influence of these conditions on AP. Further study is warranted.

\subsubsection{Use of Medications With Potential Drug Actions}

The influence of a number of medications on a history of AP were investigated including: dilantin, cyclosporin, nifedipine and estrogen/progesterone.

The reported use of the medications of interest was low among the study population ( $\mathrm{n}=382$ ), even when analysis considered use of 'one or more' of the first three medications: Dilantin $=2$, Cyclosporin $=0$, Nifedipine $=3$. Estrogen/Progesterone had been used by $143(64.1 \%)$ females . 
Inadequate exposure to the medications curtailed further study of all but estrogen/progesterone. The unadjusted OR for estrogen/progesterone showed a trend for reduced risk among those who had ever taken the medication, $\mathrm{OR}=0.62, \mathrm{CI}=0.35-1.12$. However, subsequent to age adjustment, there was no longer an association, suggesting that more of the young women may have reported ever having taken the medication. This finding could be related to more frequent use of oral contraceptives among women aged 35 - 54 years or to recall bias which could have diminished the older women's recollection due to their more distant use of the medication. There was no evidence of increased risk for AP or BOP (gingivitis) in the current study although the latter has been reported previously (Knight and Wade 1973). BOP associated with use of hormones is likely transient. Increased BOP may not have been evident in the current study because of the cross-sectional assessment of BOP and inability to determine when the subjects were exposed to the hormones.

\subsubsection{Oral Findings}

\subsubsection{Plaque Score (PS)}

Within the total study population $(\mathrm{n}=382$ ) there was a fairly even distribution of plaque scores: Approximately $50 \%$ had a PS $<50 \%$. Only onethird of the population had a PS $\geq 67 \%$. Regardless of how one assessed PS, univariate analyses showed an increased risk for AP with increased plaque, unadjusted $\mathrm{OR}=1.84(\geq 50 \%)$ and $\mathrm{OR}=2.59(\geq 67 \%)$. The $\mathrm{OR}$ decreased somewhat but remained significant subsequent to age-gender adjustment indicating that some of the difference was explained by age and gender.

Plaque $(\geq 50 \%)$ was entered into Model \#1 for the total study population. The unique effect of plaque on a history of AP was not 
significant, but showed a trend with an increase $O R=1.55, p=0.08$. Plaque may have shown a greater effect but age and gender were present to explain much of the variation in this model. Other studies have shown that the elderly (Haefner et al 1967; Payne and Locker 1992) and males (Kasl and Cobb 1966; Williams and Wechsler 1972; Wilson and Elinson 1981) generally have a higher plaque level.

It must be remembered that the PS was taken only once for the current study. Plaque scores are known to be transient and thus would be limited in their association with increased risk for lifetime experience with AP.

\section{BLEEDING ON PROBING (BOP):}

Within the total study population $(\mathrm{n}=382)$, few had $\geq 67 \%$ BOP $(8.1 \%)$ while over half had $0-33 \%$ BOP $(57.6 \%)$ and the remainder had moderate BOP $34-66 \%$ ( $34.3 \%)$. Since so few had $\geq 67 \%$ BOP, cell size was inadequate, and $\geq 50 \%$ BOP $(24.7 \%)$ versus $<50 \%(75.3 \%)$ was used for analyses.

Within the study population $(\mathrm{n}=362)$, univariate analysis showed an increased risk for $\mathrm{AP}$ among those with $\geq 50 \% \mathrm{BOP}, \mathrm{OR}=2.47, \mathrm{CI}=1.41$ 4.30. Subsequent to age-gender adjustment, the OR remained significant, $\mathrm{OR}=2.40, \mathrm{CI}=1.33-4.32$ indicating that age-gender did not explain the difference in BOP between cases and controls.

Among the young adults, the $\mathrm{OR}=2.67, \mathrm{CI}=1.26-5.6$, remained significant while older adults were not at increased risk for AP when they had $\geq 50 \%$ BOP, OR $=2.17, \mathrm{CI}=0.87-5.43$. Differences between the two stratified age groups regarding their risk to $\mathrm{AP}$ due to $\mathrm{BOP} \geq 50 \%$ may be related to the differential number of teeth present. The elderly group had fewer teeth present to be susceptible to disease or on which to detect BOP. 
BOP was not correlated with plaque score in the current study. This finding may be explained by the plaque removal capability of subjects just prior to the cross-sectional dental exam. BOP scores provide evidence of long-term plaque removing behavior, the latter being unavailable in the current study.

\subsubsection{Number Of Teeth Present}

Preliminary to final model building, the influence of the number of teeth present was investigated. It was determined that inclusion of $<25$ teeth would mask the effects of other potential explanatory variables. It was decided to eliminate this risk marker from further analysis.

Having $<25$ teeth present was correlated with $\leq$ Grade 12 education, $<\$ 25,000$ annual income, and irregular dental visiting. These findings were related to age that is, the elderly had fewer teeth present and they were also more likely to have lower income and education than the younger adults.

In the total study population, controls had a significantly higher mean number of teeth present $(24.05, \mathrm{SE}=0.42)$ than cases $(22.33, \mathrm{SE}=0.33)$, $\mathrm{t}=3.3, \mathrm{p}<0.001$. Similar tooth loss patterns have been reported for other populations. Among United States employed aged 18 - 64 years, the mean number of teeth present in 1987 was 24; and among United States seniors aged $\geq 65$ years, 17 teeth were present while $41 \%$ of the older group were edentulous. The current study's elderly were $\geq 55$ years, not as old as the United States seniors and the current study only included dentulous individuals, while the United States study averaged the number of teeth present, including the edentulous. Both factors would explain the higher number of teeth present in the current elderly population (Miller et al 1987). Among Iowans aged $>65$ years, the mean number of teeth was 19 (Beck et 
al 1987b). Among dental school patients in Sweden, 25-year olds had a mean of 26.8 while those 75 years old had 14.6 teeth (Papapanou et al 1988). Among patients $\geq 65$ years of age attending the University of Saskatchewan Dental School, $41 \%$ were edentulous at the time of examination or were treatment planned to be so and an additional $61 \%$ were partially edentulous (Hoover and McDermott 1990). The prevalence of edentulism among the elderly attending the FOD, University of Manitoba is uncertain. However, the latter study and the study of United States seniors suggest that the prevalence of edentulism among those $\geq 65$ may be approximately $40 \%$.

In the present study, there was a higher proportion $(66.9 \%)$ of subjects having $\geq 25$ teeth present among the controls compared to the cases, $x^{2}=27.64,1 \mathrm{df}, \mathrm{p}<0.0001$. This proportion was even greater $(84.3 \%)$ when assessed among the young adults alone, $\mathrm{x}^{2}=17.27,1 \mathrm{df}, \mathrm{p}<0.0001$. However, there was no significant difference between cases and controls regarding $<25$ teeth within those aged $\geq 55$ years, $\mathrm{x}^{2}=0.12,1 \mathrm{df}, \mathrm{p}=0.73$. Having fewer than 25 teeth present among the young adults is likely a marker of previous periodontal disease. This finding is consistent with other studies which have shown that those with fewer teeth present are at increased risk for AP (Papapanou et al 1988; Locker and Leake 1993). It is possible that the older adults in the current study would have been influenced similarly had they presented with more teeth. The fact that the older adults were not at increased risk for a history of AP when they had < 25 teeth could be explained by the study's inability to identify a history of AP for the teeth which had been lost prior to the periodontal exam.

\subsection{CONCLUSIONS}

The current study attempted to further the understanding of chronic adult periodontitis among patients attending the dental teaching-clinic, 
Faculty of Dentistry, University of Manitoba. It did so by analyzing the influence on AP of a number of variables known to be associated with other chronic diseases/conditions. In addition to demographic determinants and dental disease markers, analyses included the influence on AP of socioeconomic variables, exposure to chronic diseases/conditions, use of several specific medications and health compromising behaviors.

Due to the design of the current study, diagnosis of a history of AP was based only on teeth which were present at the time of the periodontal examination, disregarding the fact that some individuals had previously lost some of their teeth to AP. This study limitation would have resulted in mislabeling some of these individuals as controls, thus underestimating the influence of the explanatory variables on AP. Other design limitations must be considered, particularly the potential for recall and social desirability bias. The results of the study should not be generalized beyond the target population and, due to the study's inclusion/exclusion criteria, may not be applied to all patients who seek care at the FOD.

The current study contributes to our understanding of adult periodontitis in several ways. It confirms the results of previous studies indicating increased risk associated with cigarette smoking. A clear doseresponse effect of smoking was observed, particularly among young adults. In addition, the study identified that being single was associated with increased risk, an association observed with other chronic diseases but not widely noted in the dental / periodontal literature. The epidemiological methods employed in the study could provide a model for future studies in periodontology and other chronic dental conditions. 
1. Univariate analysis indicated that being $\geq 55$ years or older was associated with $4 \mathrm{x}$ increased risk for a history of AP. Multivariate analysis showed a $1.06 \mathrm{x}$ increased risk with each year among the total study population.

2. A dose-response effect was observed with the cigarette-index. There was a $7.3 \mathrm{x}$ increased risk among the 35 to 54 year old heaviest smokers and $3.8 \mathrm{x}$ among the total study population. Those aged $\geq 55$ years had no apparent risk associated with smoking.

3. Being single increased risk in the total study population by $1.77 \mathrm{x}$ and $3.07 x$ among those $\geq 55$ years. Single $35-54$ year olds were not at increased risk compared to those with partners.

4. Low income was not associated with increased risk.

5. There was a trend for $1.55 \mathrm{x}$ increased risk associated with $\geq 50 \%$ plaque. Multivariate analysis indicated no apparent increased risk for AP associated with gender, dental visiting, brushing / flossing, chronic conditions/ medications with potential drug action or life stress.

\subsection{RECOMMENDATIONS}

1. Based on the results of the current study and on the growing scientific evidence that cigarette smoking increases the risk of chronic adult periodontitis, further study is warranted.

2. Future studies should be longitudinal and determine the effect on AP of:

a) community intervention programs of smoking cessation

b) decreased interval of supportive maintenance for smokers with AP 
3. As the current study showed an increased risk for AP among single adults, $\geq 55$ years, the mechanism of action on AP should be investigated.

4. Although the current study did not detect an increased risk of AP due to chronic diseases / conditions or to use of study medications, further study is warranted:
a) within a population where prevalence of exposure is higher than in the current study
b) using a longitudinal cohort study design

\subsection{IMPLICATIONS}

The objectives of the current study did not include issues of planning, implementation or evaluation of preventive programs at the Faculty of Dentistry. However, health promotion activities aimed at reducing risks identified through the current study are discussed. Potential implications and the results, along with the results of the current study, will be reported to the Dean of the FOD. The administration of the FOD may wish to consider strategies to supplement existing preventive programs. In addition, a summary of the results will also be sent to the study participants who had requested feedback.

A realistic goal of periodontal disease prevention set by the WHO for the year 2000 is, "the retention throughout life of a functional, esthetic, natural dentition of not less than 20 teeth and not requiring a prosthesis" (WHO 1982). This goal should be considered when planning programs of prevention at the FOD. For example, it may be unrealistic, in fact unnecessary, to attempt to prevent gingivitis or slight adult periodontitis among everyone since previous epidemiological studies have shown that all individuals are not at equal risk for moderate-severe periodontal 
destruction (Löe et al 1978, 1986). The current study suggests that within the dental teaching-clinic population, there is evidence of increased risk for a history of AP among certain sub-populations. The effects of directing preventive programs at these groups should be investigated further.

The current study showed that the risk of a history of chronic adult periodontitis (AP) among the dental teaching-clinic population was influenced by several variables (Table 9.1). Within the total population (35 87 years), increased age, a higher cigarette index, a greater plaque score and being single were shown to increase risk for a history of AP. When age stratified, younger adults ( 35 - 54 years) had an increased risk for AP associated only with a greater cigarette index, while older adults $(\geq 55$ years) who were single or who had a family income $\geq \$ 25,000 /$ year were at increased risk. Strategies of health promotion which would permit the Faculty of Dentistry to address these variables are discussed.

\section{LEVELS OF PREVENTION:}

In general, prevention of periodontal disease has been broadly based, involving primary, secondary and tertiary prevention.

Primary prevention involves the control of causative factors of disease by reducing risk factors, environmental exposures, and/or providing early interventions (Frazier and Horowitz 1990; United States Department of Health and Human Services 1992). Primary periodontal disease prevention attempts to deter significant clinical attachment loss in healthy sites/individuals (Imrey 1986) but does not imply complete control of gingivitis (Frandson 1984). Fluoridation is an effective means of primary prevention of dental caries in communities (WHO 1970; Graves et al 1986) however, a similar agent has not been identified for the prevention of AP. Therefore, personal oral hygiene at the individual level is most often 
advocated as a means of primary prevention (Heifetz and Suomi 1973; Burt et al 1984).

Secondary prevention attempts to deter clinical attachment loss among sites/individuals who have some probing depth or pre-existing attachment loss (Imrey 1986; Frazier and Horowitz 1990) through early case finding and treatment (United States Department of Health and Human Services 1992). Early treatment has been shown to improve prognosis (Frandson 1984). Periodontal treatment generally consists of scaling and root planing (i.e., root surface debridement) and sometimes periodontal surgery.

Tertiary prevention employs reconstructive measures to immobilize teeth and prevent tooth loss in sites/individuals with advanced attachment loss (Imrey 1986). This may be accomplished by providing supportive and rehabilitative services thus minimizing further destruction and maximizing the individual's quality of life (United States Department of Health and Human Services 1992). Edentulous areas may be reconstructed with prosthetic devices such as bridges, partial/full dentures or through the use of dental implants. Additional periodontal destruction is generally controlled through periodic periodontal supportive recall appointments.

\section{Existing Program of Periodontal Prevention At FOD:}

All three of the aforementioned levels of prevention are currently incorporated into the undergraduate periodontics program at the FOD as evidenced by the following brief program description.

\section{Case Finding:}

Patients receive a general oral screening at the FOD followed by dental and periodontal examinations. Diagnosis, prognosis and treatment plans are formulated based on the case findings. 


\section{Patient Education and Oral Hygiene Instructions:}

Patient education at the FOD attempts to increase the patient's awareness, knowledge and understanding of periodontal disease and its etiology. Based on the scientific literature, personal toothbrushing and flossing are recommended along with adjunctive aids to control/prevent AP (Löe et al 1965; Heifetz and Suomi 1973; Lindhe et al 1973; Abdellatif and Burt 1987). Oral hygiene instructions have been shown effective in reducing patient's plaque scores (Lightner et al 1971).

All patients who are accepted for treatment as new patients into the FOD and those returning for follow up care receive an oral hygiene assessment and individualized instructions in oral self-care.

\section{Scaling and Root Planing (SRP):}

The beneficial effects of subgingival plaque and calculus removal have been documented (Lovdal et al 1961; Philström et al 1981; Lang 1984). Root planing has been advocated to smooth root surfaces in order to discourage plaque accumulation; to eliminate endotoxin-contaminated cementum; and to better ensure complete calculus removal. SRP is provided, where appropriate, for all new periodontal patients at the FOD as part of their initial periodontal therapy and also for patients returning for supportive recall therapy.

\section{Periodontal Surgery:}

Periodontal surgery has been an integral part of periodontal therapy for decades. The efficacy and effectiveness of periodontal surgery have been established through longitudinal prospective studies, although many studies have been quasi-experimental and have not included analyses of 
cost-effectiveness or quality of tooth-years in comparison to non-surgical therapy (Shanley 1980; Lang 1984).

Periodontal surgery is provided for patients at the FOD in whom scaling and root planing and improved oral hygiene are unable to establish a periodontal environment compatible with health.

\section{Periodontal Supportive/Maintenance Therapy:}

Supportive periodontal recall/maintenance programs are designed to reinforce oral hygiene instructions, remove re-occurring calcified and noncalcified accretions and to monitor periodontal disease activity. Longitudinal studies have demonstrated the effectiveness of recall programs (Lightner et al 1971; Axelsson and Lindhe 1978, 1981; Ramfjord 1987) however, the ideal interval of recall is less certain. Periodontal patients at the FOD are generally recalled twice yearly, once between September - December and once between January - May.

\section{COMPREHENSIVE PROGRAM OF HEALTH PROMOTION AT THE FOD:}

Based on the results of the current study, cigarette smoking appears to increase the risk of AP among the dental teaching-clinic patient population. This finding is consistent with the results of numerous other studies in a variety of populations. However, few studies suggest incorporation of smoking cessation/prevention programs into programs of periodontal prevention (Haber and Kent 1992).

A number of "Individualist" Strategies directed at smoking prevention and/or cessation are outlined for considered by the FOD:

1. Dental health education at the FOD should include information for patients regarding the deleterious consequences of cigarette smoking on the periodontium. 
2. Smoking prevention programs should be incorporated into dental health education for adolescents since previous studies have shown that smoking is generally initiated during the adolescent years (Chassin et al 1984). This intervention would reinforce messages provided by public health programs and by other health professionals.

3. Linkages should be investigated between the FOD and The Manitoba Lung Association in order to establish a mechanism of referral for dental patients into programs of smoking cessation. The Lung Association operates a "Freedom from Smoking Clinic". The smoking cessation program is offered three times per year in the Fall, Winter and Spring. Each program consists of 8 sessions. Other agencies such as the Manitoba Cancer Society and the Heart and Stroke Foundation may have similar smoking cessation programs which could be investigated. The Lung Association has designed a number of age-specific smoking prevention/cessation pamphlets which could be made available for distribution at the FOD. Collaboration with the Lung Association in the preparation of additional health education materials could be investigated.

4. Patients who smoke and for whom periodontal surgery is planned should be advised of the consequences of smoking on periodontal health, in particular on healing following periodontal surgery (Preber and Bergstrom 1990) and such patients should be referred to programs of smoking cessation.

5. Additional studies should be carried out to identify individuals at increased risk for $\mathrm{AP}$ so that periodontal interventions may be more selectively provided. Until such time as there is sufficient evidence to formulate a true "High Risk Strategy", clinicians should include 
cigarette smoking along with the standard clinical parameters of probing depth, bleeding on probing, plaque score and clinical attachment loss to identify patients who may be at increased risk for AP (Grbic et al 1991).

6. Consideration should be given to reducing the interval of periodontal supportive recall appointments for smokers with periodontitis particularly since clinical signs of inflammation are often masked.

"Structuralist Strategies" should also be considered by the FOD as a means of smoking prevention/cessation. The structuralist or population strategy generally includes a community-wide approach of general health promotion as well as community participation in planning and implementation; community leader education; public dental health education; mass-media methods; environmental changes; and education of all health professionals to improve their health education programs and techniques (Sheiham 1991).

The FOD has been involved in numerous public dental health education programs over the years through the elementary/secondary school systems, community health fairs and through mass-media. The undergraduate dental curriculum provides students a basic understanding of the epidemiology of dental disease as well as disease prevention at the individual and community level. The Community Dentistry courses prepare students to participate in dental surveys and dental education programs. Based on the current study's findings regarding the influence of smoking on periodontal health, the FOD may wish to consider broadening its community and health promotion involvement.

7. The FOD should investigate becoming more involved in general disease prevention in the community, particularly with regard to 
smoking prevention and cessation. Involvement could be initiated by the FOD through the Manitoba Dental Association. For example, the Manitoba Medical Association has representatives on a healthactivist committee, Physicians for a Tobacco-Free Manitoba as well as on the national committee, Physicians for a Tobacco-Free Canada. Members of the FOD could provide scientific evidence to the MDA to support the establishment of a comparable committee within the MDA. Committee activities could include political actions to influence decision makers and governments (Frazier 1978) and/or to make regulatory changes in the social and physical environment (Locker 1989; Jeffrey 1989).

The FOD and MDA could work collectively with other health professionals to advocate social and political changes to enhance health. For example, health care providers could advocate at various levels of government to further control cigarette advertising. They could request additional tax sanctions to discourage the purchase of cigarettes or to impose and enforce sanctions on establishments which sell cigarettes to minors. Requests to the federal government could be made to identify and regulate nicotine as a drug. They could advocate economic incentives to farmers for producing an alternative crops to tobacco could be advocated (Jeffrey 1989).

\section{Marital Status:}

The results of the current study indicate that older adults ( $\geq 55$ years) who are single are at approximately three times greater risk for AP than those with partners. Although the exact influence of being without a partner on increased risk is not known, it is suspected that single elderly adults may lack support for: transportation to dental appointments and 
reinforcement of daily oral hygiene practices and personal grooming. Those who are single may not be discouraged from participating in healthcompromising behaviors as are those with partners.

8. The FOD should investigate the influence of marital status on AP. This may be done by determining whether programs directed at providing transportation for the elderly to the FOD or whether more frequent supportive periodontal appointments directed at reinforcing and encouraging oral self-care would improve their periodontal health. 


\section{REFERENCES}

1. ICD.9.CM (1979): International Classification of Diseases. 9th Revision. Clinical Modification. Vol 1, Tabular List. Jan 1979. CPHA, 1995.

2. Abbott RD, Yin Y, Reed DM, and Yano K (1986): Risk of Stroke in Male Cigarette Smokers. NEJM 315:717-720.

3. Abdellatif HM and Burt, BA (1987): An Epidemiological Investigation into the Relative Importance of Age and Oral Hygiene Status A: Determinants of Periodontitis. Journal of Dental Research 66:13-18 .

4. Abramson J. Survey Methods in Community Medicine, NY:Churchill Livingstone, 1990.

5. ADA (1987): News Briefs. ADA News April 6.

6. Aday L and Forthofer R (1992): A Profile of Black and Hispanic Subgroups' Access to Dental Care: Findings From The National Health Interview Survey. Journal of Public Health Dentistry 52:210-15.

7. Aeppli DM, Boen JR, and Bandt CL (1984): Measuring and Interpreting Increases in Probing Depth and Attachment Loss. Journal of Periodontology 56:262-64.

8. Africa CW, Parker JR, and Reddy J (1985): Bacteriological Studies of Subgingival Plaque in a Periodontitis-Resistant Population: I. Darkfield Microscopic Studies. J Periodont Res 20:1-7.

9. Ainamo J and Bay I (1975): Problems and Proposals for Recording Gingivitis and Plaque. Int Dent J 25:229-35.

10. Akesson L, Hakansson J, and Rohlin M (1992): Comparison of Panoramic and Intraoral Radiography and Pocket Probing for the Measurement of the Marginal Bone Level. J Clin Periodontol 19:326-32.

11. Anderson JA. Logistic Discrimination with Medical Applications. In: Discriminant Analysis and Applications, NY: Academic Press, 1973, p. 1-15.

12. Antonovsky A and Kats R (1970): The Model Dental Patient: An Empirical Study of Preventive Health Behavior. Social Science and Medicine 4:367-80.

13. Axelsson P and Lindhe J (1978): Effects of Controlled Oral Hygiene Procedures on Caries and Periodontal Disease in Adults. J Clin Periodontol 5:133-37.

14. Axelsson P and Lindhe J (1981): Effect of Controlled Oral Hygiene Procedures on Caries and Periodontal Disease in Adults. J Clin Periodontol 8:239-48.

15. Bader JD. Risk Assessment in Dentistry: Proceedings of a Conference, Chapel Hill: Univ NC, 1990. pp. 1-302.

16. Badersten A, Egelberg N and Egelberg J (1984): Reproducibility of Probing Attachment Level Measurements. J Clin Periodontol 11:475-485. 
17. Baelum V, Fejefskov O, and Karring T (1986): Oral Hygiene, Gingivitis and Periodontal Breakdown in Adult Tanzanians. J Periodont Res 21:221-232.

18. Bailit H and Manning W (1985): The Need and Demand for Periodontal Services: Findings from the RAND Health Insurance Experiment: American Academy of Periodontology Conference. Issues Related to the Delivery of Periodontal Care. AAP.

19. Barclay S, Thomason JM, Idle JR and Seymour RA (1992): The Incidence and Severity of Nifedipine-Induced Gingival Overgrowth. J Clin Periodontol 19:311314.

20. Barnett MI, Barker RL, Yancey JM, Macmillan DR and Kotoyan M, (1984): Absence of Periodontitis in a Population of Insulin-Dependent Diabetes Mellitus (IDDM): Patients. Journal of Periodontology 55:402-405.

21. Beal J. Social Factors and Preventive Dentistry. In: The Prevention of Dental Disease, Edited By Murray J, Oxford: Oxford Univ Press, 1989, p. 373-405.

22. Beck J and Hunt R (1985): Oral Health Status in the US: Problems of Special Patients. J Dent Educ 49:407-25.

23. Beck J, Kohout F, Hunt R, and Heckert D (1987): Root Caries: Physical, Mental, and Psychosocial Correlates in an Elderly Population. Gerodontics 3:242-47.

24. Beck JD (1984): The Epidemiology of Dental Diseases in The Elderly. Gerodont 3:5-15.

25. Beck JD, Katz RV, Page RC, Greene LC and Löe H (1987b): Joint AADS-IADR Symposium on Oral Health of US Adults-NIDR National Survey. Journal of Public Health Dentistry 47:198-205.

26. Beck JD, Koch GG, Zambon JJ, Genco RJ and Tudor GE (1992): Evaluation of Oral Bacteria as Risk indicators for Periodontitis in Older Adults. Journal of Periodontology 63:93-99.

27. Beck JD, Koch GG, Rozier GR, and Tudor GE (1990): Prevalence and Risk Indicators for Periodontal Attachment Loss in a Population of Older CommunityDwelling Blacks and Whites. Journal of Periodontology 61:521-528.

28. Becker W, Berg L, and Becker BE (1979): Untreated Periodontal Disease: A Longitudinal Study. Journal of Periodontology 50:234-44.

29. Belting CM, Hiniker JJ, and Dummett CO (1964): Influence of Diabetes Mellitus on the Severity of Periodontitis. Journal of Periodontology 35:476-80.

30. Bender IB and Seltzer S (1961): Roentgenographic and Direct Observation of Experimental Lesions in Bone II. JADA 62:708.

31. Bennet KR and Read PC (1982): Salivary Immunoglobulin A Level in Normal Subjects, Tobacco Smokers, and Patients with Minor Aphthous Ulcerations. Oral Surg, Oral Med, Oral Path 53:461-465.

32. Bergner M and Rothman ML (1987): Health Status Measures: An Overview and Guide for Selection. Annual Rev P Health 8:191-210. 
33. Bergstrom J and Eliasson S (1987): Cigarette Smoking and Alveolar Bone Height in Subjects With a High Standard of Oral Hygiene. J Clin Periodontol 14:466-69.

34. Bergstrom J and Floderus-Myrhed B (1983): Co-Twin Control Study of The Relationship Between Smoking and Some Periodontal Disease Factors. Commun Dent and Oral Epid 11:113-116.

35. Bergstrom J (1989): Cigarette Smoking as a Risk Factor in Chronic Periodontal Disease. Commun Dent and Oral Epid 17:245-247.

36. Bergstrom J and Eliasson S (1989): Prevalence of Chronic Periodontal Disease Using Probing Depth as a Diagnostic Test. J Clin Periodontol 16:588-592.

37. Bergstrom J, Eliasson S and Preber H (1991): Cigarette Smoking and Periodontal Bone Loss. Journal of Periodontology 62:242-246.

38. Bergstrom J, Pearson L and Preber, H (1988): Influence of Cigarette Smoking on Vascular Reaction During Experimental Gingivitis. Scand J Dent Res 96:34-39.

39. Bjorn H and Holmberg K (1966): Radiographic Determination of Periodontal Bone Destruction in Epidemiological Research. Odont Revy 17:232-250.

40. Blackmore JW (1994): Top Prescription Drugs. Oral Health Feb:11-17.

41. Blaikie DC (1979): Cultural Barriers To Preventive Dentistry. Aust Dent J 24:398401.

42. Blankenstein R, Murray JJ and Lind OP (1978): Prevalence of Chronic Periodontitis in 13-15-Year-Old Children: A Radiographic Study. J Clin Periodontol 5:285-92.

43. Bolden AJ, Henry JL and Allukian M (1993): Implications of Access, Utilization and Need for Oral Health Care by Low Income Groups and Minorities on the Dental Delivery System. J Dent Educ 57:888-98.

44. Borland BL and Rudolph JP (1975): Relative Effects of Low Socioeconomic Status, Parental Smoking, and Poor Scholastic Performance on Smoking Among High School Students. Social Science and Medicine 9:27-30.

45. Brown JL, Oliver RC and Löe H (1990): Evaluating Periodontal Status of US Employed Adults. JADA 121:226-232.

46. Brown L, Jackson and Löe H (1993): Prevalence, Extent, Severity and Progression of Periodontal Disease. Periodontology 2000 2:57-71.

47. Bullough B (1972): Poverty, Ethnic Identity and Preventive Health Care. Journal of Health and Social Behavior 13:347-59.

48. Burt B, Haugejorden O, Hein W, Horowitz H, Hunter P, Morrison E, and O'BrienMoran E (1984): The Prevention of Dental Caries and Periodontal Disease. Int Dent J 34:141-158. 
49. Burt BA. The Pattern of Periodontal Destruction in Industrialized Countries. In: Markers of Disease Susceptibility and Activity For Periodontal Diseases, Edited By Johnson N W, London Vol. \#3: Cambridge Univ Press, 1994, p. IX-464.

50. Burt BA. The Distribution of Periodontal Destruction in The Population of industrialized Countries. In: Risk Markers For Oral Diseases Vol 3 Periodontal Diseases, Edited By Johnson VW Ed, NY: Cambridge Univ Press, 1991, p. VII-464.

51. Burt BA (1993): Periodontology 2000 2:26-33.

52. Burt BA (1994): Periodontitis and Aging: Reviewing Recent Evidence. JADA 125:273-279.

53. Burt BA, Eklund SA, Landis JR, Larkin FA, Guire KE and Thompson FE (1982): Diet and Dental Health, A Study of Relationships, United States, 1971-74. National Center For Health Statistics, Vital and Health Statistics. PHS Series 11, Wash, DC: No. 225-PHS 82-1645.

54. Burt BA. Risk Markers For Oral Diseases, NY: Cambridge Press, 1991 pp. 9-26.

55. Calnan M (1985): Patterns in Preventive Behavior: A Study of Women in Middle Age. Social Science and Medicine 20:263-68 .

56. Campbell NM (1971): A Light and Electron Microscopy Study of Blood Vessels From Gingival Tissue of Non-Diabetic and Diabetic Patients. Aust Dent J 16:23539.

57. Caton J, Polson A, and Bouwsma O (1988): Associations Between Bleeding and Visual Signs of Interdental Gingival Inflammation. Journal of Periodontology 59:722-27.

58. Caton J. Consensus Report, Discussion Section I. Periodontal Diagnosis and Diagnostic Aids. in: Consensus Report American Academy of Periodontology, Edited By Nevins M, Becker W, and Kornman K. American Academy of Periodontology, 1993.

59. Chassin L, Presson CC, Sherman SJ, Corty E, and Olshavsky RW (1984): Predicting the Onset of Smoking in Adolescents: A Longitudinal Study. Journal of Applied Social Psychology 14:224-43.

60. Chen MA and Stone DB (1983): Toothbrushing, Flossing, and Dental Visits in Relation To Socioeconomic Characteristics of White American Families. Commun Dent and Oral Epid 11:325-332.

61. Ciancio SG (1986): Current Status of Indices of Gingivitis. J Clin Periodontol 13:375-78.

62. Cianciola LJ, Park BH and Bruck E (1982): Prevalence of Periodontal Disease in Insulin-Dependent Diabetes Mellitus (Juvenile Diabetes ):. JADA 104:653-60.

63. Clarke NG and Carey SE (1985); Etiology of Chronic Periodontal Disease: An Alternative Perspective. JADA 110:689-691. 
64. Clarke NG, Shepard BC and Hirsch RS (1981): The Effects of Intra-Arterial Epinephrine and Nicotine on Gingival Circulation. Oral Surg, Oral Med 52:577582.

65. Cleghorn, B. Personal Communication, 1994. (Unpub).

66. Clerehugh V and Lennon MA (1984): The Attachment Level As A Measure of Early Periodontitis. Community Dental Health 1:33-40.

67. Clerehugh V and Lennon MA and Worthington HV (1988): Aspects of The Validity of Buccal Loss of Attachment $\geq 1 \mathrm{~mm}$ in Studies of Early Periodontitis. J Clin Periodontol 15:207-210.

68. Coburn D and Pope CR (1974): Socioeconomic Status and Preventive Health Behavior. Journal of Health and Social Behavior 15:67-78.

69. Cohen DW, Friedman LA, Shapiro J, Kyle GC and Franklin S (1970): Diabetes Mellitus in Periodontal Disease: Two Year Longitudinal Observations. Journal of Periodontology 41:709-12.

70. Computer Keyes and Woodway WA, Data Entry Emulator, 1991 (Unpub).

71. Cornfield J, Gordon T, and Smith W (1961): Quantal Response Curves For Experimentally Uncontrolled Variables. Bull of International Statistical Institute 38:97-115.

72. Costabel U, Bross KJ, Reuter C, Ruhle KH, and Mattyhs H (1986): Alterations in Immunoregulatory T-Cell Subsets in Cigarette Smokers: A Phenotypic Analysis of Bronchoalveolar and Blood Lymphocytes. Chest 90:39-44.

73. Cramer EM (1972): Significance Tests and Tests of Models in Multiple Regression. American Statistician 26:26-29.

74. Cutress TW, Powell RN and Ball ME (1982): Differing Profiles of Periodontal Disease in Two Similar South Pacific Island Populations. Commun Dent and Oral Epid 10:193-203.

75. Danielson B, Manji F, Nagel Kerke N, Fejerskov O, and Baelum V (1990): Effect of Cigarette Smoking on The Transition Dynamics in Experimental Gingivitis. $J$ Clin Periodontol 17:159-164.

76. Davenport RH Jr., Simpson DM, and Hassell TM (1982): Histometric Comparison of Active and Inactive Lesions of Advanced Periodontitis. Journal of Periodontology 53:285-95.

77. Davies PH, Downer MC and Lennon MA (1978): Periodontal Bone Loss in English Secondary School Children: A Longitudinal Radiological Study. J Clin Periodontol 5:278-84.

78. Depart Health and Human Services (1989): Reducing The Health Consequences of Smoking: 25 Years of Progress: A Report of The Surgeon General. Wash DC DHHS NO CDC 89-8411:GPO.

79. Destefano F, Anda RF, Kahn HS, Williamson DF, and Russell CM (1993): Dental Disease and Risk of Coronary Heart Disease and Mortality. BMJ 306:688-691. 
80. Dielman TE, Leech S, Becker MH, Rosenstock IM, Horvath WJ and Radius SM (1982): Parental and Child Health Beliefs and Behaviors. Health Education Quarterly 9 (2\&3):60-156.

81. Dolan $\mathrm{T}$ and Atchison K (1993): Implications of Access, Utilization and Need For Oral Health Care by the Non-institutionalized and Institutionalized Elderly on the Dental Delivery System. J Dent Educ 57:876-87.

82. Doll R and Peto R (1976): Mortality in Relation To Smoking. BMJ 2:1525-1536.

83. Eichel B and Shahrik HA (1969): Tobacco Smoke Toxicity: Loss of Human Oral Leukocyte Function and Fluid Cell Metabolism. Science 166:1424-8.

84. Ellenberg $M$ and Rifkin H, Diabetes Mellitus: Theory and Practice, NY:McgrawHill Publ, 1970. pp. 734.

85. Emrich LJ, Shlossman M, and Genco RJ (1993): Periodontal Disease in NonInsulin Dependent Diabetes Mellitus. Journal of Periodontology.

86. Engelberger A, Rateitschak K, and Marthaler TM (1963): Zur Messung Des Parodontalen Knochenschwundes. Helv Odont Acta 7:34.

87. Engelberger T (1983): Correlations Among Papilla Bleeding Index, Other Clinical Indices and Histologically Determined Inflammation of Gingival Papilla. J Clin Periodontol 10:579.

88. Ettinger RL (1992): Attitudes and Values Concerning Oral Health and Utilisation of Services Among The Elderly. Int Dent J 42:373-384.

89. Evans AS (1978): Causation and Disease: A Chronological Journey. Am J Epidemiol 108:249-258.

90. Feldman RS, Bravzcos JS, and Rose CL (1983): Associations Between Smoking, Different Tobacco Products and Periodontal Disease Indexes. Journal of Periodontology 54:481-88.

91. Fleiss JL. Statistical Methods For Rates and Proportions, Toronto: John Wiley and Sons, 1981. Ed. 2.

92. Forbes WF and Thompson ME (1989): The Quantitative Evaluation of Risks: Unresolved Problems. Can J Public Health 80:282-90.

93. Frandson A, Public Health Aspects of Periodontal Disease: Proceedings of A Workshop, Chicago: Quintessence Publ, 1984. pp. 5-255.

94. Frazier PJ (1978): A New Look At Dental Health Education in Community Programmes. Dent Hygiene 52:176-86.

95. Frazier PJ and Horowitz AM (1990): Oral Health Education and Promotion in Maternal and Child Health: A Position Paper. Journal of Public Health Dentistry 50:390-95. 
96. Gelskey DE, Macdonald S, and Young TK. Highlights Report of The Manitoba Heart Health Survey, Winnipeg: Dept Community Health Sciences, Univ Manitoba, 1991.

97. Gelskey SC and Pruthi VK (1990): Localized Juvenile Periodontitis: A Review of The Literature. JCDA 56:791-95.

98. Gibbs CH, Hirshfeld JW, Lee JF, Low SB, Magnusson I, Thousand RR, Yerneni P, et al (1988): Description and Clinical Evaluation of A New Computerized Periodontal Probe - The Florida Probe. J Clin Periodontol 15:137-4.

99. Gift HC (1988): Awareness and Assessment of Periodontal Problems Among Dentists and the Public. Int Dent J 38:147-53.

100. Gift HC, Reisine ST, and Larach DC (1992): The Social Impact of Dental Problems and Visits. Am. J. Pub. Health 82:1663-1668.

101. Gilmour H, Day E, and Northridge D (1989): Dental Health and Acute Myocardial Infarction. BMJ 298:1579-1580.

102. Gjermo P, Bellini HT, Santos VP, Martins JG, and Ferracyoli JR (1984): Prevalence of Bone Loss In A Group of Brazilian Teenagers Assessed On BiteWing Radiographs. J Clin Periodontol 11:104-13 .

103. Glanz K, Lewis F, and Rimer B. Health Behavior and Health Education: Theory Research and Practice, San Francisco: Jossey-Bass Publ, 1991. pp. IX-460.

104. Glass R, Johnson B, and Vessey M (1974): Accuracy of Recall of Histories of Oral Contraceptive Use. Brit J Prev Soc Med 28:273-75.

105. Goodson JM. Selection of Suitable Indicators of Periodontitis. In: Risk Assessment: In Dentistry:Proceedings of a Conference, Edited By Bader JD, Chapel Hill: Univ. N. Carolina, 1990, p. 69-74.

106. Goodson JM (1986): Clinical Measurements of Periodontitis. J Clin Periodontol 13:446-455.

107. Goodson JM, Haffajee AD, and Socransky SS (1984): The Relationship Between Attachment Level Loss and Alveolar Bone Loss. J Clin Periodontol 11:348-59.

108. Goodson JM, Taner AC, Haffajee AD, Sornberger GC, and Socransky SS (1982): Patterns of Progression and Regression of Advanced Periodontal Disease. J Clin Periodontol 9:472-481.

109. Goultschiin J, Sgan Cohen JD, Honchin M, Brayer L, and Soskolne WA (1990): Association of Smoking With Periodontal Treatment Needs. Journal of Periodontology 61:364-367.

110. Grant D and Bernick S (1970): Arteriosclerosis In Periodontal Vessels of Aging Humans. Journal of Periodontology 41:170-173.

111. Graves R, Bohannan H, Disney J, Stamm J, and Bader J (1986): Recent Dental Caries and Treatment Patterns in US Children. Journal of Public Health Dentistry 46:23-29. 
112. Grbic JT, Lamster IB, Romanita S, Fine C, and Fine JB (1991): Risk Indicators for Future Clinical Attachment Loss in Adult Periodontitis. Patient Variables. Journal of Periodontology 62:322-29.

113. Greene JC. General Principles of Epidemiology and Methods For Measuring Prevalence and Severity of Periodontal Disease. In: Contemporary Periodontics, Edited By Genco RJ, Goldman HM, and Cohen DW. St. Louis: Mosby, 1990, p. 97105.

114. Greenstein G, Caton J, and Polson AM (1981): Histologic Characteristics Associated With Bleeding After Probing and Visual Signs of Inflammation. Journal of Periodontology 52:420-425.

115. Griffiths GS, Wilton JM, Curtis MA, Maiden MF, Gillett IR, Wilson DT, Sterne JA, et al (1988): Detection of High-Risk Groups and Individuals For Periodontal Diseases: Clinical Assessment of the Periodontium. J Clin Periodontol 15:403-410.

116. Haber J and Kent RL (1992): Cigarette Smoking in a Periodontal Practice. Journal of Periodontology 63:100-106.

117. Haber J, Wattles J, Crowby M, Mandell R, Kaunuki J, and Kent R (1993): Evidence For Cigarette Smoking as a Major Risk Factor For Periodontitis. Journal of Periodontology 64:16-20.

118. Haefner DP, Kegeles SS, Kirscht J, and Rosenstock IM (1967): Preventive Actions In Dental Disease, Tuberculosis and Cancer. Public Health Reports 82:451-59.

119. Haffajee AD and Socransky SS (1986): Attachment Level Changes In Destructive Periodontal Disease. J Clin Periodontol 13:461.

120. Haffajee AD, Socransky SS, and Goodson JM (1982): Periodontal Disease Activity. J Periodontal Res 17:521-22.

121. Haffajee AD, Socransky SS, and Goodson JM (1983): Comparison of Different Data Analyses For Detecting Changes In Attachment Level. J Clin Periodontol 10:298310.

122. Haffajee AD, Socransky SS, Lindhe J, Kent RL, Okamoto H, and Yoneyama T (1993): Clinical Risk Indicators For Periodontal Attachment Loss. J Clin Periodontol.

123. Hansen BF, Gjermo P, and Bergwitz-Larsen KR (1984): Periodontal Bone Loss In 15-Year-Old Norwegians. J Clin Periodontol 11:125-31.

124. Harris DM and Guten S (1979): Health Protective Behavior: An Exploratory Study. Journal of Health and Social Behavior 20:17-29.

125. Hassard T. Advanced Biostatistics, 1992. (Unpub).

126. Hausmann E, Allen K, and Clerehugh V (1991): What Alveolar Crest Level On A Bite-Wing Radiograph Represents Bone Loss? Journal of Periodontology 62:570-72.

127. Hayward R, Meetz H, Shapiro M, and Freeman H (1994): Utilization of Dental Services: 1986 Patterns and Trends. Journal of Public Health Dentistry. 
128. Health and Welfare Canada, Main Findings Report of The Canadian Blood Pressure Survey, Ottawa, 1989.

129. Heifetz SB and Suomi JD (1973): The Control of Dental Caries and Periodontal Diseases: A Fundamental Approach. Journal of Public Health Dentistry 33:2-6.

130. Hennekens $\mathrm{CH}$ and Buring JE. Epidemiology In Medicine, Toronto: Little, Brown and Co, 1987. pp. VII-383.

131. Hildebolt CF, Rupich RC, Vannier MW, Zerbolio DJ, Shrout MK, Cohen S, and Pinkas A (1993): Inter-Relationships Between Bone Mineral Content Measures: Dual Energy Radiography (DER) and Bitewing Radiographs (BWX). J Clin Periodontol 20:739-45.

132. Hill AB (1965): The Environment and Disease: Association Or Causation? Proc Roy Soc Med 58:295-300.

133. Hintze JL. Number Cruncher Statistical System Product, 5.3, 1992. (Unpub).

134. Hirschmann PN (1987): Radiographic Interpretation of Chronic Periodontitis. Int Dent J 37:3-9.

135. Hollister M and Weintraub J (1993): The Association of Oral Status With Systemic Health, Quality of Life, and Economic Productivity. J Dent Educ 57:901-12.

136. Hoover JN and Mcdermott RE (1990): Dental Treatment Needs of Elderly Patients Seen at a University Teaching Clinic. Am J Dent 3:213-16.

137. Horning GM Hatch CL, and Cohen ME (1992): Risk Indicators For Periodontitis In A Military Treatment Population. Journal of Periodontology 63:297-302.

138. Hugson A and Jordan T (1982): Frequency Distribution of Individuals Aged 20-70 Years According To Severity of Periodontal Disease. Commun Dent and Oral Epid 10:187-92.

139. Hugson A, Thorstensson H, Falk H, and Kuylensticrna J (1989): Periodontal Conditions In Insulin-Dependent Diabetes. J Clin Periodontol 16:215-223 .

140. Hull PS, Hillam DG, and Beal JF (1975): A Radiography Study of The Prevalence of Chronic Periodontitis In 14-Year-Old English School Children. J Clin Periodontol 2:203.

141. Hunt RJ (1988): Is It Time To Reassess The Public Health Implications of Periodontal Disease? A Review of Current Concepts. Journal of Public Health Dentistry 48:241-44.

142. Hunt RJ, Field HM, Beck JD (1985): The Prevalence of Periodontal Conditions in a Non-Institutionalized Elderly Population. Gerodontics 1:176-180.

143. Hunt JH, Levy SM and BecK JD (1990): The Prevalence of Periodontal Attachment Loss in an Iowa Population Aged 70 and Older. Journal of Public Health Dentistry 50:251-256.

144. Imrey PB (1986): Considerations In The Statistical Analysis of Clinical Trials In Periodontitis. J Clin Periodontol 13:517-528. 
145. Ismail AI, Burt BA, and Eklund SE (1983): Epidemiologic Patterns of Smoking and Periodontal Disease in the United States. JADA 106:617-21.

146. Ismail AI and Lewis DW (1993): Periodic Health Examination, 1993 Update: 3. Periodontal Diseases: Classification, Diagnosis, Risk Factors and Prevention. Can Med Assoc J 149:1409-1422.

147. Ismail AI, Burt BA, and Eklund SE (1983): Epidemiologic Patterns of Smoking and Periodontal Disease In The United States. JADA 106;617-623.

148. Janssen PTM and Van Palenstein-Halderman WH (1985): Analysis of Successive Bleeding Scores in the Individual Patient. IADR/AADR Abstract 785.

149. Jeffcoat MK, Jeffcoat RL, Jens SC, and Captain K (1986): A New Periodontal Probe With Automated Cemento-Enamel Junction Detection. J Clin Periodontol 13:27680 .

150. Jeffcoat MK and Reddy MS (1991): Progression of Probing Attachment Loss In Adult Periodontitis. Journal of Periodontology 62:185-189.

151. Jeffery R (1989): Risk Behaviors and Health. American Psychologist 44:1194-1202.

152. Jenkins WM and Mason WN (1984): Radiographic Assessment of Periodontitis. British Dental Journal 156:170-74.

153. Jensen J, Lijemark W, and Bloomquist C (1981): The Effect of Female Sex Hormones On Subgingival Plaque. Journal of Periodontology 52:599-602.

154. Joffres Mr, Hamet P, and Rabkin SW, (1992):Prevalence, Control and Awareness of High Blood Pressure Among Canadian Adults. CMAJ 146:1997-2005

155. Kahn HA and Sempos CT. Statistical Methods In Epidemiology, NY: Oxford Univ Press, 1989. pp. x-292.

156. Kaimenyi JT and Ashley FP (1988): Assessment of Bone Loss In Periodontitis From Panoramic Radiographs. J Clin Periodontol 15:170-74.

157. Kalkwarf KL (1978): Effect of Oral Contraceptive Therapy On Ginigival Inflammation In Humans. Journal of Periodontology 49:560-63.

158. Kallestal C and Matsson L (1991): Marginal Bone Loss In 16-Year-Old Swedish Adolescents In 1975 and 1988. J Clin Periodontol 18:740-43.

159. Kasl SV and Cobb S (1966): Health Behavior, Illness Behavior and Sick Role Behavior. Archives of Environmental Health 12:246-266 and 531-770.

160. Kelly JE and Harvey T (1979): National Center For Health Statistics: Basic Data On Dental Examination Findings of Persons 1-74 Years. GPO US 1971-74, Series11:Vital \& Health Stats-No 214, Washington, DC.

161. Kelly JE and Van Kirk LE (1965): Periodontal Disease In Adults. National Center For Health Statistics. US 1960-62 Vital and Health Statistics. Series 11 and 12. PHS Publ No 1000 Washington, DC, GPO 11-12:1 
162. Kelsey JL, Thompson WD and Evans AS. Methods In Observational Epidemiology: Monographs In Epidemiology and Biostatistics, NY:Oxford Univ Press, 1986. Pp. 3-366.

163. Kenny E, Kraal HJ, Saxe SR, and Jones J (1977): The Effect of Cigarette Smoke On Human Oral Polymorphonuclear Leukocytes. J Periodontal Res 12:227-234.

164. Kidder LH and Judd CM. Questionnaires and Interviews: Overview of Strategies. In: Research Methods In Social Relations, Edited By Kidder LH, and Judd CM, NY: Rhinehart and Winston, 1986, P. 219-235.

165. Kingman A, Löe H, Anerud A, and Boysen H (1991): Errors In Measuring Parameters Associated With Periodontal Health and Disease. Journal of Periodontology 62:477-486.

166. Kleinbaum DG, Kupper LL and Morgenstern H. Types of Epidemiologic Research. In: Epidemiologic Research: Principles and Quantitiative Methods, Edited By Kleinbaum DG, Belmont, CA: Wadsworth Inc, 1982, P. 40-50.

167. Knight GM and Wade B (1973): The Effects of Hormonal Contraceptives On The Human Periodontium. Journal of Periodontology 9:18-22.

168. Kraal JH and Kenney EF (1979): The Response of Polymorphonuclear Leukocytes To Chemotatic Stimulation For Smokers and Non-Smokers. J Periodontal Res 14:383-89.

169. Lang N. Current Status of Non-surgical and Surgical Periodontal Treatment. In: Public Health Aspects of Periodontal Disease, Edited By Frandsen A, Chicago: Quintessence Publ, 1984, P. 217-229.

170. Last JM. A Dictionary of Epidemiology, Toronto: Oxford Univ Press, 1988. Ed. 2.

171. Leake J (1984): Expenditures On Dental Services In Canada, Canadian Provinces and Territories 1960-80. JCDA 50:362-68.

172. Leblond GT and Cobb DF. Lotus 2.0, 1985. (Unpub)

173. Lederman D, Lumerman H, Reuben S, and Feedman PD (1984): Gingival Hyperplasia Associated With Nifedipine Therapy. Oral Surg, Oral Med, Oral Path $57: 620-22$.

174. Levy SM, Heckert DA, Beck JD, and Kohout FJ (1987): Multivariate Correlates of Periodontally Healthy Teeth In An Elderly Population. Gerodontics 3:85-88.

175. Lightner LM, O'Leary TJ, Drake RB, Crump PP, and Allen MF (1971): Preventive Periodontic Treatment Procedures: Results Over 46 Months. Journal of Periodontology 42:555-61.

176. Lindhe J, Hamp SE, and Löe H (1973): Experimental Periodontitis In The Beagle Dog. J Periodontal Res 8:1-10.

177. Listgarten MA (1972): Normal Development, Structure, Physiology and Repair of Gingival Epithelium. Oral Sciences Reviews 1:3-67. 
178. Listgarten MA (1980): Periodontal Probing: What Does It Mean? J Clin Periodontol 7:165-76.

179. Locker D (1988): Measuring Oral Health: A Conceptual Framework. Community Dental Health 5:3-18.

180. Locker D. An Introduction To Behavioural Science and Dentitsry, NY:Tavistock/Routledge, 1989. Pp. IX-259.

181. Locker D (1992): The Burden of Oral Disorders In A Population of Older Adults. Community Dental Health 9:109-24.

182. Locker D and Grushka M (1987): Prevalence of Oral and Facial Pain and Discomfort: Preliminary Results of A Mail Survey. Commun Dent and Oral Epid 15:169-72.

183. Locker D, Leake JL, Lee J, Main PA, Hicks T, and Hamilton M, (1991): Utilization of Dental Services By Older Adults In Four Ontario Communities. JCDA 57:879-86.

184. Locker D. Aging and Oral Health. In: An Introduction To Behavioural Science and Dentistry, Edited By Locker D, NY: Tavistock, 1989, P. 59-72.

185. Locker D, and Leake JL (1993): Risk Indicators and Risk Markers For Periodontal Disease Experience In Older Adults Living Independently In Ontario, Canada. Journal of Dental Research 72:9-17.

186. Löe H, Anerud A, Boysen H, and Morrison E (1986): Natural History of Periodontal Disease In Man. Rapid, Moderate and No Loss of Attachment In Sri Lankan Laborers 14-46 Years of Age. J Clin Periodontol 13:431-440.

187. Löe H, Anerud A, Boysen H, and Smith M (1978): The Natural History of Periodontal Disease In Man. The Rate of Periodontal Destruction Before 40 Years of Age. Journal of Periodontology 49:607-620.

188. Löe H and Silness J (1963): Periodontal Disease In Pregnancy. Acta Odont Scand 21:533-51.

189. Löe H, Theilade E, and Jensen SB (1965): Experimental Gingivitis In Man. Journal of Periodontology 36:177-87.

190. Lovdal A, Arno A, and Schei A (1961): Combined Effect of Subgingival Scaling and Controlled Oral Hygiene on the Incidence of Gingivitis. Acta Odont Scand 19:537555 .

191. Macgregor IDM (1984): Toothbrushing Efficiency In Smokers and Non-Smokers. $\mathrm{J}$ Clin Periodontol 11:313-20.

192. Machtei EE, Christersson SG, Grossi SG, Dunford R, Zambon JJ, and Genco RJ (1992): Clinical Criteria For The Definition of "Established Periodontitis". Journal of Periodontology 63:207-215.

193. Macmahon B and Pugh TF. Epidemiology: Principles and Methods, Boston:Little, Brown Co, 1970. 
194. Mandel ID (1974): Indices For Measurement of Soft Accumulations In Clinical Studies of Oral Hygiene and Periodontal Disease. J Periodontal Res 9 Supp 14:7-30.

195. Manning W, Bailit H, Benjamin B, and Newhouse J. The Demand For Dental Care: Evidence From A Randomized Trial In Health Insurance, Santa Monica:Rand, 1986.

196. Manouchehr-Pour M, and Bissada NF (1983): Periodontal Disease In Juvenile and Adult Diabetic Patients: A Review of The Literature. JADA 107:766-770.

197. Mantel N and Haenszel W (1959): Statistical Aspects of the Analysis of Data from Retrospective Studies of Disease. Journal of The National Cancer Institute 22:71948.

198. Matilla KJ, Bieminen MS, Valtonen VV, Rasi RP, Kesaniemi YA, Syrajala SL, et al. (1989): Association Between Dental Health and Acute Myocardial Infarction. BMJ 298:779-781.

199. Matsson L, Attstrom R, and Granath L (1980): Inflammatory Reactions in the Gingival Region During the Development of Bacterial Plaque in Alloxan-Diabetic Rats. J Periodontal Res 15:516-24.

200. McDowell I and Newell C. Measuring Health: A Guide To Rating Scales and Questionnaires, NY:Oxford Univ Press, 1987.

201. Millar WG and Wigle DT (1986): Socioeconomic Disparities In Risk Factors For Cardiovascular Disease. Can Med Assoc J 134:127-32.

202. Miller AJ, Brunelle HA, Carlos JP, Brown LJ, and Löe H (1987): Oral Health of United States Adults; The National Survey of Oral Health In US Employed Adults and Seniors 1985-86. NIH 87-2868:Washington-NIH.

203. Ministry of Supply and Services, Canada Health Survey: The Health of Canadians, Ottawa:Ministry of Supp and Ser, 1981.

204. Nathanson CA (1977): Sex Roles as Variables in Preventive Health Behavior. Journal of Community Health 3:142-155.

205. Newman HN (1990): Plaque and Chronic Inflammatory Periodontal Diseases: A Question of Ecology. J Clin Periodontol 17:533-41.

206. Nichols S, Laster L, and Bodak-Gyoval L (1978): Diabetes Mellitus and Periodontal Disease. Journal of Periodontology 49:85-88.

207. Nikias MK, Fink R and Shapiro S (1975): Comparisons of Poverty and Nonpoverty Groups On Dental Status, Needs, and Practices. Journal of Public Health Dent 35:237-258.

208. Nikiforuk G. Understanding Dental Caries: Etiology and Mechanisms Basic and Clinical Aspects, N.Y.: Karger, 1985. Pp. 80.

209. Norman RMG. The Nature and Correlates of Health Behavior, Ottawa:Health and Welfare Canada, 1986. Pp. 1-163. 
210. Nysether S and Hanson F (1983): Errors On Dental Bitewing Radiographs. Commun Dent and Oral Epid 11:286-88.

211. O'Leary TJ, Drake RB, and Naylor JE (1972): The Plaque Control Record. Journal of Periodontology 43:38.

212. Okamoto H, Yoneyama T, Lindhe J, and Socransky S, (1988): Methods of Evaluating Periodontal Disease Data In Epidemiological Research. J Clin Periodontol 15:430-39.

213. Oliver RC, Brown L Jackson, and Löe H (1993): Periodontal Treatment Needs. Periodontology 2000 2:150-160.

214. Page RC (1984): Periodontal disease in the elderly. Gerodontology 3:63-70.

215. Page RC (1986): Gingivitis. J Clin Periodontol 13:345-355.

216. Page RC (1995): Critical Issues in Periodontal Research. J Dent Res 74:1118 - 28.

217. Page RC, Altman LC, and Ebersole JL (1983): Rapidly Progressive Periodontitis: A Distinct Clinical Condition. Journal of Periodontology 54:197-209.

218. Page RC and Schroeder HE (1982): Current Status of The Host Response In Chronic Marginal Periodontitis. Journal of Periodontology 52:477-491.

219. Papapanou PN, Wennstrom JL and Grondahl K (1988): Periodontal Status In Relation To Age and Tooth Type: A Cross-Sectional Radiographic Study. J Clin Periodontol 15:469-78.

220. Papapanou PN, Wennstrom JL and Johnsson T (1991): Extent and Severity Index Based on Assessments of Radiographic Bone Loss. Commun Dent and Oral Epid 19:313-17.

221. Patel JR and Wuehrmann AH (1976): A Radiographic Study of Nutrient Canals. Oral Surg, Oral Med 42:693-701.

222. Patni VM, Merchant GJ, and Dhooria HS (1985): Incidence of Nutrient Canals in Hypertensive Patients. Oral Surg, Oral Med 59:206-211.

223. Payne BJ and Locker D (1992): Oral Self-Care Behavior in Older Dentate Adults. Commun Dent and Oral Epid 20:376-380.

224. Peristein MI and Bissada NB (1977): Influence of Obesity and Hypertension on The Severity of Periodontitis in Rats. Oral Surg, Oral Med 43:707-719.

225. Persson I, Bergkvist L, and Adami HO (1987): Reliability of Women's Histories of Climacteric Oestrogen Treatment Assessed By Prescription Forms. Int J Epidemiol 16:222-28.

226. Pihlstrom Bl, Ortiz-Campos C, and McHugh RB (1981): A Randomized Four-Year Study of Periodontal Therapy. Journal of Periodontology 52:227-242.

227. Pilot T (1986): Economic Perspectives on Diagnosis and Treatment Planning In Periodontology. J Clin Periodontol 13:889-93. 
228. Pilot $\mathrm{T}$ and Miyazaki $\mathrm{H}$ (1991): Periodontal Conditions In Europe. $J$ Clin Periodontol 18:353-57.

229. Pless IB and Miller JR (1979): Apparent Validity of Alternative Survey Methods. Journal of Community Health 5:22.

230. Polson AM, Caton JG, Yeaple RN and Zander HA (1980): Histological Determination of Probe Tip Penetration Into Gingival Sulcus of Humans Using An Electronic Pressure-Sensitive Probe. J Clin Periodontol 7:479.

231. Polson AM and Goodson JM (1985): Periodontal Diagnosis. Current Status and Future Needs. Journal of Periodontology 56:25-34.

232. Pratt L (1972): Conjugal Organization and Health. J Marriage and Family 34:8595.

233. Preber H and Bergstrom J (1985): Occurrence of Gingival Bleeding in Smoker and Non-Smoker Patients. Acta Odont Scand 43:315-320.

234. Preber $\mathrm{H}$ and Bergstrom J (1986): Cigarette Smoking in Patients Referred For Periodontal Treatment. Scand J Dent Res 94:102-108.

235. Preber H and Bergstrom J (1990a): Cigarette Smoking in Patients Referred For Periodontal Treatment. J Clin Periodontol 17:324-28.

235. Preber $\mathrm{H}$ and Bergstrom J (1990b): Effect of Cigarette Smoking on Periodontal Healing Following Surgical Therapy. J Clin Periodontol 17:324-328.

237. Preber H, Bergstrom J, and Linder L (1992): Occurrence of Periopathogens in Smoker and Non-Smoker Patients. J Clin Periodontol 19:667-671.

238. Quintarelli G (1957): Histopathology of The Human Mandibular Artery and Arterioles In Periodontal Disease. Oral Surg, Oral Med, Oral Path 10:1047-59.

239. Ramadan AB and Mitchell DF (1962): A Roentgenographic Study of Experimental Bone Destruction. Oral Surg, Oral Med, Oral Path 15:934-943.

240. Ramamurthy NS and Golub LM (1983): Diabetes Increases Collagenase Activity In Extracts of Rat Gingiva and Skin. J Periodontal Res 18:23-30.

241. Ramamurthy NS, Siegel M, Iacono VJ, and Golub LM (1979): Leukocyte Response In The Ginigival Crevice of The Diabetic Rat. J Periodontal Res 14:289-96.

242. Ramfjord SP (1987): Maintenance Care For Treated Periodontitis Patients. J Clin Periodontol 14:433-37.

243. Raulin L, McPherson J, McQuade M, and Hanson B (1989): The Effect of Nicotine On The Attachment of Human Fibroblasts To Glass and Human Root Surfaces In Vitro. Journal of Periodontology 59:318-325.

244. Reddy J, Africa CW, and Parker JR (1986): Darkfield Microscopy of Subgingival Plaque of An Urban Black Population With Poor Oral Hygiene. J Clin Periodontol 13:578-582. 
245. Reed BE and Polson AM (1984): Relationships Between Bitewing and Periapical Radiographs In Assesing Crestal Alveolar Bone Levels. Journal of Periodontology 55:22-27.

246. Reeder BA Angel A, and Ledoux M (1992): Obesity and Its Relation To Cardiovascular Disease Risk Factors In Canadian Adults. CMAJ 146:2009-19.

247. Rosenstock IM (1966): Why People Use Health Services. Milbank Memorial Fund Quarterly 44:94-124.

248. Rosenthal IM, Abrams H, and Kopczyk RA (1988): The Relationship of Inflammatory Periodontal Disease To Diabetic States in Insulin-Dependent Diabetes Mellitus Patients. J Clin Periodontol 15:425-429.

249. Rothman KJ. Modern Epidemiology, Toronto:Little Brown and Co, 1986. Pp. VII358.

250. Rundall TG and Wheeler JRC (1979): The Effect of Income on Use of Preventive Care: An Evaluation of Alternative Explanations. Journal of Health and Social Behavior 20:397-406.

251. Rundgren A and Mellstrom D (1984): The Effect of Tobacco Smoke On The Bone Mineral Content of The Aging Skeleton. Mech Aging Dev 28:273-77.

252. Rvasti T, Knuuttila M, Pohjamo L, and Haukipuor K (1985): Relation Between Control of Diabetes and Gingival Bleeding. Journal of Periodontology 56:154-57.

253. Ryan RJ (1985): The Accuracy of Clinical Paramenters In Detecting Periodontal Disease Activity. JADA 111:753-60.

254. Sackett DL (1979): Bias In Analytic Research. J Chron Dis 32:51-63.

255. Salloway JC and Dillon PD (1973): A Comparison of Family Networks and Friend Networks In Health Care Utilization. J Comparative Family Studies 4:131-142.

256. Sandler HC and Stahl SS (1960): Prevalence of Periodontal Disease In A Hospitalized Population. Journal of Dental Research 39:439-49.

257. Sartwell PE (1974): Retrospective Studies- A Review For The Clinican. Annals of Internal Medicine 81:381-86.

258. Sastrowijota SH, van der Velden U, Van Steenbergen TJ, Hillemaus P, Hart AA, De Graff J, and Abraham-Inpiju L (1990): Improved Metabolic Control, Clinical Periodontal Status and Subgingival Microbiology in Insulin-Dependent Diabetes Mellitus. A Prospective Study. J Clin Periodontol 17:233-242.

259. Sastrowijoto SH, Abbas F, Abraham-Inpijn L, and van der Velden U (1990): Relationship Between Bleeding/Plaque Ratio, Family History of Diabetes Mellitus and Impaired Glucose Tolerance. J Clin Periodontol 17:55-60.

260. Schei O, Waerhaug J, Lovdal A, and Arno A (1959): Alveolar Bone Loss as Related to Oral Hygiene and Age. Journal of Periodontology 30:7-16.

261. Schlesselman JJ. Case-Control Studies: Design, Conduct, Analysis, NY:Oxford Univ Press, 1982. Pp. VII-354. 
262. Selikowitz HS, Sheiham A, Albert D, and Williams GM (1981): Retrospective Longitudinal Study of The Rate of Alveolar Bone Loss in Humans Using BiteWing Radiographs. J Clin Periodontol 8:421-38.

263. Seymour RA and Heasman PA (1988): Drugs and the Periodontium. J Clin Periodontol 15:1-16.

264. Shanley D. Efficacy of Treatment Procedures in Periodontics, Chicago:Quintessence Publ, 1980. pp. 7-341.

265. Sheiham A (1991): Public Health Aspects of Periodontal Diseases in Europe. J Clin Periodontol 18:362-69.

266. Sheihiam A (1971): Periodontal Diseases and Oral Cleanliness in Tobacco Smokers. Journal of Periodontology 42:259-63.

267. Shortell SM and Richardson WC. Data Collection: Issues and Strategies. In: Health Program Evaluation, Edited By Shortell SM and Richarson WC, St Louis: CV Mosby Co, 1978, p. 84-91.

268. Shrout MK, Hildebolt CF, Vannier MW, Province M and Vahey EP (1990): Periodontal Disease Morbidity Quantification. I. Optimal Selection of Teeth for Periodontal Bone Loss Surveys. Journal of Periodontology 61:618-622.

269. Shrout PE and Fleiss JJ (1979): Intraclass Correlations: Uses in Assessing Rater Reliability. Psychological Bull 86:420-28.

270. Silness P and Löe H (1964): Periodontal Disease in Pregnancy II. Acta Odont Scand 22:121-35.

271. Simpson EH (1951): The Interpretation of Interaction in Contingency Tables. $\mathrm{J}$ of The Royal Statistical Society 13:238-41.

272. Snedecor GW and Cochran WG. Statistical Methods, AMES, Iowa:Iowa State Univ Press, 1980. Ed. 7

273. Socransky SS (1977): Microbiology of Periodontal Disease-Present Status and Future Considerations. Journal of Periodontology 48:497.

274. Soh G (1993): Racial Differences in Perception of Oral Health and Oral Health Behaviours in Singapore. Int Dent J.

275. Sooriyamoorthy M and Gower DB (1989): Hormonal Influences on Gingival Tissue: Relationship to Periodontal Disease. J Clin Periodontol 16:201-8.

276. Stachenko SJ, Reeder BA, Lindsay E, et al (1992): Smoking Prevalence and Associated Risk Factors in Canadian Adults. CMAJ 146:20-27.

277. Stahl SA and Fox LM (1953): Histologic Changes of Oral Mucosa Associated with Certain Chronic Diseases. Oral Surg, Oral Med, Oral Path 6:339-344.

278. Statistics Canada (1993): Income Distribution by Size in Canada. 13-207. Ottawa. 
279. Stolley PD, Tonascia JA, Sartwell PE, Tockman MA, Tonascia S, Rutledge A, and Schinnar R (1978): Agreement Rates Between Oral Contraceptive Users and Prescribers in Relation to Drug Use Histories. Am J Epidemiol 107:226-35.

280. Stoner JE (1972): An Investigation into the Accuracy of Measurements Made on Radiographs of the Alveolar Crests of Dried Mandibles. Journal of Periodontology 43:699-701.

281. Suomi JD, Plumbo J, and Barbano JP (1968): A Comparative Study of Radiographs and Pocket Measurements in Periodontal Disease Evaluation. Journal of Periodontology 39:311-15.

282. Suzuki JB (1988): Diagnosis and Classification of the Periodontal Diseases. Dent Clin North Am 32:195-216.

283. Sweet JB and Butler DP (1979): The Relationship of Smoking to Localized Osteitis. $J$ of Oral Surgery 37:732-35.

284. Syrajala AM, Knuuttila ML, and Syrjala LK (1992): Reasons Preventing Regular Dental Care. Commun Dent and Oral Epid 20:10-14.

285. Tabachnick BG and Fidell LL. In: Using Multivariate Statistics, NY: HarperCollins, 1989.

286. Tervonen T and Knuuttila M (1986): Relation of Diabetic Control to Periodontal Pocketing and Bone Loss. Oral Surg, Oral Med, Oral Path 61:346-49.

287. Theil EM and Heany TG (1991): The Valididy of Periodontal Probing as a Method of Measuring Loss of Attachment. J Clin Periodontol 18:648-653.

288. Thomason JM, Seymour RA, and Rice N (1993): The Prevalence and Severity of Cyclosporin and Nifedipine-Induced Gingival Overgrowth. J Clin Periodontol 20:37-40.

289. US Department of Health and Human Services (1992): A Framework for Assessing the Effectiveness of Disease and Injury Prevention. Morbidity and Mortality Weekly Report 41:1-12.

290. van der Velden U (1982): Location of Probe Tip in Bleeding and Non-Bleeding Pockets with Minimal Gingival Inflammation. J Clin Periodontol 9:421-27.

291. van der Velden U (1978): Errors in the Assessment of Pocket Depth In Vitro. J Clin Periodontol 5:182-87.

292. van der Velden U (1994): Effect of Age on the Periodontium. J Clin Periodontol.

293. van der Velden U, and De Vries JH (1978): Introduction of a New Periodontal Probe: The Pressure Probe. J Clin Periodontol 5:188-197.

294. van der Velden U (1991): The Onset Age of Periodontal Destruction. J Clin Periodontol 18:380-383.

295. Walden HB (1988): The Elderly Lead in Seeking Treatment. The Elderly are Leading in the Increasing Use of Dental Services During the Second Half of the 1980's. J Am Coll Dent 55:36-42. 
296. Wall BF, Fisher EA and Paynter R (1979): Dose to Patients from Pantomographic and Conventional Dental Radiography. British Journal of Radiology 52:727.

297. Weintraub JA and Burt BA (1985): Oral Health Status in the United States: Tooth Loss and Edentulism. J Dent Educ 49:368-76.

298. WHO (1970): Fluorides and Human Health. WHO Monograph No. 59:WHOGeneva.

299. WHO (1982): A Review of Current Recommendations for the Organization and Administration of Community Oral Health Services in Northern and Western Europe. Reports of a WHO Workshop, Copenhagen: WHO Regional office For Europe. WHO.

300. Williams AF (1972): Personality Characteristics Associated with Preventive Dental Health Practices. Journal of American College of Dentists 39:225-34.

301. Williams AF and Wechsler H (1972): Interrelationships of Preventive Actions in Health and Other Areas. Health Services Reports 87:969-76.

302. Wilson RW and Elinson J (1981): National Survey of Personal Health Practices and Consequences: Background, Conceptual Issues and Selected Findings. Public Health Reports 96:218-225.

303. Wilton JMA, Griffiths GS, Curtis MA, Maiden MFJ, Gillett IR, Wilson DT, Sterne JAC, et al (1988): Detection of High Risk Groups and Individuals for Periodontal Diseases. J Clin Periodontol 15:339-46.

304. Young TK, Gelskey DE, Macdonald SM, Hook E, and Hamilton S (1991): The Manitoba Heart Health Survey: Technical Report. Dept. Community Health Sciences, Fac of Medicine:Univ of Manitoba.

305. Zimmet PZ (1992): Kelly West Lecture 1991 - Challenges in Diabetic Epidemiology - from West to the Rest. Diabetes Care 15:232-52. 


\section{APPENDICES}


FACULTY OF DENTISTRY

Office of the Dean
730 Bannatyne Avenue

Winnipeg, Manitoba

Canada R3E OW2

Tel: (204) 789-3631

Fax: (204) $888-4113$

November 10, 1994

Dear Mr./Mrs./Ms

Professor S.C. Gelskey of the Faculty of Dentistry is conducting a study of patients who have received dental care at the Faculty of Dentistry.

The purpose of the study is to provide information which will help the Faculty continue to plan programs which best meet the needs of our patients. The Faculty of Dentistry fully supports the purpose and the methods of this study. Your participation in the study would be appreciated.

You, along with approximately 400 of our other patients, have been selected at random from our current patient registry to participate in a 30 minute telephone interview. Your answers to the interview will be kept strictly confidential.

You will be called within the next few weeks. At that time, you will be asked if you are willing to participate in the telephone interview.

If, for any reason, you do not wish to participate in the interview or you do not want to answer certain questions in the interview, simply tell the interviewer who telephones you.

You are under no obligation to participate in the study and your refusal will not influence any future care that you receive at the Faculty of Dentistry.

If you have any questions, please contact:

Professor S.C. Gelskey

Dental Diagnostic \& Surgical Sciences

Faculty of Dentistry

Phone:

Sincerely,

JU氏́. Wright, DDS, MSCD

Dean, Faculty of Dentistry
B. Cleghorn, DMD, MSc

Head of Clinics and Patient Care 


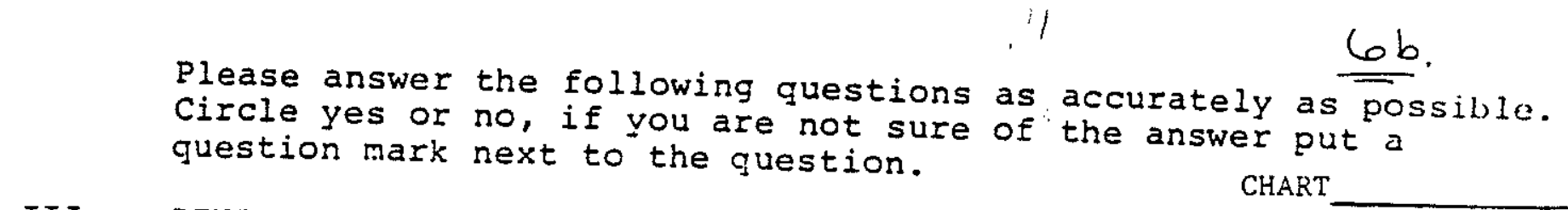

III. DENTAL HISTORY

DENTIST'S NAME/ADDRESS

PHONE

Date of last dental appointment

1. Do you have regular dental appointments?

Yes

No

2. Have you had any trouble with previous dental treatment?

Yes No

3. Do you think saving your teeth is a waste

Yes

ino

4. Do you have any lumps or sores in your

Yes

NO

5. Have you ever had lumps or sores in your

Yes - No

6. Do you have pain in the teeth, jaws or

Yes

iNo

7. Have you ever had pain in the teeth, jaws or Yes No
head?

8. Are there any dental problems that ran in the family?

Yes

No

IV. REICIE IIISTORY:

9. Have you ever had any problems with your heart or blood vessels?

Yes

$\mathrm{No}$

10. Do you get chest pain?

11. Have you ever had rheumatic fever?

Yes

$\therefore:$

12. Do you have high blood pressure?

Yes

Nis 13. Have you ever been told you had an abnormal yes
sound in your heart?

Q Q 12

14. Have you ever had tuberculosis or emphysema? Yes

No

15. Have you ever had asthma or hay fever?

16. Do you get short of breath when lying down or when climbing stairs?

Yes No

Yes No 
17. Do you have problens it:th your bowel movements?yes No

18. Have you gained or lost weight recently? Yes No

19. Have you ever had hepatitis? Yes No

20. Have you ever had ulcers of your stomach yes No or intestines?

21. Have you ever had thyroid problems?

Yes No

22. Have you or any member of your family had diabetes?

Yes

23. Are you pregnant?

Yes

No Q己

24. Have you ever had any kidney or bladder problems?

Yes No

25. Have you ever had any trouble with your glands?

Yes

No

26. Have you ever had syphilis, gonorrhea or any other veneral disease?

Yes No

27. Have you ever passed blood in your urine?

Yes

No

28. Have you ever bled excessively following

Yes No

29. Have you ever had a blood transfusion? Why? Yes

No

30. Are you on blood thinners?

Yes

No

31. Have you ever had problems with your blood? Yes

No

32. Do you have frequent fractures or dislocations yes No

33. Do you have any joint or muscle pain?

Yes

No

34. Do you suffer from frequent or severe

Yes

No

35. Have you ever had problems with local or general anaesthesia?

Yes

iso

36. Have you ever taken medication for an

Yes

No emotional problem?

37. Have you or anyone in the family ever had fits, seizures, or convulsions?

Yes

No

38. Have you ever had a skin disease?

Yes

No

39. Do you have any eye problems?

Yes

No 
Chart \#

41. Do you have any nose problems?

Yes $\mathrm{Nb}$

42. Do you have any allergies?

43. Have you ever had any unusual reactions to medical treatment?

Yes $\quad \mathrm{Nb}$

Yes $\mathrm{Nb}$

44. Are there any diseases or medical problems that run in the family?

Yes $\mathrm{Nb}$

45. Do you smoke, drink or use non-prescription drugs?

Yes $\quad \mathrm{No}$

46. Have you ever been hospitalized?

Yes $\mathrm{Nb}$

47. Are you taking any medication?

Yes No

COMMENTS ON ANY OF THE QUESTIONS:

\section{BLOOD PRESSURE:}

DATE: 


\section{RADIOGRAPHIC STUDY PROTOCOL BWX PHASE}

GENERAL INFORMATION:

1. Enter chart number and subject name.

2. Determine which BWXs will be used for evaluation.

3. Prior to making measurements, place all BWX that will be used on the view box for simultaneous viewing.

4. Make sure lighting conditions in the room and on the view box are consistent for every evaluation.

5. At the beginning of each session, check to see that the compass is still locked on $3 \mathrm{~mm}$ (use ruler provided).

6. Always read the films from patient's Max. Right to Max.Left, then from patient's Man.Left to Mand.Right.

PART I. INDIVIDUAL SURFACE EVALUATION:

- Place the unmounted BWX in position on view box.

- Place the 1st point of the compass on the CEJ (ie., the landmark where the outer edge of the enamel of the crown intersects the outer edge of the dentin of the root). Place the 2nd compass point adjacent to the root surface at the Alveolar Crest (ie., the most coronal point of bone adjacent to the tooth surface where the periodontal ligament space has a uniform width).

- If two radiographs of the same tooth are available, the one with the more distinct landmarks is to be used.

- When in doubt, consider a surface normal.

- Enter one of the following codes for each surface on the form:

$x$ when the tooth is missing or partially erupted.

? when the surface is unreadable, whether due to overlapping image; cone-cut; CEJ or AC obscured by caries/restoration/etc; or improper film placement, exposure or processing.

+ when the 2 compass points are exceeded by the distance between the CEJ and AC.

- when the distance between the CEJ and AC does not exceed the distance between the 2 compass points.

PART II: OVERALL DIAGNOSIS:

- Based on findings from Individual surface D̂ंiagnoses:

- Circle "YES" if the ANY surface had evidence of a distance greater than $3 \mathrm{~mm}$ between the CEJ and $A C$ ie., a distance greater than the distance between the two compass points.

- Circle "NO" if the distance between the CEJ and AC is equal to or less than the distance between the two points on the compass for ALL tooth surfaces.

- If an overall diagnosis can not be made due to unreadable films, circle "UNREADABLE". 


\section{RADIOGRAPHIC STUDY PROTOCOL \\ "PA" AND "PAN" BHASE}

GENERAL INFORMATION :

1. Enter chart number.

2. Determine which radiographs will be used for evaluation

3. Place alI PAs and/or PAN that will be used in the

4. evaluation on the view box for simultaneous viewing. Make sure lighting conditions in the room and on the view box are consistent for every evaluation.

5. At the beginning of each session, check to see that the compass is still locked on $3 \mathrm{~mm}$ (use ruler provided).

6. Always read the films from patient's Max.Right to Max. Left, then from patient's Man.Left to Mand.Right.

PART I: INDIVIDUAL SURFACE EVALUATION:

. Place films in position on the view box.

- Place the 1st point of the compass on the CEJ (ie., the landmark where the outer edge of the enamel of the crown intersects the outer edge of the dentin of the root). Place the 2 nd compass point adjacent to the root surface.

- If two radiographs of the same tooth are available, the one with the more distinct landmarks is to be used.

- When in doubt, consider a surface normal.

- Based on existing PAs and/or PAN, enter one of the following codes for each surface on the form:

$x$ when the tooth is missing or partially erupted.

? when the surface is unreadable, whether due to overlapping image; cone-cut; CEJ or AC obscured by caries/restorations/etc; or improper film placement, exposure or processing.

+ when the 2 compass points are exceeded by the distance between the CEJ and $A C$.

- when the distance between the CEJ and AC does not exceed the distance between the 2 compass points. IMPORTANT NOTICE:

ON THE LINE BELOW EACH SURFACE ENTRY, INDICATE FROM WHICH

TYPE OF FILM THE SURFACE'S EVALUATION WAS MADE:

$$
\begin{array}{ll}
\text { ENTER: } & 1 \text { FOR PA } \\
& 2 \text { FOR PAN }
\end{array}
$$

PART II: OVERALL DIAGNOSIS:

- Place all PA and PAN films in position on the view box.

- Circle "YES" if the alveolar crest ( ie., the most coronal point of bone adjacent to the tooth surface where the periodontal ligament space has a uniform width) is beyond the 2nd point of the compass for ANY tooth surface.

- Circle "NO" if the distance between the CEJ and AC is equal to or less than the distance between the two points on the compass for ALL tooth surfaces.

- If an Overall Diagnosis can not be made due to unreadable films, circle, "FILM(S) UNREADABLE. 


\section{PERIODONTAL EPIDEMIOLOGY STUDY \\ BWX PHASE}

CHART NUMBER

SUBJECT NAME

\section{PART ONE: INDIVIDUAL SURFACE EVALUATION:}

Make one of the following entries for each surface:
$x$ if tooth is missing
$?$ if surface's radiographic evidence is unreadable
$+\quad$ if distance is MORE than $3 \mathrm{~mm}$ from CEJ to AC.
- if distance is $3 \mathrm{~mm}$ OR LESS from CEJ to AC.

\begin{tabular}{|c|c|c|c|c|c|}
\hline $\begin{array}{c}17 \\
\ldots M_{--}\end{array}$ & $\begin{array}{c}16 \\
\mathrm{DM}_{---}\end{array}$ & $\begin{array}{c}15 \\
\mathrm{DM}_{-}\end{array}$ & $\begin{array}{c}25 \\
\mathrm{MD}_{-}-\end{array}$ & $\begin{array}{c}26 \\
-\mathrm{MD}_{-}\end{array}$ & $\begin{array}{c}27 \\
-\mathrm{MD}_{-} \\
\end{array}$ \\
\hline$\cdots$ & $\cdots$ & $\cdots$ & $\cdots$ & $\cdots$ & $\cdots$ \\
\hline $\begin{array}{c}47 \\
D M_{---}\end{array}$ & $\begin{array}{c}46 \\
-[\mathrm{DM} \\
-\end{array}$ & $\begin{array}{c}45 \\
- \\
\end{array}$ & $\begin{array}{r}45 \\
-M D \\
\end{array}$ & $\begin{array}{c}46 \\
-M_{-}\end{array}$ & $\begin{array}{c}47 \\
- \\
\end{array}$ \\
\hline$\cdots$ & $\cdots$ & $\cdots$ & $\cdots$ & $\cdots$ & $\ldots$ \\
\hline
\end{tabular}

\section{PART TWO: OVERALL EVALUATION:}

The mesial or distal surface of ONE OR MORE 1st or 2nd molar or 2nd premolar has a distance of more than $3 \mathrm{~mm}$ between the CEJ and the alveolar crest (AC).

CIRCLE ONE:

YES

NO

BWX UNREADABLE

\section{PART THREE: (Gelskey)}

\# Surfaces with $>3 \mathrm{~mm}$ distance:

\# Present and readable surfaces:

$\%$ Present and readable surfaces with $>3 \mathrm{~mm}$ distance: 


\section{PERIODONTAL EPIDEMIOLOGY STUDY \\ "PA" AND "PAN" PHASE}

CHART NUMBER

SUBJECT NAME

I. PART ONE: INDIVIDUAL SURFACE EVALUATION:

A. Make one of the following entries for each surface:

$x$ if tooth is missing

? if surface's radiographic evidence is unreadable

$+\quad$ if distance is MORE than $3 \mathrm{~mm}$ from CEJ to $\mathrm{AC}$

- if distance is $3 \mathrm{~mm}$ OR LESS from CEJ to AC

B. Enter on lower line the TYPE OF FILM USED FOR SURFACE:

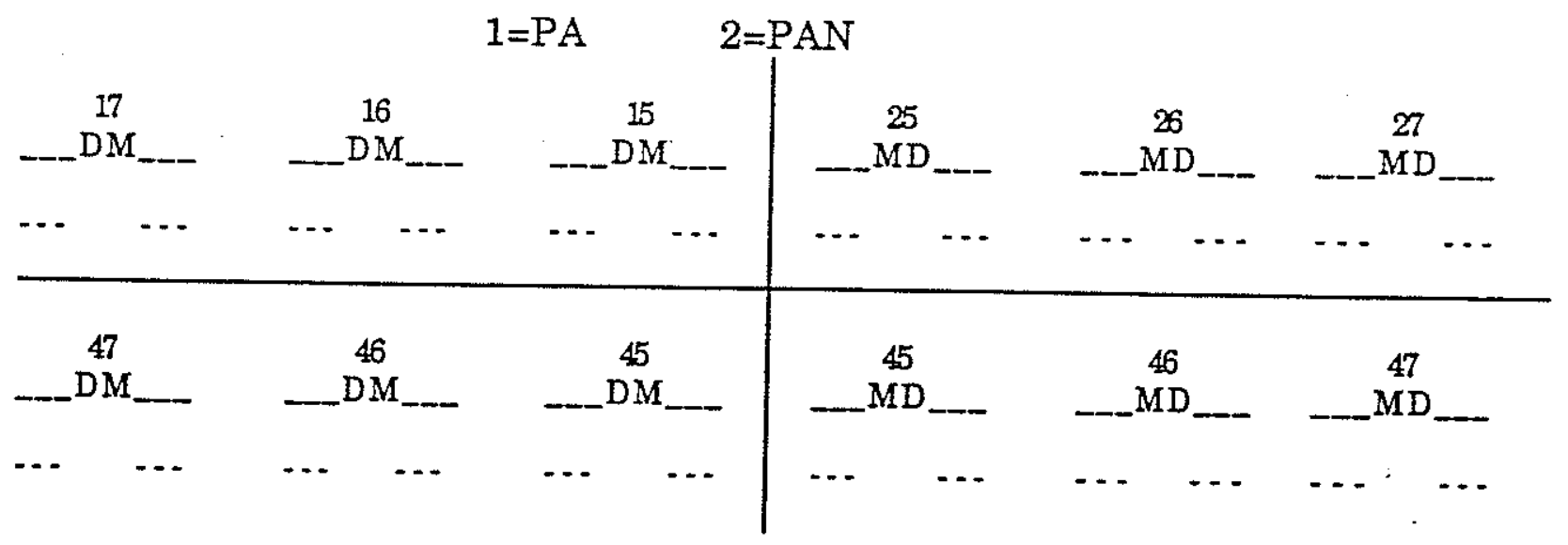

\section{PART TWO: OVERALL EVALUATION:}

The mesial or distal surface of ONE OR MORE 1st or 2nd molar or 2nd premolar has a distance of more than $3 \mathrm{~mm}$ between the CEJ and the alveolar crest (AC).

CIRCLE ONE:

YES

NO

FILM(s) UNREADABLE

\section{PART THREE: (Gelskey)}

\# Surfaces with > 3mm distance:

\# Present and readable surfaces:

$\%$ Present and readable surfaces with $>3 \mathrm{~mm}$ distance: 
Radicgraphic Equpment during past IOyears at Uof Man, FOD - from Loraine in Railiclogey

University of Manitoba, Faculty of Dentistry $00+1594$

Department of Dental Diagnostic and Surgical Sciences, Radiology Section

Equipment List

Equipment Manufacturer

- Model number

- City

- Date

PAN EQLIPLENT

General Electric - Panelipse Panoramic X-Ray System

- 46-154670G2

- Milwaukee, WI

- February 1978

S.S. White Penwalt Panorex

- 2411-220X

- Holmdel, NJ

- March 1980

PA's \& BW'S

General Electric GE-100

- 46-158800G2

- Milwaukee, WI

- February 1978

Ritter - Explorer

- J8J3 $3 \times 3146$

- Rochester, NY

S.S. White Penwalt - Intrex

- 2426-3000Y

- Holmdel, NJ

- November 1981

PROCESSING EQUIPMENT

Air Techniques Incorporated

- A/T 2000 automatic processor

- Hicksville, NY

- $43000=5 A$

Air Techniques Incorporated - PERI-PRO II X-ray

processor

- 90060

- Hicksville, NY
Recommended exposure times for all 3 techniques for posterior areas only

$8 \mathrm{~mA}, 22$ seconds, 68 to 70 $\mathrm{kVp}$

$5 \mathrm{~mA}, 20$ seconds, 68 to 70 $\mathrm{kVp}$

$15 \mathrm{~mA}, 15$ impulses, 75

$\mathrm{kVp}$

$15 \mathrm{~mA}, 1 / 5$ seconds, 80

$\mathrm{kVp}$

$10 \mathrm{~mA}, 0.4$ seconds, 70

$\mathrm{kVp}$ 
TELEPHONE INTERVIEW

Informant's Reference Number

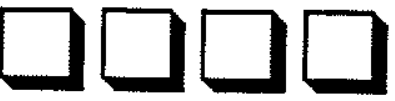

Periodontal Exam Date

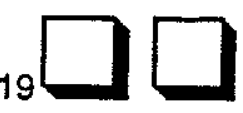

Interviewer's Name

$$
1=\text { Crawford } 2=\text { Huber } 3=\text { Gelskey }
$$
Number

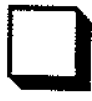

$(1,2,3)$

\begin{tabular}{|l|l|l}
\hline \multicolumn{2}{|c|}{ RECORD DATES OF CONTACT(S) AND INTERVIEW } \\
Contact Date Interview Date & Notes \\
\hline 2. & & \\
\hline Call Back Date and Time & & \\
\cline { 3 - 3 } & & \\
\hline
\end{tabular}

\section{AFTER INTERVIEW ASSESSMENT}

Circle the number which indicates your estimation of:

Low

High

Respondent's understanding of questions

Respondent's recall of information

Chance of getting same responses in two weeks

$\begin{array}{lllll}1 & 2 & 3 & 4 & 5 \\ 1 & 2 & 3 & 4 & 5 \\ 1 & 2 & 3 & 4 & 5\end{array}$

Comments:

\section{FINAL STATUS OF INTERVIEW: Check One}

1. Unable to contact. Reason(s)

2. Completed

3. a) Refused. Reason(s)

b) Obtained Level of Education:

If No, enter -88

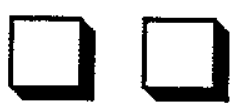

If Yes, go to Q26 and enter response

4. Other Comment(s): to $=$ $\min$ 

. You had one of your dental exams done at the Faculty that year. That was about asking several questions about TOOTH CLEANING.

Q1. Before 19 _ how often did you brush your teeth? (Do not read list)

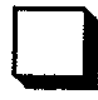

1. once or more each day

2. once or more each week

3 . once or more each month

4. rarely

5. other (specify)

6. never

-9. don't know/no response

(Prompt: Can you be more clear?) On average - how often?

Q2. Before 19_ how often did you use dental floss? (Do not read list)

1. once or more each day

2. once or more each week

3. once or more each month

4. rarely

(Prompt: Can you be more clear?)

5. other (specify)

6. never

- 9. don't know/no response

I would now like to ask you some questions about CIGARETTE SMOKING.

Q3. Before 19_, did you EVER smoke cigarettes?

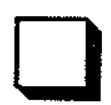

$0=$ No [Goto Q6]

$1=$ Yes

$-9=$ Don't know/no response

[Enter 1 if yes, even if it was just to "try it" and be sure to ask Q4 and Q5]

Q4. Before 19 , approximately how many cigarettes did you USUALLY smoke each day?

( 25 cigarettes per package)

Enter approximate number of cigarettes

*Enter - 88 if did not usually smoke cigarettes daily*

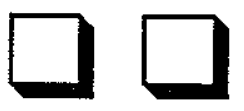

Enter - 99 if doesn't know/no response

(PROMPT: If they say they have quit, ask, "When you did smoke, approrimately how many cigarettes did you usually smoke each day?\}

$$
1 \text { pack }=25 \quad 1 / 2 \text { pack }=12 \quad 11 / 2 \text { pack }=37
$$


Q5. Altogether, approximately how many years have you smoked?

Enter approximate number of years

Enter 01 if one year or less

Enter - 99 if doesn't know/no response

(PROMPT: Try your best to estimate the number of years that you have smoked]

[Must be asked if Q3 was answered "yes"]

I would now like to ask you a few questions about BLOOD PRESSURE.

Q6. Before 19_, had you ever been told by a doctor or nurse that you had high blood pressure or hypertension?

$0=$ No. Go to Q10

$1=$ Yes

$-9=$ Can't remember/no response. Go to Q10

Q7. Approximately how old were you when you were firsi told that you had high blood pressure/hypertension? [enter age]

Enter approximate age in years

Enter -99 if can't remember/no response

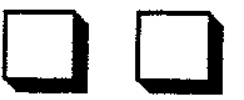

(PROMPT: Try your best to estimate your age when first told you had HBP)

Q8. Before $19 \ldots$ did you EVER take any medicine for high blood pressure or hypertension?

$0=$ No. Go to Q10

$1=$ Yes

$-9=$ Can't remember/no response. Go to Q10

Q9. Altogether, approximately how many years have you taken medication(s) for hypertension/high blood pressure?

Enter approximate number of years medication taken Enter 01 if one year or less

Enter - 99 if doesn't know/no response 
Now, I would like to ask you a few questions about HEART DISEASE.

Q10. Before 19_, did your doctor ever tell you that you had any of the following conditions? I will read a list. Please say yes or no to each condition. (Read List)

$$
\begin{array}{ll}
\text { Enter } 0=\text { No } & \text { If yes, ask “Approximately how } \\
\text { Enter } 1=\text { Yes } & \text { old were you when you were first } \\
& \text { told? } \\
& \text { [Enter age in years] } \\
& \text { [Enter }-99 \text { if doesn't know/ no } \\
& \text { response or not applicable] }
\end{array}
$$

1. Heart attack

1.
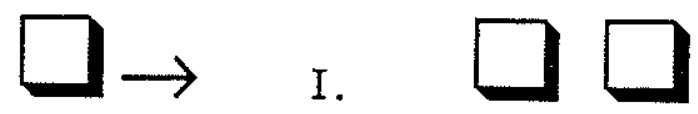

Prompt: Sharp pain, usually hospitalized

2. Angina

2.

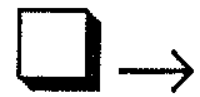

II.

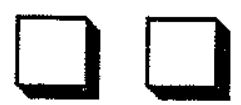

Prompt: Sharp chest pain $=$ Nitroglycerine

3. Hardening of Arteries

3.

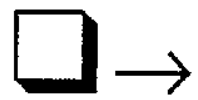

III.

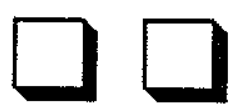

Prompt: Problems with blood vessels (Athero/Arteriolosclerosis)

$$
\text { If all three = "No", go to Q13 }
$$

Q11. Before 19_, did a doctor prescribe medication for [Read condition(s) they reported in Q10]?

Enter 0,1 or -9 for each box

$0=$ no, $1=$ yes, $-9=$ not relevant

1. Heart Attack Medications

[Prompt: eg. Inderol, Nitroglycerine, Aspirin]

2. Angina Medications

[Prompt: eg. Nitroglycerine]

3. Hardening of Arteries

[Prompt: eg. Cholesterol lowering meds, Aspirin]

$$
\text { If all three }=0 \text { or }-9 \text {, go to Q13 }
$$

Q12. Altogether, approximately how many years did you take this/these medicines? [Enter number of years or -99 if can't remember/no response]

1. For heart attack

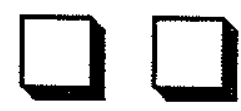

2. For Angina

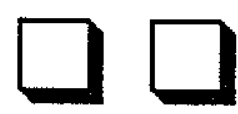

3. For Hardening of Arteries

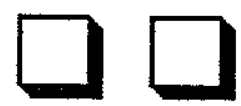

[Ask them to get medication bottle(s) if unclear] 
The next few questions are about DLABETES.

Q13. Before 19_, had you ever been told by a doctor that you had diabetes?

$0=$ No. Go to Q17

$1=$ Yes

$2=$ Borderline

$-9=$ Can't remember/no response. Go to Q17

Q14. Approximately how old were you when you were first told that you had

Enter approximate age in years

Enter -99 if not sure/don't remember

(Prompt: Try your best to give approximate age.)

Q15. Before 19___ were you ever on any treatment for your diabetes?

$0=$ No. Go to Q17

$1=$ Yes

$-9=$ Can't remember/no response. Go to Q17

Q16. What treatment(s) have you EVER been on for diabetes? I will read a list. Please say yes or no to indicate whether you have EVER used the following
treatments

$$
\begin{gathered}
\text { Enter } 0=\text { No } \\
\text { Enter } 1=\text { Yes } \\
\text { Enter } \cdot 9=\text { Can't remember/don't know }
\end{gathered}
$$
A. Insulin
B. Pills to control blood sugar
C. Diet/weight loss
D. Any other treatment? (Specify) 


\section{The final medical questions are about OTHER MEDICINES.} Q17. Before 19
medicines?

$$
\begin{aligned}
& \text { Enter 0 = No } \\
& \text { Enter 1 = Yes }
\end{aligned}
$$

1. Dilantin/Phenytoin

(Prompt: For epilepsy or seizures)

2. Cyclosporin/Sandimmune

(Prompt: To prevent transplant rejection)

3. Nifedipine/Adalat
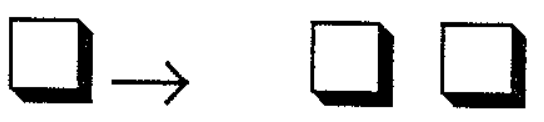

Approximately how many years did you take medication(s)
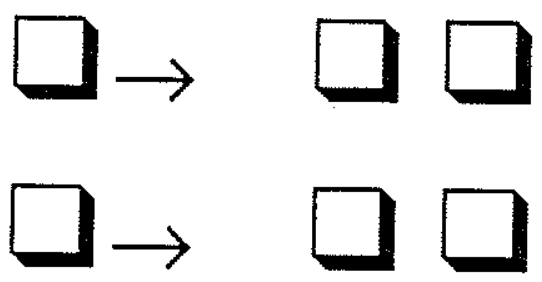

(Prompt: For angina, hypertension)

[*Ask Women Only.] [ENTER -8 AND -88 FOR MEN]

4. Birth Control Pills, or Estrogen/
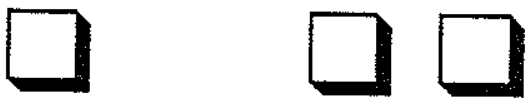

Progestrone/Provera

(Prompt: Hormone replacement therapy - post menopause - post hysterectomy)

For Women: Enter actual number of years each was taken

Enter 01 if one year or less

Enter - 99 if doesn't know/no response

Enter - 99 if YEARS ARE NOT APPLICABLE

Q18a. Before 19 did you regularly take ANY OTHER pills or medicines MORE THAN THREE TIMES EACH WEEK?

$0=$ No Go to Q19

$1=$ Yes GotoQ18b

$-9=$ Can't remember/no response. Go to Q19

Q18b. Did you take ANY of the following anti-inflammatory or pain medicines MORE THAN three times each week? [Read list slowly]

Aspirin (ASA), Bufferin, Entrophen, Advil, Motrin, Volteran, Indocid, Dolobid, Toradol, Anaprox or any other pills for Arthritis or Heart: (specify)

$0=N_{0}$

$1=$ Yes

$-9=$ Can't remember 
The last few questions let us look at HEALTH FACTORS BY DIFFERENT GROUPS LIKE AGE, GENDER, OCCUPATION, ETC.

Q19. On a scale of 1 to 4, "one" being not stressful and "four" being extremely stressful, how stressful was your life before 19 adult have you had ... [Read List slowly] ? In other words, as an

1. no stress

2. slight stress

3. moderate stress

4. extreme stress

-9 Don't know/no response

[Prompt: Try to describe your overall average level of stress]

[Prompt: some examples of life stress are $=$ death in family, divorce, war, job loss, serious illness, etc.]

Q20. What is the postal code of your current home?

Enter all -9's for don't know/no responses

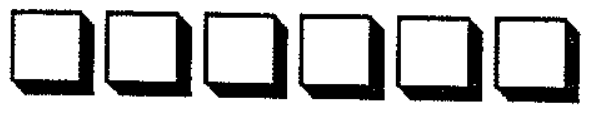

$$
\text { [Repeat code - and say "V - as in Victor", etc.] }
$$

Q21. Enter respondent's sex

$$
\begin{array}{rll}
0 & = & \text { Female } \\
1 & = & \text { Male } \\
-9 & = & \text { No response }
\end{array}
$$

Q22. What is your date of birth?

Enter -99 if don't know/no response

$\begin{array}{lll}\text { Jan 01 } & \text { May 05 } & \text { Sept 09 } \\ \text { Feb 02 } & \text { June 06 } & \text { Oct 10 } \\ \text { Mar 03 } & \text { July 07 } & \text { Nov 11 } \\ \text { Apr 04 } & \text { Aug 08 } & \text { Dec 12 }\end{array}$

Month

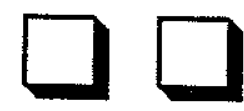

Day

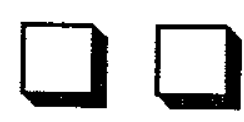

Year 19 
Q23. What is your current employment status. I will read list. Please stop me when I describe your status? (Read this list)

1. full-time (i.e. 35 hours or more a week) $] \longrightarrow \rightarrow$ Go to Q23a

3. unemployed

4. laid off

5. retired

6. other (specify)

7. homemaker

8. student

-9. don't know/no response

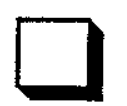

Q23a. Do you work more than one job to make up these hours?

$$
\begin{array}{lll}
0 & = & \text { No } \\
1 & = & \text { Yes }
\end{array}
$$

Q24. What is/was your occupation?

[Enter their exact job as they describe it.

Enter their primary or main job if they had more than one.]

(Prompt: What is/was your usual job?)

Q25. What is your marital status? I will read a list. Are you ... [Read list]

1. Single (by that I mean never married)?

2. Married (and not separated) or Living Common Law?

3. Divorced or Separated?

4. Widowed or Widower?

-9. Don't know/no response 
Q26. What is the HIGHEST grade or level of education you have ever ATTENDED or completed [mark only one]

\section{ENTER:}

$01 \quad$ No schooling

$\begin{array}{ll}02 & \text { Some } \\ 03 & \text { Completed }\end{array}$

$\begin{array}{ll}04 & \text { Some } \\ 05 & \text { Completed }\end{array}$

06 Some

07 Completed

Elementary [K-9]

08 Some

09 Completed

Secondary [10-12]

10 Some

11 Completed

\} Community College, Technical College non-degree certificate or diploma

12 Other (specify)

-99 Doesn't know/no response

227. For statistical purposes only, we need to know the range of your total, gross family household income. Could you please indicate from the following list, the income range for your household last year?

\section{Read List}

1. under $\$ 12,000$

2. $\$ 12,000$ to $\$ 24,999$

3. $\$ 25,000$ to $\$ 49,999$

4. $\$ 50,000$ to $\$ 74,999$

5. $\$ 75,000$ and over

*6. Respondent refused to answer question

-9. Don't know 
Q28. Would you like to receive a summary of this study's finding?

$$
\begin{array}{ll}
0 & =\quad \text { No } \\
1 & =\text { Yes }
\end{array}
$$

If "yes", ask, "What is your current address?"

Enter:

$$
\text { Box/Street }
$$

$$
\overline{\text { City }}
$$

Province

$$
\text { Postal Code }
$$

Thank you for taking time to answer these questions. We appreciate your cooperation.

\section{N.B. INTERVIEWER:}

Be sure to complete the three sections on Page 1 of Interview immediately following the interview.

1. Record Dates of Contact(s) and Interview

2. After Interview Assessment

3. Final Status of Interview 


\section{COMMITEEON HUMAN SURUECTS INVOLVED INRESEARCH}

Name of investigator

Professor. S. C. Gelskey

Yeur proiect entitled

Risk Indicators for Pericdontitis among a Dental Teaching-Clinic Population

As revised on No revisions required.

Has been approved by the committee.

\section{EEASENOTE}

Any significant changes in the approved protocol must be reported to the Chair of the committee for the Committee's consideration, prior to the implementation of the changes in the protocol.
Committee Reference. EC $18 / 948$

Date. 24 February 1994 
- May I please speak with $?$

- Hello, this is Clinic, at the Faculty of Dentistry, University of Manitoba. calling from the Dental

- Did you receive the letter of information that we sent you?

[If no, ask them for their current address so that another copy can be sent. Tell them that you will call back in 1-2 weeks.]

- As you know, in cooperation with the Faculty of Dentistry, Prof. Gelskey is conducting a study of the patients who have recently received care at the Dental College Clinic. Patients selected for the study will be interviewed and a portion of their dental chart copied for the study. This study is part of Prof. Gelskey's PhD Thesis research.

We would appreciate if you would be willing to answer some questions about your dental and general health and about yourself. This telephone interview will take about 20 minutes and all information you share with me will remain confidential and anonymous.

You are not likely to benefit directly from participating in this study; however, we do hope that we can learn from this study so that the Faculty can continue to serve the community effectively.

Would you be willing to answer a few questions for me at this time?

(If they say "YES", begin the interview with Q1).

(If they say "NO":

I. Ask: Would you be willing to participate in this survey at a more convenient time? When: enter call back time on their interview form.

IIA. If they say "No" ask: May I ask why you are not willing to participate? Enter reason on their interview form.

IIB. Would you be willing to answer just one question about your education?

If No, enter - 88

If Yes, ask Q26 only. Enter Q26 response.

Thank them for their cooperation. 